Université de Montréal

\title{
Doppler vortography
}

\section{Detection and quantification of the vortices in the left ventricle}

\author{
par \\ Forough Mehregan \\ Institut de génie biomédical \\ Faculté de médicine \\ en génie biomédical
}

Mémoire présenté à la Faculté des études supérieures en vue de l'obtention du grade de maîtrise ès sciences appliquées (M.Sc.A.)

Septembre 2013 
(C) Forough Mehregan, 2013

Université de Montréal

Faculté des études supérieures et postdoctorales

Ce mémoire intitulé:

\title{
Doppler vortography
}

\section{Detection and quantification of the vortices in the left ventricle}

\author{
Présenté par: \\ Forough Mehregan
}

a été évalué par un jury composé des personnes suivantes:

M. Philippe Comtois, $\mathrm{Ph}$. D, président rapporteur

M. Damien Garcia, Ph. D, directeur de recherche

M. Guy Cloutier, Ph. D, codirecteur de recherche

M. Nicolas Noiseux, M.D., membre du jury 


\section{RÉSUMÉ}

Nous proposons une nouvelle méthode pour quantifier la vorticité intracardiaque (vortographie Doppler), basée sur l'imagerie Doppler conventionnelle. Afin de caractériser les vortex, nous utilisons un indice dénommé «Blood Vortex Signature (BVS) » (Signature Tourbillonnaire Sanguine) obtenu par l'application d'un filtre par noyau basé sur la covariance. La validation de l'indice BVS mesuré par vortographie Doppler a été réalisée à partir de champs Doppler issus de simulations et d'expériences in vitro. Des résultats préliminaires obtenus chez des sujets sains et des patients atteints de complications cardiaques sont également présentés dans ce mémoire. Des corrélations significatives ont été observées entre la vorticité estimée par vortographie Doppler et la méthode de référence (in silico: $\mathrm{r}^{2}=0.98$, in vitro: $\mathrm{r}^{2}=0.86$ ). Nos résultats suggèrent que la vortographie Doppler est une technique d'échographie cardiaque prometteuse pour quantifier les vortex intracardiaques. Cet outil d'évaluation pourrait être aisément appliqué en routine clinique pour détecter la présence d'une insuffisance ventriculaire et évaluer la fonction diastolique par échocardiographie Doppler.

Mots-clés : Echocardiographie Doppler, Écoulement sanguin intraventriculaire, Imagerie de vortex, Vorticité, Vortographie Doppler 


\begin{abstract}
We propose a new method for quantification of intra-cardiac vorticity (Doppler vortography) based on conventional Doppler images. To characterize the vortices, an index called "blood vortex signature" (BVS) was obtained using a specific covariance-based kernel filter. The reliability of BVS measured by Doppler vortography was assessed in mock Doppler fields issued from simulations and in vitro experimentations. Some preliminary results issued from healthy subjects and patients with heart disease were also presented in this research project. Strong correlations were obtained between the Doppler vortography-derived and groundtruth vorticities (in silico: $\mathrm{r}^{2}=0.98$, in vitro: $\mathrm{r}^{2}=0.86$, in vivo: $\mathrm{p}=0.004$ ). Our results demonstrated that Doppler vortography is a potentially promising echocardiographic tool for quantification of intra-ventricular vortex flow. This technique can be easily implemented for routine checks to recognize ventricular insufficiency and abnormal blood patterns at early stages of heart failure to decrease the morbidity of cardiac disease.
\end{abstract}

Keywords: Doppler echocardiography, Intra-ventricular blood flow, Vortex imaging, Vorticity, Doppler vortography 


\section{TABLE OF CONTENTS}

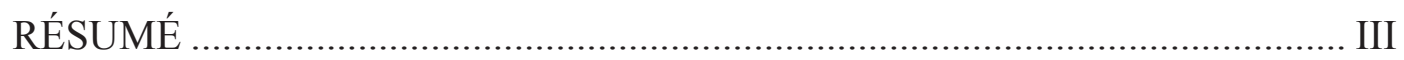

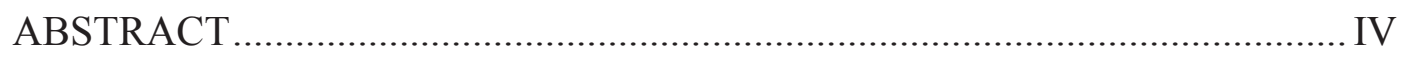

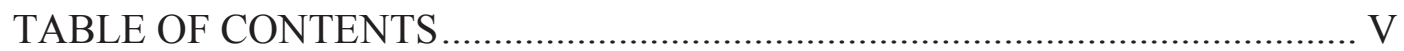

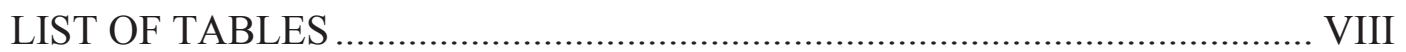

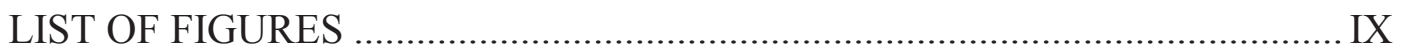

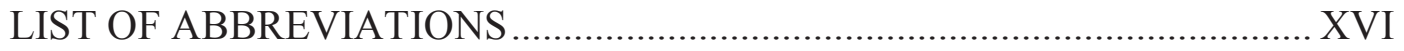

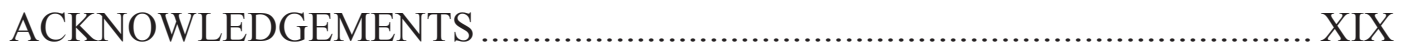

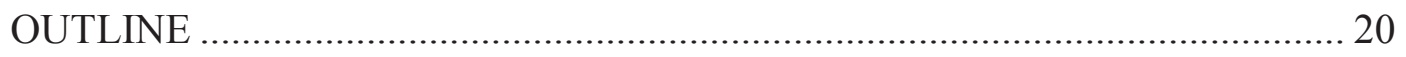

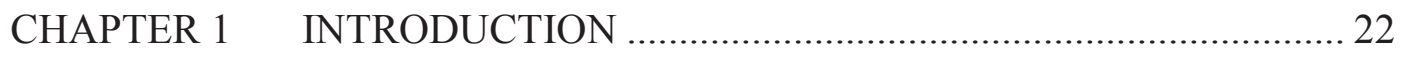

$1.1 \quad$ Heart and left ventricle.................................................................... 22

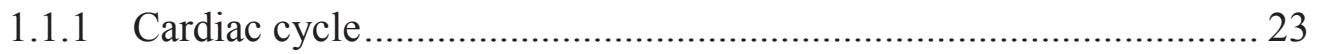

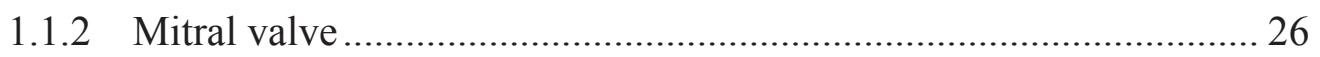

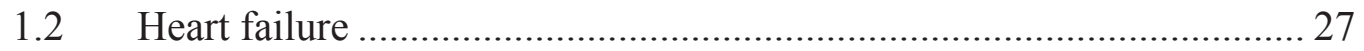

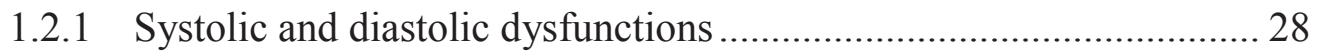

1.3 Current diagnostic tools for left ventricle dysfunction ……….............. 33

1.3.1 The electrocardiogram (ECG) tracing................................................ 33

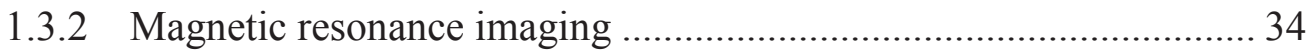

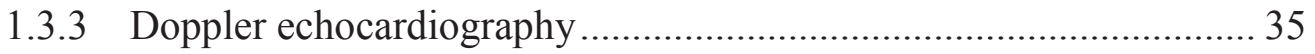

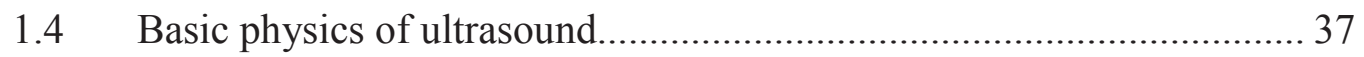

1.4.1 Propagation of ultrasound waves ....................................................... 37

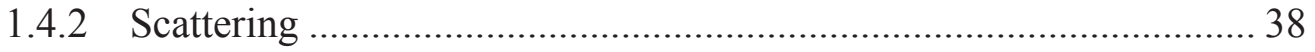

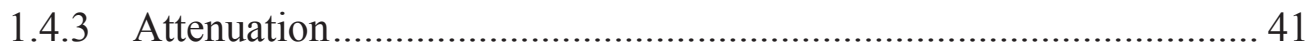




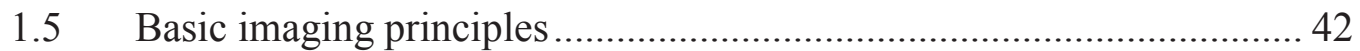

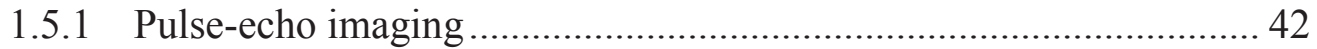

1.5.2 Transducer........................................................................... 42

1.6 Echo display modes ............................................................... 46

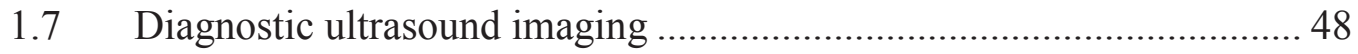

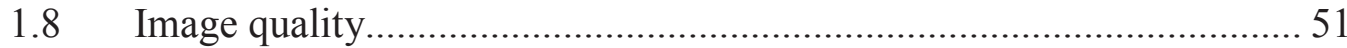

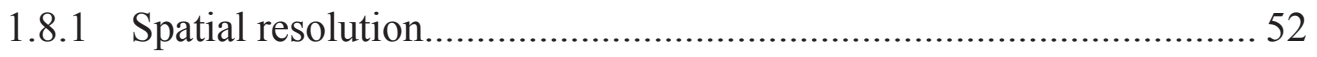

1.8.2 Contrast resolution and signal-to-noise ratio ............................. 52

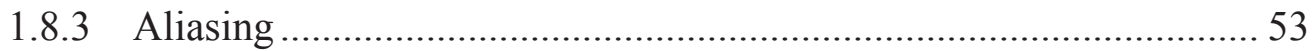

CHAPTER 2 BACKGROUND AND OBJECTIVES ................................... 54

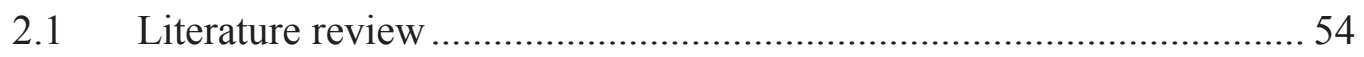

2.1.1 Vortex and vorticity definition.............................................. 54

2.1.2 Vortex formation during diastolic filling ..................................... 56

2.1.3 Effect of left ventricle diseases on vortex formation ...................... 59

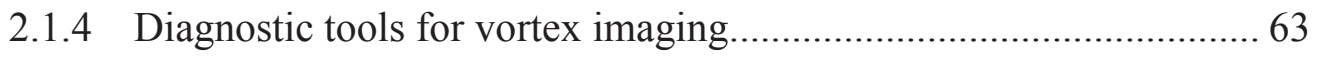

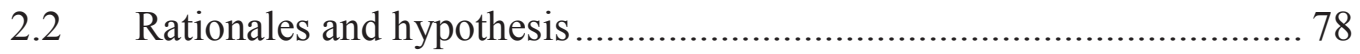

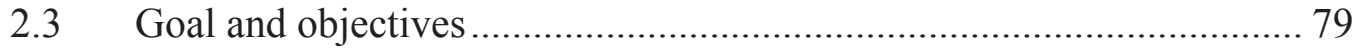

CHAPTER 3 MATERIALS AND METHODS ......................................... 80

3.1.1 In silico: ultrasound imaging and simulations ............................. 88

3.1.2 In vitro analysis based on PIV experimentation in a left heart model91

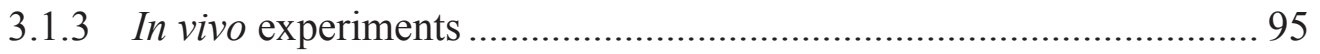

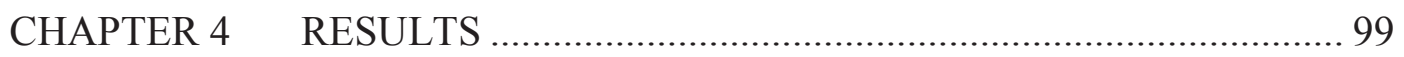

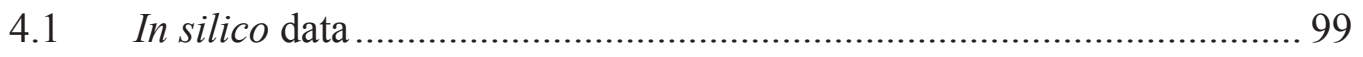

4.1.1 Doppler-derived vs. Ground-truth vorticity in silico ........................ 99 
4.1.2 Effect of kernel size in silico ..................................................... 103

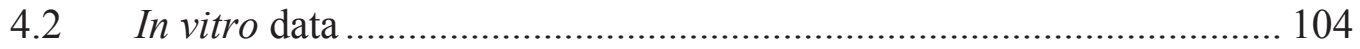

4.2.1 Vortography-derived vs. ground-truth vorticity.......................... 104

4.2.2 Effect of kernel size and angle insonification ............................ 107

4.3 In vivo study: Doppler vortography ............................................. 109

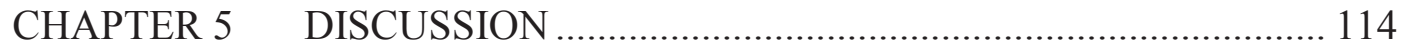

5.1 Presence of mitral and apical vortices.......................................... 114

5.2 Why using two-dimensional assumption? .................................... 120

5.3 Technical limitations of the study ............................................ 121

$5.4 \quad$ Future perspectives ............................................................. 122

5.4.1 Future validations............................................................. 122

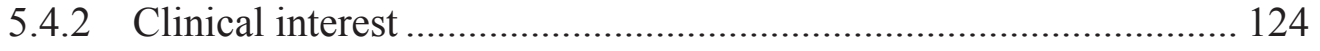

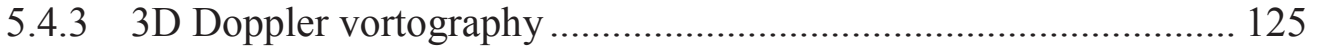

5.4.4 Develop a user-friendly software .......................................... 126

CHAPTER 6 CONCLUSION ............................................................. 127

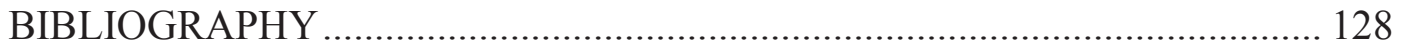

APPENDIX 1 - PROCEEDING ARTICLE [71] ........................................XX

APPENDIX 2 - JOURNAL ARTICLE [144] ............................................XXI 


\section{LIST OF TABLES}

Table 4.1: Pairwise comparison among 5 different kernel sizes in silico. The mean vorticity differences with standard errors are summarized. 103

Table 4.2: Pairwise comparisons using repeated measures ANOVA within 5 selected kernel sizes in vitro. The mean vorticity differences with standard errors are represented. 108

Table 4.3: The results of the pairwise comparisons using repeated measures ANOVA within 5 different insonification angles are represented. The mean vorticity differences with standard errors and P-value are given. 109 


\section{LIST OF FIGURES}

Figure 1-1: Sequence of blood circulation in a normal heart [3].

Figure 1-2: Events of the cardiac cycle involving the left heart [5]. Different phases of cardiac cycle are as follows: 1) atrial contraction, 2) isovolumetric contraction, 3) rapid ejection, 4) reduced ejection, 5) isovolumetric relaxation, 6) rapid filling, and 7) reduced filling. LV, left ventricle; ECG, electrocardiogram; AP, aortic pressure; LVP, left ventricle pressure; LAP, left atrial pressure; LVEDV, left ventricle end diastolic volume; LVESV, left ventricle end systolic volume. 25

Figure 1-3: Side view (left) and top view (right) of the mitral valve [6]. 26

Figure 1-4: The mitral valve function during diastole (left) and systole (right) [7].

Figure 1-5: LV pressure volume loop which represents ventricular pressure against ventricular volume at any given point in a single cardiac cycle. Different period of a heart cycle is represented by letters. a, ventricular filling; b, isovolumetric contraction; c, ventricular ejection; $d$, isovolumetric relaxation. ESV, end systolic volume; EDV, end diastolic volume; SV, stroke volume; EDPVR, end diastolic pressure volume relationship; ESPVR, end systolic pressure volume relationship [5].... 30

Figure 1-6: Ventricular passive filling curve (EDPVR) which provides an insight into the ventricular diastolic function. The EDPVR for normal, hypertrophic, and dilated left ventricles are represented. The slope of the EDPVR increases in hypertrophic patients, however it decreases in the presence of ventricular dilation. LV, left ventricle; EDV, end diastolic volume; EDP, end diastolic pressure [5].

Figure 1-7: The components of a normal electrocardiogram (ECG). P: atrial depolarization; QRS: ventricular depolarization; T: ventricular repolarization. 
Figure 1-8: Propagation of longitudinal waves, consider the rarefaction and

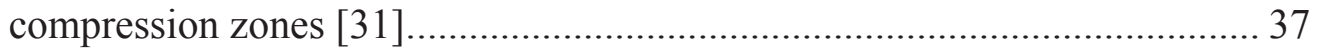

Figure 1-9: Reflection and refraction of ultrasound occurs at the tissue boundaries. With perpendicular incident to a boundary, a portion of the beam is transmitted and a portion of it is reflected back to the probe $[29,32]$.......... 39

Figure 1-10: Illustration of the scattering of the ultrasound wave in contact with a small particle or rough surface $[29,32]$.

Figure 1-11: Linear and curvilinear ultrasound transducers [29]. 43

Figure 1-12: Implementation of delay to control beam focusing and steering [34].

Figure 1-13: Illustration of a linear array and phased array transducer [30]. 45

Figure 1-14: B-mode scanning principle based on the displaying the echoes as bright dots in a time-base line [35]. 46

Figure 1-15: An example of a B-mode scanning of a normal heart in an apical 3chamber view [36]. LV, left ventricle; RV, right ventricle; LA, left atrium; $\mathrm{MV}$, mitral valve; AO, aorta; $\mathrm{AOV}$, aortic valve. 47

Figure 1-16: Color flow Doppler imaging in an apical 4-chamber view of a normal heart during diastole [37]. LV, left ventricle; RV, right ventricle; LA, left atrium; RA, right atrium. 47

Figure 1-17: Diagnostic imaging sequence in a typical conventional ultrasound system [28]. 50

Figure 2-1: The boundary layer separation leading to the vortex formation. Dark and light colors represent the clockwise and counter-clockwise vorticities [42]. 55

Figure 2-2: Blood flow directions, streamlines, and circulating regions (vortices) in a healthy left ventricle during early (left) and late (right) diastole acquired by Echo-PIV are shown. The kinetic energy of the streamlines is represented by 
colors. The large anterior and smaller posterior mitral vortices are present on sides of the mitral inflow jet during diastole [49]. 57

Figure 2-3: The blood velocity mapping of a normal LV during diastole and systole overlaid on the corresponding anatomical B-mode image [47]......... 58

Figure 2-4: 2D color Doppler blood velocity mapping of 3 different left ventricles (normal, dilated and severely dilated subjects) in apical long-axis view. The arrows show the direction of the vortices. Black lines are streamlines and the color shades represent the magnitude of the velocity as shown in the color bar [47].

Figure 2-5: Vortex formation in the left ventricle. An apical vortex appears in patients with diastolic dysfunction. MV, mitral valve; $\mathrm{AV}$, aortic valve. ..... 63

Figure 2-6: Phase contrast MRI at the time of early diastole inflow in a normal heart. In a 2D plane, blood moves forward into the left ventricle and when it reaches the apex it makes a smooth turn into the outflow tract [93].

Figure 2-7: Blood flow distribution in carotid bifurcation acquired by Doppler with a fixed probe position in three different beam angles $\left(\alpha=70^{\circ}, 90^{\circ}, 110^{\circ}\right)$ [101].

Figure 2-8: Reconstructed flow field in the carotid bifurcation [101] 69

Figure 2-9: Schematic of echo-PIV technique. Contrast agents are seeded into the flow as tracers. Displacement of the particles position and also the velocity vectors can be determined by analyzing two consecutive B-mode images with PIV algorithm. The B-mode images are acquired by ultrasound imaging system [111]. 71

Figure 2-10: An example of vector flow mapping. The flow velocity vectors are superimposed on the color Doppler images. The velocity field at a single point is represented by red dots and the yellow arrows [49]. AV, aortic valve; LA, left atrium; LV, left ventricle. 74 
Figure 2-11: Flowchart of Garcia is method for reconstruction of the velocities perpendicular to the scan-line [63].... 76

Figure 2-12: Examples of blood velocity mapping on 3 volunteers (normal, hypertrophied, and dilated subjects) in apical long-axis view overlaid on Bmode images. The color shades represents the magnitude of velocities as shown in color bar. The arrows indicate the flow direction [63]. 77

Figure 3-1: A) Single large vortex (Thick arrowed streamlines) and corresponding Doppler field; the phased array transducers at the top. B) Corresponding blood vortex signature (BVS), the BVS amplitude is maximal at the center of the vortex 80

Figure 3-2: Left: a numerical vortex (vortex pair) is scanned by a probe. Right: corresponding Doppler data. At the vortex center the velocity is nearly zero surrounded by two maxima of opposite signs. 81

Figure 3-3: Basic concepts of blood vortex signature (BVS). R and S stand for the ramp and sign functions, respectively. Left panel: raw Doppler data of a vortex pair in the Cartesian coordinate system.

Figure 3-4: Flowchart of Doppler vortography method for specifying the main vortices and calculating their core vorticities (vortography-derived vorticities) in the left ventricle during diastole phase. 85

Figure 3-5: A) Numerical model: a vortex pair is scanned with a cardiac probe. B) Corresponding Doppler velocities (without additive noise). C) Vorticity map (reference) calculated from the original vector flow field. D) BVS map related to the Doppler field in B 86

Figure 3-6: The rectangular contour used to calculate circulation and estimate the vorticity at point $(\mathrm{i}, \mathrm{j})[122]$. 87

Figure 3-7: Flowchart of in silico simulation using Field II software. The scatterers were displaced according to the Lamb-Oseen vortex $(V x, V z)$ which was performed between each image acquisition. The time between each image 
acquisition (TPRF) was adjusted so that aliasing was avoided. (X1, Z1) and $(\mathrm{X} 2, \mathrm{Z2})$ are position of the point scatterers before and after displacement respectively.

Figure 3-8: Left panel: overview of the in vitro setup [130]. The different components are (1) laser, (2) micrometric displacement system, (3) camera, (4) pulmonary veins, (5) left atrial, (6) left ventricle, and (7) systemic model. Right panel: zoom the left atrio-ventricular device (parts (5) and (6)) [129]. 92

Figure 3-9: Flowchart of the in vitro experiment. The vortography-derived vorticity of the main diastolic vortex was compared with the ground-truth vorticity calculated by 8 point circulation method.

Figure 3-10: Effects of transducer position on the reconstructed Doppler velocity field in the in vitro experiment were analyzed. Several insonification angles $(0 \mathrm{o}, \pm 5 \mathrm{o}, \pm 10 \mathrm{o})$ were tested.

Figure 3-11: Patient in the left lateral recumbent position prepared for the echocardiographic examination (GE Healthcare, Vivid E9). 97

Figure 3-12: The graphical user interface for fast and easy detection and quantification of the intra-ventricular vortices by Doppler vortography. This interface is able to read and post-process the raw Doppler data issued from a GE ultrasound scanner. In this example the yellow circle represents the mitral vortex whose vorticity is $258 \mathrm{~s}^{-1}$

Figure 4-1: In silico studies. Color Doppler fields were simulated for Lamb-Oseen vortices using Field II (see left panel). The blood vortex signature was deduced from the color Doppler data using Eq. 3-2 and allowed detecting the vortex core (see right panel). The core vorticity yielded by Doppler vortography (Eq. 3-5) was compared with the theoretical core vorticity (Eq. 3-11). 100 
Figure 4-2: Concordance between the vortography-derived and ground-truth vorticities in silico for different kernel sizes using linear regression. 101

Figure 4-3: Relative errors in silico calculated for different kernel sizes using the Bland-Altman plot. 102

Figure 4-4: In vitro data. Color Doppler fields were created from in vitro intraventricular PIV (particle image velocimetry) velocity fields. The blood vortex signature was measured by Doppler vortography and the vorticity of the main diastolic vortex was compared with the PIV-derived vorticity..... 105

Figure 4-5: In vitro results. Comparison between vortography-derived (Eq. 3-5) and ground-truth (Eq. 3-8 and 3.9) vorticities. Several angle insonifications (see fig. 3.10) were simulated for specified kernel window sizes. 106

Figure 4-6: Relative errors in vitro calculated for different Kernel sizes using the Bland-Altman plot. 107

Figure 4-7: Vortex detection by Doppler vortography. Vortices are denoted by circles whose radii are related to the vorticity. 110

Figure 4-8: Raw Doppler data (left panel) and corresponding BVS maps (right panel) of three subjects (A. healthy, B and C. patients) during early filling. 111

Figure 4-9: Performance of the "apical vortex" test in normal and hypertensive (HTN) patients.

Figure 4-10: Left: Median of mitral core vorticities over 3 to 10 cycles for normal and hypertensive (HTN) groups. Right: Comparison of the mitral core vorticities between the normal and hypertensive groups.

Figure 5-1: Doppler vortography in clinical practice. The BVS maps of three subjects (one normal and two patients) are presented. Only one mitral vortex at the vicinity of the anterior mitral valve is present in healthy subject. An abnormal apical vortex may also appear in patients with diastolic impairment. 
Figure 5-2: Raw Doppler data (left panel) and corresponding BVS patches (right panel) of two patients during early filling. An extra counter-rotating apical vortex was visible in most of the patients during diastole (A). In some patients a large vortex was present adjacent to the posterior mitral valve

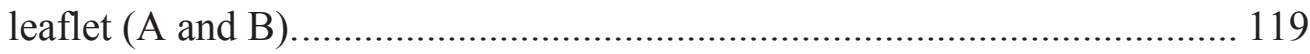




\section{LIST OF ABBREVIATIONS}

1D One dimensional

2D Two dimensional

3D Three dimensional

AO Aorta

AOV Aortic valve

AP $\quad$ Aortic pressure

BVS Blood vortex signature

DCM Dilated cardiomyopathy

ECG Electrocardiogram

EDP End diastolic pressure

EDPVR End diastolic pressure volume relationship

EDV End diastolic volume

EF Ejection fraction

ESV End systolic volume

HCM Hypertrophic cardiomyopathy

HF Heart failure

HTN Hypertensive

IQ In-phase quadrature

IVR Isovolumetric ventricular relaxation

LA Left atrium

LAP Left atrium pressure

LV Left ventricle 


\begin{tabular}{ll} 
LVEDV & Left ventricle end diastolic volume \\
LVESV & Left ventricle end systolic volume \\
LVP & Left ventricle pressure \\
MRI & Magnetic resonance imaging \\
MV & Mitral valve \\
PCMRI & Phase contrast magnetic resonance imaging \\
PCWP & Pulmonary capillary wedge pressure \\
PIV & Particle image velocimetry \\
PRF & Pulse repetition frequency \\
PSF & Point spread function \\
PV & Pressure-volume \\
RF & Radio frequency \\
RV & Right ventricle \\
SNR & Signal-to-noise \\
TFC & Stroke volume \\
VFM & Vime gain compensation \\
\hline &
\end{tabular}


xviii

Dedicated to my supportive parents,

Mahnaz and Naser, and my soulmate Ehsan. 


\section{ACKNOWLEDGEMENTS}

First, I would like to express my sincere appreciation to my advisor Dr. Damien Garcia for his continual support during my M.Sc. research study, for his excellent guidance, patience, motivation and, of course, immense knowledge in cardiovascular biomechanics. Without his supervision and constant help this study would not have been possible. In addition, a thank you to Dr. Guy Cloutier, for his seasoned suggestions which kept me focused on this research topic.

Furthermore, I would like to thank my master defense committee, Dr. Philippe Comtois and Dr. Nicolas Noiseux, for their encouragement, insightful comments and hard questions.

I would also like to acknowledge the Canadian Institutes of Health Research (CIHR) and the Heart and Stroke Foundation of Québec (HSFQ) for the financial support granted to complete this research project.

Thank you to my fellow lab-mates in the RUBIC and LBUM research groups for the stimulating discussions and for providing me with an excellent atmosphere for doing research. In particular, I am grateful to Stephan Muth, who was always willing to help me with programming in Matlab and for giving his best suggestions.

Most of all I would like to thank my husband, Ehsan Maghsoudi, who was always there to cheer me up and stood by me through the challenges I encountered while writing this dissertation.

Last, but of course not least, I want to thank my parents, Mahnaz Ghanevati and Naser Mehregan, for their sacrifice and unconditional love. Words can not express the appreciation I feel for my parents whose dedication, and persistent confidence in me, has taken the load off my shoulder. They were always beside me not only over the course of my academia, but also during happy and hard moments throughout my life. 


\section{OUTLINE}

Heart disease is a leading cause of death worldwide and most patients with heart disease suffer from symptoms of impaired left ventricle myocardial function. Several studies have aimed to predict and evaluate LV function to decrease its effect on cardiac morbidity and mortality. The objective of this research project was to create a new non-invasive clinical index based on vortical structures for the assessment of left ventricle function that can be easily applicable in a clinical context. For this purpose, we start this thesis with an overview of heart, left ventricle, and mitral valve physiology. Then heart failure epidemiology along with systolic and diastolic dysfunctions and the reliable parameters to evaluate diastolic function will be discussed. Current diagnostic tools for left ventricle dysfunction will be briefly investigated. Conventional color Doppler ultrasound imaging is a fully non-invasive, available and inexpensive modality to detect and quantify the intra-ventricular vortices that form during left ventricle filling. Therefore, in this section the physics of the ultrasound wave propagation and more specifically, the scattering and reflection processes in the medium which produce the backscattered signal received by the transducer will be explained. Transducer design, ultrasound beam formation and the process of beam steering and focusing in an ultrasound image acquisition as well as the different types of echo display modes will subsequently be discussed. Finally, the diagnostic imaging sequence in a typical conventional ultrasound system and the influence of different parameters on image quality will be investigated.

Within the left ventricle of a normal heart, blood flows in an asymmetric swirling motion. These swirling patterns (also called vortices) are claimed to minimize the energy dissipation from the flow and optimize ejection of the blood flow from the left ventricle [1,2]. Vortices that form during left ventricle filling have specific geometry and location which can be a determinant factor of the heart function. So, in chapter 2, the origin of the vortices and their strength (vorticity) will be 
presented. The processes of vortex formation in fluid dynamics and particularly within the left ventricle during diastole will be explained. Then the effect of left ventricle diseases on vortex formation will be described. As it will be explained, in patients with abnormal heart function, the intra-ventricular flow is disrupted causing the geometry of the vortex to change and can also generate undesirable vortices. The presence of two types of vortical flow structures, a vortex at the sharp edge of the mitral anterior leaflet in healthy subjects and additional vortices at the vicinity of the apex of the left ventricle in patients will be discussed.

Moreover, the current available non-invasive vortex imaging techniques and tools for both clinical and experimental applications will be introduced. Finally, goal and objectives in this research study will be clarified. To that end, Doppler vortography modality for intra-ventricular vortex imaging will be described in chapter 3. Because the Doppler velocities have specific symmetry in the vortex position, in this chapter an index called "blood vortex signature" (BVS) reflecting the intra-ventricular vortices during early filling will be proposed. In addition, a vortex is mainly described by its core vorticity and quantifying the intraventricular vortices could be of clinical interest. Therefore, the core vorticities as a function of Doppler velocities will be accurately estimated. In silico and in vitro experiments will be performed to validate our technique and to analyze the effect of transducer position and kernel window size. Then, the results of the preliminary pilot study on 18 patients including normal subjects and hypertensive patients will also be presented. At the end our results, followed by the technical limitations and future developments of the proposed technique will be discussed.

Due to the novelty of this research study in the field of cardiology, my results were presented at 3 conferences (IEEE Ultrasonics Symposium 2011, Canadian Cardiovascular Congress 2012, and Congrès Étudiants CRCHUM 2011, 2012) in oral and poster presentations. My findings were also accepted for publication in "Ultrasound in Medicine \& Biology" journal which is a high impact factor journal in the domain of echocardiography. Conference proceeding and journal paper are presented in the appendix section for additional information. 


\section{CHAPTER 1 INTRODUCTION}

In this chapter, we give an overview of the heart physiology and briefly discuss on the heart function. Some basic principles of medical ultrasound imaging are also described.

\subsection{Heart and left ventricle}

The heart is one of the most vital organs in the body. It is composed of muscle tissue, the myocardium, which acts to pump the blood carrying oxygen and essential nutrients to the body tissues and similarly rid the body tissues of waste, transporting these materials to their appropriate site for disposal [3, 4]. The circulatory systems functions in a cyclic manner with the heart playing the central role. The cycle starts with returning deoxygenated venous blood from the body which collects in the right atrium via the superior and inferior vena cava [3, 4]. From the right atrium the blood passes through the right atrioventricular valve to the right ventricle. Each of the cardiac valves in blood circulation serves the functional purpose of maintaining unidirectional flow $[3,4]$.

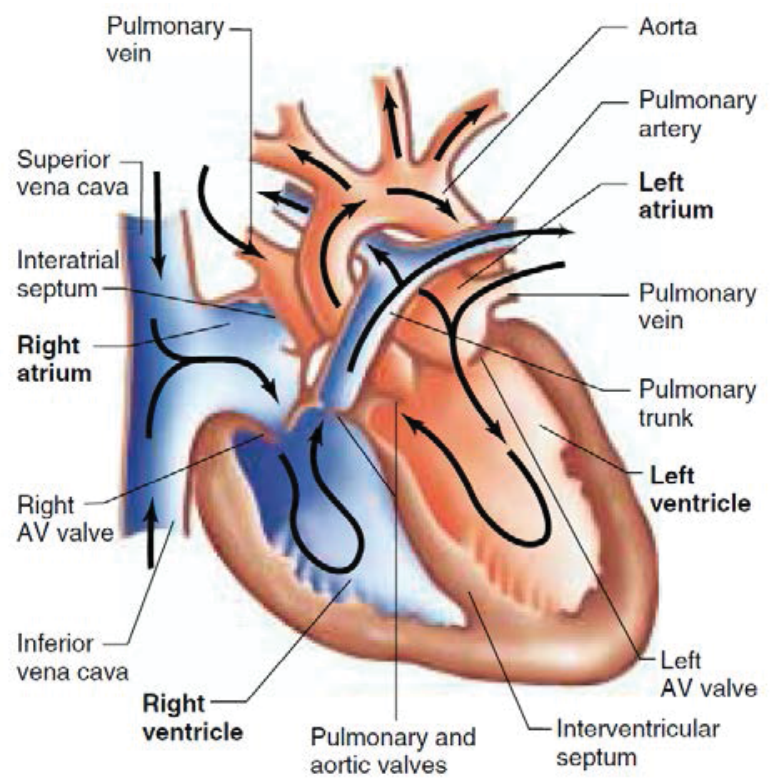

Figure 1-1: Sequence of blood circulation in a normal heart [3]. 
Upon filling of the right ventricle the blood goes to the lungs, via the pulmonary valve, to release carbon dioxide and become oxygenated for recirculation, once again, to the body. The pumping action to the body occurs on the left side of the heart. The left atrium fills with blood, which moves to the left ventricle, via the mitral valve, and is ejected into the aorta via the aortic valve. This sequence is demonstrated by Figure 1-1 [3, 4].

The left ventricle is considered to be a high pressure pump. Because the left atrial receives the blood passed through the pulmonary circulation at a pressure about 10 $\mathrm{mm} \mathrm{Hg}$ and empty itself in to the left ventricle. After left ventricular contraction the blood pressure rises to around 100 to $140 \mathrm{~mm} \mathrm{Hg}$.

The anatomy of the heart has a complex physiology. For proper cardiac function there is an imperative coupling between electrical and mechanical activity that must be maintained.

\subsubsection{Cardiac cycle}

The contraction of the heart is driven by the aforementioned electrical functioning. As both sides of the heart contract in essentially an identical manner and the left ventricle has the majority of the pumping power, it will suffice to discuss the electromechanical coupling with respect to the left ventricle of the heart. The cycle of the heart is divided into two major phases: systole, for which the ventricle contracts and blood is ejected into the aorta, and diastole, for which the ventricle is relaxed and filling.

Starting from the beginning of diastole, the ventricle begins relaxation in preparation for filling (phase 5 in Figure 1-2). The beginning of this relaxation period is called isovolumetric ventricular relaxation (IVR). As we see in Figure $1-2$, the pressure in the ventricle is less than that of the aorta yet greater than that of the atrium, so relaxation is performed with both valves closed [3, 4]. Then the slight increase in size of the ventricle caused by relaxation, while maintaining the same volume, causes the pressure to decrease rapidly. At the point for which the ventricular pressure dips below the pressure in the atrium, the mitral valve opens 
and beings ventricular filling (phases 6 and 7 in Figure 1-2). The pressure in the ventricle will match the pressure in the atrium for the remainder of diastole $[3,4]$. The next marked event occurs due to the depolarization of the atria (phase 1 in Figure 1-2), for which the atrium will contract with a small delay due to the implementation of muscle fibre mechanisms [3, 4]. The contraction of the atria forces the last filling stage of the ventricle at which time the mitral valve is partially closed due to the reversed pressure gradient. At approximately the same moment as atrial contraction begins the ventricle begins to depolarize [3, 4]. This delay as seen in the previous section is attributed to the time it takes for the electrical signal to propagate from the atrioventricular node to the Purkinje fibres allowing time for the atria to contract fully and complete the filling of the ventricle before ventricular contraction starts. As in the case of relaxation, ventricular contraction also begins with an isovolumetric contraction stage (phase 2 in Figure 1-2) whereby the ventricle begins to contract, shown by a steep increase in ventricular pressure in Figure 1-2, until the pressure in the ventricle reaches the pressure of the aorta. At this point the aortic valve will open and full contraction and ejection of the ventricle occur (phases 3 and 4 in Figure 1-2) [3, 4]. 


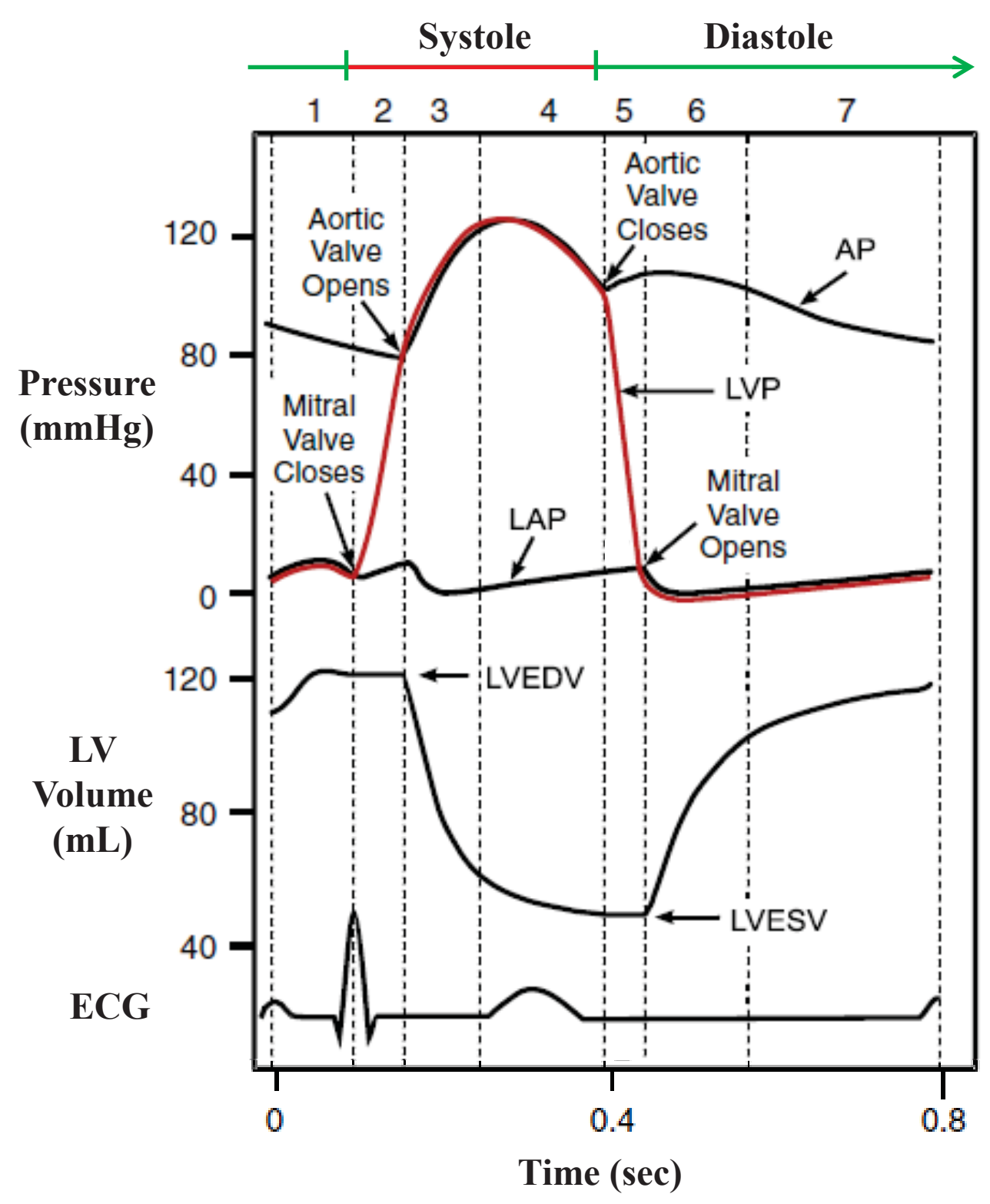

Figure 1-2: Events of the cardiac cycle involving the left heart [5]. Different phases of cardiac cycle are as follows: 1) atrial contraction, 2) isovolumetric contraction, 3) rapid ejection, 4) reduced ejection, 5) isovolumetric relaxation, 6) rapid filling, and 7) reduced filling. LV, left ventricle; ECG, electrocardiogram; AP, aortic pressure; LVP, left ventricle pressure; LAP, left atrial pressure; LVEDV, left ventricle end diastolic volume; LVESV, left ventricle end systolic volume. 


\subsubsection{Mitral valve}

One of the important and complex components of the heart is the mitral valve. The mitral valve is an atrioventricular valve which connects the two chambers of the left heart, the left atrium and the left ventricle. The mitral valve is a thin dual-flap valve with asymmetric leaflets and anterior and posterior cusps. The anterior leaflet is typically larger in area than the posterior leaflet and is located between the mitral orifice and the left ventricle outflow tract. The posterior valve, the smaller cusp, is positioned in the contralateral side. The mitral leaflets are connected to Chordae tendineae which is attached to the papillary muscles at the bottom and sides of the left ventricle [3-5].

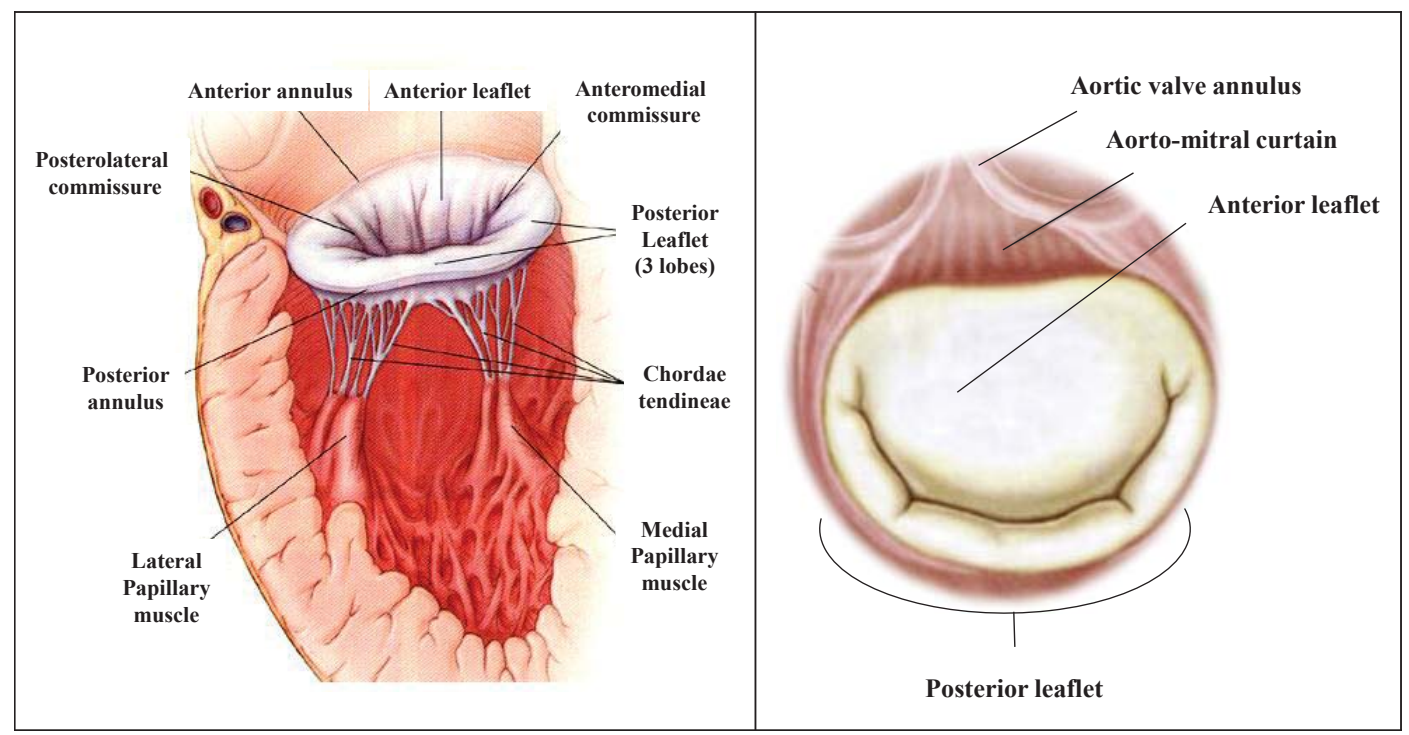

Figure 1-3: Side view (left) and top view (right) of the mitral valve [6].

The mitral valve opens during diastole allowing the blood to enter the left ventricle from the left atrium and closes during systole to prevent reverse flow (leak) from the left ventricle to the left atrium. During systole, while the left ventricle contracts, the annulus dilates and the chordae tendineae hold up and seal the leaflets in case of high pressure and hinder the blood from entering the left atrium. 


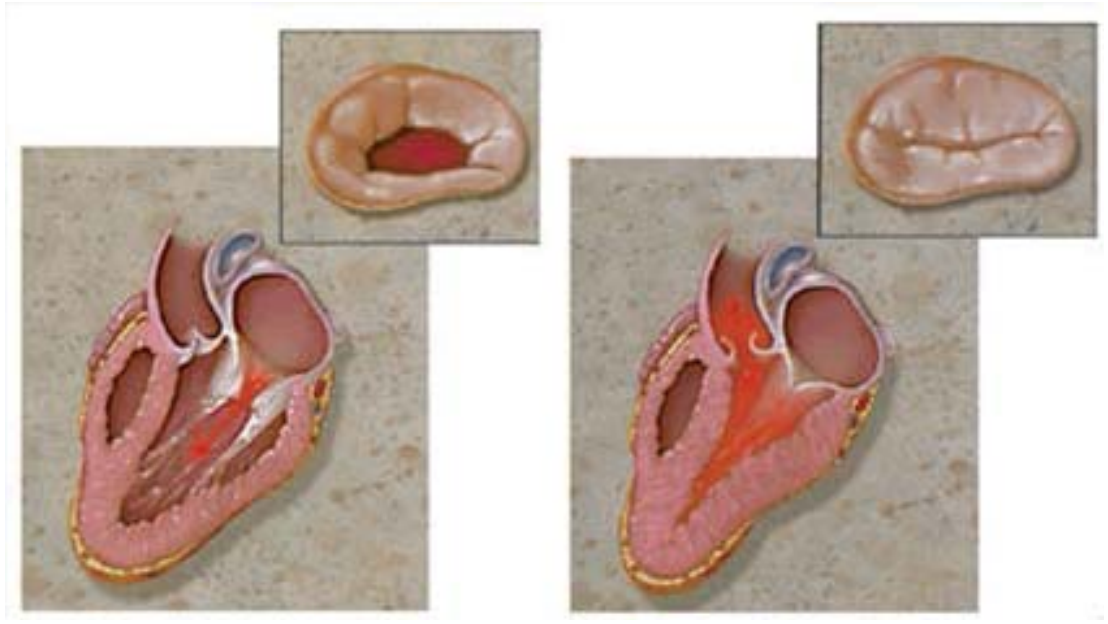

Figure 1-4: The mitral valve function during diastole (left) and systole (right) [7].

\subsection{Heart failure}

Heart disease is one of the three leading causes of death in Canada [8, 9]. Heart failure is the most rapidly growing cardiovascular disease that will impact the lives of Canadians during the next decades [10]. It develops due to functional or structural cardiac disorders that impair the ability of the heart to meet the oxygen demands of tissues. Heart failure affects approximately 400,000 Canadians per year (equating to one heart failure every minute) and the incidence continues to rise due to the aging population and the dramatic prevalence of chronic cardiovascular diseases. The prevalence of heart disease is expected to double by 2025 [11]. Heart failure (HF) significantly contributes to cardiac mortality and morbidity and has a major impact on public health care costs, including physician services, hospital costs, lost wages and decreased productivity [12]. Cardiovascular diseases cost the Canadian economy approximately 20 billion dollars per year [13]. 


\subsubsection{Systolic and diastolic dysfunctions}

Most patients with HF have symptoms related to an impairment of the myocardial function of the left ventricle (LV) [12]. Heart failure can be categorized into two different abnormalities: systolic and diastolic dysfunctions which are respectively due to insufficient contraction and relaxation of the heart [14-16].

One of the good clinical indicators of the ventricular systolic function is the left ventricle ejection fraction (EF). Left ventricle EF measures the percentage of blood pumped out of LV in each heartbeat. It can be calculated by the difference between LV end diastolic volume (EDV) and LV systolic volume at end systole (ESV) divided by EDV. However, about half of patients with diagnosis of heart failure have preserved left ventricular systolic function with a normal or near normal ejection fraction $[15,16]$.

In this research study, only the intra-ventricular flow patterns during diastole are considered. Thus, in this section we mostly focus on diastolic function as well as the different ways for its assessment. The clinical definition for diastolic heart failure constitutes the presence of heart failure symptoms and signs while maintaining a normal ejection fraction (ejection fraction greater than 45\%) and systolic function [17]. In the case of diastolic dysfunction, the relaxation process is abnormally delayed followed by a decrease in LV pressure drop during early diastole. Therefore, the minimum LV diastolic pressure is achievable in a longer time interval than normal conditions which in turn delays the blood flow suction from the left atrium into the LV. Consequently, it increases the time taken to reach an equilibrium pressure between LV and LA. Due to this delayed relaxation, the atrial contraction would be responsible for the considerable portion of the diastolic filling. Under this condition, the left atrium may lose its compliance and as a result the LA pressure increases at the end of diastole. The elevated LA pressure impairs the myocardial relaxation in resting state in severe diastolic dysfunction [18]. When the relaxation process is reduced at rest, the ventricle is also unable to relax fully to fill with blood in an adequate diastolic filling period in case of exertion or stress. The abnormal relaxation at rest is followed by a lack of atrial contraction to 
compensate the remaining ventricular filling process in severe diastolic dysfunction; this results in an elevation in LV diastolic pressure. Therefore, in diastolic dysfunction the heart is able to pump sufficient oxygenated blood to the body, but at a higher filling pressure [18]. Higher LV end-diastolic pressure causes the LA to eject the blood backward into the pulmonary veins. Then excessive accumulation of blood in the pulmonary circulation is expected which leads to right heart failure [18]. Besides, a significant reduction of the cardiac output and stroke volume is observed in severe diastolic dysfunction because the LV becomes so rigid that it disturbs the normal atrial muscle functioning and subsequently the EDV cannot match with the abnormal elevated filling pressure [17, 19].

\subsubsection{Available diastolic parameters}

\subsection{End diastolic pressure volume relationship (EDPVR)}

Pressure-volume (PV) loop is a primary powerful tool to quantify cardiac mechanisms and ventricular function. It provides pressure measurements against volume measurements within LV in a heart cycle. During ventricular filling, the pressure generated at a given volume can be determined by compliance of the LV. Left ventricle compliance can be determined by the ratio of a change in volume divided by a change in pressure. In Figure 1-5, left ventricle filling (a), isovolumetric contraction (b), LV ejection (c), and isovolumetric relaxation (d) corresponds to a single cardiac cycle are represented. Information regarding EDV, ESV, and stroke volume can be easily obtained by PV loops [5]. 


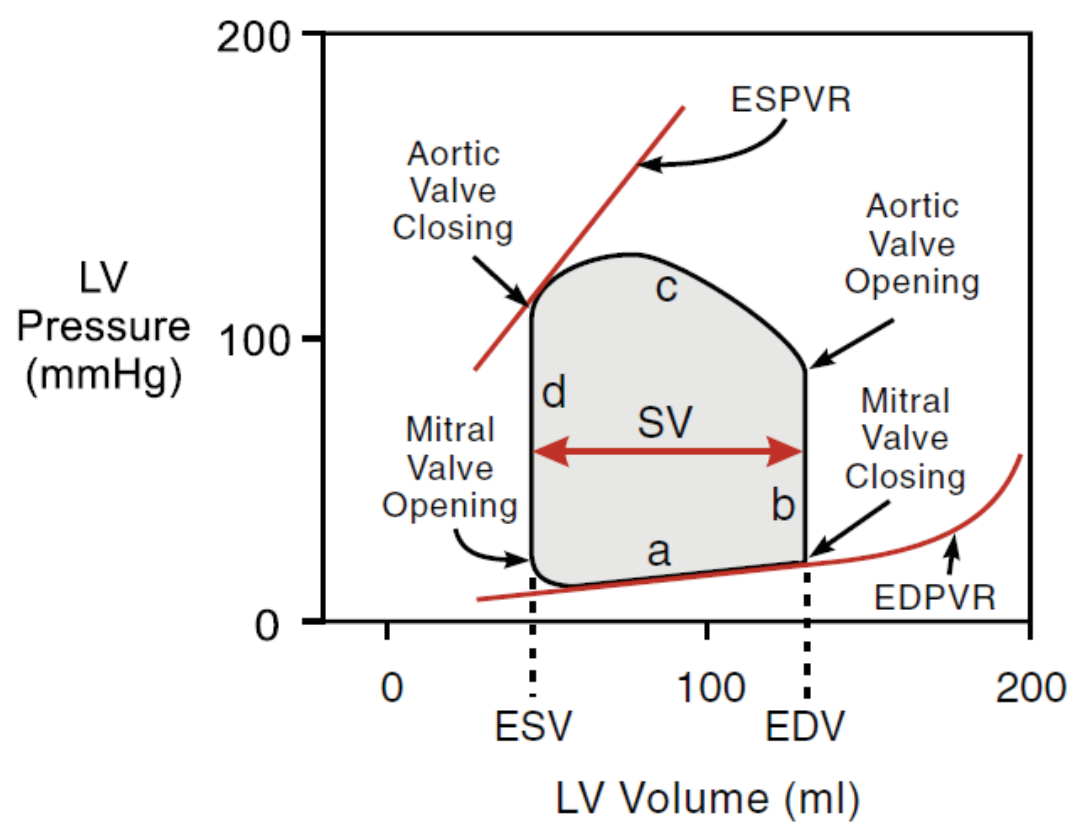

Figure 1-5 : LV pressure volume loop which represents ventricular pressure against ventricular volume at any given point in a single cardiac cycle. Different period of a heart cycle is represented by letters. a, ventricular filling; b, isovolumetric contraction; $c$, ventricular ejection; $d$, isovolumetric relaxation. ESV, end systolic volume; EDV, end diastolic volume; SV, stroke volume; EDPVR, end diastolic pressure volume relationship; ESPVR, end systolic pressure volume relationship [5].

As it can be seen, the filling phase can be described by end diastolic pressure volume relationship (EDPVR). The slope of the EDPVR at any point along the curve is reciprocal of LV compliance. Pressure and volume follow a nonlinear relationship within the LV. Hence, the slope of the filling curve increases (LV compliance decreases) with increasing pressure and volume. For instance, in hypertrophic patients the LV compliance is decreased which results in higher LV end diastolic pressure at any given EDV. Hence, EDPVR slope will increase in a way that the filling curve shifts upwards and to the left (refer to Figure 1-6). In dilated heart, larger EDV without causing a great elevation in end diastolic pressure is expected and the filling curve will shift downwards and to the right (reduction in EDPVR slope as shown in Figure 1-6). However, providing pressurevolume loops require invasive pressure-volume catheter-based measurements [5]. 


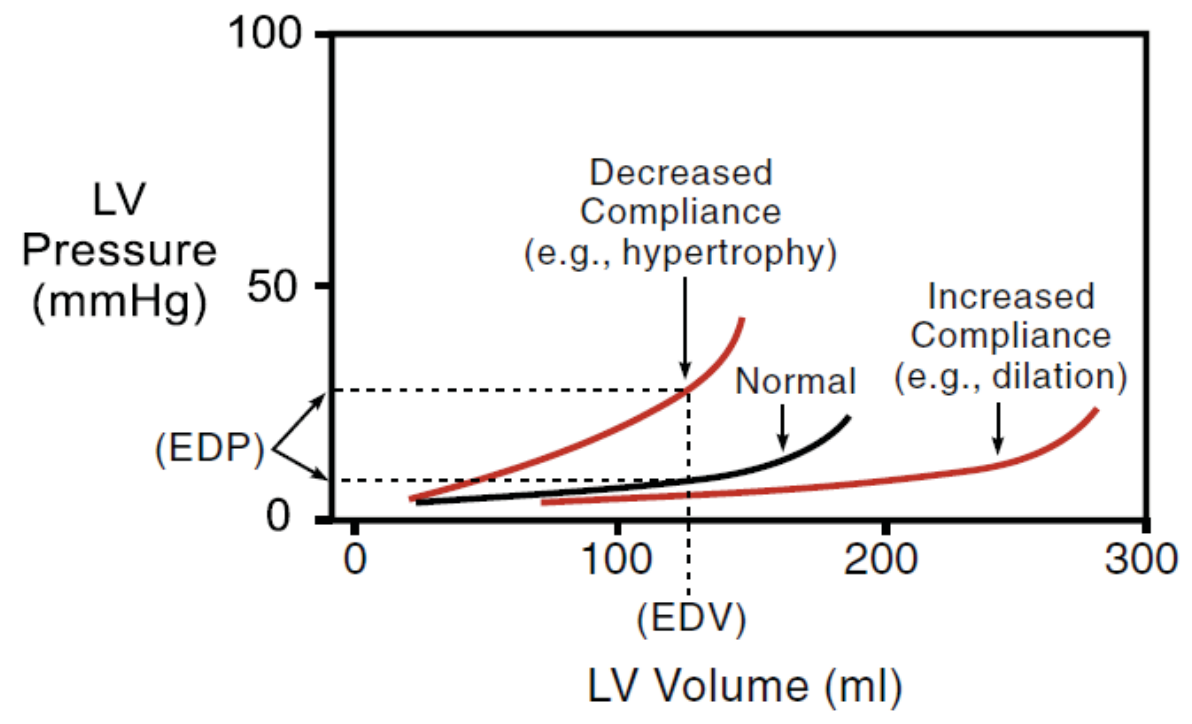

Figure 1-6: Ventricular passive filling curve (EDPVR) which provides an insight into the ventricular diastolic function. The EDPVR for normal, hypertrophic, and dilated left ventricles are represented. The slope of the EDPVR increases in hypertrophic patients, however it decreases in the presence of ventricular dilation. LV, left ventricle; EDV, end diastolic volume; EDP, end diastolic pressure [5].

There also exist some echocardiographic (basic principle of echocardiography will be discussed later in this chapter) measurements for the evaluation of left ventricular diastolic function. Most commonly used echocardiographic parameters are as follows.

\subsection{Mitral annular movement $\left(e^{\prime}\right)$}

A reliable parameter to evaluate the LV myocardial relaxation is the velocity of mitral annular movement ( $\mathrm{e}^{\prime}$ ) during early diastole which can be measured by tissue Doppler echocardiography $[18,20]$. In normal young subjects in resting state, septal and lateral $\mathrm{e}^{\prime}$ are greater than 10 and $15 \mathrm{~cm} / \mathrm{s}$ respectively. During physical activities or in case of stress, these relaxation parameters increase showing that the LV is able to achieve a lower minimum LV pressure to increase early diastolic filling. However, $\mathrm{e}^{\prime}$ will be decreased in patients with diastolic dysfunction. Therefore, mitral annular movement $\left(\mathrm{e}^{\prime}\right)$ is the primary parameter in 
the assessment of the LV diastolic function [18]. However, it has been shown that mitral annular movement parameter is preload dependent [15].

\subsection{Early wave velocity and late velocity ratio (E/A)}

Another essential parameter for the evaluation of the LV diastolic function is primary measurements of the mitral inflow velocities recorded by Doppler echocardiography. Normal mitral inflow consists of rapid filling wave at the onset of diastole phase after mitral valve opening (E-wave) and late diastolic filling wave after atrial contraction (A-wave) [18]. In healthy subjects the early diastolic mitral inflow velocity (E-wave) is higher than the late velocity (A-wave) after atrial contraction; the E/A ratio is greater than 1 . With advancing age, the diastolic dysfunction can be defined by lower $\mathrm{E}$ velocity than the $\mathrm{A}$ velocity $(\mathrm{E} / \mathrm{A}<1)$ followed by a longer deceleration time as well as a reduction in $\mathrm{e}^{\prime}$. In advanced stages of diastolic dysfunction, the early diastolic mitral velocity (E) increases with a short deceleration time and the $A$ velocity decreases $(E / A \geq 2)$ followed by a reduced $\mathrm{e}^{\prime}$. In intermediate stages of diastolic dysfunction, $\mathrm{e}^{\prime}$ is reduced but with a normal mitral inflow velocity parameters (E/A > 1) [18]. However, mitral inflow velocities are load dependent and also affected by other factors. Therefore, some patients with severe diastolic abnormality may show a pseudonormal filling pattern [21].

\subsection{E/e' ratio}

Of note, correcting E velocity with the effect of relaxation parameter $\mathrm{e}^{\prime}$ can reveal its relation with LA and LV filling pressures [18]. The E/e' ratio is correlated well with the mean pulmonary capillary wedge pressure (PCWP) obtained by simultaneous pulmonary artery catheter measurements [18, 20]. The PCWP can provide an indirect measure of the LA filling pressure. In case of diastolic dysfunction with elevated filling pressure the E velocity increases and relaxation $\mathrm{e}^{\prime}$ decreases; thus the $\mathrm{E} / \mathrm{e}^{\prime}$ ratio increases [18]. According to the literature, patients with E/e' ratio lower than 8 are considered as individuals with normal LV filling pressure and the ones with E/e' ratio higher than 15 are considered as patients with 
elevated LV filling pressure [18]. However, there are some clinical settings in which E/e' ratio should not be used to evaluate diastolic function. For instance, in healthy subjects E/e' ratio may not give a reliable estimate of the ventricular filling pressure [15].

\subsection{Mitral flow propagation velocity $\left(V_{p}\right)$}

The mitral flow propagation velocity $\left(\mathrm{V}_{\mathrm{P}}\right)$ measured by color $\mathrm{M}$-mode Doppler is also able to estimate LA filling pressure and reflect the myocardial relaxation. It behaves as a non-invasive index for the prediction of LV filling pressure and the assessment of LV diastolic function. Combining the $\mathrm{E}$ velocity with $\mathrm{V}_{\mathrm{P}}$ provides a better estimation of PCWP and more importantly the diastolic function. In vivo studies in patients with diastolic dysfunction show that $\mathrm{E} / \mathrm{V}_{\mathrm{P}}$ and $\mathrm{LA}$ pressure are directly proportional to each other so this ratio is able to obtain estimation of LV filling pressures $[15,18,20,22]$. However, patients with abnormal filling pressures but normal LV volume and EFs can have deceptively normal $\mathrm{V}_{\mathrm{p}}$. Moreover, it has been previously shown that $\mathrm{Vp}$ is preload dependent in patients with both normal and depressed EFs [15].

\subsection{Current diagnostic tools for left ventricle dysfunction}

\subsubsection{The electrocardiogram (ECG) tracing}

Electrocardiogram tracing is the primary clinical tool to diagnose rhythm disturbances of the heart as well as changes in the electrical conductivity of the myocardium. In cases of myocardial ischemia and infarction, ECG is a key tool to diagnose the disease. ECG is able to identify the extent, location and also the progression of damages in the ischemic or infarcted areas. In Figure 1-7 the component of the ECG trace can be observed. The P-wave, QRS complex and Twave represent the atrial depolarization, ventricular depolarization and ventricular repolarization respectively. 


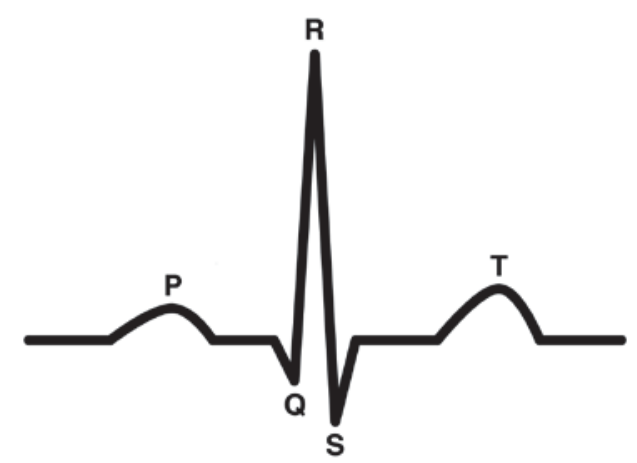

Figure 1-7: The components of a normal electrocardiogram (ECG). P: atrial depolarization; QRS: ventricular depolarization; T: ventricular repolarization.

The continuous repetition of waves in the ECG trace represents the continuity of the depolarization and repolarization of the atria and ventricles [23]. Abnormal Qwave, T-wave, or ST-segment and also any changes in the shape of the QRScomplex reveal myocardial infarction or ischemia. However, it is possible to obtain a normal ECG in cases of ischemia, infarction or cardiac arrest. In such cases, the electrical activity of the heart (ECG tracing) seems normal, but the heart does not contract efficiently or for any reason, the cardiac output is inadequate to transport blood to all organs of the body [23].

\subsubsection{Magnetic resonance imaging}

Magnetic resonance imaging (MRI) is regarded as gold standard in non-invasive imaging and evaluation of cardiac and vascular function. Magnetic resonance imaging provides an accurate assessment of the anatomic characteristics of the heart (size of the chambers, thickness of the walls, valve function, degree and extent of the cardiac disorder) and the peripheral blood vessels [24]. Magnetic resonance imaging can also be used for a thorough quantitative cardiac flow and evaluation of valvular function. High quality images can be acquired by means of $3 \mathrm{D}$ cine phase contrast magnetic resonance imaging which synchronizes the imaging with respiratory and cardiac movements [24, 25]. 3D cine phase contrast velocity data acquisitions can provide useful information on the time-resolved characterization of blood flow in cardiac chambers. Furthermore, 3D time- 
resolved MRI has the potential to provide arbitrary cut-planes which are not accessible by echocardiographic imaging. However, MRI cannot be part of routine clinical set-up. The measurement time to acquire images by MRI technique is highly dependent on the size and accuracy of the measured data, heart rate and also the efficiency of respiration control for each patient [24]. For instance, the time required for acquiring a complete heart velocity data set with a spatial resolution of $3 \times 3 \times 3 \mathrm{~mm}^{3}$ and temporal resolution of $46 \mathrm{~ms}$ by $3 \mathrm{D}$ time-resolved MRI is $20 \mathrm{~min}$ [24]. Although, it is possible to speed up the acquisition time by decreasing the number of samples, this may generate artefacts which complicate data processing.

\subsubsection{Doppler echocardiography}

Echocardiographic imaging is the primary routine clinical diagnostic tool in cardiology. Echocardiographic imaging can provide plenty of invaluable information about anatomic distances, volume measurements, motion study, cardiac pumping capability, and also the severity of tissue damages. In addition, it can be used to estimate the heart function such as evaluating the cardiac output and diastolic function. The wide accessibility of cardiac ultrasound and its ability to derive real time non-invasive information makes echocardiography the prerequisite technique for the evaluation of cardiac diseases such as hypertrophied and dilated cardiomyopathy.

Color Doppler imaging is an accurate echocardiographic technique for visualizing the blood flow through the heart and the cardiovascular system. It uses standard echographic methods to provide an overview of flow patterns. It combines anatomical information obtained by pulse-echo ultrasound with velocity information derived using Doppler ultrasound techniques to generate a colorcoded map of blood velocity superimposed on a gray scale image of tissue anatomy. During color flow acquisitions, a transducer is passed over the skin, above the target area (heart chambers or some parts of the cardiovascular system). The probe emits multiple small pulses of ultrasound beams which reach the 
sampling area and return to the probe. The received signal gives information on the blood flow in the area of interest. The flow velocity can be estimated from the phase shift between the echoes at a fixed depth during two or more subsequent pulses (refer to section 1.7) [26-28]. Given its wide availability, non-invasive, radiation-free and low-cost nature compared to other imaging methods such as CT and MRI, Doppler echography is currently the method of choice to evaluate cardiac diseases. Ultrasound imaging is a highly valuable technique in this context as it can achieve the high frame rates necessary to assess the cardiac function and blood flow visualization.

While evaluating the cardiac function, it is essential to work with a data set which provides the true velocity information of the myocardium movements and blood flow in the heart chambers. However, using ultrasound in this context has a number of weaknesses. Its main limitation is that only one dimensional blood flow measurements (only velocity components along the transducer scanline towards or away from it) are obtained in clinical practice, while ideally it must include information regarding both the magnitude and the direction of three dimensional velocity vectors. Moreover, the blood flow assessment may be hindered due to the presence of strong surrounding tissue signal. In cardiac application the clutter from the heart walls and surrounding tissue is much higher than echoes from the blood particles; it precludes the accurate velocity data. The presence of ultrasound beam side lobes and reverberations also reduces the image spatial resolution and contrast of the images. So in order to provide an accurate diagnosis of the heart function, special attention should be made to prevent the clutter effect during ultrasound acquisition throughout the cardiac cycle [26, 27]. 


\subsection{Basic physics of ultrasound}

\subsubsection{Propagation of ultrasound waves}

The sound we normally hear is from 15 to 20,000 cycles per second. Ultrasonic waves are sound waves that have higher frequencies than the audible frequency range which causes mechanical disturbances and vibrations in materials. Medical ultrasound uses typically a 100 times higher frequencies than the limit of human hearing which is 2 to $20 \mathrm{MHz}$. When a force is applied normal to the medium surface, a longitudinal pressure wave is generated in which the movement of the particles in the medium is parallel to the wave propagation direction. In the presence of longitudinal pressure waves, compression and rarefaction zones (Figure 1-8) can develop in the medium. The compression and rarefaction of sound waves can be generated in liquids as well as solid mediums with the speed of propagation dependent on the medium properties. In solids, the sound can also propagate as shear waves or transversal waves in which the particles oscillate at a direction perpendicular to the direction of propagation. Clinical ultrasound imaging most widely uses longitudinal wave propagation because shear waves are rapidly attenuated in tissue $[29,30]$.

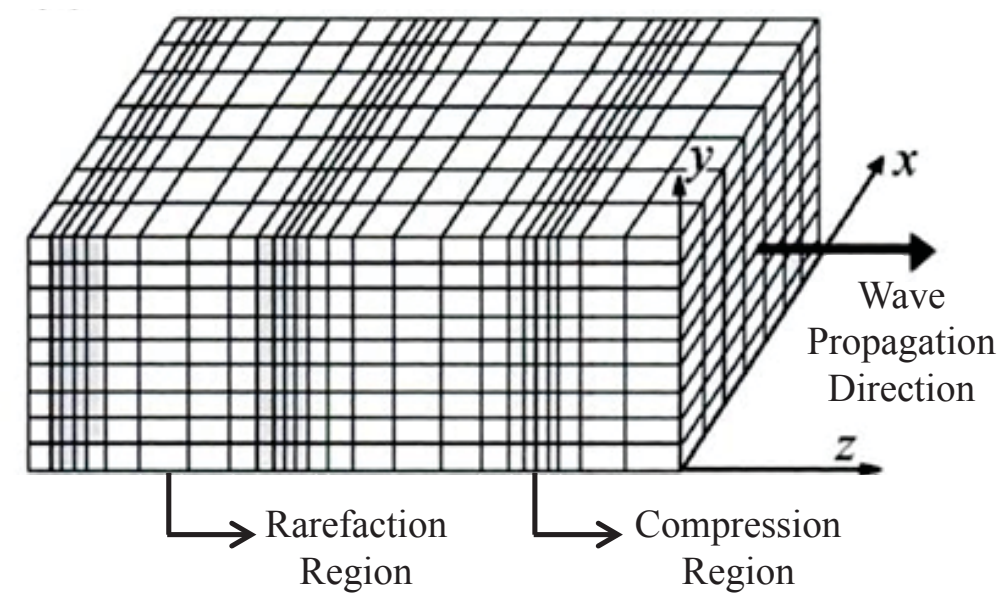

Figure 1-8: Propagation of longitudinal waves, consider the rarefaction and compression zones [31]. 
The speed of longitudinal ultrasound waves is determined by the ratio of the bulk modulus $(\mathrm{K})$ and the density $(\rho)$ of the medium. The bulk modulus is the substance's resistance to uniform compression and is inversely proportional to the compressibility $[29,31]$.

$c=\sqrt{\frac{K}{\rho}}$

This equation shows that compressibility and speed of sound are inversely proportional. For instance, the sound in air travels at a greater speed than in skeletal bones. The speed of sound in most tissues in the human body is considered to be $1540 \mathrm{~m} / \mathrm{s}$ [30].

\subsubsection{Scattering}

When the ultrasound energy propagates in a medium, various interactions of ultrasound waves with matters including reflection, refraction, scattering, and absorption are expected due to the changes in impedance of the medium [26, 27, $29,32]$.

When the incident beam is perpendicular to the tissue boundary, a portion of the beam, after interaction with the boundary, returns to the transducer as an echo. The remaining portion of the beam continues transmitting through the medium in its initial direction. This phenomenon in which the dimension of the smooth boundary is larger than the incident wave length is called specular reflection. The larger the difference in impedance, the greater the reflection will be. Bones and vessel walls are the most regular structures that generate specular reflections. These types of reflections are not desirable in cardiovascular imaging and will be compensated by clutter filtering $[29,32]$. 


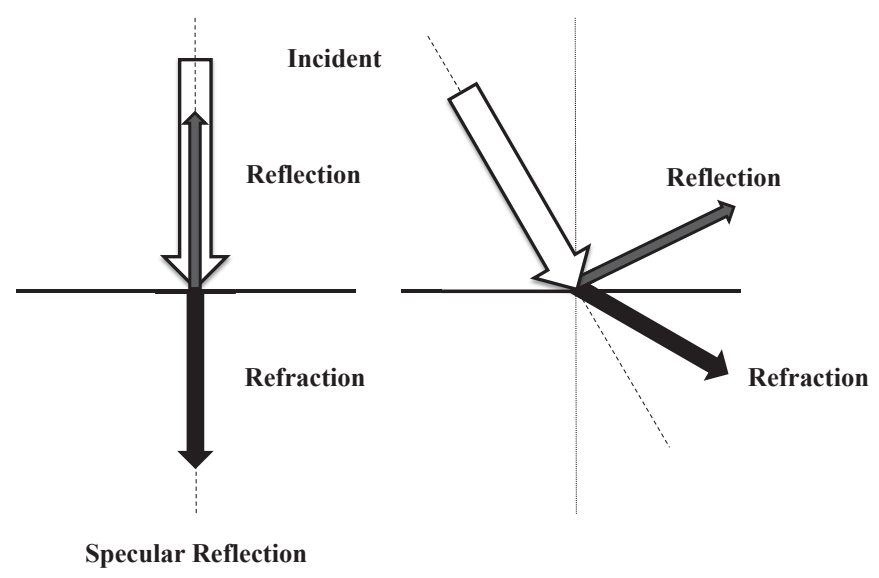

Figure 1-9: Reflection and refraction of ultrasound occurs at the tissue boundaries. With perpendicular incident to a boundary, a portion of the beam is transmitted and a portion of it is reflected back to the probe $[29,32]$.

Refraction occurs in cases of non-perpendicular incidence beams where the direction of the transmitted sound wave on a tissue interface changes. In this case the frequency does not change, but the speed of sound does [29, 32].

Scattering is reflection or refraction that causes some of the echoes to diffuse in multiple directions due to contact with small particles (at about the size of the wavelength or smaller) or rough surfaces in the medium. The part of the scattering beam that returns back to the source (the backscattered waves) increases the characteristic texture and brightness changes on the acquired image and is considered to be the main contributor to medical ultrasound imaging [29, 32, 33]. In a medium with the inhomogeneity smaller than the ultrasound wavelength, diffusive scattering occurs. Scattering from blood is an example of diffusive scattering. The liquid component of the blood, plasma, consists of red blood cells, white blood cells and platelets. Red blood cells account for about 45 percent of the blood volume in a healthy adult. White blood cells and platelets represent about $1 \%$ and $0.2 \%$ of the blood volume respectively. Considering that red blood cells are the most abundant in the blood, the effect of backscattering from white blood cells and platelets is negligible. The shape of red blood cells is a biconcave disk with flattened center and an approximate diameter of $7 \mu \mathrm{m}$ and thickness of $2 \mu \mathrm{m}$. 
The frequencies normally used for ultrasound imaging in clinical practice are between 2 to $15 \mathrm{MHz}$ [30]. The wavelengths corresponding to these frequencies range from $0.77-0.1 \mathrm{~mm}$ (the higher the frequency the shorter the wavelength $\lambda=$ $\mathrm{c} / \mathrm{f}$ ) and are much larger than the diameter of red blood cells [30]. This results in diffusive scattering.

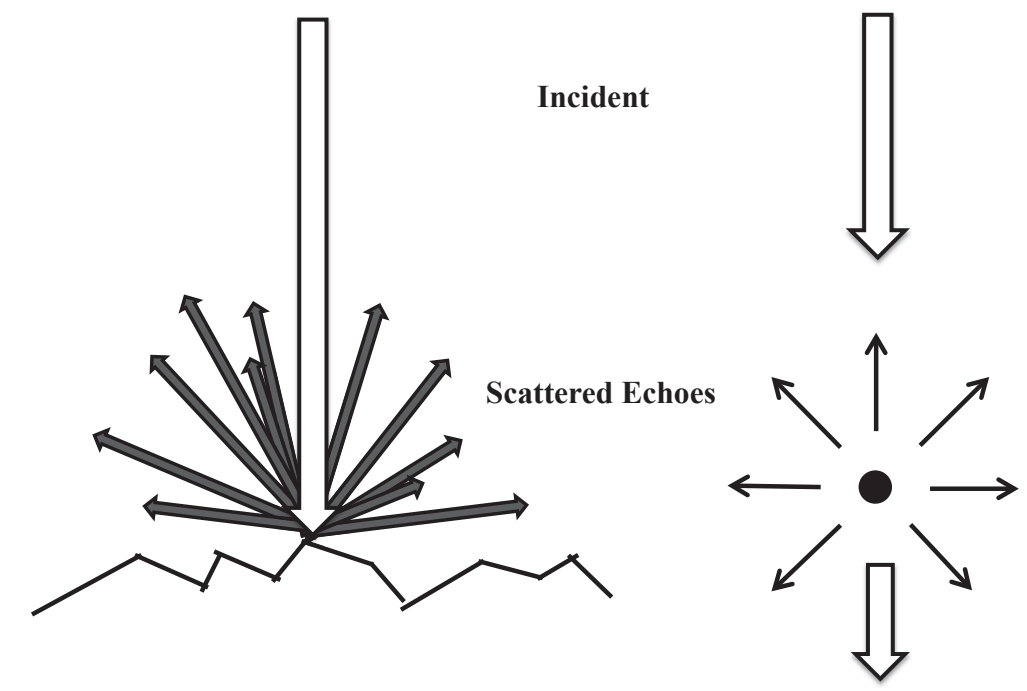

Figure 1-10: Illustration of the scattering of the ultrasound wave in contact with a small particle or rough surface $[29,32]$.

High frequency probes produce shorter wavelengths which result in strong specular reflections from red blood cells. It is known that higher portions of ultrasound waves turn straight back to the probe by means of specular reflection. The greater return of the ultrasound waves causes an overestimation of Doppler velocities (hyperechoic images) which is adjusted to the greatest extent by ultrasound attenuation [32].

Absorption occurs when the ultrasound energy is transformed into heat energy. In the presence of absorption, the sound energy is lost as it travels through tissue and cannot be recovered. Absorption in biological tissues appears due to the medium viscosity and particle relaxation time. Higher viscosity results in higher ultrasound absorption in the medium. Relaxation time is the time required for the particles to return back to their initial place after being forced to a new position due to the 
transmission of ultrasound waves. The stress exposed to the particle by the wave is relaxed by thermal relaxation or changing the structural states [29, 32, 33].

\subsubsection{Attenuation}

In practice, there is a loss of acoustic energy as waves propagate further into the tissue. This is known as ultrasound attenuation and is mainly caused by absorption, scattering, non-linear propagation, beam divergence, reflection and refraction. [29] The attenuation coefficient which is the relative loss of beam intensity (absorbed or scattered) per centimetre of travel varies for different tissue characteristics. The ultrasound wave is highly attenuated and scattered in fat tissue, but the scattering decreases in watery medium. The attenuation coefficient in blood is approximately $0.18 \mathrm{~dB} /(\mathrm{MHz} \mathrm{cm})$ which is low in comparison with tissues [29].

In biological tissues ultrasound attenuation is approximately proportional to frequency. Hence, the high frequency components of the ultrasound wave will be more attenuated (amplitude reduction) than the low frequency components. The central frequency of the backscattered ultrasound wave is decreased in comparison to the central frequency of the incident transmitted wave. Consequently, an underestimation in the Doppler velocities is expected. Furthermore, the maximal penetration depth of the ultrasound signals is of high importance when selecting an ultrasound probe and considering its central frequency [29, 32]. To evaluate structures such as the carotid artery, a probe with $7-12 \mathrm{MHz}$ central frequency is required, because it is superficial and located under the muscle layer near the skin surface of the body. Deeper components, such as the heart can be examined by $2-5$ $\mathrm{MHz}$ frequency probes [29, 32]. 


\subsection{Basic imaging principles}

\subsubsection{Pulse-echo imaging}

Conventional ultrasound imaging uses the pulse echo-imaging principle. During pulse echo acquisition, the transducer excites a pulse ultrasound pressure field and emits pulses through the medium. The pulse waves reflect or scatter when any changes in the acoustic impedance of the medium occur. The backscattered signals moving to the probe are RF (Radio Frequency) signals [28]. These frequency bands can be processed to acquire images and characterize the backscattered signals. The time delay between the transmitted and the received RF signal is related to the depth (d) of the reflector based on the following equation:

$d=\frac{c t}{2}$

Note that $\mathrm{c}$ is the speed of the ultrasound wave, and the constant 2 refers to the round-trip travel of the pulse from and to the transducer. Since the speed of sound in tissue is high, approximately $1540 \mathrm{~m} / \mathrm{s}$, real time ultrasound image acquisition is possible.

\subsubsection{Transducer}

Transducers are devices which excite and receive the pressure waves to and from the medium. The ultrasound probes are made of piezo-electric materials which are able to convert electrical energy to acoustic energy. The piezo-electric material generates an electrical signal when exposed to mechanical stress (vibration), and on the other hand, vibrates when the applied electrical field is changed. The transducer uses these two properties for the reception and transmission of ultrasound pressure signals for image acquisition [29, 32].

An ultrasound transducer consists of many individual rectangular piezoelectric elements arranged in linear or curvilinear arrays (Figure 1-11). 

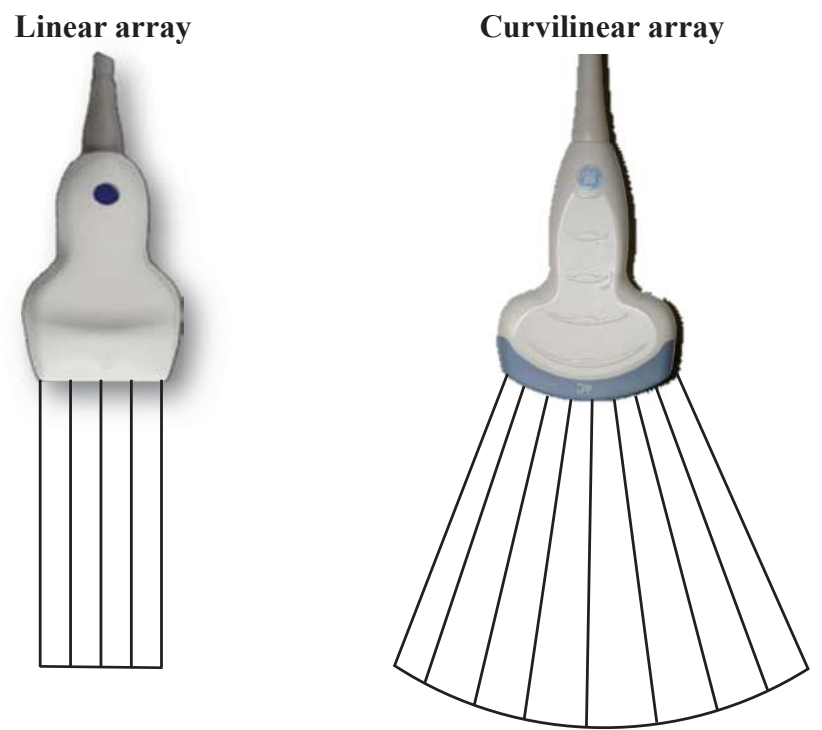

Figure 1-11: Linear and curvilinear ultrasound transducers [29].

There is a small gap between the elements of the transducer known as Kerf. The space between elements is filled with acoustic isolating materials to avoid crosstalking. The distance between the centres of the transducer elements is called the pitch. Each ultrasound transducer has 64 to 512 rectangular elements with a width less than a half of the wavelength and a length in the millimetre range depending on the transducer type. Transducers can have up to 512 elements with widths of 75-120 millimetres. The beam produced by such a narrow element will diverge rapidly after the wave travels only a few millimetres. The smaller the transducer face, the more divergent the beam will be. This leads to poor lateral resolution (section 1.8.1) and low sensitivity due to beam divergence and small element size $[29,34]$. The solution to overcome this problem is to fire 8 to 16 adjacent elements simultaneously [29]. To form an ultrasound beam, a number of transducer elements must fire in such a way that the inner element pulses are delayed with respect to each other. These electronic delays assist to line up the transmitted pressure signals from all parts of the aperture to reach the field point concurrently; applying correct delays is called beam focusing. The depth of focus for the transmitted beams is determined by the pulsing time delays and can be changed during ultrasound acquisition. To obtain a dynamic beam focusing, the same delay 
factor can be applied for the returning pulses from the area of interest. The same pattern is repeated for the next set of elements with one element shifted from the previously used elements [29,34].

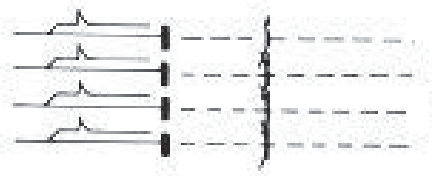

Synchronous

beam

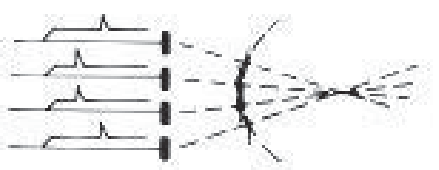

focused

beam

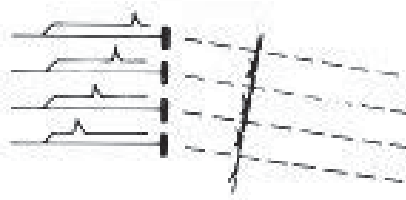

beam

steering

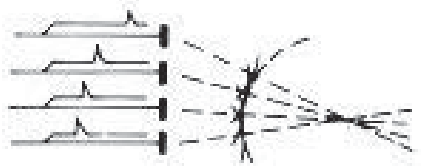

steering and focusing

Figure 1-12: Implementation of delay to control beam focusing and steering [34].

Figure 1-12 explains the process of beam steering and focusing in an ultrasound image acquisition. As it can be seen, for emitting a synchronous beam (figure top), all transducer elements fire simultaneously, without time delay. By using time delays in the electrical activation of adjacent elements, the ultrasound beam can be steered and focused without moving the transducer. In the focused beam (figure second from top) the peripheral beams have further distances to travel to the desired position and thus are fired earlier to maintain the same distance in that time period. Hence, for the focused beam the delay curve has a parabolic shape. Similarly, for beam steering (third from top) the first emission occurs for the element that is furthest to the transducer face. Therefore, the delay curve has a linear progression. Finally, the fourth image (figure bottom) defines the 
combination of steering and focusing beam, using the same delay principles [29, 34].

There are different types of multi-element transducer arrays, including linear array and phased array transducers (Figure 1-13). Linear array transducers consist of 256 to 512 elements. In this type of transducer, a subset of the total number of elements (e.g. 20 adjacent elements) fire simultaneously to produce the ultrasound beam. To acquire rectangular ultrasound images a continuous emission of the ultrasound pulses from other groups of excited transducer elements displaced by one or two elements is required [29, 30].

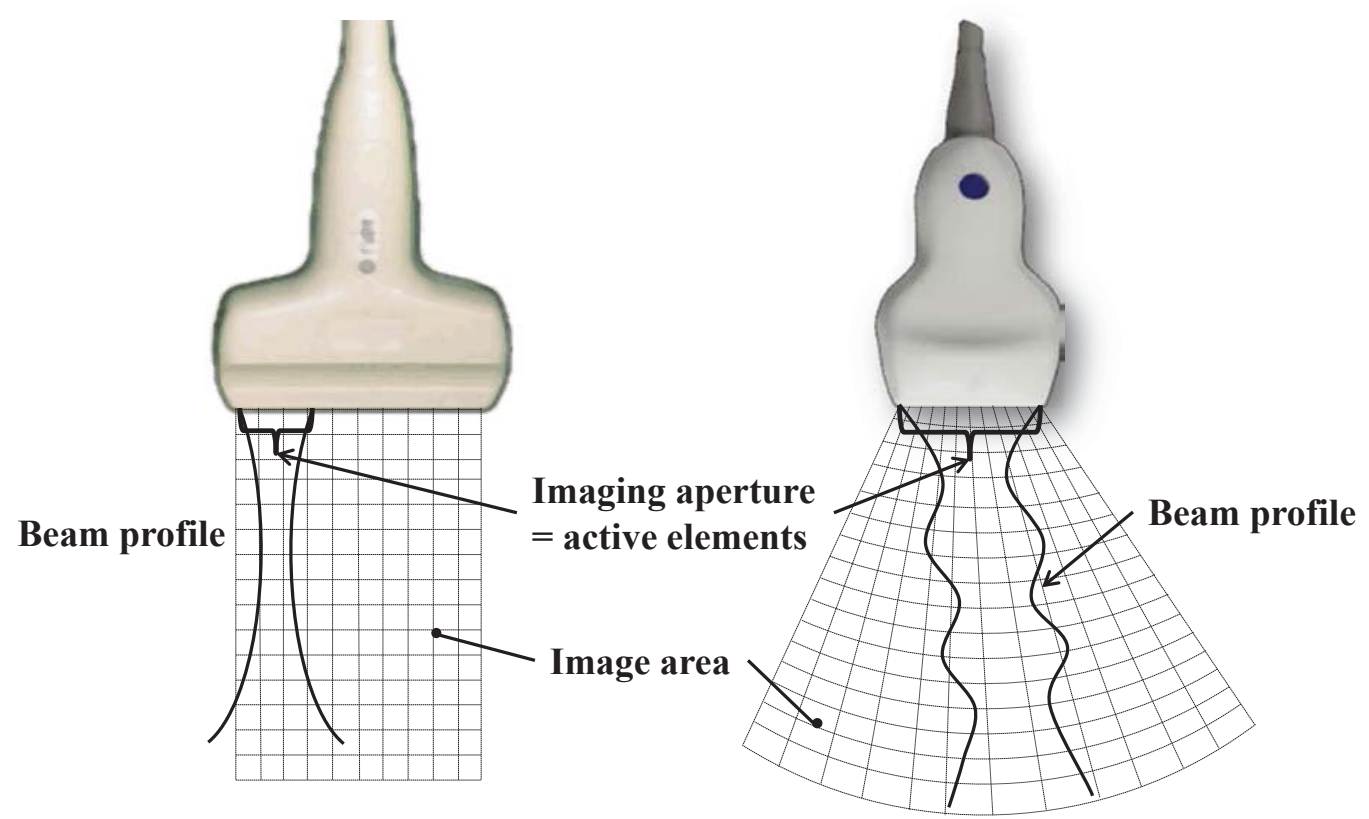

Figure 1-13: Illustration of a linear array and phased array transducer [30].

In the case of imaging the heart, linear arrays are too large to be positioned between ribs. Phased array probes with smaller array and larger field of view are suitable for cardiological investigations through the ribs. A phased-array transducer usually consists of 64 elements in which all elements are activated simultaneously to produce a single cone-shaped image [29, 30]. 


\subsection{Echo display modes}

Various methods are used to display the information obtained from ultrasound acquisitions. The most common imaging modes are M-mode, B-mode and color Doppler mode [29].

M-mode is an ultrasound imaging technique that displays echoes from a moving structure such as the myocardium and heart valve leaflets. In this technique, the anatomy only along a single scan line versus time is acquired from a stationary probe and is displayed in a curve with scatter depth on the vertical axis and time on the horizontal axis. [29]

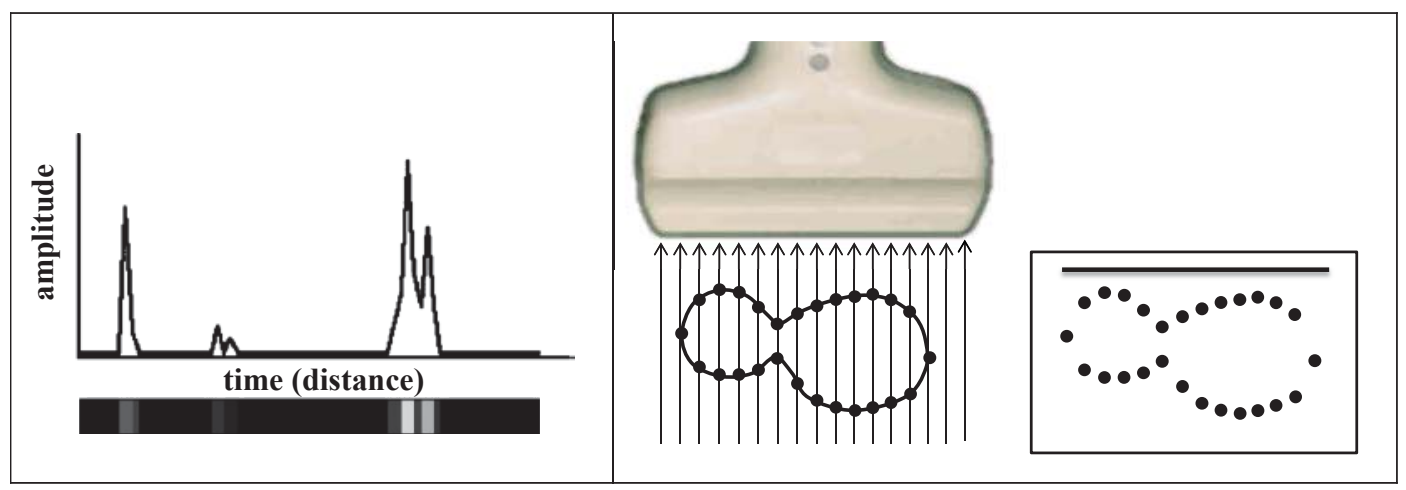

Figure 1-14: B-mode scanning principle based on the displaying the echoes as bright dots in a time-base line [35].

B-mode (grayscale imaging) is the most frequently used ultrasound diagnostic tool for visualization and quantification of anatomical structures. B-mode imaging is a 2D ultrasound imaging system composed of brightness modulated dots in which the amplitude of the reflected sounds are represented by the brightness of the dots (refer to Figure 1-14 and Figure 1-15). The position of dots in the time baseline is related to the time required for the echoes to reach the transducer's surface $[29,30$, 35]. 


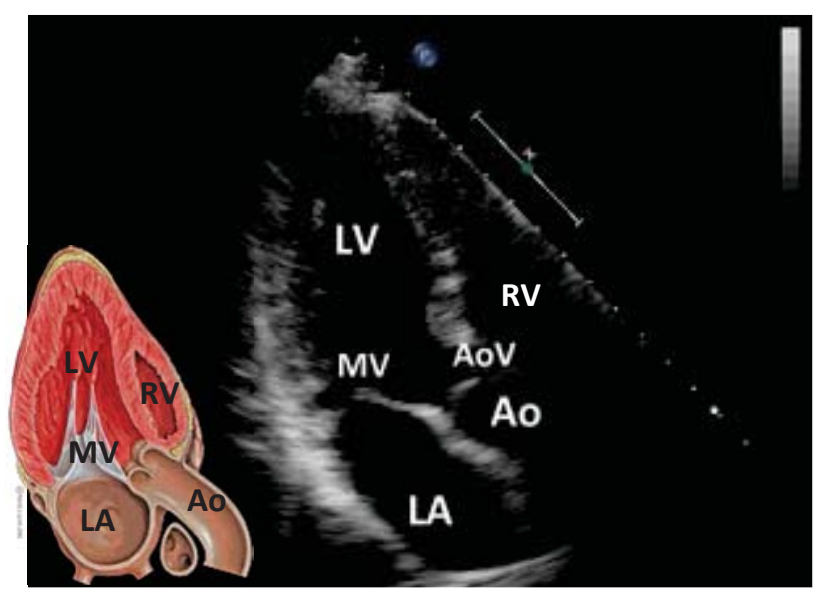

Figure 1-15: An example of a B-mode scanning of a normal heart in an apical 3-chamber view [36]. LV, left ventricle; RV, right ventricle; LA, left atrium; MV, mitral valve; AO, aorta; AOV, aortic valve.

Color flow imaging uses the standard ultrasound methods to produce a colorencoded map of Doppler shifts representing the velocity and direction of the blood flow. The map is superimposed onto a real-time B-mode gray-scale of soft tissues (the myocardium or the blood vessels) (refer to Figure 1-16).

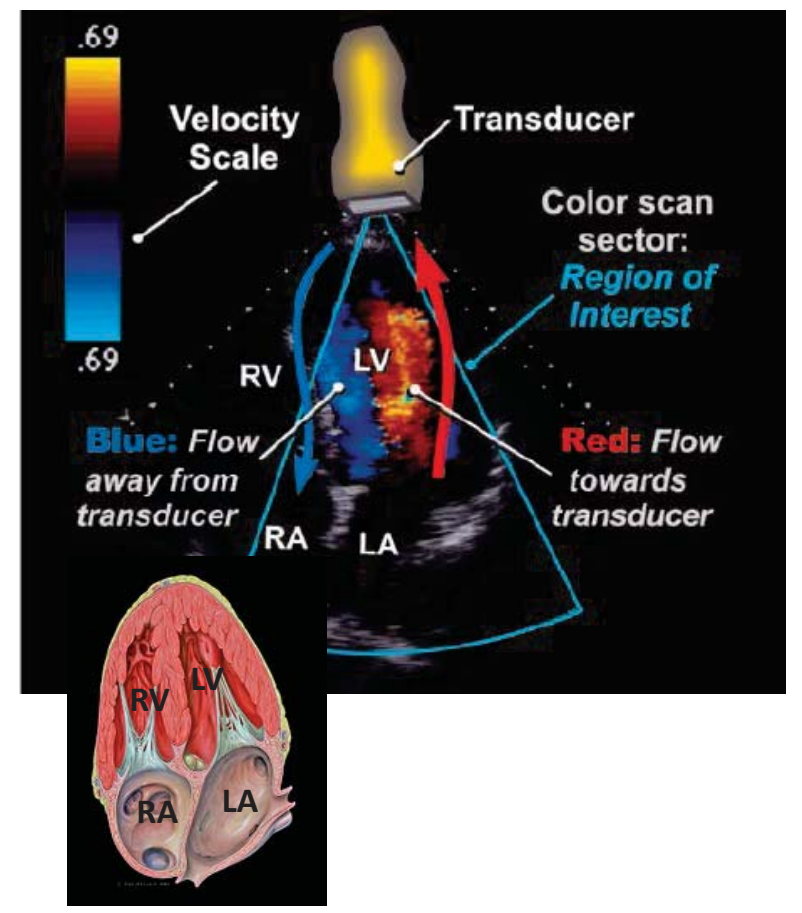

Figure 1-16: Color flow Doppler imaging in an apical 4-chamber view of a normal heart during diastole [37]. LV, left ventricle; RV, right ventricle; LA, left atrium; RA, right atrium. 


\subsection{Diagnostic ultrasound imaging}

The different steps for diagnostic imaging in a conventional ultrasound machine are represented in Figure 1-17. In this section we briefly discuss the algorithms, processing requirements, and challenges of the common diagnostic ultrasound modes, such as B-mode and color flow imaging.

The ultrasound probe emits various short ultrasound pulses with 4 to 8 cycles by converting the electrical signals with 2 to $10 \mathrm{MHZ}$ using a piezoelectric transducer [30]. The transducer fires ultrasound pulses with the pulse repetition frequency (PRF), varying from 0.5 to $20 \mathrm{KHZ}$, based on the required time to reach the maximum depth of interest [28]. As it is mentioned before, the acoustic pulses travel through the tissue, a portion is scattered back to the transducer when any changes in acoustic impedance (such as tissue boundaries) are met. The received pulses which give information on amplitude, frequency and phase content of the backscattered signals are converted into RF electrical signals [28, 30].

As the acoustic waves are attenuated while passing through the medium, the transducer first amplifies the received signals by time gain compensation (TGC) (Figure 1-17a). Time gain compensation is an automated amplification applied as a function of time to correct the effect of acoustic attenuation by the tissue medium. The TGC is proportional to the depth travelled by the beam into the tissue $[28,29$, 38]. Once the radio frequency signal is amplified by the TGC, it passes through an analog to digital converter (A/D) (Figure 1-17b) [28]. The A/D is an electronic device whose function is to sample the continuous signal at a high rate (typically between 20 to $40 \mathrm{MHz}$ ) in order to represent the signal in digitalized discrete time units. The sampling frequency, $\mathrm{f}_{\mathrm{s}}$, represents the rate at which the digital samples are taken from the continuous analog signal. Then the demodulator (Figure 1-17c) multiplies the sampled digital radio frequency data by the cosine and sine function to remove the carrier frequency and recover the received signal. In-phase quadrature demodulation is an electronic process which after low pass filtering results in complex samples of the signal, referred to as IQ-signal, represented by $\mathrm{I}(\mathrm{t})+\mathrm{iQ}(\mathrm{t})$, where $\mathrm{I}$ is the in-phase portion of the signal and $\mathrm{Q}$ is the quadrature 
component of the signal after demodulation [39]. There are two different steps to follow for blood flow estimation: wall filtering and velocity estimation [28, 30].

In blood flow applications, Doppler data is composed of two major signals: high frequency (around $15 \mathrm{KHz}$ ) low amplitude signals from blood motion and low frequency (less than $1 \mathrm{KHz}$ ) high amplitude signals from the motion of the tissue and blood vessel walls [40]. The higher the amplitude of the signal, the heavier the influence on velocity estimation [41]. The signal from wall motion is much stronger than the signal returned from the blood, which is undesirable for color Doppler imaging. In order to acquire accurate velocity estimations of blood flow, this unwanted clutter signal is removed using a high pass filter or clutter filter (Figure 1-17d) [28].

Color flow velocity estimation can be performed by phase-shift technique (Figure $1-17$ e) which is one of the most common velocity estimation methods in conventional Doppler systems. In this technique the in-phase quadrature echo data is converted to phased shift from signals at a fixed depth by means of the autocorrelation given by the following equation $[28,30,38]$,

$\Delta \varphi=\arctan \left(\frac{\sum_{i=1}^{N_{c}-1} Q_{i}(t) I_{i+1}(t)-I_{i}(t) Q_{i+1}(t)}{\sum_{i=1}^{N_{c}-1} I_{i}(t) I_{i+1}(t)-Q_{i}(t) Q_{i+1}(t)}\right)$

where, $N_{C}$ is the total number of sequential pulses for a particular range of pulses, $\mathrm{i}$ and $i+1$ represent a particular scan-line and the subsequent one at the same position. This calculation is repeated for all sampled points taken in the axial and lateral directions to obtain a 2D image of phase shift for each frame. The velocity of a sample volume, such as blood, with respect to the beam direction is calculated by the following equation $[28,30]$ :

$V=\frac{-c * P R F}{4 \pi \cdot f_{s}} \cdot \Delta \varphi$ 


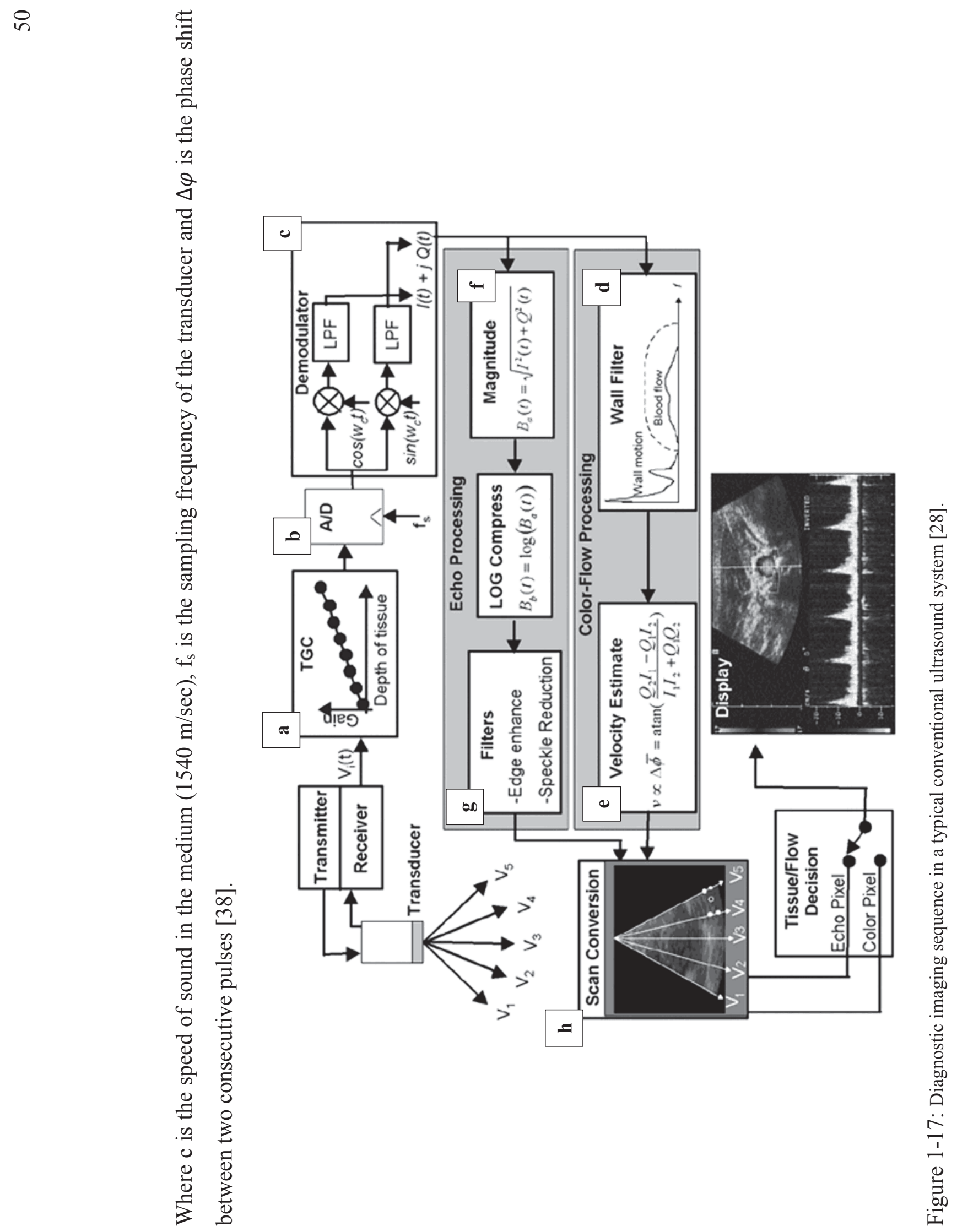


The information given in the complex IQ-signal can also be used for echo processing to provide real time imaging of the various tissue structures in the body and acquire anatomical B-mode images (Figure 1-17f). The brightness or intensity pixel data in B-mode gray scale images can be obtained by constructing the envelope of the RF signal using the complex IQ-signals. The following equation [28] shows the magnitude of the in-phase and quadrature data, the signal envelope, for gray scale representation,

$B(t)=\sqrt{I(t)^{2}+Q(t)^{2}}$

The data is then compressed logarithmically to convert the signal, $\mathrm{B}(\mathrm{t})$, from the sampling range to a small dynamic range proportional to the logarithm of the original input signal [28].

There are different techniques to improve image quality. Edge-enhancement (Figure $1-17 \mathrm{~g}$ ) is used to enhance the different tissue boundaries although it increases noise in the image. Various methods of noise reduction exist which vary from system to system [28]. In cardiac application, the next step after obtaining and processing color Doppler or B-mode images, is to transform and superimpose the polar coordinate data onto a sector scan in a 2D Cartesian image by means of a process called scan conversion (Figure 1-17h). Scan conversion is essential in order to display images in a format compatible with video display monitors.

\subsection{Image quality}

The main sources of artifact and distortion in ultrasound image acquisitions include equipment variables selected by the operator such as transducer frequency, PRF, ultrasound intensity and TGC [33]. Image quality can be measured by calculating the spatial resolution, contrast resolution and image noise. These image artifacts act to degrade the quality of the ultrasound image [29]. 


\subsubsection{Spatial resolution}

Spatial resolution is composed of three different components: axial, lateral and elevational resolution. The axial resolution also known as longitudinal resolution is the minimum separation distance required between two scatters in the direction of the beam to produce separate echoes in an image. The axial resolution is dependent on the pulse length; the shorter the pulse length, the better the axial resolution. For instance, if the distance between the two scatters is bigger than one half of the pulse length, the received echoes from the medium will be clear and without overlap [29, 32]. The resolution in the lateral and elevational direction is dependent on the beam width in and out of the imaging plane, reflector depth and beam focusing $[29,32]$. The overall image resolution can be specified by the point spread function (PSF), which is the image response of a small infinite point reflector. In real systems, the image of the point scatter is blurred and the degree of blurring is a measure of the quality of the imaging system [33].

\subsubsection{Contrast resolution and signal-to-noise ratio}

Noise is generally generated by the transducer, the electronic amplifier and the random nature of the background medium. As previously discussed, the TGC is an essential process that compensates for the depth attenuation in Doppler ultrasound imaging. On the other hand, applying TGC as an amplification process, results in an increase in image noise. Although some image processing solutions may increase the contrast resolution of ultrasound images, they also decrease the frame rate and the spatial resolution. The image contrast reflects the specific differences in echo amplitudes. The level of echo amplification is inversely proportional to the transmit gain; the lower the operation power (the transmit gain), the higher the required signal amplification. Hence, a weak signal amplitude from the medium results in a reduction in the image contrast resolution [29].

As previously discussed, the received signal is depth dependent due to the attenuation. The signal-to-noise (SNR) ratio which characterizes the quality of the 
signal's strength relative to the background noise is also depth dependent. SNR can be calculated by the following formula,

$S N R=\frac{\text { Received signal power }}{\text { Noise power }}$

High quality ultrasound images require a high SNR; the higher the SNR is, the better, as less background noise is received.

\subsubsection{Aliasing}

Aliasing appears when the sampling rate is inadequate to provide clear detection of phase changes without ambiguity. The criterion for the maximum velocity limit is related to the Nyquist theorem, stating that the sampling frequency should be at least two times the highest frequency given in the signal [38]. The maximum detectable velocity is given by [28],

$V_{\max }=\frac{P R F \cdot c}{4 f_{\max }}$

where PRF is the pulse repetition frequency, $\mathrm{c}$ is the speed of sound and $f_{\text {max }}$ is the maximum Doppler shift.

The time interval between two consecutive pulses must be sufficient for the first pulse to reach the reflector and return back to the transducer earlier than the second pulse is transmitted [29]. If the second pulse is emitted prior to arrival of the first one, the ultrasound machine cannot distinguish between the reflected echoes from both transmitted pulses and causes aliasing. Hence, aliasing occurs when a low pulse repetition frequency is set for low velocity examination such as in the case of venous flow. Also, low velocity components may not be detectable by selecting a high PRF in high velocity investigation [27, 29]. 


\section{CHAPTER 2 BACKGROUND AND OBJECTIVES}

\subsection{Literature review}

\subsubsection{Vortex and vorticity definition}

Vortex is the swirling motion of flow around its centre. In this section the origin of the vortex and its formation process in terms of vortex dynamics will be discussed. Then formation of vortices at the edge of the left heart valve leaflet will be briefly described. At the end, the vorticity definition will be explained. In order to identify the origin of vortices, obtaining some knowledge on boundary layer theory in fluid dynamics is required. It is known that in the presence of viscosity, flow close to the boundary has zero relative velocity. The region where flow velocity rapidly increases from zero, at the solid wall, to a value comparable to the outer flow velocity, is called the boundary layer. The vortex formation starts with the boundary layer separation phase. Boundary layer separation from the wall occurs due to the local deceleration of the flow or in other terms due to the presence of adverse pressure gradient in the direction of the flow. The flow deceleration or adverse pressure gradient is most often caused by a change in the geometry. A sharp edge which can be found at the tip of a cardiac valve is an extreme case of geometric change. When flow decelerates at a specific location e.g. a sharp edge, the boundary layer decelerates as well and increases its thickness at the same location (Figure 2-1a). This local thick boundary layer is finally lifted and transported downstream by the outer flow (see arrows in Figure 2-1b). Then, a secondary boundary layer with the opposite rotating direction starts to grow away from the solid wall and cuts the original boundary layer with its backward motion (Figure 2-1c). Thus, the separated clockwise vortex detaches the boundary layer and enters the external flow as an independent vortex [42]. Boundary layers can also be explained by the shear layer also called vortex layer. The basic concept of any shear layer is the velocity difference between its two sides. So, boundary layer 
is a vortex layer adjacent to the wall that is generated due to the velocity difference between the outer flow and the fluid attached to the wall. The part of the shear layer away from the wall detaches and moves with a speed higher than the layers closest to the wall. Then the separated shear layer rolls up and forms an isolated vortex behind the sharp edge [42]. For instance, on the trailing edge of heart valves, where the deceleration of flow is local, the boundary layer separation localizes as well and the incidental vortex develops at the sharp edge and leaves the anatomical structure tangentially [42-44]. In other words, the aforementioned boundary layer is a vortex layer close to the wall that is generated because of the velocity difference between the outer flow and the near-wall flow. This vortex layer is also known as shear layer [42]. During the LV diastole, the shear layer between the high-speed mitral jet and the surrounding still fluid rolls up into a spiral shape and forms an isolated intra-ventricular vortex [45].

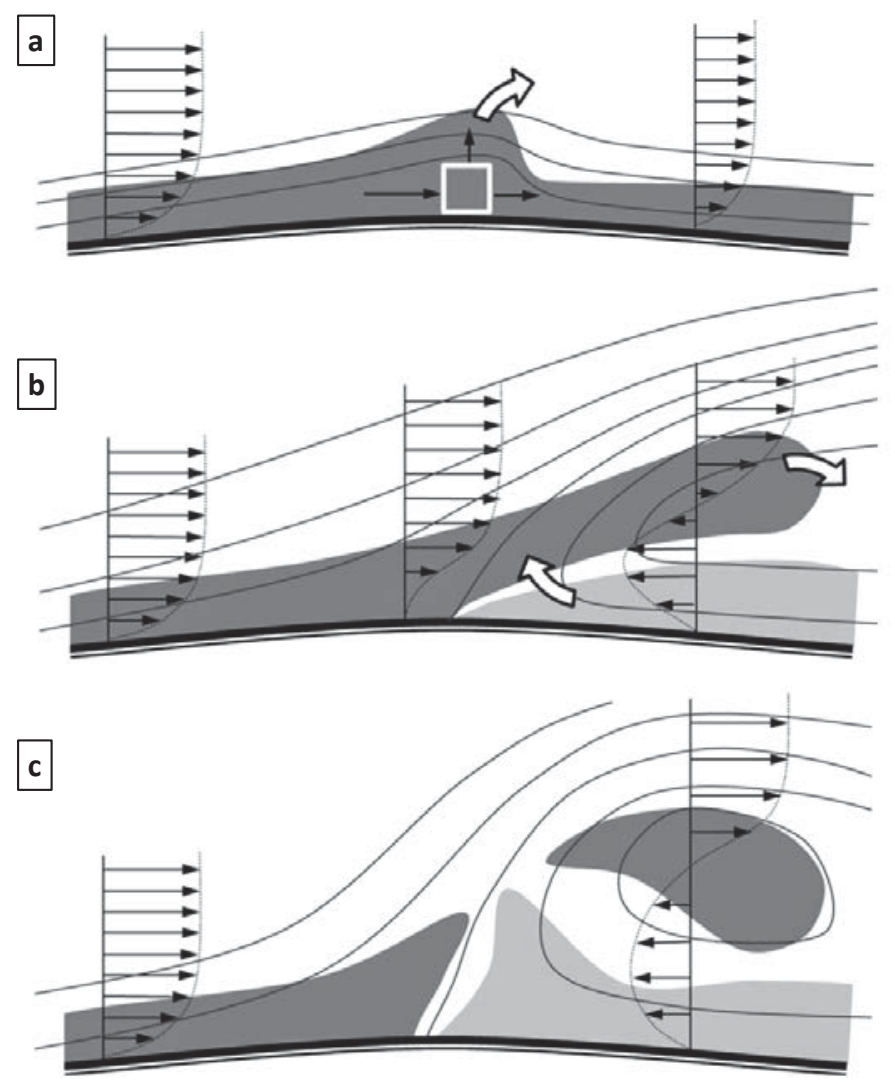

Figure 2-1: The boundary layer separation leading to the vortex formation. Dark and light colors represent the clockwise and counter-clockwise vorticities [42]. 
The tendency of the fluid's particles to spin can be described by a fundamental quantity called vorticity. The vorticity represents the intensity of the vortex and the local rotation rate of the fluid's elements. The vorticity, $\omega(t, x)$, is a vector field defined as the curl of the velocity field $u(t, x)$.

$\omega(t, x)=\nabla \times u$

Vorticity (the curl of the velocity field) is normally measured by its circulation, indicated by $\Gamma$. The circulation of the vortex is defined as the line integral of the fluid velocity within a closed area surrounding a vortex. It is also tantamount to the sum of all vorticities within the circulatory motion area. The vortex with a small circulatory motion area and/or a high circulation has a higher vorticity.

\subsubsection{Vortex formation during diastolic filling}

At the oncoming of diastolic filling, the major flow pattern is the mitral inflow starting jet surrounded by two counter rotating spinning areas (Figure 2-2). The mitral filling jet ejected from the left atrium to the left ventricle feed a large diastolic vortex close to the anterior mitral valve leaflet and a small one near the posterior leaflet. Figure 2-2 illustrates the existence of these vortices around the mitral jet flow during early and late diastole. In a normal left ventricle, the viscous friction between blood flow and the left ventricle wall interferes with vortex formation and changes in the symmetrical shape of the vortices $[1,46]$. During mid-diastole, the asymmetric vortex at the distal end of the anterior mitral valve leaflet grows in size while still being attached to the transmitral flow. During late diastole, this large vortical structure detaches from the transmitral jet and swirls in the direction of natural flow $[47,48]$. 
Early diastole

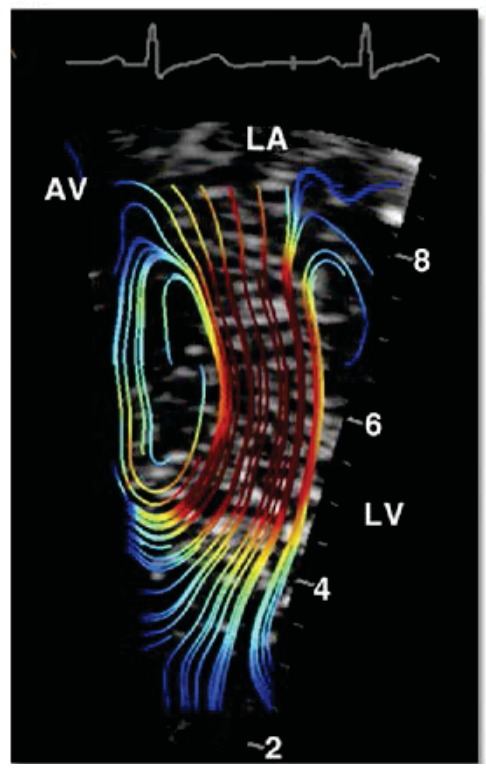

Late diastole

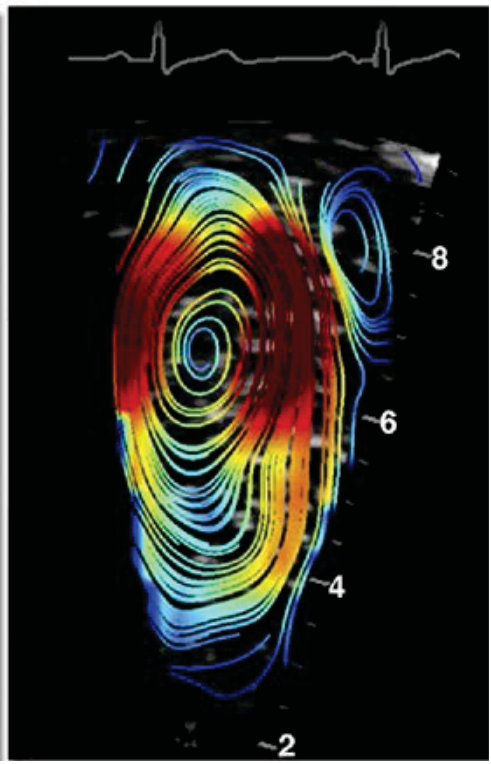

Figure 2-2: Blood flow directions, streamlines, and circulating regions (vortices) in a healthy left ventricle during early (left) and late (right) diastole acquired by Echo-PIV are shown. The kinetic energy of the streamlines is represented by colors. The large anterior and smaller posterior mitral vortices are present on sides of the mitral inflow jet during diastole [49].

Figure 2-3 shows a blood velocity map of a normal left ventricle during both diastole and systole overlaid on the corresponding anatomical B-mode image [47]. The fluid dynamics of the left ventricle filling reveals that the inflow from the mitral valve to the left ventricle attains a maximum velocity of around $1 \mathrm{~m} / \mathrm{s}$ after a short travelling distance of a few centimeters. The blood that entered must turn around following the sinuous curvature of the healthy heart and exit the left ventricle through the aortic valve in a fraction of a second while keeping its initial velocity. As a consequence, a swirling pattern (transmitral vortex) during the diastole phase of the cardiac cycle within the left ventricle is required to redirect blood flow towards the aorta [50]. As it can be seen in Figure 2-3, the mitral swirling pattern could still survive during the early stages of ventricular systole and redirects blood towards the aortic valve while it perishes shortly after systole at the outset of the isovolumic relaxation phase. In this figure the vectors show the 
magnitude and the direction of blood flow velocity. The velocity range is indicated by the colour bar at the right.

Early diastole

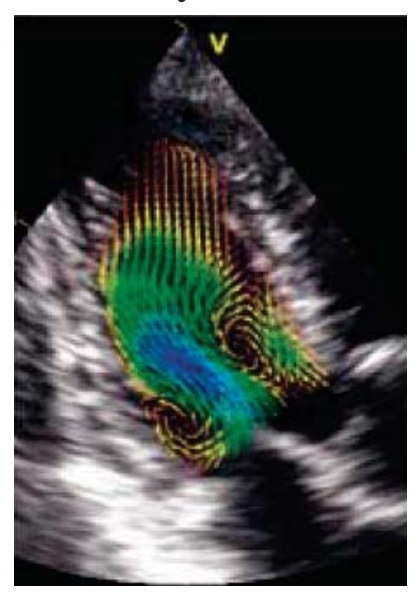

Late diastole

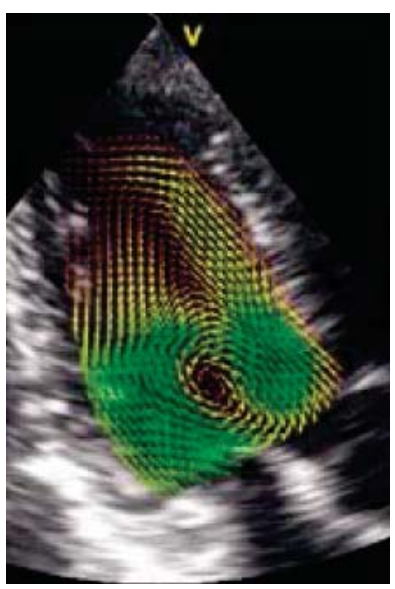

Systole

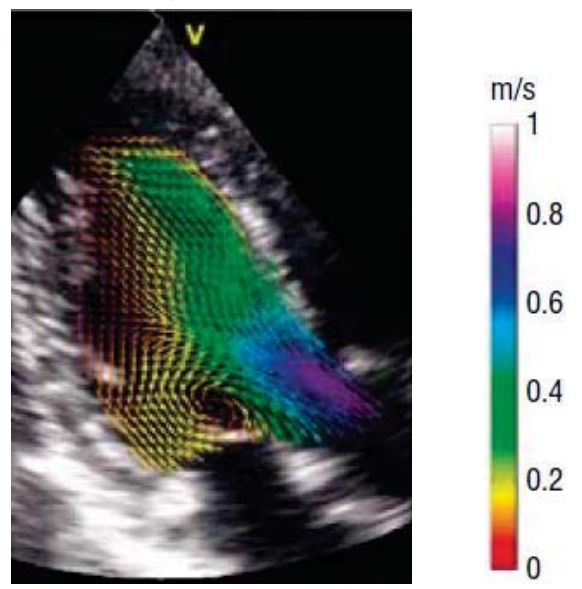

Figure 2-3: The blood velocity mapping of a normal LV during diastole and systole overlaid on the corresponding anatomical B-mode image [47].

In the normal heart, a large part of the left ventricular blood volume is actually involved in vortex formation. The transmitral vortex acts as a source of mechanical energy and ensures the flow transition to ejection which makes systole tightly coupled with diastole $[16,51]$. Recent numerical in vitro and in vivo observations suggest that this diastolic swirling pattern minimizes fluid energy dissipation and optimizes the myocardial pumping efficiency of the left ventricle $[1,2]$. The presence of the mitral vortex allows rinsing of the endocardial surface during systole and avoids blood stagnation and thrombus formation within the left ventricle [52]. In addition, the mitral flow during diastole generates an apical diastolic suction force which maximizes the amount of blood entering the left ventricle and increases the recoiling force at the mitral annulus[48]. Moreover, the anatomical properties of the left atrium and the asymmetric nature of the mitral valve leaflets contributes to the asymmetry of the flow pattern and consequently to the fluid energy dissipation [50]. The mitral orifice eccentricity is an effective parameter in blood flow asymmetric circulation in the LV and also in the ventricular pumping efficiency [2]. The transmitral vortex formation in a normal 
heart keeps the mitral valve leaflets closer to the LV posterior wall and helps blood flow automatic ejection during the systolic phase. For instance, if the mitral orifice is more centrally oriented, energy dissipation will increase because blood flow will not be favourable for ejection from the LV inflow to the outflow at the end of the diastole phase [42]. This may result in a significant increase in cardiac work and oxygen demand.

\subsubsection{Effect of left ventricle diseases on vortex formation}

Vortices that form during diastole have specific time varying positions, geometries, and swirling strengths, known as vorticities [47]. The presence of any specific changes in LV shape or contractile capacity, called ventricular remodeling, results in an abnormal intra-ventricular blood flow pattern [47, 53-59] that is less efficient in blood transportation through the heart cavities $[60,61]$ and may accordingly lead to the progression of heart failure [48]. In addition, the correct formation of the cardiac vortex is highly dependent on the balance between the endocardium wall motion and the blood flow. Any alterations in this natural arrangement may disturb the flow and affect the geometry of the diastolic vortices [62].

For instance, dilated cardiomyopathy (DCM) is a cardiac muscle disorder in which the left ventricular muscles start to stretch and become thinner following by an enlarged and rounded LV chamber. In higher levels of the disease, other heart chambers, the right ventricle and the atria, are also affected. This enlargement prevents the heart from contracting normally and pumping blood efficiently to the body tissues and organs. In patients with dilated cardiomyopathy, due to ventricular enlargement, the flow entering the LV during diastole is directed towards the free wall and generates a well-developed symmetrical swirling flow which rotates and moves towards the aortic valve [49]. In DCM, the posterior part of the vortex is far from the wall and decays at a lower speed than in the normal LV. Hence, the loss of symmetry of the transmitral vortex is delayed due to decreased blood-wall interactions in the left ventricle during diastole [47, 63, 64]. 
Therefore, the mitral vortex does not lose its symmetry during diastole and the vortex shape is more spherical and symmetric compared to its elliptical and asymmetric shape in normal subjects [50]. Also in these patients, the blood flow pattern at the vicinity of the aortic valve at the end of diastole is more perpendicular to the LV outflow tract than in normal subjects, which can roll up into vortices. This shortens the pathway of intra-ventricular flow and better orients the flow towards the aorta $[49,65]$. Simulated models of DCM revealed the presence of a delay in vortex detachment from the mitral valve leaflets followed by a reduction in the blood propagation velocity. In addition, a longer stagnation is observed near the apex of a dilated LV which might be a consequence of the left ventricular apical thrombosis formation [66]. Also, in left ventricular dysfunction, the diastolic vortex appears persistent at the middle of the left ventricle during both diastolic and systolic periods and is not dispersed or fully ejected during systole $[45,50]$. Recent studies have shown that not only does the anatomical location and shape of the transmitral swirling pattern vary, but also its power (strength and circulation) is decreased in abnormal LV function such as DCM [45]. The transmitral vortex acts as an energy-conserving structure which stores $16 \pm 8 \%$ of its kinetic energy during the entire diastole phase and converts it to the kinetic energy of the outflow during systole to ensure favourable ejection in a normal LV [67]. In dilated ventricles, the amount of conserved mechanical energy during diastole is reduced (of about 5\%) compare to the normal LV [42, 60]. Examples of the 2D color Doppler blood velocity mapping of normal, dilated, and severely dilated left ventricles in 3-chamber view are shown in Figure 2-4. 


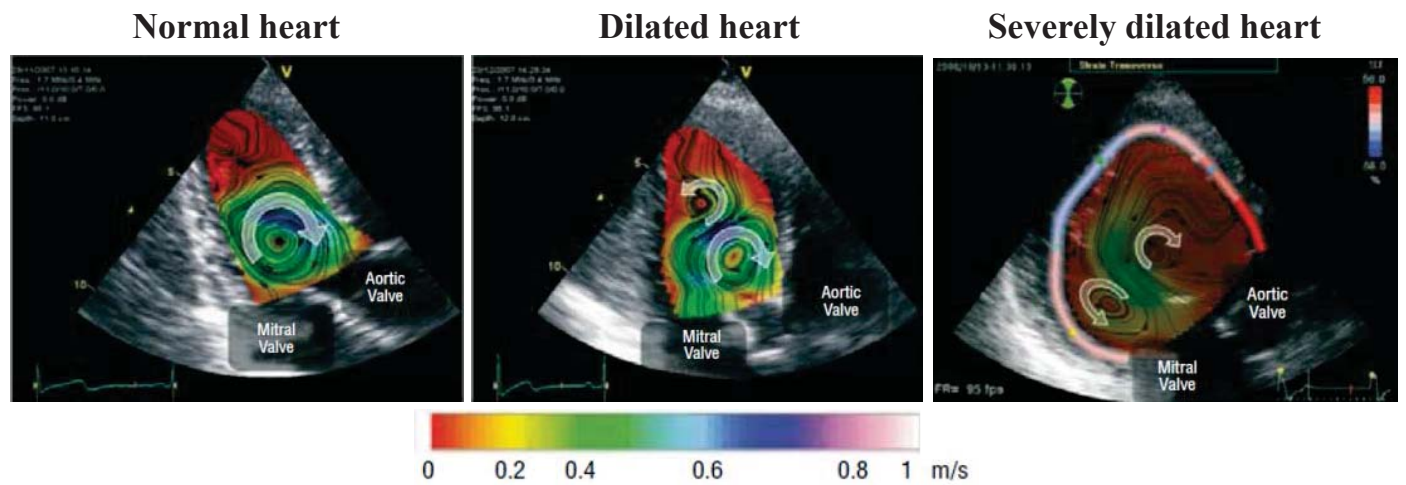

Figure 2-4: 2D color Doppler blood velocity mapping of 3 different left ventricles (normal, dilated and severely dilated subjects) in apical long-axis view. The arrows show the direction of the vortices. Black lines are streamlines and the color shades represent the magnitude of the velocity as shown in the color bar [47].

As it is already mentioned, any structural or functional changes in the left ventricle affect the intra-ventricular flow pattern as well as transmitral vortex formation [52]. Hypertrophic cardiomyopathy $(\mathrm{HCM})$ is another heart muscle disease in which the ventricular walls thicken, especially the intra-ventricular septum. In patients with HCM, The papillary muscles move the entire mitral apparatus towards the LV outflow tract which may reverse the rotational direction of the diastolic vortex and produce an obstruction at the aortic valve as well as disturb the natural propulsion of blood during systole [42, 49, 68, 69].

The natural swirling flow that occurs in a normal left ventricle during LV filling contains only one clockwise asymmetric vortex which is optimized in terms of fluid energy dissipation. In vivo findings also revealed the formation of additional retrograde (counter-rotating with respect to the natural flow circulation) vortices in the presence of cardiac disorders, such as DCM or HCM [63]. Moreover, the size and strength of the aforementioned abnormal apical vortex increases with respect to the progression and severity of the cardiac disease $[47,63]$. Formation of these abnormal vortices is associated with higher fluid energy dissipation, which may result in a significant increase in the physical work of the cardiac muscles and oxygen demand and in turn a reduction in the cardiac pumping efficiency [2, 47, $70,71]$. 


\subsubsection{Mitral and apical vortices: good or bad vortices}

The normal heart cycle is made up of a first pulse (early filling phase considered in this study), a second filling pulse (the atrial contraction which is the extra blood inflow from atrium and accounts for $25 \%$ of ventricular filling), followed by the flow ejection during systolic contraction. Within the left ventricle of a normal heart, the entering flow through the eccentric mitral orifice during early filling develops a large vortical structure behind the longer mitral leaflet and a smaller vortex on the opposite side behind the shorter valvular leaflet [72]. These vortical structures may deform rapidly during filling phase depending on the shape and size of the mitral leaflets. In long-axis 3-chamber view, the larger vortex is easily detectable and the one closer to the smaller leaflet is not immediately visible although it is always present [72]. In this research study, the major circulation pattern close to the longer leaflet is called mitral vortex. The mitral vortex facilitates the blood flow ejection to the left ventricle outflow tract and avoids the fluid stagnation at the cavity apex that could have quite serious consequences such as thrombus formation. Since it contributes to the natural inflow of blood towards the outflow tract, we will also refer to it as the "good" vortex. The small counterrotating vortex (smaller one) that appears adjacent to the posterior mitral valve leaflet is considered as a normal vortex and will not be studied in this research project. 


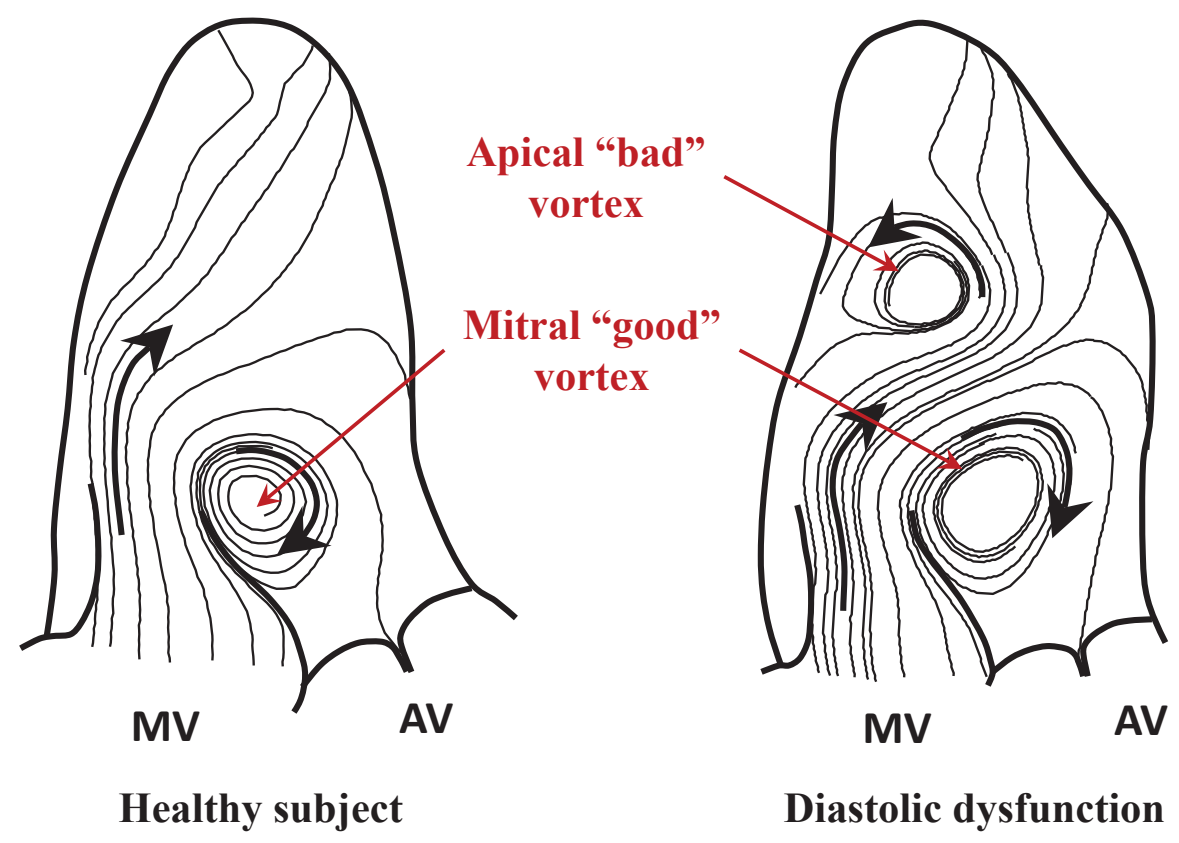

Figure 2-5: Vortex formation in the left ventricle. An apical vortex appears in patients with diastolic dysfunction. MV, mitral valve; AV, aortic valve.

The presence of a single, large and steady mitral vortex during early diastole is a favourable condition in terms of energy conservation, while premature splitting of the vortex into smaller unstable vortices causes excessive impacts and overpressure on the LV walls $[62,73,74]$. In patients with cardiac disease, it seems that the mitral vortex is not alone; an additional vortex may also be present at the apex as clearly shown in Figure 2-5. Due to fluid mass conservation, this apical vortex swirls in the opposite direction to the natural route. Since it adversely affects the natural circulation of intra-ventricular blood, decreasing the ventricular contractile efficiency and dissipating the stored energy, the apical vortex can be termed the "bad" vortex.

\subsubsection{Diagnostic tools for vortex imaging}

Formation of large swirling regions in heart cavities remains a challenging aspect of fluid dynamics. The natural vortices in the LV conserve the momentum of moving blood and prevent sudden flow deceleration due to viscous friction on the walls. In addition, they ensure the timely opening and closing of the mitral valves 
during the cardiac cycle. Through these mechanisms, the intra-ventricular flow fields affect the cardiac pumping efficiency. These swirling vortices that form in the left ventricle during diastolic filling can be determinants and indicators of heart diastolic function. Detection and quantification of such flow structures as well as accurate imaging and understanding of intra-cavitary blood flow patterns are of utmost clinical importance and can guide the selection of appropriate therapy to the patients as fast as possible and stop (or at least slow down) the development of new cardiomyopathies. However, the cardiac diagnosis process has been very challenging since history. Limitations of clinical technology constrain the reliable mapping of the spatial and temporal details of the flow dynamics inside the LV. Physical examination, ECG and chest X-ray are unhelpful for the assessment of intra-ventricular blood flow [75, 76]. Several invasive catheter-derived parameters characterize intrinsic LV diastolic properties accurately, but they cannot be easily used or repeated in daily clinical or research practice [77]. Current available noninvasive non-ionizing flow quantification techniques and tools for both clinical and experimental applications are explained in this section.

\subsubsection{Vortex formation time (VFT)}

Vortex formation time (VFT) is a non-dimensional index that characterizes the optimal conditions leading to vortex formation [78]. VFT is adapted to the LV filling situation and has been shown to be a single index to distinguish different grades of diastolic dysfunction $[42,79]$. VFT is known to be an index of cardiac function which can be easily calculated with a low cost [42]. VFT is defined by length to diameter ratio (L/D) of the fluid column in which $\mathrm{D}$ represents the mitral valve diameter and $\mathrm{L}$ represents the time-averaged inflow velocity through the valve times duration of fluid ejection [79]. VFT also represents a surrogate parameter that is calculated from standard echographic measures such as stroke volume, duration of the $\mathrm{E}$ wave and mitral valve diameter $[79,80]$. It can be calculated by the equation derived by Gharib et al. [79]. 
$V F T=\frac{4(1-\beta) \alpha^{3}}{\pi} \times E F$

In this equation $\beta$ is a fraction of the stroke volume and can be computed from Doppler spectra of the E- and A- (early and late filling) waves. $\alpha$ is a nondimensional index of the LV geometry which can be determined by $\alpha=\frac{E D V^{1 / 3}}{D}$, where EDV is the end diastolic volume in $\mathrm{cm}^{3}$ (or $\mathrm{mL}$ ), and $\mathrm{D}$ is the mitral valve diameter in $\mathrm{cm}$. The mitral diameter can be estimated by obtaining the average of the largest orifice diameter in 2,3, and 4- chamber views.

Considering that $E F=\frac{S V}{E D V}$ and replacing $\alpha$ in the aforementioned equation for VFT results in the following equation:

$V F T=\frac{4(1-\beta)}{\pi D^{3}} \times S V$

$\beta$ in a normal heart at rest is equal to 0.2 [79]. Therefore, the left ventricle VFT under a normal condition can be calculated as VFT $\approx \alpha^{3} . E F$ which is highly dependent on the EF and the LV geometry parameters. For a normal range of EF $(=0.65)$, VFT increases with $\alpha$ that ranges between 1.7 to 2 based on the normal left ventricle EDV and the average mitral valve diameter [79, 81]. During the early phase of diastole the mitral vortex continues to increase its size until VFT reaches an optimal value in the range of $3.3<$ VFT $<5.5$ [79]. This range determines optimal conditions for vortex formation within a normal LV and fully corroborates the VFT values in previous in vitro studies [79, 82, 83]. Out of this range, when the early diastole is completed, the mitral vortex stops growing larger and is pinched off from the starting mitral inflow jet and is also limited in its duration [84].

Previous studies have shown that the vortex formation time index can be affected by certain clinical conditions, such as DCM, placement of artificial heart valves, elevation of afterload and acute myocardial infarction which alter the LV geometry parameter $(\alpha)$ and/ or its function (EF) [79]. In patients with DCM, the 
left ventricle ejection fraction is reduced to 0.3 and results in a significant drop in VFT from its optimal value to the range 1.5 to 2.5 [79]. In patients with mitral valve stenosis, a smaller mitral valve diameter leads to an increase in LV geometry parameter $(\alpha)$ followed by a significant elevation in VFT of up to an order of magnitude $[78,79]$. Recent clinical studies have shown that a brief increase in LV afterload may downshift the VFT as a multifactorial parameter which reflects the LV functional performance in blood transportation processes into the values out of its optimal range $[52,70,80,85,86]$. In patients with high $\mathrm{LV}$ afterload (the required pressure for blood ejection out of the LV during systole), diastolic and systolic function are impaired and also the flow pattern and intra-ventricular vortices within the LV during diastole is altered followed by a decrease in cardiac output and transmitral flow efficiency $[70,87,88]$. VFT has shown a good relation with myocardial infarction size indicating the degree of diastolic dysfunction with the progression of the myocardial damage. Acute myocardial infarcted patients with large infarct size have significantly lower VFT than the optimal value [80]. Recent clinical studies in acute cardiomyopathy have shown that vortex formation time is reduced with impaired relaxation $[52,70,80,85,86]$.

Whether the VFT as a dimensionless, non-invasive index to characterize the efficiency of intra-cardiac blood transportation is clinically of great interest is still questionable. In addition, the sensitivity of this index which is calculated based on the stroke volume on diameter cubed ratio, to error measurements is unknown.

\subsubsection{Phased contrast magnetic resonance imaging (PCMRI)}

Phase contrast magnetic resonance imaging (PCMRI) is the gold standard for direct quantitative blood flow assessment [89-91]. A thorough study of cardiac flow in both normal and abnormal conditions can be performed through PCMRI $[24,25,65]$. This technique has been used for over 10 years to provide timeresolved 3D blood velocity maps in curved or branched blood vessels and also in heart cavities. However, because of the cost and complexity of this technique, a comprehensive clinical characterization of intra-ventricular flow dynamics in 
different levels of cardiac disease has not yet been performed. PCMRI is combined with ECG and respiratory gating to obtain better quality images with higher spatial and temporal resolutions for full intra-cardiac flow mapping in several heart beats $[24,25,49,65,92-94]$. In addition, the acquisition time using PCMRI is dependent on different parameters: size and location of the interested region, the spatial and temporal resolution and respiration pattern [24]. Moreover, PCMRI is time-consuming and cannot be performed safely on patients with implanted pacemakers. [47, 49, 63, 93, 95]

A typical example of pathlines through the left ventricle at early diastole inflow starting from the mitral valve position by means of phase contrast magnetic resonance imaging is shown in Figure 2-6.

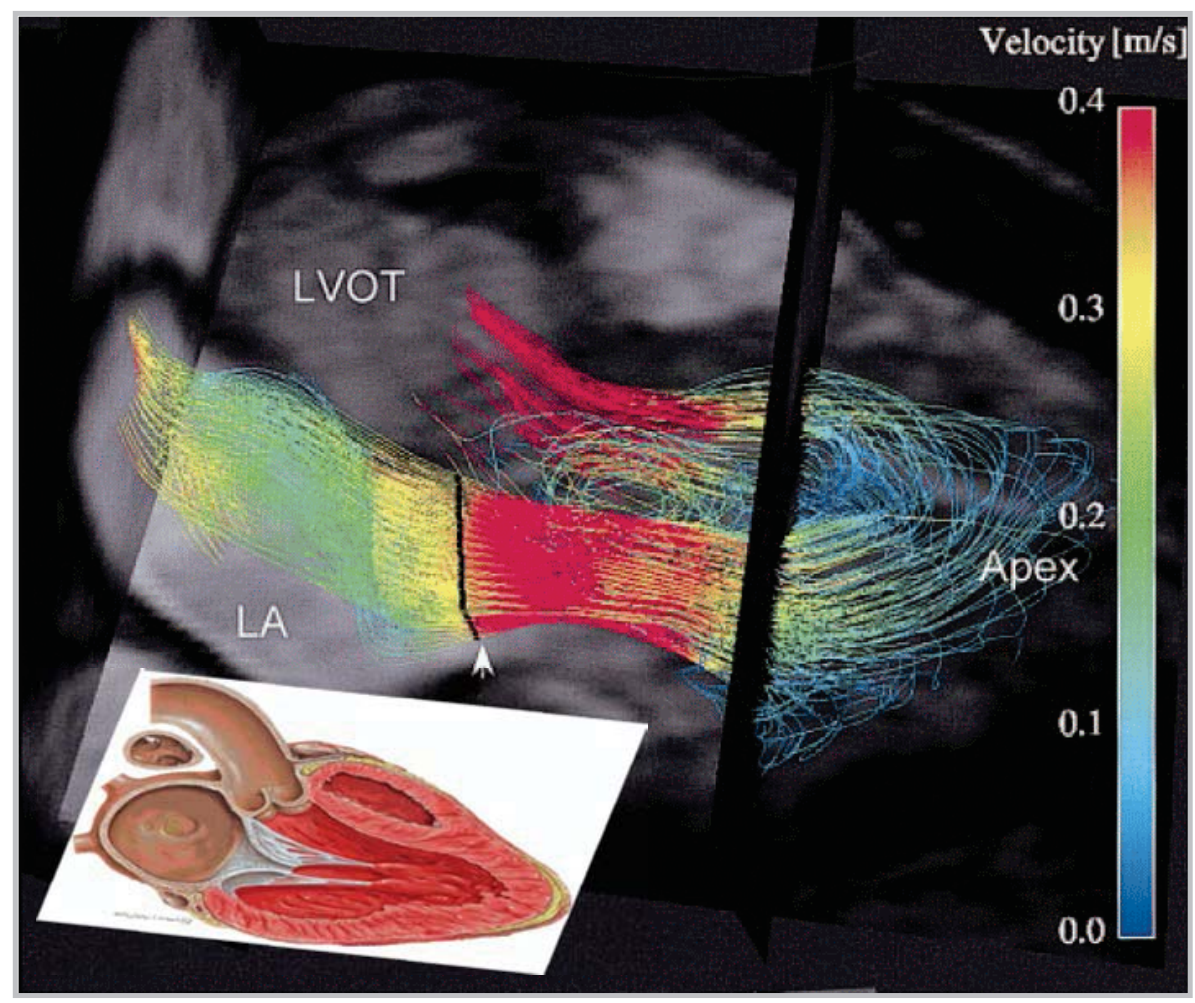

Figure 2-6: Phase contrast MRI at the time of early diastole inflow in a normal heart. In a 2D plane, blood moves forward into the left ventricle and when it reaches the apex it makes a smooth turn into the outflow tract [93]. 


\subsubsection{Color Doppler echocardiography}

Color-Doppler cannot detect velocity components orthogonal to the direction of the scan-lines. The data is therefore a 1D component representation of a $2 \mathrm{D}$ flow and it provides insufficient information for proper quantification of blood dynamic. For example, acquisitions of the carotid artery must be taken at the surface of the neck, almost perpendicular to the vessel; therefore the majority of the velocity information goes undetected and the Doppler angle remains unknown. Angle-dependency of intra-ventricular ultrasound imaging also constrains the assessment of diastolic filling and spatial characterization of flow patterns within the ventricle [70].

\subsection{Crossed-beam vector Doppler ultrasound}

Cross-beam vector Doppler ultrasound [96-99] provides two components of flow velocity from color Doppler ultrasound. The true flow vectors in this method can be obtained by combining Doppler measurements taken at a fixed region of interest from multiple independent directions. This can be performed by means of either multiple probes arranged in different positions or a single probe moved through different positions. The first implementation of crossed-beam vector Doppler technique was proposed by Fox et al. In this tactic, an array of three transducers (two transmitters and one receiver) which focused on the same region of interest with different beam angles, was used to combine the axial and radial velocities in order to estimate true flow vectors [49, 100]. In all crossed-beam vector Doppler techniques, at least two overlapping beams along different directions are required to accurately estimate the velocity vectors. Arigovindan et al. has also proposed a novel technique in which the measurements from different beam directions are combined to reconstruct vector flow from conventional 2D echocardiography. The results for blood flow field in the carotid bifurcation using Arigovindan et al. method is represented in Figure 2-7. The Doppler data acquisitions were performed in three different insonification angles $\left(\alpha=70^{\circ}, 90^{\circ}\right.$, $110^{\circ}$ ) with an ultrasound transducer placed in a fixed position. The visualization of 
the corresponding reconstructed flow field across the vessels before and after the bifurcation is represented in Figure 2-8.
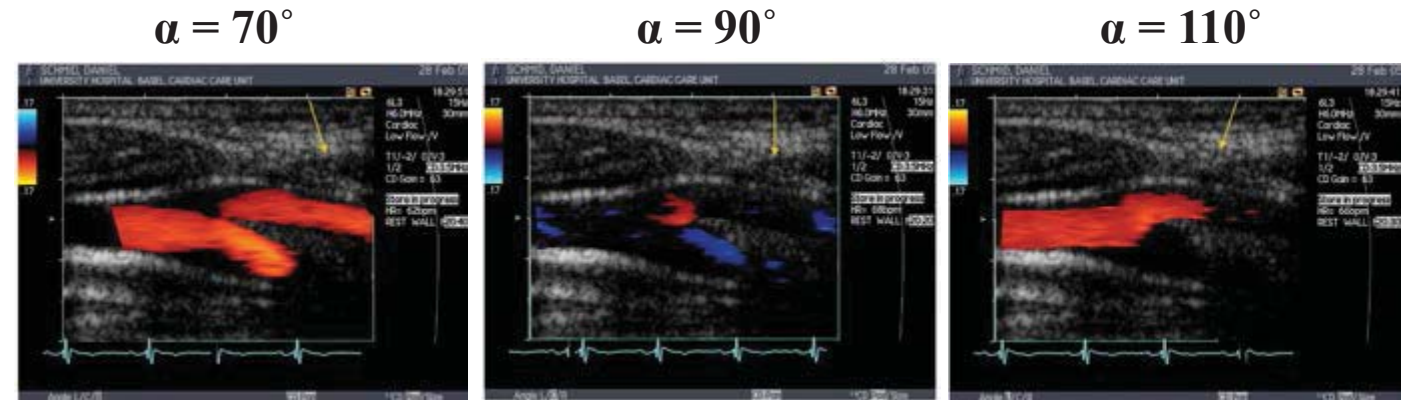

Figure 2-7: Blood flow distribution in carotid bifurcation acquired by Doppler with a fixed probe position in three different beam angles $\left(\alpha=70^{\circ}, 90^{\circ}, 110^{\circ}\right)[101]$.

It is worth mentioning that the cross-beam ultrasound imaging method proposed by Arigovindan et al. works well in vascular applications, [101, 102] but it is not adapted to cardiac imaging as it requires two measurements with a significant angle difference (ideally $>10^{\circ}$ ) in order to obtain the true flow velocity vectors [98].

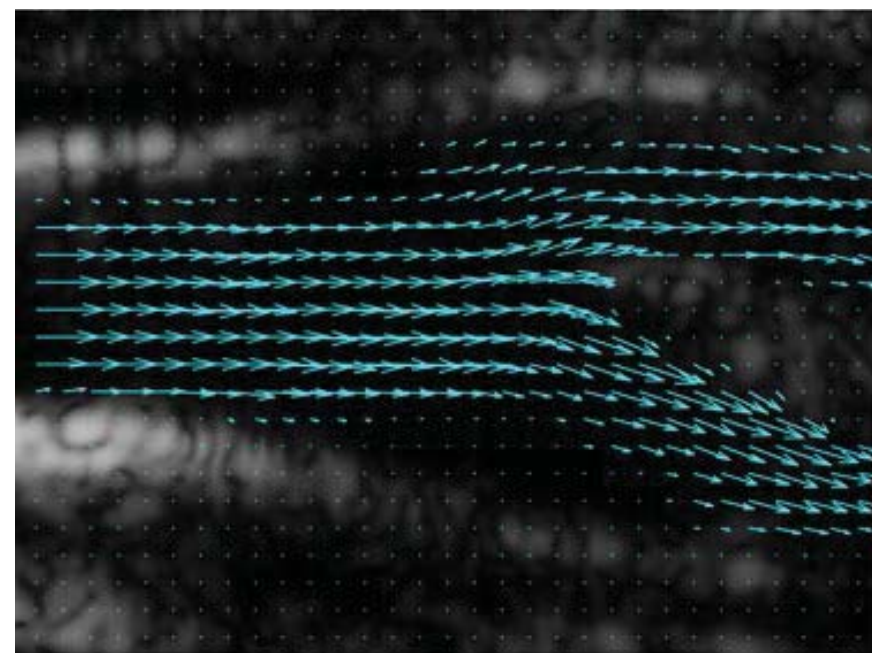

Figure 2-8: Reconstructed flow field in the carotid bifurcation [101]. 


\subsection{Echo particle image velocimetry}

Ultrasound-based PIV, known as echo-PIV [45, 62, 103-108], is another echographic technique for comprehensive intra-ventricular flow visualization and velocity mapping. Combining ultrasound echographic techniques with optical particle image velocimetry (PIV) provides a non-invasive, two dimensional velocity measurements within opaque structures such as blood vessels and heart cavities. Particle image velocimetry $[109,110]$ is a velocity mapping technique utilizing optical imaging in which the flow is seeded with tracer particles that follow the flow dynamics. The particle-seeded flow is illuminated with a light beam generated by a pulsed laser system. Then a camera captures each light pulse in separate image frames. The particles are tracked in different consecutive frames and the displacement data are converted to velocity field by knowing the small time-frame between laser pulses. The PIV technique tracks the patterns produced by groups of seeded particles rather than individual particles [49]. The application of PIV has recently been extended to ultrasound imaging [50]. Echo-PIV (refer to Figure 2-9) technique is based on PIV in two-dimensional B-mode frames obtained by tracking the ultrasound back scattered signal from the injected contrast agent microbubbles in the imaging sequence [48]. The velocity displacement vectors are calculated by splitting the frames into a large number of small rectangular subsections called interrogation areas. The interrogation areas from two consecutive frames are cross correlated with each other, to find the distance that the particle pattern has moved during the time separation between consecutive images. The theory behind cross correlation algorithms is to find an average spatial shift of particles from one interrogation window in one frame to its counterpart in the next frame. It finds the best match (or maximal correlation) for shifted particle patterns in the interrogation windows using statistical methods [48]. To translate that into a velocity measure, this displacement should be divided by the inter-image time [48]. 


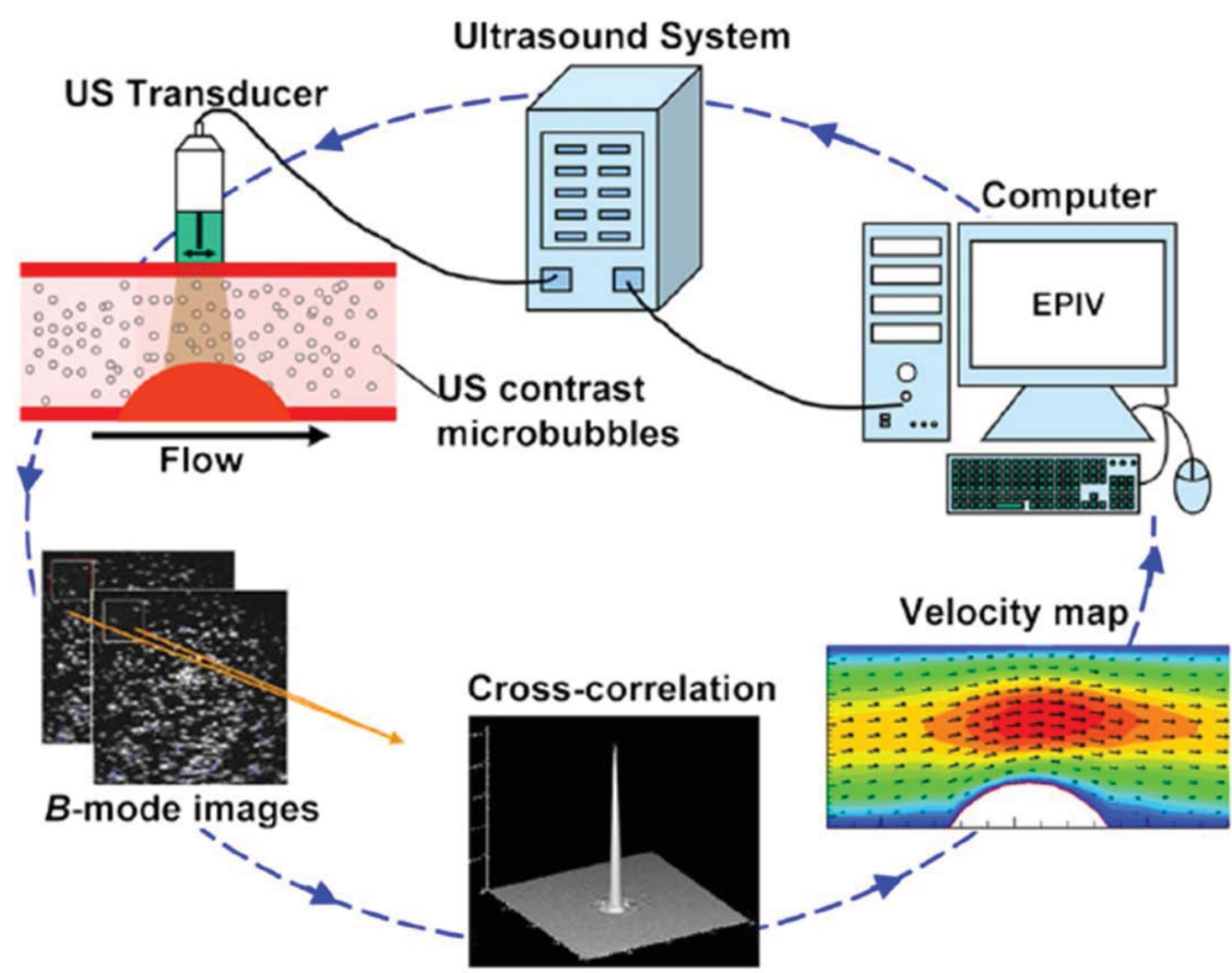

Figure 2-9: Schematic of echo-PIV technique. Contrast agents are seeded into the flow as tracers. Displacement of the particles position and also the velocity vectors can be determined by analyzing two consecutive B-mode images with PIV algorithm. The B-mode images are acquired by ultrasound imaging system [111].

The spatial resolution of echo-PIV acquisition is about five times lower than optical PIV. In addition, its slow image acquisition rate provides a low temporal resolution compared to optical PIV. Although, echo-PIV has the inclination to underestimate velocities greater than a specific range due to its low temporal resolution, it is capable of properly evaluating flow directions, streamlines and swirling vortex flows [50].

Echo-PIV has been validated for the assessment of the ventricular flow by comparison with laboratory-accurate velocity measurements in an experimental left ventricular model $[50,107]$. In addition, its clinical applicability was evaluated in patients with DCM and bioprosthetic mitral valve versus normal subjects [50]. Echo-PIV has recently been used to assess the feasibility of swirling flow analysis 
and to quantify the vortices inside the LV of patients with dilated cardiomyopathy [45]. Echo-PIV has also allowed researchers to evaluate the real time hemodynamic alterations during surgical procedures [112]. In vitro experimentations and in vivo studies involving a small sample size of patient data has showed that 2D echo-PIV is an effective technique for deriving velocity information and can give light for unraveling the underlying physiological flow conditions $[45,107]$.

However, Echo-PIV requires a continuous intravenous injection of contrast agent to reach an image quality compatible with the speckle tracking algorithm [113]. The injected contrast agent (bubbles) must homogeneously fill the heart cavities to prevent saturation and bubble aggregation during flow imaging, especially swirling flow acquisitions [114]. Due to the unstable property of bubble aggregates in the LV, a complex fine tuning of contrast infusion before image acquisition is required which seriously limits the application of echo-PIV in clinical practice [114]. Other limitations of echo-PIV are mostly related to the draw backs of ultrasound imaging technology [49]. For instance, the accuracy of echo-PIV depends on the frame rate of the ultrasound image acquisitions [48] ; the time interval between two consecutive frames must be short for optimal cross correlation [49]. In echo-PIV, the minimum required frame rate to prevent underestimation of the higher velocities is around 60 to 100 frames per second (higher than the heart rate) [49]. However, faster acquisition frame rates, higher than 150 frames per second, for transient flow imaging during isovolumetric contraction phase are necessary [49]. Achieving the highest possible frame rate is strongly dependent on the beam width covering the entire region of interest, imaging depth and spatial and temporal resolution [23]. The desired high frame rate can be obtained by reducing the sector angle [107] as well as the image depth which produces constraints on 2D echo-PIV image acquisition [49]. The spatial resolution obtained by the echo-PIV technique is lower than required [50] and with the limitation on the interrogation window size, the echo-PIV image is confined to a small sector size [63]. The temporal resolution of the echo-PIV 
image seems sufficient for cardiac flow imaging, but it requires important considerations $[48,95]$. Due to the frame rate and the interrogation window size limitations, echo-PIV measures only the confined range of velocities which are lower than $60 \mathrm{~cm} / \mathrm{s}[45,95]$. Recent applications of echo-PIV have shown that for a sector angle of $45^{\circ}$, the highest achievable temporal resolution and spatial resolution are $4 \mathrm{~ms}$ and about $4 \mathrm{~mm}$ respectively [49].

\subsection{Uejima's method}

The vector flow mapping technique, also called echo-dynamography, was developed by Ohtsuki and Tanaka [115] for the measurement of two dimensional blood velocity vectors using conventional color Doppler ultrasound imaging. It assumes that blood flow is composed of a single laminar flow and various rotating flow components. VFM estimates flow vectors in both basic and vortex flows and sums all vectors into a velocity field. In this technique, the velocities perpendicular to the scan plane are assumed to be zero. In the VFM method, the angular velocities are deduced within the entire Doppler field by means of the stream function theory applied to the two dimensional flow. The direction of the base flow vectors can be computed by means of the flow function proposed by the authors. The flow velocity distribution is generated by composing two components of velocity: measured radial and estimated transversal velocities. Figure 2-10 represents an example of flow velocity vectors within the left ventricle of a patient obtained by VFM technique. 


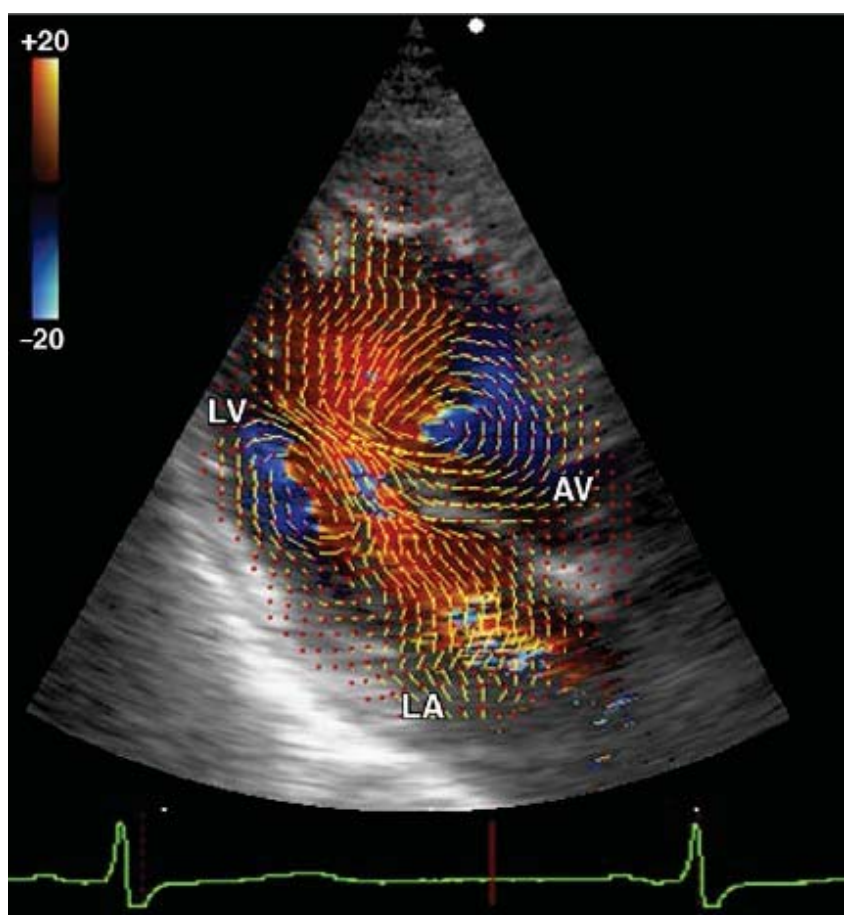

Figure 2-10: An example of vector flow mapping. The flow velocity vectors are superimposed on the color Doppler images. The velocity field at a single point is represented by red dots and the yellow arrows [49]. AV, aortic valve; LA, left atrium; LV, left ventricle.

VFM is based on color Doppler echocardiography and is easy to implement without requiring any injection of contrast agents. VFM is a convenient flow quantification technique for routine clinical applications [95]. Preliminary studies showed promising results regarding the feasibility of LV vortex quantification in patients by VFM [116-120]. The accuracy of velocity estimation with the VFM technique was also tested using a 3D numerical flow model [95].

\subsection{Garcia's method}

A novel technique to construct two dimensional, time-resolved $(2 \mathrm{D}+\mathrm{t})$ velocity fields within the LV using conventional color Doppler flow imaging was recently developed by Garcia et al [63]. It is known that the azimuthal velocities (velocities perpendicular to the scan-line) cannot be calculated with ultrasound devices. However, the radial component of blood velocity can be measured by setting a polar coordinate system centered at the transducer position (cone of the ultrasound 
beam). In this technique the through-plane mass flux in the third dimension is considered to be zero which facilitates the estimation of the cross-beam velocity component from color Doppler ultrasound images. These velocity components at different instances of the heart cycle can be estimated by means of the $2 \mathrm{D}$ continuity equation. The continuity equation simply relates the radial and azimuthal velocity components and can be solved using the boundary conditions. The azimuthal velocity components at the anterior and posterior ventricular walls are determined by means of the B-mode tracking algorithm and are used as the boundary conditions for the continuity equation. The availability of these boundary conditions guarantees the solution of the continuity equation in the polar coordinate. Integrating the continuity equation from both anterior and posterior LV walls separately provides two different estimations for the cross-beam velocity components. Combining the two integrals using an optimization procedure reduces the associated error to the 2D planar simplification in the cross-beam velocity component estimation. The $2 \mathrm{D}+\mathrm{t}$ velocity field can finally be obtained by means of the measured radial velocities and the estimated azimuthal velocities. Such $2 \mathrm{D}+\mathrm{t}$ color Doppler blood velocity maps of the LV apical 3-chamber view can be superimposed on the anatomical B-mode images to facilitate the visual and quantitative assessment of the intra-ventricular flow. The continuity equation is then solved for each ultrasound frame independently which makes the reconstruction algorithm insensitive to the time resolution of the ultrasound image acquisition. The different steps of the numerical process to reconstruct the azimuthal velocities by the Garcia's method are illustrated in Figure 2-11. Also some examples of ventricular blood flow mapping by means of Garcia's method in normal, hypertrophied, dilated subjects are presented in Figure 2-12.

The time resolution of the acquisitions covering the entire LV was approximately 30 frames per cycle with the radial and azimuthal resolutions of $0.5 \mathrm{~mm}$ and 0.02 radians respectively [48]. In order to improve temporal resolution (over 200 frames per cycle), all frames in the cardiac cycle over multiple cycles are decoded 
and merged as a single constructed cycle knowing the offset of each Doppler frame to ECG signal features.

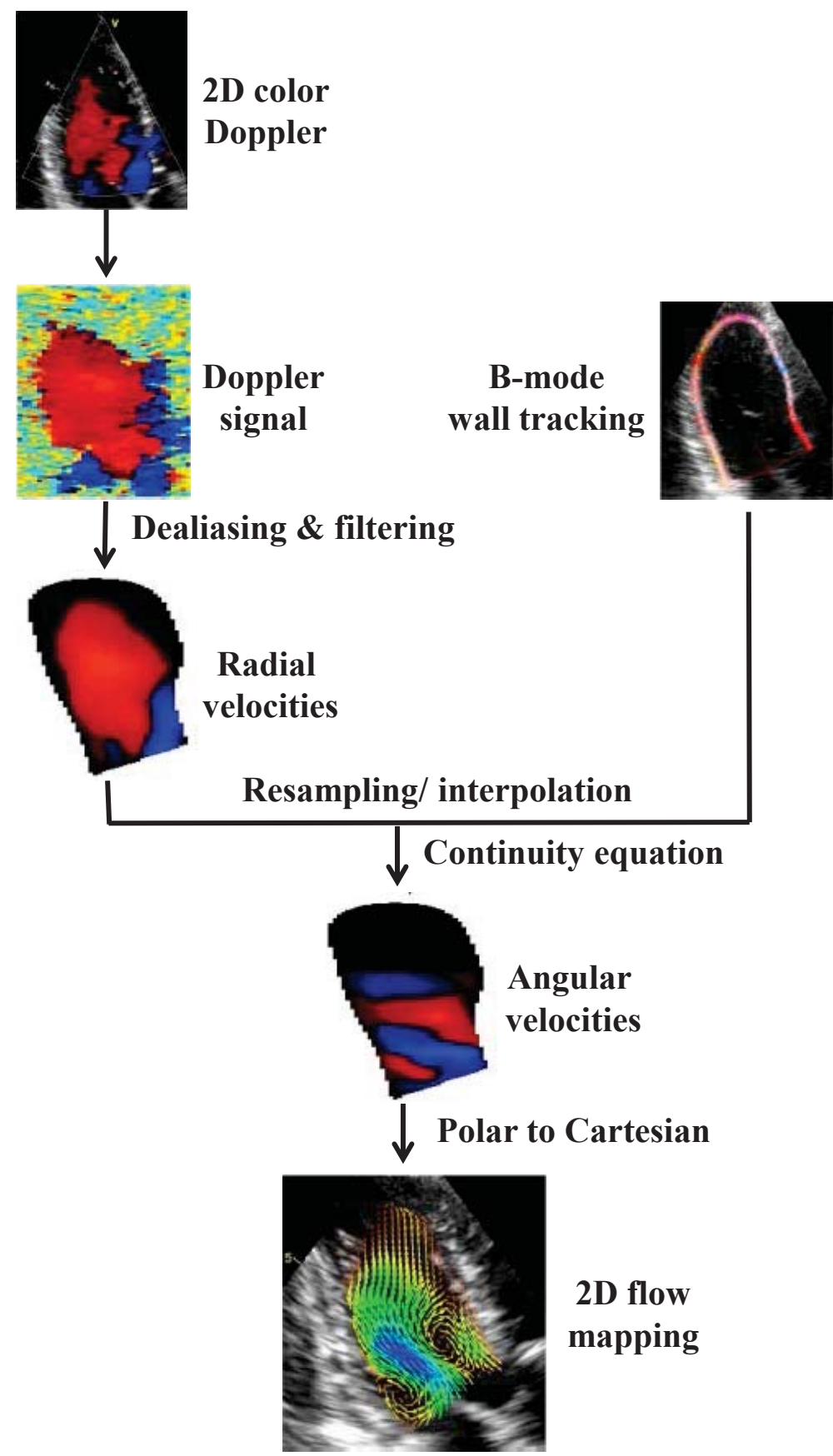

Figure 2-11: Flowchart of Garcia is method for reconstruction of the velocities perpendicular to the scan-line [63]. 
To validate the method, Garcia et al. carried out simultaneously $2 \mathrm{D}+\mathrm{t}$ color Doppler and optical PIV measurements in an atrio-ventricular duplicator under variable hemodynamic conditions [63]. The error due to the planar flow assumption and to scan-plane misalignment was tested in human volunteers including normal subjects and patients with dilated cardiomyopathy using PCMRI. Both in vivo and in vitro measurements revealed that $2 \mathrm{D}+\mathrm{t}$ Doppler flow mapping is a fully non-invasive technique for imaging and quantifying the flow pattern inside the LV during the cardiac cycle based on Doppler-echocardiography. This imaging modality is fast, inexpensive, and does not require any complex training for utilizing in daily clinical practice to assess global LV function directly from $2 \mathrm{D}+\mathrm{t}$ blood velocity measurements. It is based on ultrasound acquisitions that take less than a minute followed by offline processing using standard desktop computers. This technique can be safely performed on patients who have implanted pacemakers and cardiac resynchronization devices who cannot undergo MRI scans [48, 63].
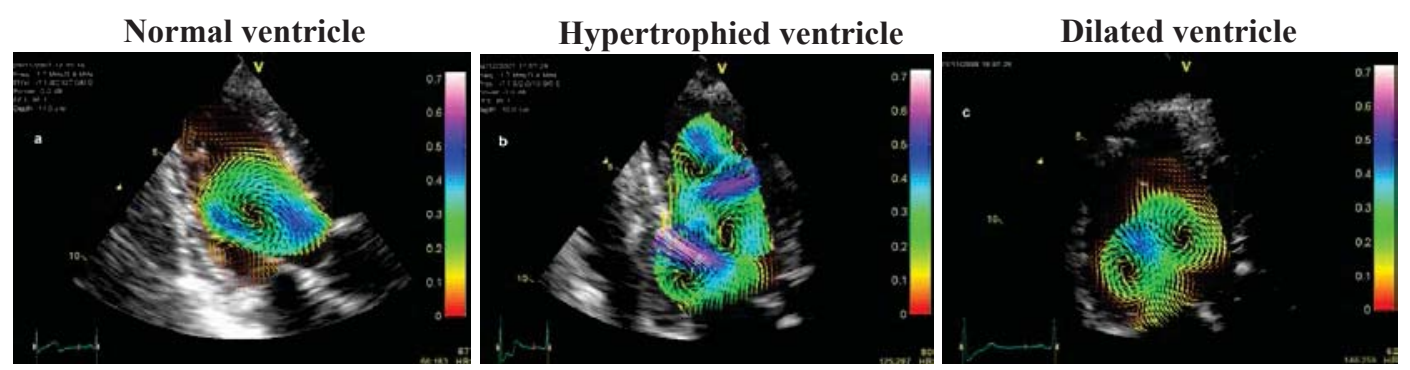

Figure 2-12: Examples of blood velocity mapping on 3 volunteers (normal, hypertrophied, and dilated subjects) in apical long-axis view overlaid on B-mode images. The color shades represents the magnitude of velocities as shown in color bar. The arrows indicate the flow direction [63].

Although this modality provides reasonably accurate validation of the major intraventricular flow patterns suitable for clinical examinations, the B-mode wall tracking algorithm, which provides the essential boundary condition to solve the continuity equation and to obtain the $2 \mathrm{D}+\mathrm{t}$ velocity field, seems to impose some difficulties. 


\subsection{Rationales and hypothesis}

In large vessels (with diameters larger than $1 \mathrm{~mm}$ ) and heart cavities, blood can be assumed as a fluid continuum because its corpuscular nature, in which the fluid is composed of discrete molecules, becomes negligible [42]. As a fluid, and in contrast with solids, blood does not have preferred geometry since it does not possess any elastic behavior. In other words, a solid resists distortion but fluid does not. As a consequence, the flow behavior is highly sensitive to any dynamic and geometric perturbation. The small dynamic and/or geometric modifications may induce a significant change in flow patterns. For instance, in pipe flow, a slight modification in pipe geometry, such as bend or sharpness, may modify the flow patterns drastically. This phenomenon is also true in the left ventricle. In patient with cardiac diseases, the intra-ventricular blood flow pattern is expected to be disturbed due to modification in the blood flow and/ or wall dynamics. We hypothesize that in case of heart disease, the mitral (good) vortex is preserved but with less energy and also an apical (bad) vortex appears which swirls in the opposite direction to the natural flow. Also the presence of the apical vortex may contribute to significant energy dissipation and thus increase the workload. As a result, the myocardial wall is remodeled. This may further disturb the blood flow filling patterns which in turn increases the vorticity of the apical vortex. Based on this, we formulate the following hypotheses which can be tested in a long term study:

1) The presence of an apical vortex in the left ventricle during early filling reflects an impairment of LV relaxation.

2) The presence of an apical vortex is an early marker of diastolic dysfunction, even if the systolic function is preserved.

3) The vorticity and the size of the mitral and apical vortices are related to the degree of the filling impairment. 


\subsection{Goal and objectives}

The long term goal related to this study is to better predict cardiac events in patients with diastolic heart failure by means of intra-ventricular vortex imaging using color Doppler-echo. For this purpose, Dr. Garcia has proposed a new echocardiographic methodology, called Doppler vortography, for a thorough quantification of intra-cardiac vortices based on conventional Doppler images only and developed a complete and easy-to-use software for Doppler vortex imaging in daily clinical practice. The specific objectives of the present project are:

1) To validate the Doppler vortography tool using an in silico model (a computational acoustic model of a numerical vortex field) and

2) To validate the Doppler vortography tool using a state-of-the-art in vitro heart model and optical PIV (not to be confused by echo-PIV!) and

3) To achieve a clinical study in two populations (Normal sedentary subjects and hypertensive patients with normal cardiac phenotype) to test whether this technique is readily applicable in clinical context. 


\section{CHAPTER 3 MATERIALS AND METHODS}

In this section, we describe a new echocardiographic methodology, called Doppler vortography, for a thorough quantification of the vortices that form in the left ventricle during diastolic filling. This technique only requires the use of Doppler velocities obtained by conventional clinical scan; therefore, Doppler vortography is fast, clinically-compliant and does not require any specific adjustments for usual protocol. Assuming that the vortices follow nearly symmetric patterns, the vorticity - which describes the local rotational characteristics of the fluid particles - is estimated at the center of a detected vortex. Since this study focuses only on cardiac imaging using ultrasound, a polar coordinate system $(r, \theta)$ with the origin at the arigo of the phased array transducer is used to delineate the Doppler data. In this configuration, the Doppler velocities depict the radial components of the velocity field.

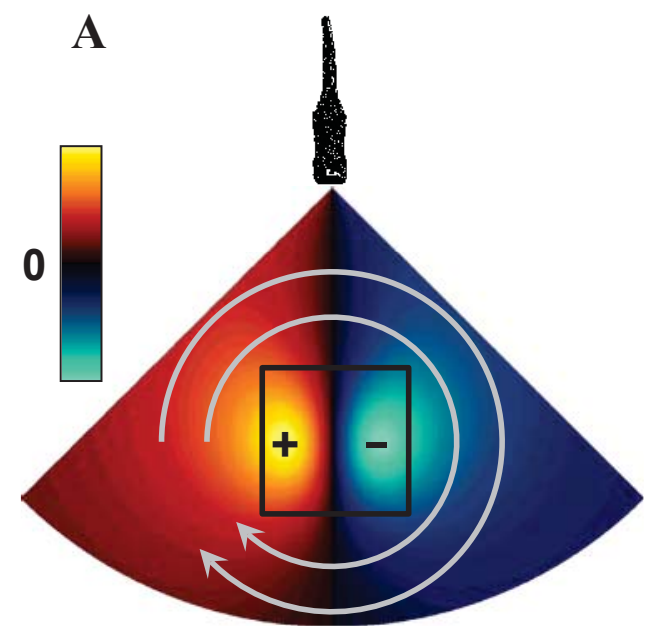

Doppler field

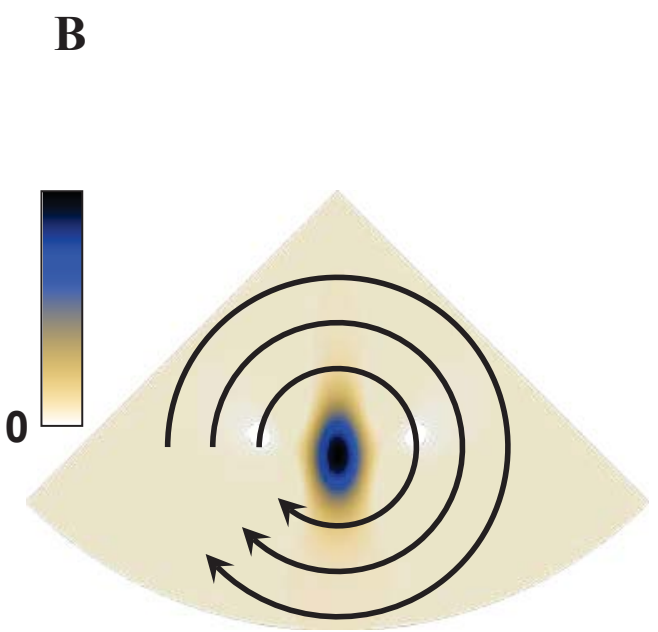

Blood vortex signature

Figure 3-1: A) Single large vortex (Thick arrowed streamlines) and corresponding Doppler field; the phased array transducers at the top. B) Corresponding blood vortex signature (BVS), the BVS amplitude is maximal at the center of the vortex. 
By definition, a vortex as it is explained in section 2.1.1 is a fluid pattern that has a rapid circular or swirling motion around its center. Due to the centrosymmetric properties of a vortex, scanning such a flow pattern using a phased array cardiac probe yields a scanline of zeroes, passing axially through the vortex center, surrounded by two maxima of opposite signs, as portrayed in Figure 3-1.A. Doppler vortography relies on the symmetric properties of the vortices. If $\left(r_{c}, \theta_{c}\right)$ represents the coordinates of the vortex center, the Doppler velocities $V_{D}$ at the vicinity of $\left(r_{c}, \theta_{c}\right)$ can be written as:

$V_{D}\left(r, \theta-\theta_{c}\right) \approx-V_{D}\left(r,-\theta+\theta_{c}\right)$, if $r \approx r_{c}$ and $\theta \approx \theta_{c}$

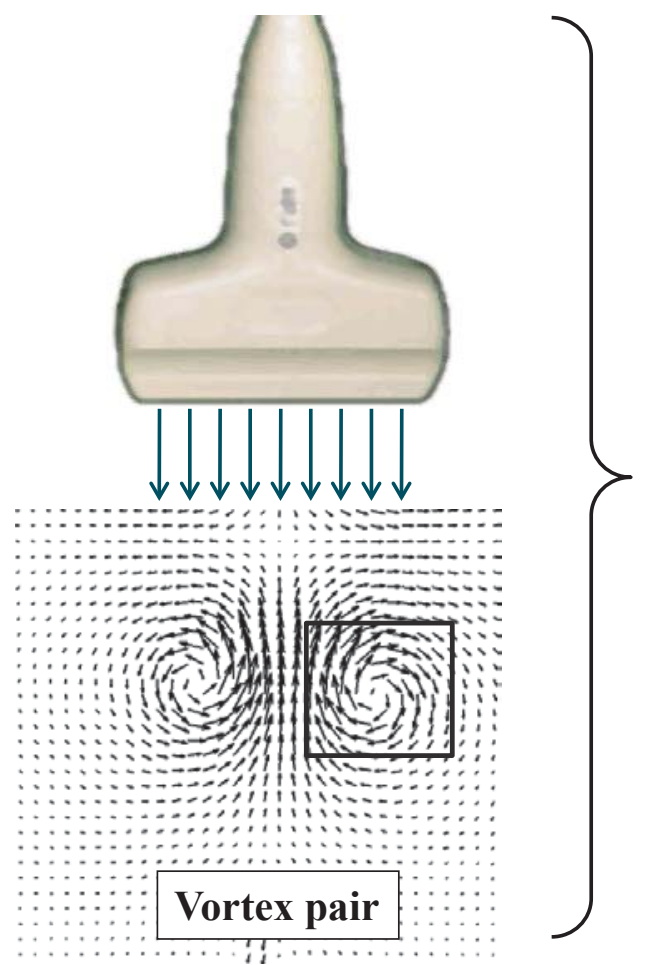

color Doppler

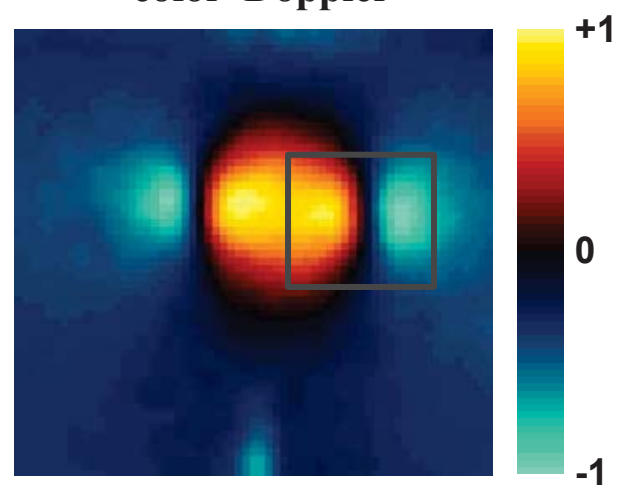

Figure 3-2: Left: a numerical vortex (vortex pair) is scanned by a probe. Right: corresponding Doppler data. At the vortex center the velocity is nearly zero surrounded by two maxima of opposite signs.

Such properties induce specific symmetries in the Doppler field which can be exploited to describe the vortices quantitatively. Using this property, we can define a simple kernel filter that returns a parameter (blood vortex signature (BVS)) that 
reflects the presence of intra-cardiac vortices. For this purpose the Doppler velocities are considered as a matrix array $\left(V_{D_{i j}}\right)$.

The BVS parameter (refer to fig.3.3) can be computed as:

$B V S_{i j}=R\left(\operatorname{cov}\left(W_{i j},-f \operatorname{lip} \operatorname{lr}\left(W_{i j}\right)\right)\right) \times S_{i j}$

Let $W_{i j}$ represents a small $\mathrm{m} \times \mathrm{n}$ matrix centered on the vortex core (refer to Figure 3-1 and Figure 3-2).

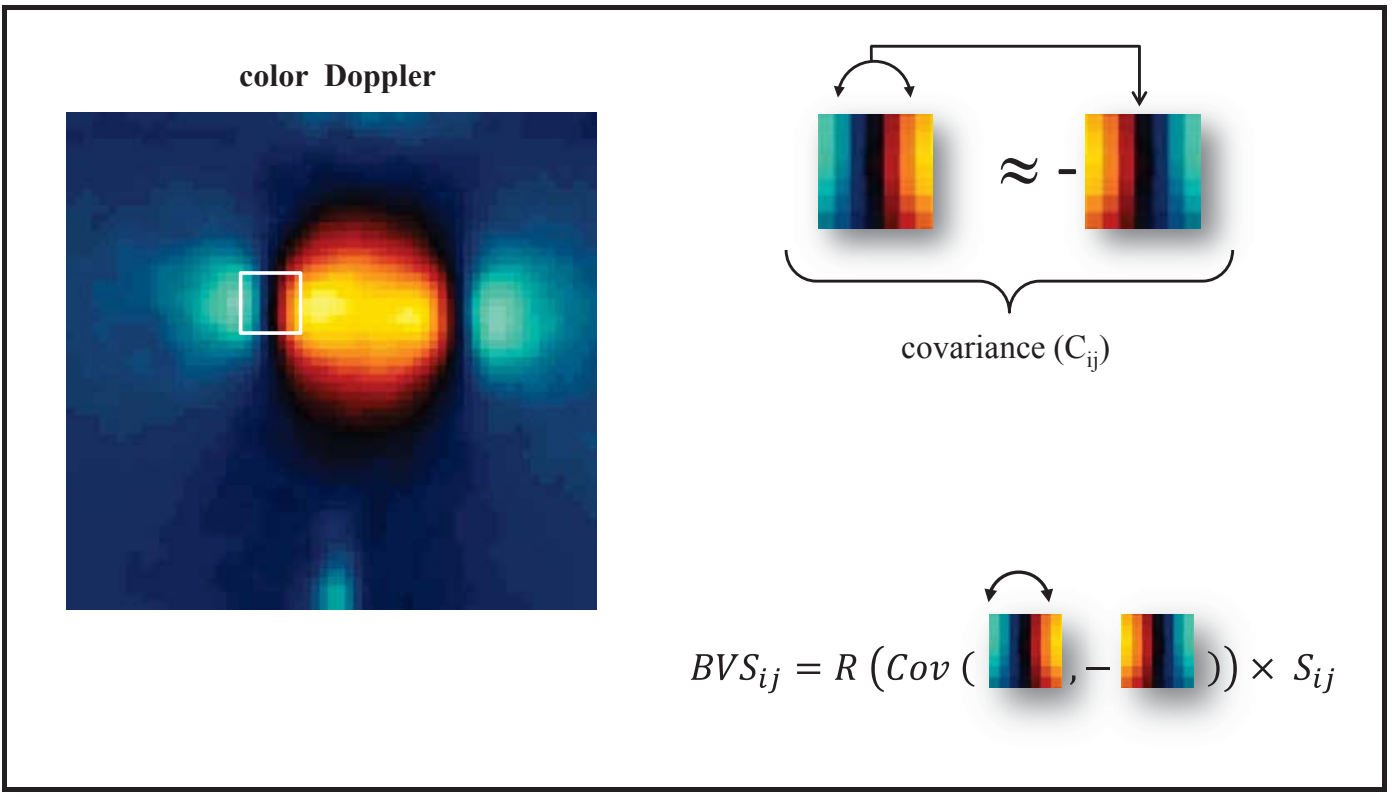

Figure 3-3: Basic concepts of blood vortex signature (BVS). R and S stand for the ramp and sign functions, respectively. Left panel: raw Doppler data of a vortex pair in the Cartesian coordinate system.

The core of the vortex is defined as the radius where the tangential velocity is maximum. As we know from fluid dynamics the velocity at the center of a vortex is nearly zero and this zero scanline is surrounded by the velocity vectors with opposite signs at the vicinity of the vortex core. If we deliberately swap the left and right side of the window $\mathrm{W}$ around, it results in a change in the velocity signs on both sides of the window like taking the mirror image with respect to scanline crossing the vortex center (an angular mirror line). Therefore, flipping left to right of a small window located at the vortex core mostly modifies its sign only. The 
negative of the mirrored matrix $\left(-f \operatorname{lip} \operatorname{lr}\left(W_{i j}\right)\right)$ will be equal to the original matrix $W_{i j}$ at the vortex center. Using this concept that the velocity vectors in the original and the negative mirrored window tend to show similar behavior, the covariance of these two at the vortex core will be positive. Outside the vortex core, if the original and the negative mirrored matrices are independent then their covariance will be nearly zero indicating no correlation. Hence, in order to specify the vortex center we just need to consider the positive covariance between the small window $W_{i j}$ and the negative flip left to right of the window $\left(-f \operatorname{liplr}\left(W_{i j}\right)\right)$ by using the ramp function which is represented by $\mathrm{R}$ in the equation (3-2). Due to the presence of the positive covariance in (2), the blood vortex signature is expected to have high amplitude at the vicinity of the vortex and reaches the local maximum at its center. To find the direction of the vortex (clockwise and counter clockwise), we need to sum up the differences between the columns of the original window $W_{i j}$ and consider just the sign of the product. The positive or negative results prove having a clockwise or counter-clockwise vortex respectively. The $S_{i j}$ in (2) reflects the direction of the vortex. As an example, fig.3.1.B represents the BVS map related to the Doppler field and it is noticeable that the BVS amplitude is maximal at the center of the vortex.

Once the location of the vortex center has been determined, the strength of the vortex should be defined. A vortex can be mainly described by its core vorticity (refer to section 2.1.1), which is the local rotation rate of the fluid particles at the center of the vortex. The vorticity measures the angular velocity of the fluid particles by computing the vector product of $\nabla$ and velocity vectors (the curl of the velocity field), $\nabla \times V(r, \theta)$. It is a vector quantity having two scalar components. In a two dimensional polar coordinate system, the vorticity about the $\mathrm{z}$ axis, perpendicular to the plane of interest, can be calculated by equation (3-3) [121].

$\omega=\frac{1}{r}\left(\frac{\partial}{\partial r}\left(r V_{\theta}\right)-\frac{\partial V_{r}}{\partial \theta}\right)$ 
where $V_{r}$ and $V_{\theta}$ are the polar and angular velocity components, respectively. Considering that the Doppler echocardiography provides only the radial component of the velocity $\left(V_{r}=-V_{D}\right)$, the above equation requires some modifications in order to use it in clinical context. Assuming that the vortices follow a nearly axisymmetric pattern at the vicinity of its core (i.e. we assume that the vortex forms a perfect disk close to its center), one gets at the center of the $\operatorname{vortex}\left(r_{c}, \theta_{c}\right)$ :

$\frac{\partial}{\partial r}\left(r V_{\theta}\right)=-\frac{\partial V_{r}}{\partial \theta}$, for $(r, \theta)=\left(r_{c}, \theta_{c}\right)$

Applying equation (3-4) to the general vorticity equation (3-3), the core vorticity $\omega_{c}$ can finally be derived as a function of the Doppler velocities:

$\omega_{c}=-\left.\frac{2}{r_{c}} \frac{\partial V_{r}}{\partial \theta}\right|_{r_{c}, \theta_{c}}=\left.\frac{2}{r_{c}} \frac{\partial V_{D}}{\partial \theta}\right|_{r_{c}, \theta_{c}}$

To sum up (refer to Figure 3-4), Doppler vortography works as follows:

1) The "blood vortex signature" (BVS) is measured during early filling from the color-Doppler data using Eq. (3-2).

2) The main vortices are detected by seeking the extrema of BVS.

3) Their core vorticities are estimated using Eq. (3-5). 


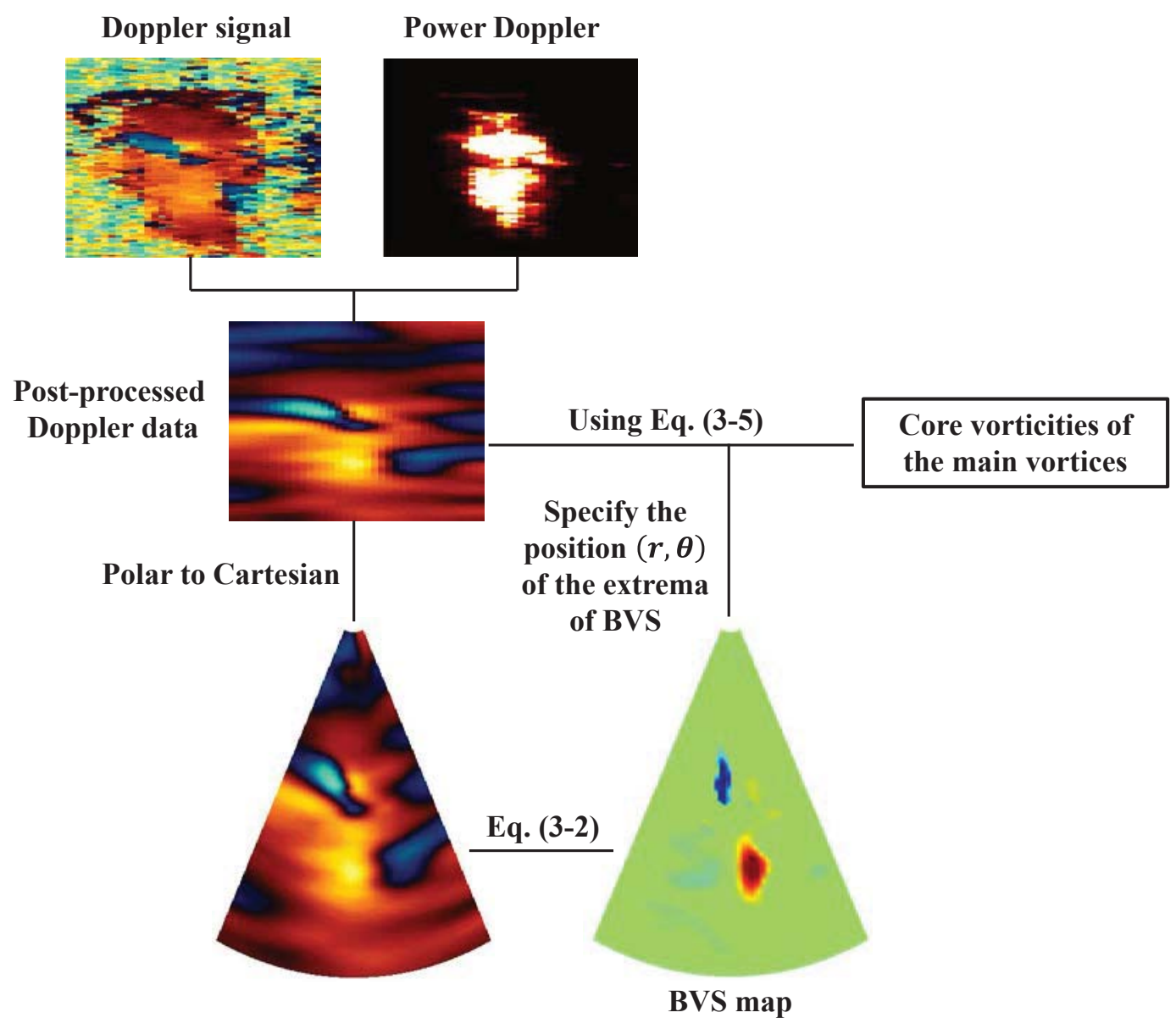

Figure 3-4: Flowchart of Doppler vortography method for specifying the main vortices and calculating their core vorticities (vortography-derived vorticities) in the left ventricle during diastole phase.

Doppler vortography is exemplified by a numerical vortex model illustrated in Figure 3-5. As it can be seen, a vortex pair is scanned with a cardiac probe (Figure 3-5A). The corresponding Doppler velocity field is obtained by considering only the velocities along the scan-line (Figure 3-5B).

In addition, a control method is also required to compare the simulated velocities (vortography-derived velocity). The control method called 8 point method [122] utilizes the original two dimensional velocity fields to calculate the core vorticity and represent it as the vorticity map (Figure 3-5C). In vector calculus, the vorticity $\omega$ calculated from the velocity field is related to the circulation $\Gamma$ by Stokes' 
integral theorem. So, the circulation is defined as a line integral evaluated along the velocity component that is locally tangent to the contour.

$\Gamma=\oint U \cdot d l=\int(\nabla \times U) \cdot d S=\int \omega \cdot d s$

In this equation $l$ is the path of integration around a closed surface area $S$ in a fluid. The Stokes theorem can also be applied on PIV velocity measurements on a Cartesian axes mode.

$\left(\bar{\omega}_{z}\right)_{i, j}=\frac{1}{A} \Gamma_{i, j}=\frac{1}{A} \oint_{l(X, Y)}(U, V) \cdot d l$

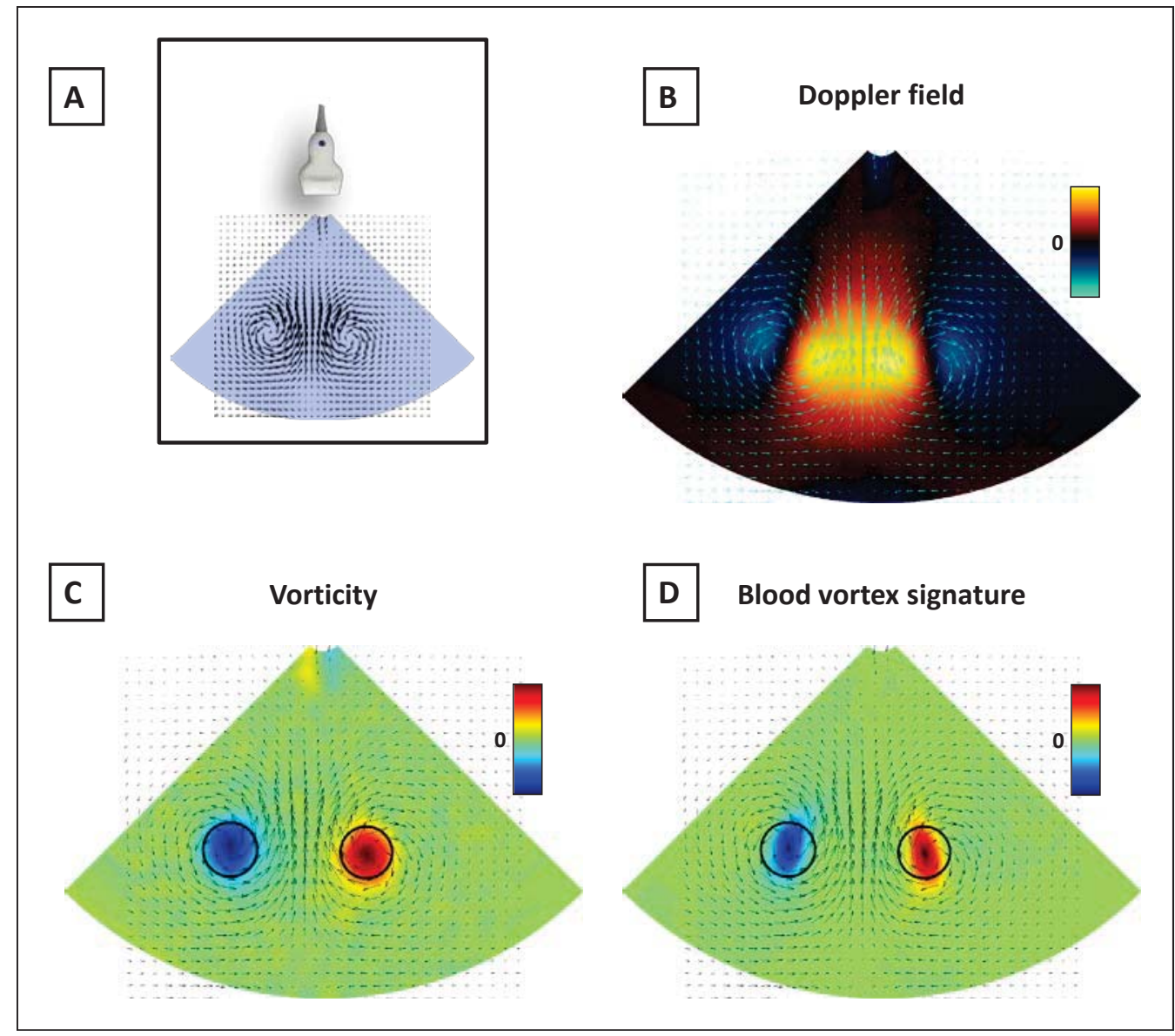

Figure 3-5: A) Numerical model: a vortex pair is scanned with a cardiac probe. B) Corresponding Doppler velocities (without additive noise). C) Vorticity map (reference) calculated from the original vector flow field. D) BVS map related to the Doppler field in B. 
Where $\left(\bar{\omega}_{z}\right)_{i, j}$ is the average vorticity in an enclosed area. The 8-point vorticity calculation method determines the vorticity by specifying a small rectangular contour around the position where the circulation calculation will be done (refer to Figure 3-6).

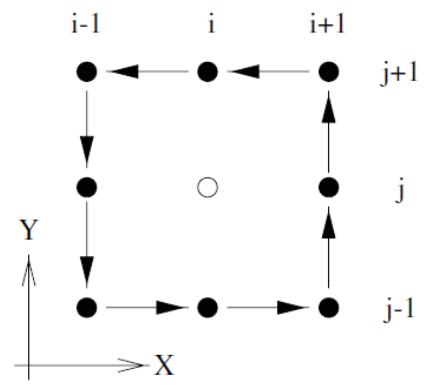

Figure 3-6: The rectangular contour used to calculate circulation and estimate the vorticity at point $(\mathrm{i}, \mathrm{j})[122]$.

The circulation can be computed around the neighbouring eight points by utilizing a standard integration strategy such as trapezoidal rule. To obtain the average vorticity, the local circulation has to be divided by the enclosed area.

$\left(\bar{\omega}_{z}\right)_{i, j} \cong \frac{\Gamma_{i, j}}{4 \Delta X \Delta Y}$

With

$$
\begin{aligned}
\Gamma_{i, j}=\frac{1}{2} \Delta X\left(U_{i-1, j-1}+2 U_{i, j-1}+U_{i+1, j-1}\right) \\
+\frac{1}{2} \Delta Y\left(V_{i+1, j-1}+2 V_{i+1, j}+V_{i+1, j+1}\right) \\
-\frac{1}{2} \Delta X\left(U_{i+1, j+1}+2 U_{i, j+1}+U_{i-1, j+1}\right)-\frac{1}{2} \Delta Y\left(V_{i-1, j+1}\right. \\
+ \\
\left.+2 V_{i-1, j}+V_{i-1, j-1}\right)
\end{aligned}
$$


The vorticity map calculated from the original vector flow field and the BVS map related to the Doppler field is illustrated in Figure 3-5C and D respectively.

The reliability of the BVS measured by Doppler vortography was assessed in mock Doppler fields issued from numerical simulations (in silico) and in vitro data. Doppler vortography was also tested in 18 patients with cardiac diseases.

\subsubsection{In silico: ultrasound imaging and simulations}

A computational acoustic model was first used to produce mock Doppler fields to test the accuracy of Doppler vortography under ideal conditions. The Field II software developed by Jensen and Svendsen [123, 124] was employed to simulate the raw ultrasound signals resulting from a numerical vortex flow. This simulation software allows modeling of ultrasound transducers and practical image acquisition sequence.

This numerical ultrasound simulation was performed with a Lamb-Oseen vortex. The Lamb-Oseen vortex is a classical time decaying vortex which is commonly used in fluid dynamics. The mathematical model for the Lamb-Oseen velocity field in the circumferential direction using the peak tangential velocity $V_{\theta_{\max }}$ of the vortex is described by [125]:

$V_{\theta}(r)=V_{\theta_{\max }}\left(1+\frac{0.5}{\alpha}\right) \frac{r_{c}}{r}\left[1-\exp \left(-\alpha \frac{r^{2}}{r_{c}{ }^{2}}\right)\right]$

Where $\alpha \approx 1.25643, r$ is the radius, and $r_{C}$ is the core radius of the vortex.

In this part, the blood flow is simulated by propagating the randomly distributed point scatterers. The positions of the moving particles were updated between each ultrasound frame. A two dimensional 64 elements phased array transducer having a center frequency of $2.5 \mathrm{MHZ}$, an azimuthal pitch of $0.3 \mathrm{~mm}$ was used in the in silico measurements. The sampling frequency of the raw ultrasound signals was set to $100 \mathrm{MHZ}$ during simulation. A total of 40,000 randomly positioned scatterers were simulated and insonified with pulses containing 8 cycles. The region of interest was $4 \mathrm{~cm}$-wide and ranged from 4 to $8 \mathrm{~cm}$ depth respective to the 
probe. The centers of the Lamb-Oseen vortices were all positioned within this region of interest. For a given vortex configuration, five RF images containing 50 scanlines and covering a $40^{\circ}$ wide sector were created. The consecutive RF images were generated after the scatterers had been displaced according to the LambOseen velocity field. A complex base-band modulation technique called IQdemodulation (refer to section 1.3.3.7) was performed to reduce the amount of data without losing any essential information. The Doppler velocity field, which are the velocities in the radial direction, was measured using the autocorrelation algorithm described by Kasai et al. [126]. These five RF images have been used in the auto-correlation technique to provide one Doppler signal. The waiting time between each frame acquisition was always given by the maximum available pulse repetition frequency $(3 \times \mathrm{PRF})$ regarding the peak tangential velocities for different flow fields, so that aliasing was avoided.

The simulation protocol was done for 31 vortices with different radii and peak tangential velocities. To generate these scenarios, the vortex core radius $\left(r_{c}\right)$ was fixed at $1,1.25$ and $1.5 \mathrm{~cm}$ and the peak tangential velocity $\left(V_{\theta_{\max }}\right)$ was ranged from 0.35 to $1.5 \mathrm{~m} / \mathrm{s}$. These radii and velocities were chosen to be in a physiological range for the vorticity in the left ventricle $\left(\sim 90-320 \mathrm{~s}^{-1}\right)$ [63].

The Doppler fields were smoothed using an unsupervised smoothing and denoising method $[127,128]$. The BVS was calculated in all these velocity fields using equation (3-2). Also the local extrema of the BVS patches were detected and the core vorticities were estimated using equation (3-5). The consecutive steps of the numerical simulation process are illustrated in Figure 3-7. 


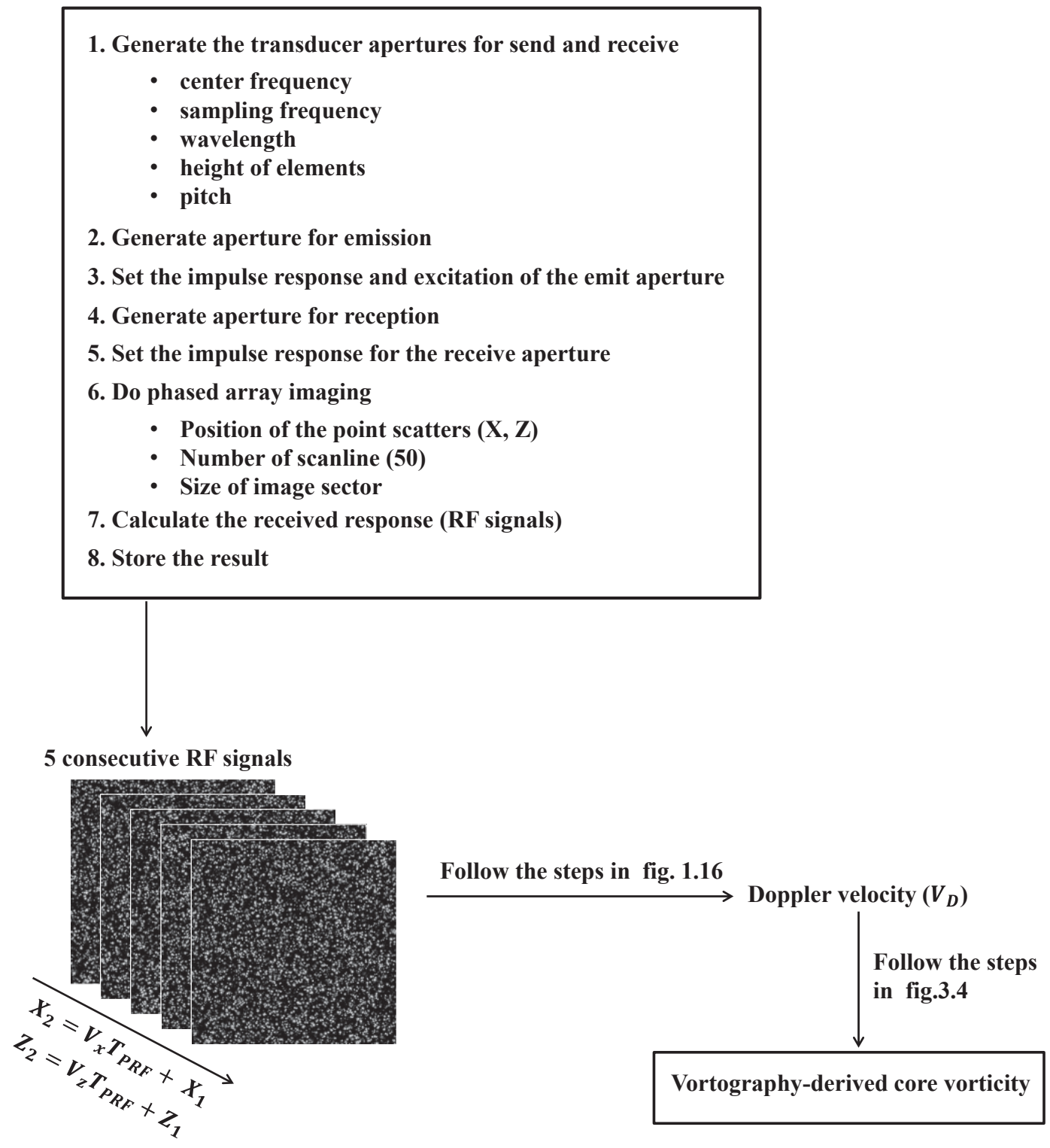

Figure 3-7: Flowchart of in silico simulation using Field II software. The scatterers were displaced according to the Lamb-Oseen vortex $\left(V_{x}, V_{z}\right)$ which was performed between each image acquisition. The time between each image acquisition $\left(T_{P R F}\right)$ was adjusted so that aliasing was avoided. $(\mathrm{X} 1, \mathrm{Z} 1)$ and $(\mathrm{X} 2, \mathrm{Z} 2)$ are position of the point scatterers before and after displacement respectively. 
Several kernel sizes (i.e. $(2 n+1) \times(2 n+1)$ with $n=1 \ldots 5)$ were tested to analyze the robustness of the method. The effect of the kernel size was analyzed using a repeated measures analysis of variances by means of an easy-to-use statistical software called MedCalc (version 12.6).

Knowing the velocities in the $\theta$ direction of a vortex combined with the general vorticity equation (3-3), the ground-truth vorticity at the center of the Lamb-Oseen vortex $(\mathrm{r}=0)$ can be found as:

$\omega_{c}=\frac{-2 \times V_{\theta_{\max }} \times(\alpha+0.5)}{r_{c}}$

The Doppler-derived core vorticities (Eq. 3-5) were compared with those measured from an original vector flow field (ground-truth vorticities) (Eq. 3-11) using a linear regression and a Bland-Altman plot.

\subsubsection{In vitro analysis based on PIV experimentation in a left heart model}

The Doppler fields simulated with Field II were all based from perfectly axisymmetric vortices. Therefore, Doppler vortography method was also performed with more realistic vortical patterns issued from a physiological heart model using optical Particle Image Velocimetry (PIV). In vitro experiments were performed using an atrioventricular dual activation pulse duplicator (ESIL, Marseilles, France). As previously described in [129], the in vitro setup is composed of ventricular and atrial activation boxes, systemic and pulmonary circulation model and a computerized driving interface. Figure 3-8 represents an overview of the left heart model setup. As it can be seen, it is composed of 1) the three main components PIV configuration (including the laser, the micrometric displacement system, and the cameras), and also 2) the mock circulatory system (pulmonary veins, the left atrial and ventricular chamber, and the systemic model). 

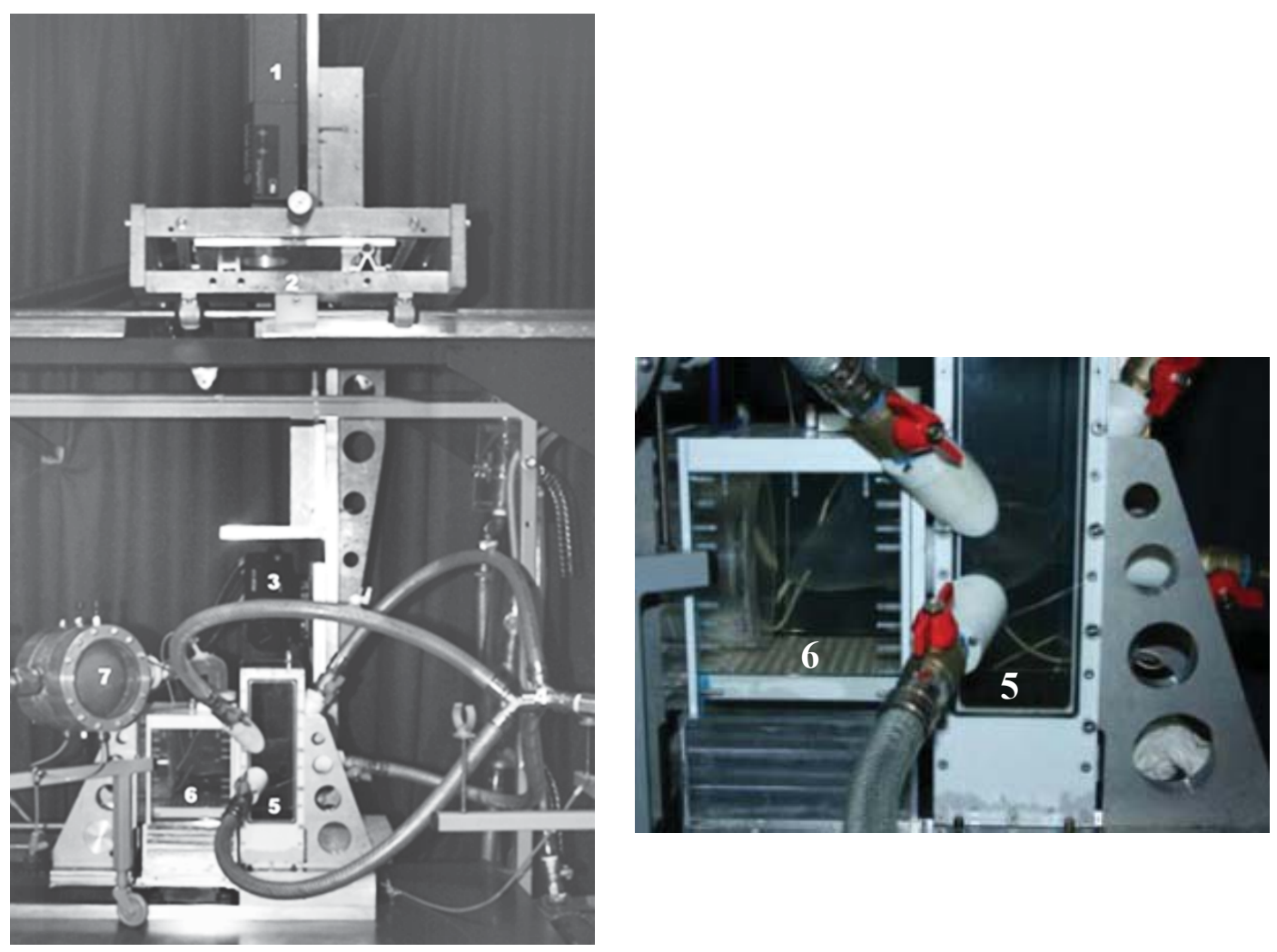

Figure 3-8: Left panel: overview of the in vitro setup [130]. The different components are (1) laser, (2) micrometric displacement system, (3) camera, (4) pulmonary veins, (5) left atrial, (6) left ventricle, and (7) systemic model. Right panel: zoom the left atrio-ventricular device (parts (5) and (6)) [129].

The left heart cavities are compliant and transparent to reproduce the contraction and the relaxation of cardiac cavities and also to assess the velocity fields inside the LV using optical PIV. To simulate ventricle dynamics (contraction or relaxation), a gear pump transfers the extra-circulatory fluid from an open tank to a box containing the ventricle model. During the systole period, while the fluid is injected to the space between the ventricle and the surrounding box, it compresses the ventricle and ejects the intra-circulatory fluid contained in the ventricle into the aorta. During diastole period, the pump sucks the extra-circulatory fluid from the box, the ventricle dilates, and the intra-circulatory fluid contained in the atrium passes through the mitral valve and enters the ventricle. The same principle is used for the atrium to simulate the contraction of the atria and to support the active ventricular filling. 
In order to eliminate any optical distortion, the same fluid is used in the in vitro system for both in and outside of the ventricle. A liquid made of $40 \%$ glycerol and of $60 \%$ of saline water was used to reproduce blood viscosity. The circulatory fluid was seeded by neutrally-buoyant Nylon particles with a mean diameter of 15-20 $\mu \mathrm{m}$. The time between two frame exposures was set to $\Delta T=40 \pm 3(\mathrm{~ms})$ as a compromise between the intraventricular velocities and the available power of the laser.

Three different hemodynamic conditions were simulated in the in vitro system: heart rate $(\mathrm{bpm}) /$ stroke volume $(\mathrm{mL})$ equals to $60 / 65,80 / 60$ and 100/75. The PIV laser plane traversed the mitral valve, aortic valve and ventricular apex to simulate an apical long-axis view. Velocity maps were generated with a standard commercial software package (Insight3G, TSI Inc., Shoreview, MN) using an interrogation size of 200 pixel $\times 64$ pixel. The average velocity fields were obtained over 30 consecutive cardiac cycles.

Using the original velocity fields provided by PIV, the mock Doppler velocity fields were simulated with the transducer located in the apical position. As performed with the numerical simulations, the BVS was calculated using equation (3-2) with different kernel sizes after a prior post-processing of the noisy reconstructed Doppler velocity field. The local extremum of the BVS patch corresponding to the main diastolic vortex was detected and the core vorticity was estimated by means of equation (3-5). The consecutive steps of the in vitro process are illustrated in Figure 3-9. 

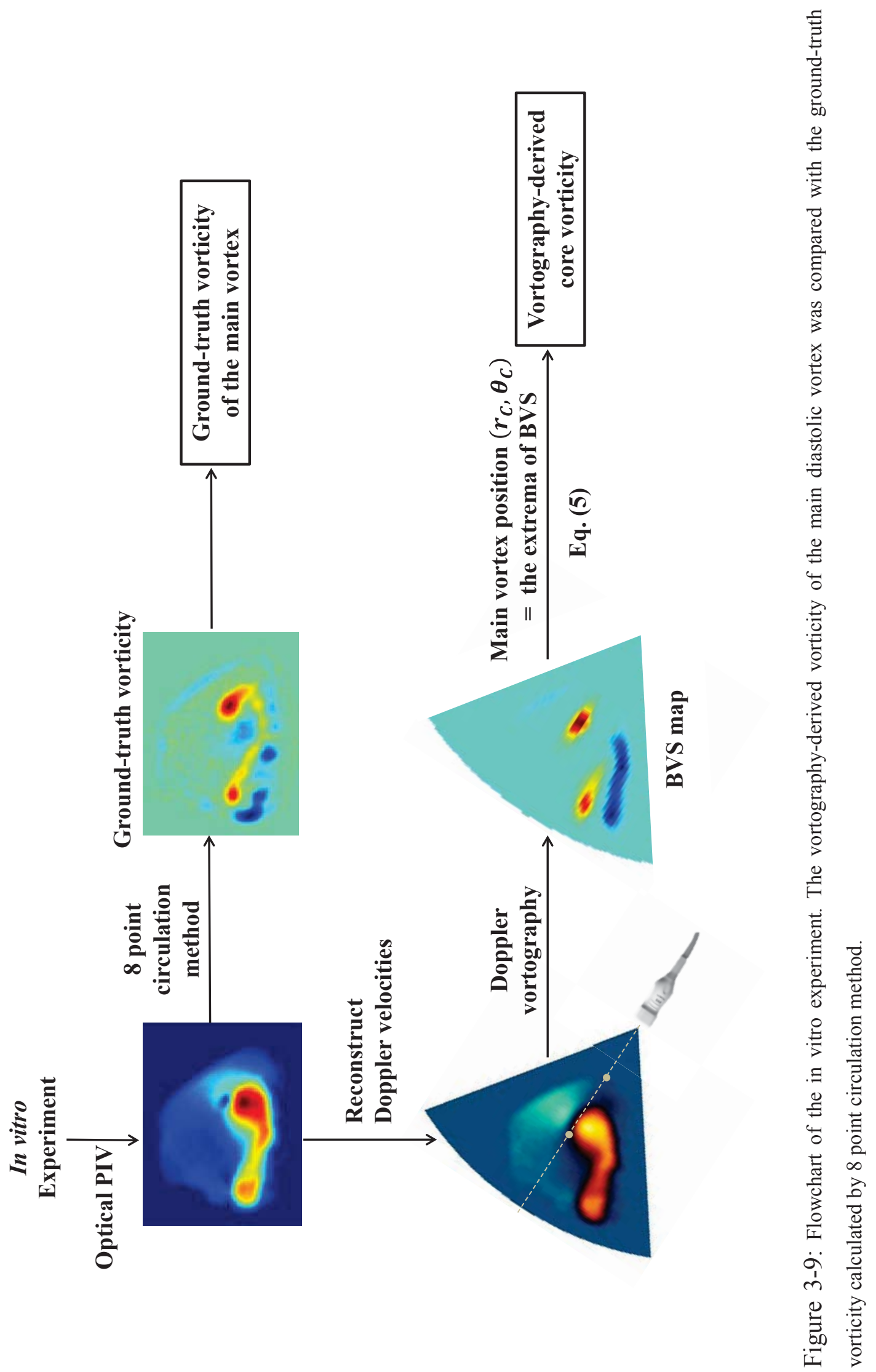
The vorticities estimated by Doppler vortography (vortography-derived core vorticity) were compared with those measured from the original PIV field provided by the reference method called the 8 point method [122] using a standard linear regression and a Bland-Altman plot. They were both measured at the same location, i.e. where the BVS amplitude was found maximal. This analysis was performed on the frames related to the diastolic filling only (total of 15 frames).

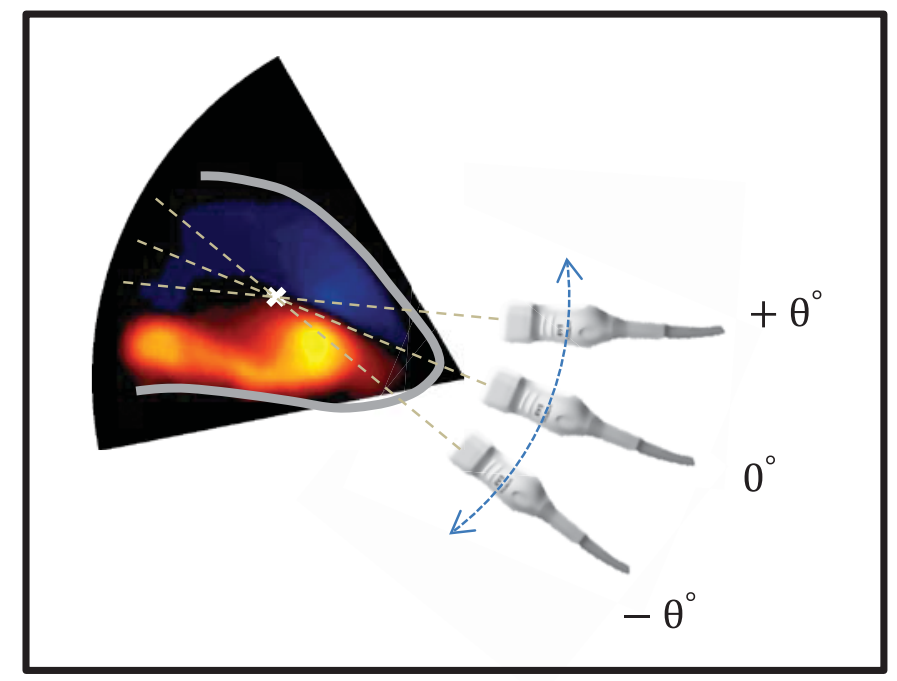

Figure 3-10: Effects of transducer position on the reconstructed Doppler velocity field in the in vitro experiment were analyzed. Several insonification angles $(0 \mathrm{o}, \pm 5 \mathrm{o}, \pm 10 \mathrm{o})$ were tested.

To check whether the transducer position (probe orientation) affects the results returned by Doppler vortography, several probe angles relative to the left ventricle centroid were tested $\left(0^{\circ}, \pm 5^{\circ}, \pm 10^{\circ}\right.$, see Figure $\left.3-10\right)$. The effects of the kernel size and the probe angle were analyzed independently using repeated measures analyses of variances by means of MedCalc software (version 12.6).

\subsubsection{In vivo experiments}

Some preliminary results issued from healthy subjects and patients with heart diseases were obtained to test whether this technique is readily applicable in clinical context. In this pilot study 18 patients belonging to two different groups were examined. Since hypertension is one of the main drivers of heart failure [131], hypertensive patients were considered in this study. Among these 18 
patients, 9 were considered as normal (no known cardiovascular disease or high blood pressure, normal ECG, normal echo surface study) and 9 were hypertensive with an expected mild to moderate diastolic dysfunction (normal LV size and mass, normal LV systolic function).

The exclusion criteria used to determine appropriateness for the clinical trial participation included age $<18$ years old, diabetes, permanent pacemaker, absence of sinus rhythm, prosthetic valve, any moderate to severe valve disease, medical history of cardiac ischemic event, chemotherapy or thoracic radiotherapy, severe pulmonary disease, moderate to severe renal failure, constrictive pericarditis, LV ejection fraction $<55 \%$.

A normal LV systolic function is defined as an ejection fraction $\geq 55 \%$, normal LV mass as $\leq 95 \mathrm{~g} / \mathrm{m}^{2}$ for women or $\leq 115 \mathrm{~g} / \mathrm{m}^{2}$ for men, and normal size of the $\mathrm{LV}$ as end diastole diameter $\leq 32 \mathrm{~mm} / \mathrm{m}^{2}$ for women and $\leq 31 \mathrm{~mm} / \mathrm{m}^{2}$ for men.

Subjects were included either by advertisement or as they present for routine tests in the echographic laboratory. Data was stored in our cardiac imaging database.

Doppler vortography was performed after post-processing of conventional colorDoppler data. This analysis was performed on the frames related to the early diastolic filling. The diastolic frames were specified by observing color Doppler data overlaid on B-mode images and also were checked using the electrocardiogram (ECG) of each subject (refer to Figure 1-2). Echocardiographic images of the left ventricle were acquired by means of a General Electric Vivid 7 (General Electric, Milwaukee, WI, USA) ultrasound machine using broadband 1.9 - 4.0 MHz transducers. Subjects were examined in the left lateral recumbent position using standard views (Figure 3-11). In order to apply our tools for vortex imaging, an apical 3-chamber view (Figure 1-15) from the apical position with color Doppler over the entire LV cavity was acquired. 


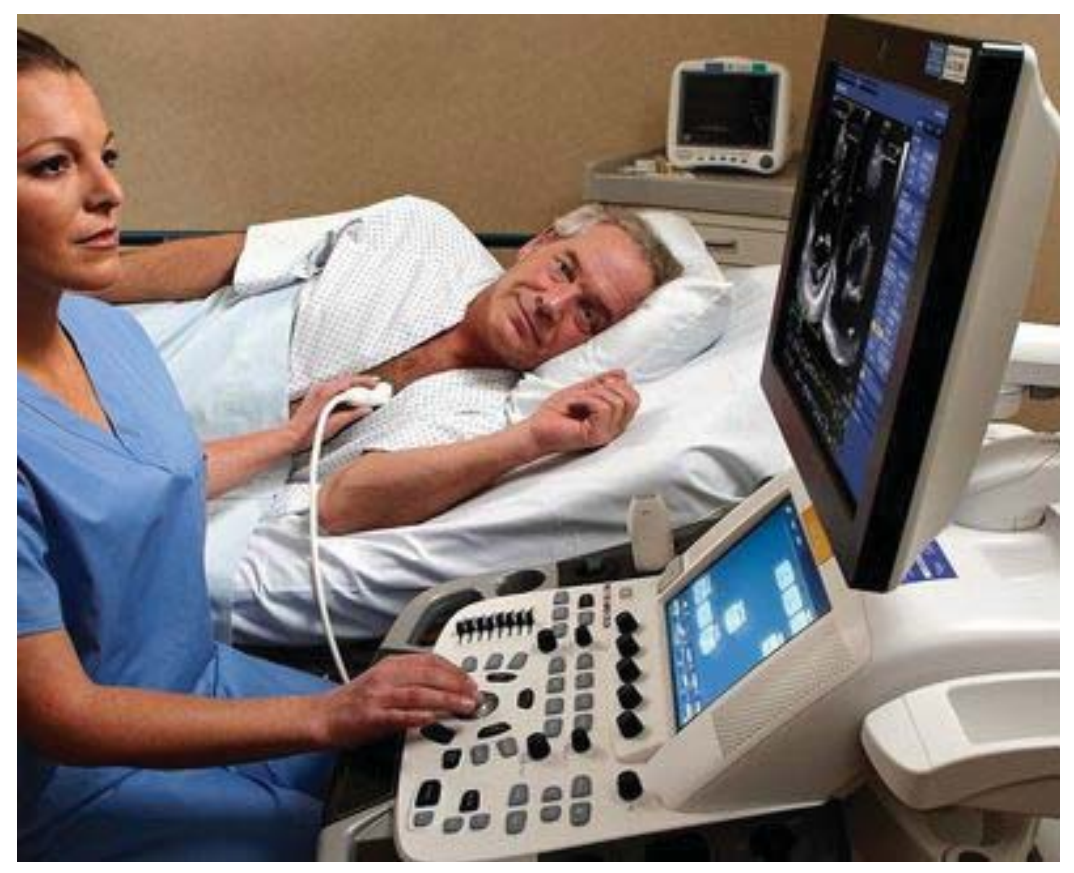

Figure 3-11: Patient in the left lateral recumbent position prepared for the echocardiographic examination (GE Healthcare, Vivid E9).

The imaging sector was adjusted to maximize the frame rate ( $>25$ frames $/ \mathrm{sec})$. At least three to ten complete cardiac cycles were acquired in the color-Doppler mode (refer to ECG trace in Figure 3-12). Echocardiographic raw data were extracted from the HDF5 (hierarchical data file) format using the clinical workstation EchoPAC (GE Healthcare). These data include all the information required for Doppler vortography: B-mode-derived ultrasound intensity, Doppler radial velocity, Doppler power, the position and size of the acquisition sector, the time samples, and the ECG signal.

To sum up, Doppler vortography interface works as follows:

1. Input the raw echocardiographic data (HDF5 file).

2. Choose an early diastolic frame (the scroll bar on top right of fig.3.12) according to the ECG trace and the B-mode image.

3. Perform Doppler vortography analysis and the results will be the vortex position and the corresponding core vorticity 


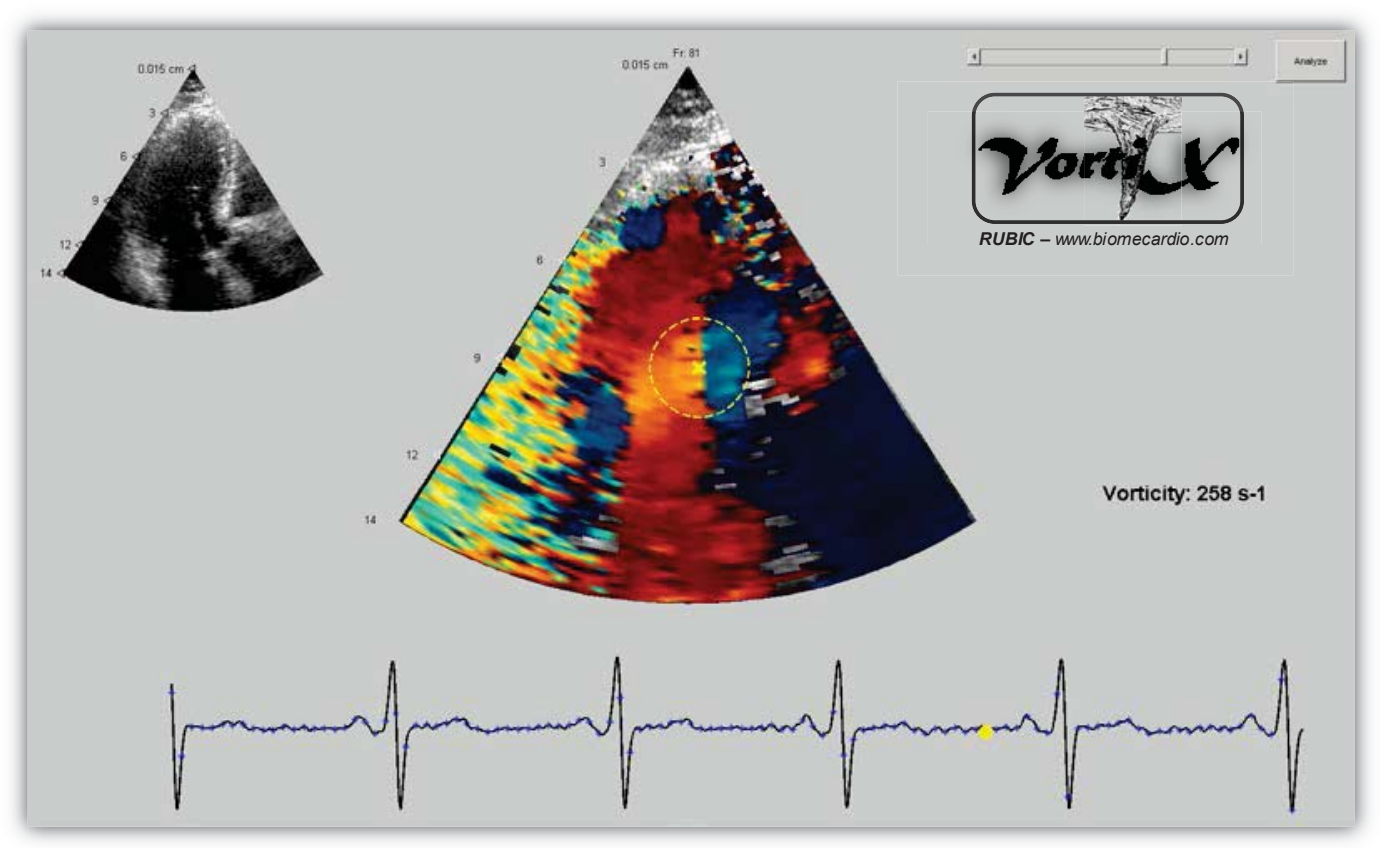

Figure 3-12: The graphical user interface for fast and easy detection and quantification of the intra-ventricular vortices by Doppler vortography. This interface is able to read and post-process the raw Doppler data issued from a GE ultrasound scanner. In this example the yellow circle represents the mitral vortex whose vorticity is $258 \mathrm{~s}^{-1}$.

In this part of the study, the following parameters have been evaluated using Doppler vortography: the presence or not of the apical vortex and the vorticity and size of the mitral and apical vortices (if any).

Sensitivity and specificity were calculated to test whether the presence of an apical vortex is a good indicator of diastolic dysfunction. The effectiveness of the diagnostic criteria was tested using a two-way contingency analysis by means of a Chi square test. The core vorticity of the mitral vortex was also measured for each heart cycle by Doppler vortography. The median values over 3 to 10 cycles were calculated and compared. Comparison of the core vorticities of the mitral vortices between the different clinical groups was performed by means of the ANOVA (analysis of variance). Other comparisons between two groups were made using Student T-test and a non-parametric Mann-Whitney U-test. 


\section{CHAPTER 4 RESULTS}

The reliability of Doppler vortography method was assessed in mock Doppler fields issued from numerical simulation and also PIV data in a left heart model. The potential clinical relevance of Doppler vortography was tested in two different groups. The corresponding results are presented in this chapter.

\subsection{In silico data}

\subsubsection{Doppler-derived vs. Ground-truth vorticity in silico}

The blood vortex signature was calculated from the post-processed Doppler data simulated by Field II software (Figure 4-1). The vortex core is located at the extremum of the BVS map. Kernel windows with different sizes $(3 \times 3,5 \times 5,7 \times 7$, $9 \times 9$, and $11 \times 11$ ) were tested (refer to Eq.3-2). The vortography-derived core vorticity (Eq.3-5) was compared with the ground-truth vorticity (Eq.3-11) for selected kernel sizes using a linear regression and Bland-Altman plot. The results are presented in Figure 4-2, Figure 4-3.

We observed a good concordance between the vorticities estimated by Doppler vortography (vortography-derived vorticity) and those derived from the control method (analytical solution, Eq.3-11). As it can be seen in Figure 4-2, high correlations were obtained in silico simulation for all kernel sizes $\left(r^{2} \sim 0.98\right)$. The relative errors in silico were also calculated using the Bland-Altman plots (Figure 4-3). As revealed by the Bland-Altman plot, small relative errors were observed in silico (i.e. $-6.8 \pm 10 \mathrm{~s}^{-1}$ for kernel size $7 \times 7$ ). 
8

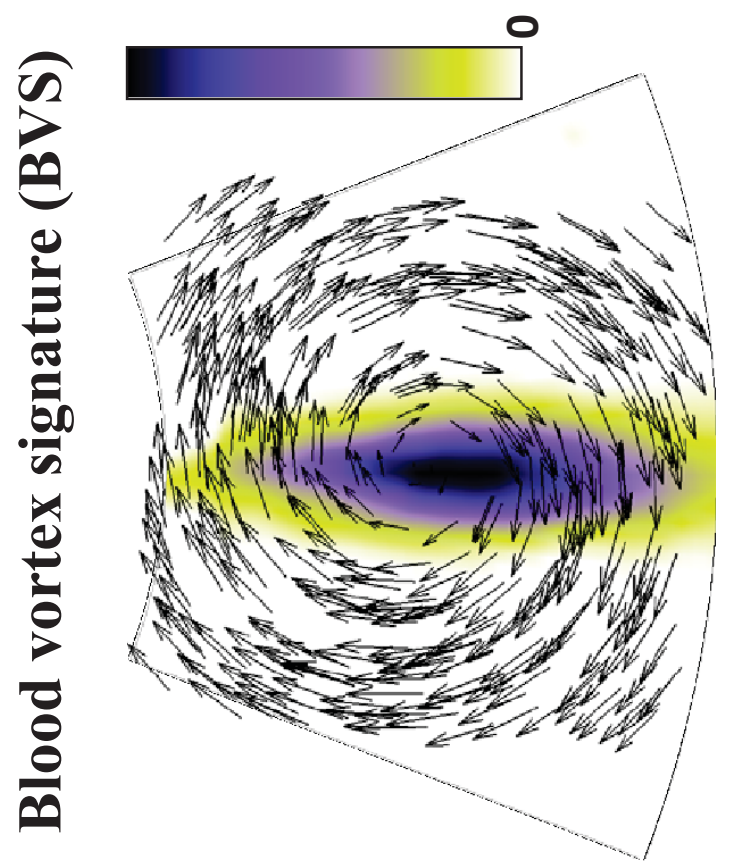

营

这

응

욤

宇

过

80

还

항

章

氜

过

$>$

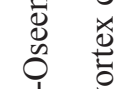

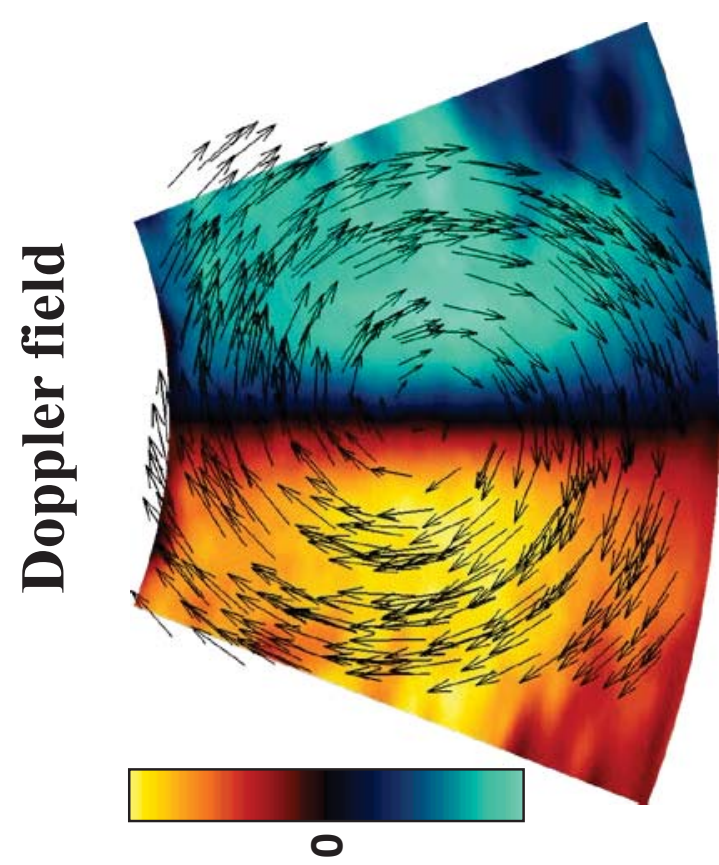

हี

昰

可苛

壱

灵

苛 స

है है

थे

के ठँ

के

ㅎํㅇ 䒕

نे

兽 范

总

$\Xi \pm$ ह

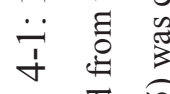

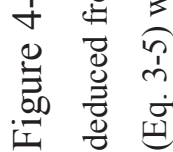


Kernel size $3 \times 3$

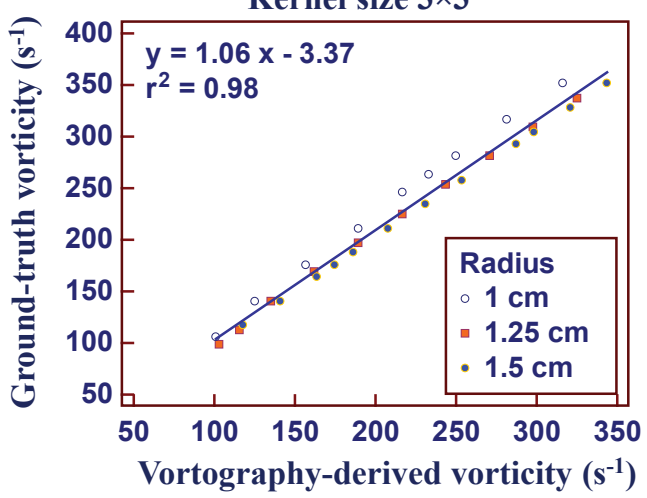

Kernel size $7 \times 7$

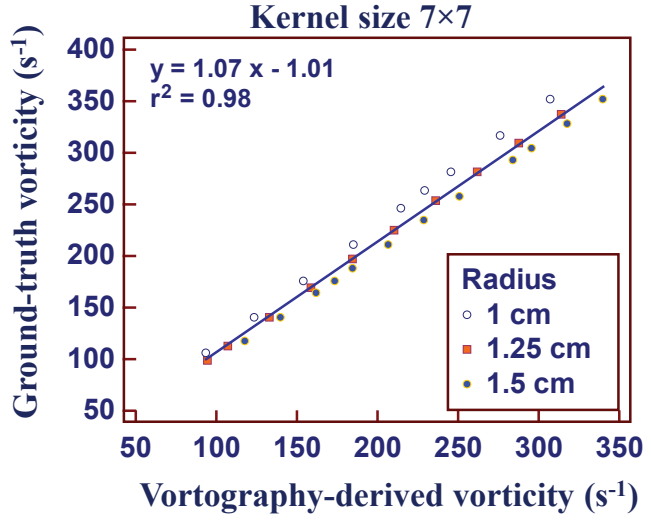

Kernel size $11 \times 11$

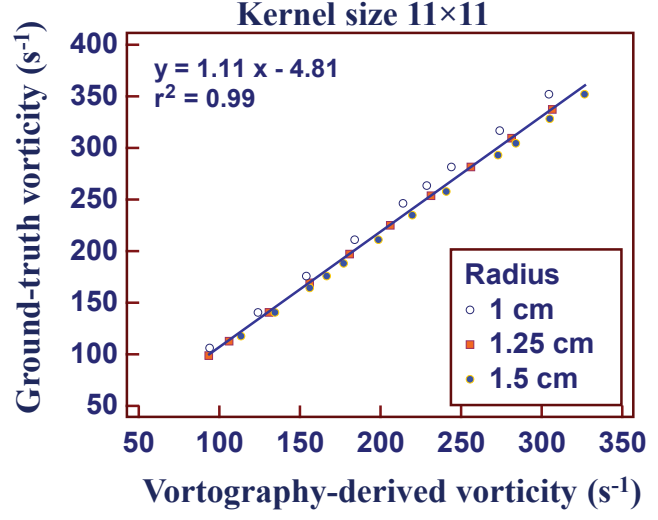

Kernel size $5 \times 5$

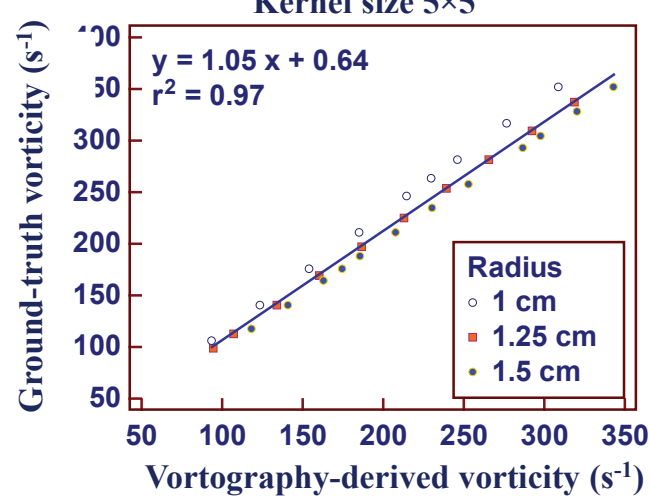

Kernel size $9 \times 9$

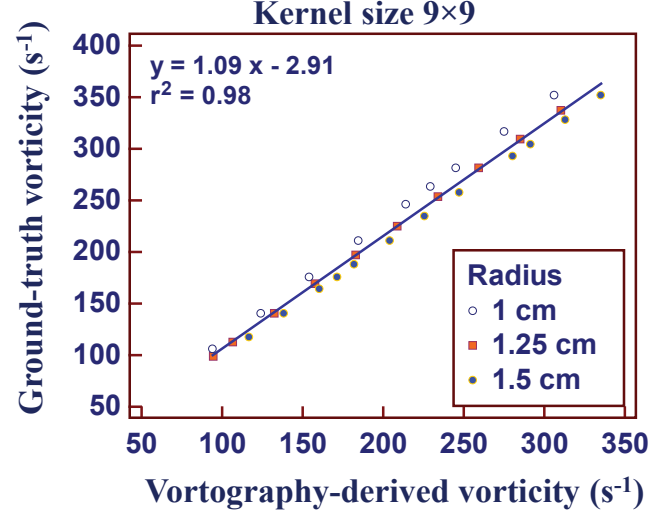

Figure 4-2: Concordance between the vortography-derived and ground-truth vorticities in silico for different kernel sizes using linear regression. 

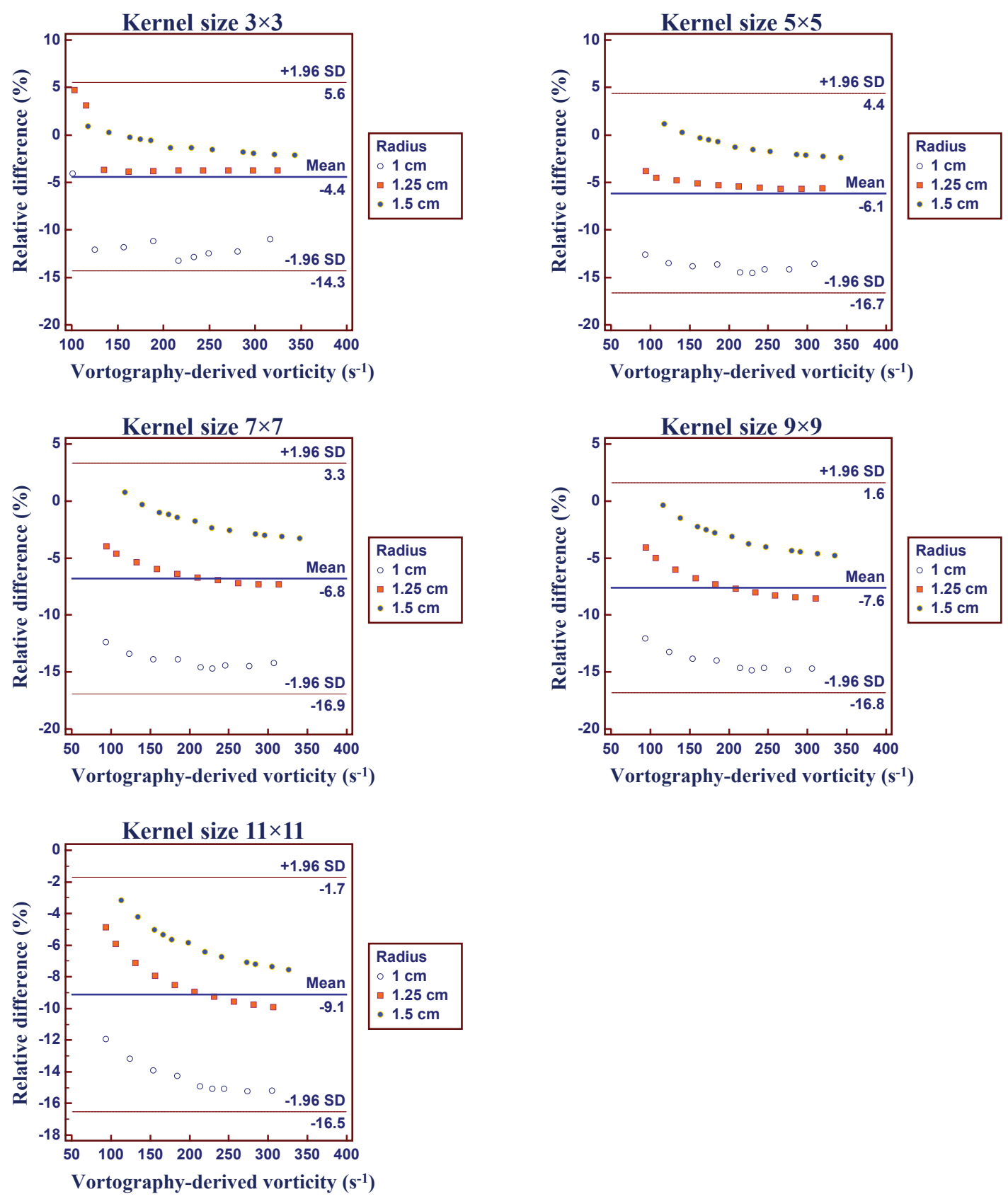

Figure 4-3: Relative errors in silico calculated for different kernel sizes using the Bland-Altman plot. 


\subsubsection{Effect of kernel size in silico}

The repeated measures ANOVA was used to test for differences among 5 groups of selected kernel sizes $(3 \times 3,5 \times 5,7 \times 7,9 \times 9$, and $11 \times 11)$. A significant effect of the kernel size $(\mathrm{P}<0.001)$ was reported in silico. There was a negative linear trend $(\mathrm{P}<0.0001)$ between the kernel size and the corresponding vorticity: i.e. the vorticity decreased with increasing Kernel size. Pairwise comparisons (the results are presented in table.4.1), however, showed that the mean vortcity differences between the $3 \times 3$ and $11 \times 11$ kernel sizes in silico were $9.25 \pm 0.82 \mathrm{~s}^{-1}$; this difference remained relatively small when compared with the actual vorticity values (mean $>200 \mathrm{~s}^{-1}$ ). Hence, the kernel size had negligible effect on the vortcity estimation.

Table 4.1: Pairwise comparison among 5 different kernel sizes in silico. The mean vorticity differences with standard errors are summarized.

\begin{tabular}{|c|c|c|}
\hline \multicolumn{2}{|c|}{ Kernel Sizes } & \multirow{2}{*}{$\frac{\text { Mean Difference }\left(\mathrm{s}^{-1}\right)}{2.848 \pm 0.482}$} \\
\hline $3 \times 3$ & $5 \times 5$ & \\
\hline & $7 \times 7$ & $4.369 \pm 0.543$ \\
\hline & $9 \times 9$ & $6.191 \pm 0.598$ \\
\hline & $11 \times 11$ & $9.251 \pm 0.820$ \\
\hline \multirow[t]{4}{*}{$5 \times 5$} & $3 \times 3$ & $-2.848 \pm 0.482$ \\
\hline & $7 \times 7$ & $1.521 \pm 0.244$ \\
\hline & $9 \times 9$ & $3.343 \pm 0.485$ \\
\hline & $11 \times 11$ & $6.403 \pm 0.898$ \\
\hline \multirow[t]{4}{*}{$7 \times 7$} & $3 \times 3$ & $-4.369 \pm 0.543$ \\
\hline & $5 \times 5$ & $-1.521 \pm 0.244$ \\
\hline & $9 \times 9$ & $1.822 \pm 0.272$ \\
\hline & $11 \times 11$ & $4.883 \pm 0.724$ \\
\hline \multirow[t]{4}{*}{$9 \times 9$} & $3 \times 3$ & $-6.191 \pm 0.598$ \\
\hline & $5 \times 5$ & $-3.343 \pm 0.485$ \\
\hline & $7 \times 7$ & $-1.822 \pm 0.272$ \\
\hline & $11 \times 11$ & $3.060 \pm 0.460$ \\
\hline \multirow[t]{4}{*}{$11 \times 11$} & $3 \times 3$ & $-9.251 \pm 0.820$ \\
\hline & $5 \times 5$ & $-6.403 \pm 0.898$ \\
\hline & $7 \times 7$ & $-4.883 \pm 0.724$ \\
\hline & $9 \times 9$ & $-3.060 \pm 0.460$ \\
\hline
\end{tabular}




\subsection{In vitro data}

\subsubsection{Vortography-derived vs. ground-truth vorticity}

As it has been previously discussed, the velocity fields in a left heart model for three different hemodynamic conditions were provided by PIV technique. Then the mock Doppler velocity fields were simulated by positioning the transducer in a $3 \mathrm{~cm}$ distance away from the apex of the left ventricle model. The noisy reconstructed Doppler velocity fields were post-processed by an unsupervised and easy-to-use algorithm called SMOOTHN [127, 128]. Then the BVS maps were calculated for different kernel sizes $(3 \times 3,5 \times 5,7 \times 7,9 \times 9$, and $11 \times 11)$. The vortex cores can be found at the local extremum of the BVS maps. Then the core vorticity at the position of the BVS extremums were calculated by Eq. (3-5). We mostly focused on the main diastolic vortex refer to as the good vortex (fig.2.5) in this document.

On the other hand, a control method is required to compare the simulated vorticities (Doppler-derived vorticity). As it is explained before, the control method called the 8 point method [122] utilizes the original two dimensional velocity fields for different hemodynamic conditions to calculate the core vorticity by means of Eq. (3-8) and (3-9) at the same position of the maximum BVS. These calculations were applied on the diastolic frames (total of 15 frames).

Figure.4.4 exemplifies the color Doppler field reconstructed from in vitro intraventricular PIV velocity field related to a diastolic frame. The corresponding PIVderived vorticity calculated by the control method and BVS measured by Doppler vortography are also shown in fig.4.4.B and $\mathrm{C}$ respectively. The local extremum of the BVS patch corresponding to the main diastolic vortex (see fig.4.4) was detected and the core vorticity was estimated. The Doppler vortography-derived vorticities were compared with PIV-derived vorticities using a standard linear regression. 
The effect of transducer position on the Doppler vortography method was tested by changing the probe angle relative to the left ventricle centroid. Five different angles and kernel sizes were considered in vitro experiment.

As it can be seen in fig.4.5, a high correlation was obtained for several probe angles in vitro $\left(r^{2} \sim 0.86\right)$. As revealed by Bland-Altman plot (fig.4.6), overestimations and higher relative errors were observed in vitro for different kernel sizes (i.e. $20.4 \pm 15 \%$ for kernel size $7 \times 7$ ).

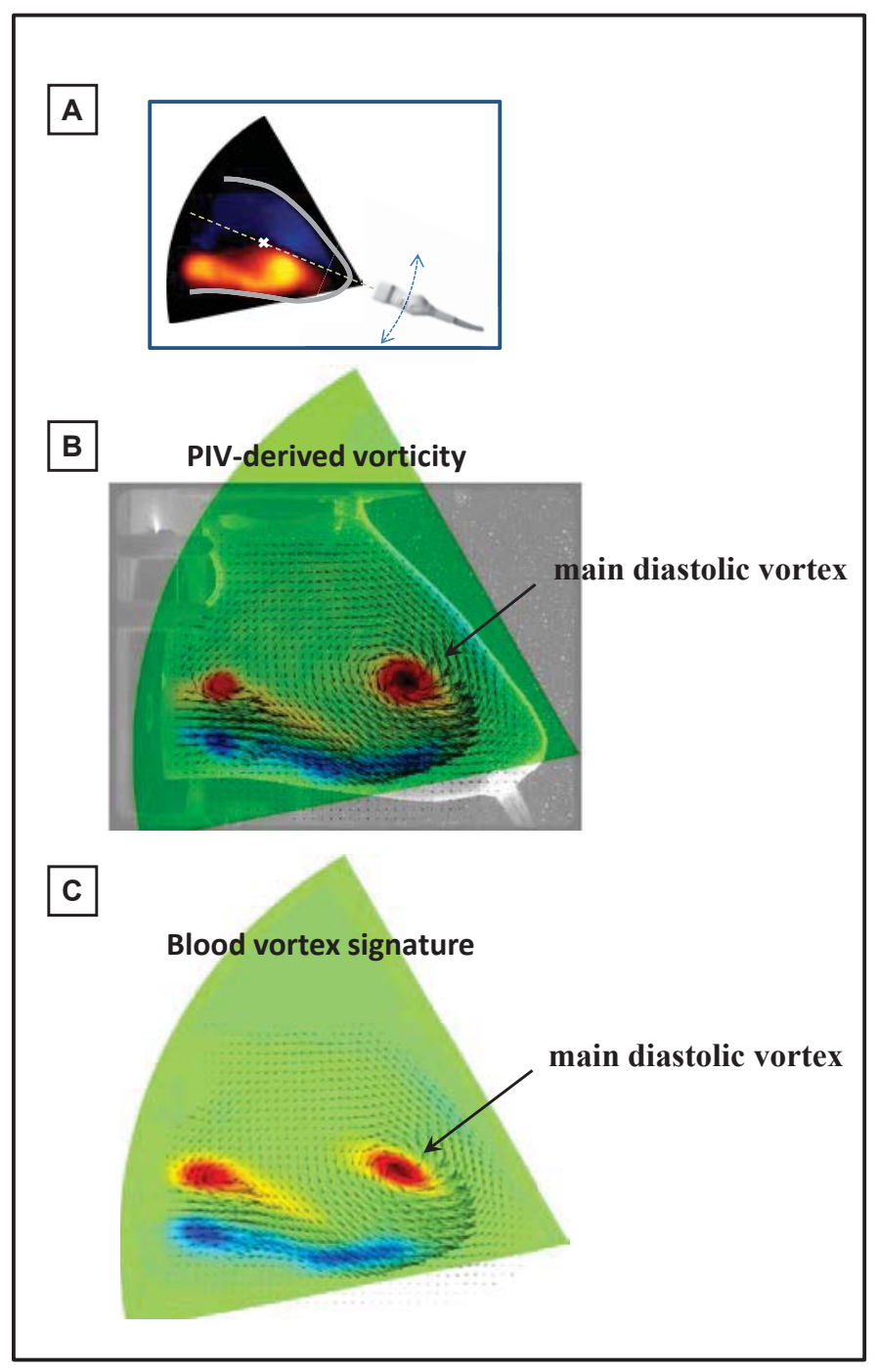

Figure 4-4: In vitro data. Color Doppler fields were created from in vitro intra-ventricular PIV (particle image velocimetry) velocity fields. The blood vortex signature was measured by Doppler vortography and the vorticity of the main diastolic vortex was compared with the PIV-derived vorticity. 

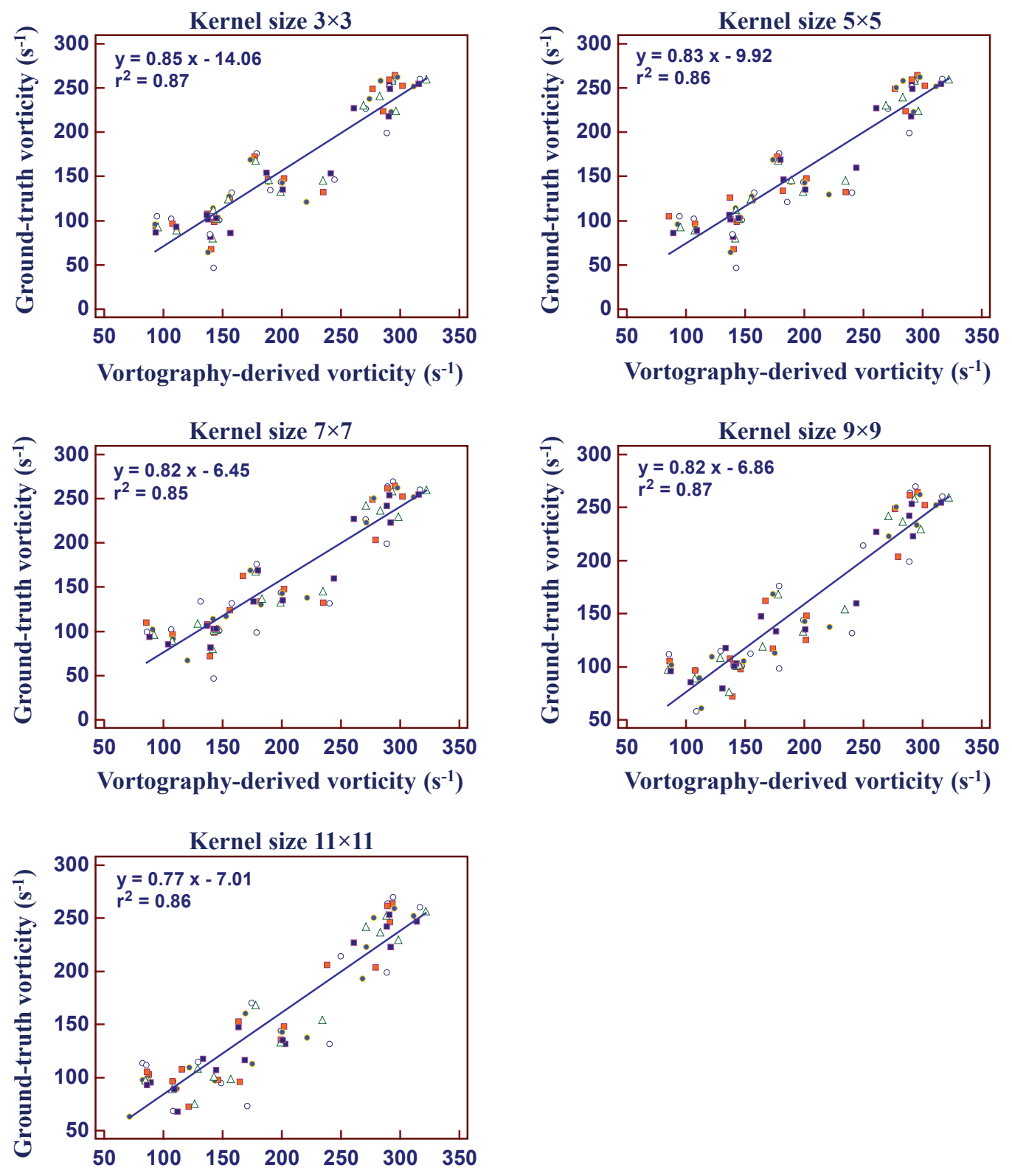

Vortography-derived vorticity $\left(\mathrm{s}^{-1}\right)$

Figure 4-5: In vitro results. Comparison between vortography-derived (Eq. 3-5) and ground-truth (Eq. 3-8 and 3.9) vorticities. Several angle insonifications (see fig. 3.10) were simulated for specified kernel window sizes. 

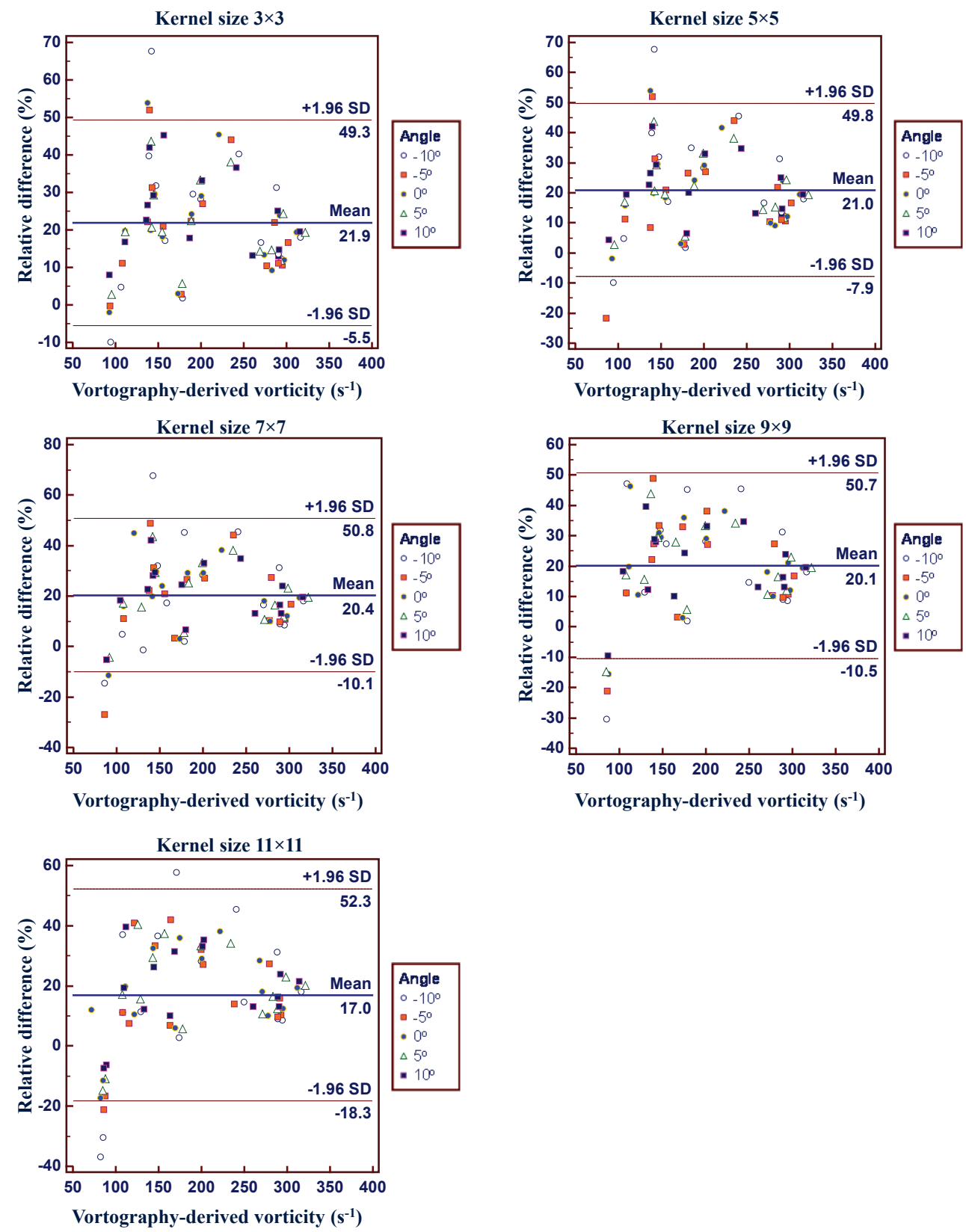

Figure 4-6: Relative errors in vitro calculated for different Kernel sizes using the Bland-Altman plot.

\subsubsection{Effect of kernel size and angle insonification}

The repeated measures ANOVA was used to test for differences among 5 groups of selected kernel sizes. A significant effect of the kernel size $(p<0.001)$ was observed in vitro. The measurements showed a negative linear trend $(p<0.0001)$ 
between the kernel size and the corresponding vorticities; i.e. the vorticity was decreased with increasing kernel size. In pairwise comparison, the mean vorticity differences between kernel configurations were compared to each other. The mean difference with standard error is given in table.4.2. It is shown that the mean vorticity differences between the $3 \times 3$ and $11 \times 11$ window sizes were $12.34 \pm 2.06$ $\left(\mathrm{s}^{-1}\right)$ in vitro. However, these differences are small compared to the actual vorticity value (mean $>200 \mathrm{~s}^{-1}$ ). The same procedure was performed to analyze the effect of beam orientation and the mean differences with standard errors are presented in table.4.3. No specific effect of the insonification angle $(\mathrm{p}>0.05)$ was reported for the in vitro data. It can be concluded that the kernel size and the insonification angle had negligible or no effect on the vorticity estimation.

Table 4.2: Pairwise comparisons using repeated measures ANOVA within 5 selected kernel sizes in vitro. The mean vorticity differences with standard errors are represented.

\begin{tabular}{|c|c|c|}
\hline \multicolumn{2}{|c|}{ Kernel Sizes } & \multirow{2}{*}{$\begin{array}{c}\text { Mean Difference }\left(\mathbf{s}^{-1}\right) \\
0.133 \pm 0.375 \\
\end{array}$} \\
\hline $3 \times 3$ & $5 \times 5$ & \\
\hline & $7 \times 7$ & $1.757 \pm 0.602$ \\
\hline & $9 \times 9$ & $4.623 \pm 0.941$ \\
\hline & $11 \times 11$ & $12.345 \pm 2.061$ \\
\hline \multirow[t]{4}{*}{$5 \times 5$} & $3 \times 3$ & $-0.133 \pm 0.375$ \\
\hline & $7 \times 7$ & $1.624 \pm 0.447$ \\
\hline & $9 \times 9$ & $4.490 \pm 0.924$ \\
\hline & $11 \times 11$ & $12.212 \pm 2.059$ \\
\hline \multirow[t]{4}{*}{$7 \times 7$} & $3 \times 3$ & $-1.757 \pm 0.602$ \\
\hline & $5 \times 5$ & $-1.624 \pm 0.447$ \\
\hline & $9 \times 9$ & $2.865 \pm 0.809$ \\
\hline & $11 \times 11$ & $10.587 \pm 2.012$ \\
\hline \multirow[t]{4}{*}{$9 \times 9$} & $3 \times 3$ & $-4.623 \pm 0.941$ \\
\hline & $5 \times 5$ & $-4.490 \pm 0.924$ \\
\hline & $7 \times 7$ & $-2.865 \pm 0.809$ \\
\hline & $11 \times 11$ & $7.722 \pm 1.889$ \\
\hline \multirow[t]{4}{*}{$11 \times 11$} & $3 \times 3$ & $-12.345 \pm 2.061$ \\
\hline & $5 \times 5$ & $-12.212 \pm 2.059$ \\
\hline & $7 \times 7$ & $-10.587 \pm 2.012$ \\
\hline & $9 \times 9$ & $-7.722 \pm 1.889$ \\
\hline
\end{tabular}


Table 4.3: The results of the pairwise comparisons using repeated measures ANOVA within 5 different insonification angles are represented. The mean vorticity differences with standard errors and P-value are given.

\begin{tabular}{|c|c|c|c|}
\hline \multicolumn{2}{|c|}{ Angles } & \multirow{2}{*}{$\frac{\text { Mean Difference }\left(\mathbf{s}^{-1}\right)}{2.819 \pm 1.204}$} & \multirow{2}{*}{$\frac{\text { P value }}{0.2146}$} \\
\hline$-10^{\circ}$ & $-5^{\circ}$ & & \\
\hline & $0^{\circ}$ & $2.596 \pm 1.031$ & 0.1358 \\
\hline & $+5^{\circ}$ & $-0.140 \pm 0.756$ & 1.0000 \\
\hline & $+10^{\circ}$ & $1.636 \pm 0.945$ & 0.8703 \\
\hline \multirow[t]{4}{*}{$-5^{\circ}$} & $-10^{\circ}$ & $-2.819 \pm 1.204$ & 0.2146 \\
\hline & $0^{\circ}$ & $-0.223 \pm 1.255$ & 1.0000 \\
\hline & $+5^{\circ}$ & $-2.959 \pm 1.212$ & 0.1659 \\
\hline & $+10^{\circ}$ & $-1.184 \pm 1.129$ & 1.0000 \\
\hline \multirow[t]{4}{*}{$0^{\circ}$} & $-10^{\circ}$ & $-2.596 \pm 1.031$ & 0.1358 \\
\hline & $-5^{\circ}$ & $0.223 \pm 1.255$ & 1.0000 \\
\hline & $+5^{\circ}$ & $-2.736 \pm 1.038$ & 0.0994 \\
\hline & $+10^{\circ}$ & $-0.960 \pm 1.258$ & 1.0000 \\
\hline \multirow[t]{4}{*}{$+5^{\circ}$} & $-10^{\circ}$ & $0.140 \pm 0.756$ & 1.0000 \\
\hline & $-5^{\circ}$ & $2.959 \pm 1.212$ & 0.1659 \\
\hline & $0^{\circ}$ & $2.736 \pm 1.038$ & 0.0994 \\
\hline & $+10^{\circ}$ & $1.775 \pm 0.909$ & 0.5383 \\
\hline \multirow[t]{4}{*}{$+10^{\circ}$} & $-10^{\circ}$ & $-1.636 \pm 0.945$ & 0.8703 \\
\hline & $-5^{\circ}$ & $1.184 \pm 1.129$ & 1.0000 \\
\hline & $0^{\circ}$ & $0.960 \pm 1.258$ & 1.0000 \\
\hline & $+5^{\circ}$ & $-1.775 \pm 0.909$ & 0.5383 \\
\hline
\end{tabular}

\subsection{In vivo study: Doppler vortography}

As it is previously discussed, Doppler vortography is a technique to specifically detect and quantify the intra-ventricular vortices that form during LV filling. An example of vortex detection by Doppler vortography is illustrated in Figure 4-7. The vortices are denoted by circles whose radii represent their strength. The direction of the vortices (clockwise and counter clockwise) is shown by curved arrows. Note that a small counter clockwise vortex may also appear adjacent to the posterior mitral valve leaflet. This is a normal vortex which was not studied in this research project. In this study we focused only on the mitral and apical vortices. 


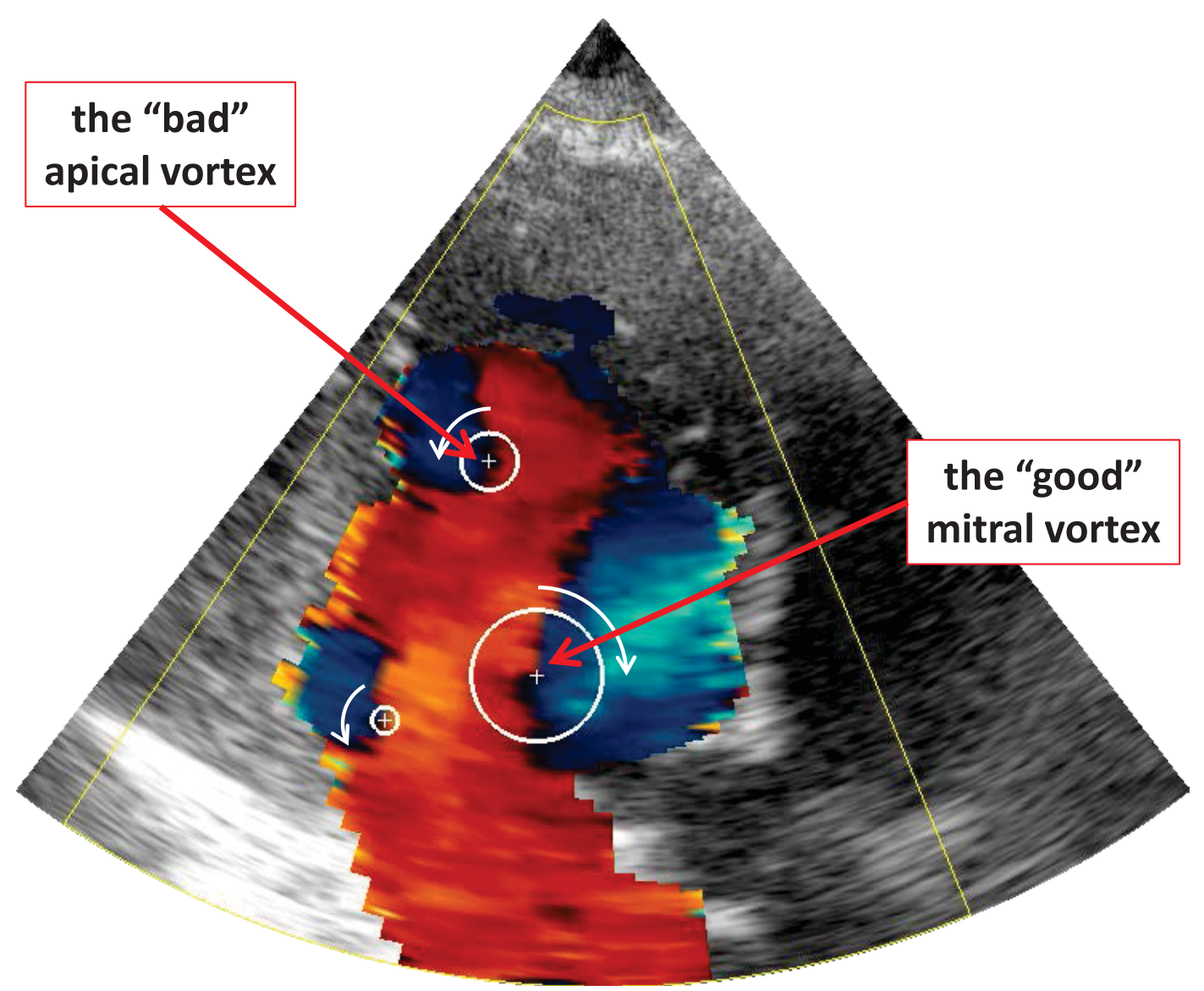

Figure 4-7: Vortex detection by Doppler vortography. Vortices are denoted by circles whose radii are related to the vorticity.

As a preliminary analysis, to test whether this technique is clinically compatible, Doppler vortography was performed on nine healthy subjects and nine hypertensive patients with normal LV mass and size using GE vivid 7. Following the steps mentioned in methodology sections (see also Figure 3-4 and Figure $3-12$ ), the position and size (strength) of the mitral and apical vortices can be evaluated.

Raw Doppler data and corresponding BVS maps of one normal subject (A) and two patients (B and C) are presented in Figure 4-8. As it can be seen, a clockwise vortex (represented by red) is present at the vicinity of anterior mitral valve in both healthy subjects and patients during early diastole. An abnormal counter rotating vortex (represented by blue) is also present near the apex in patients with cardiac diseases. 


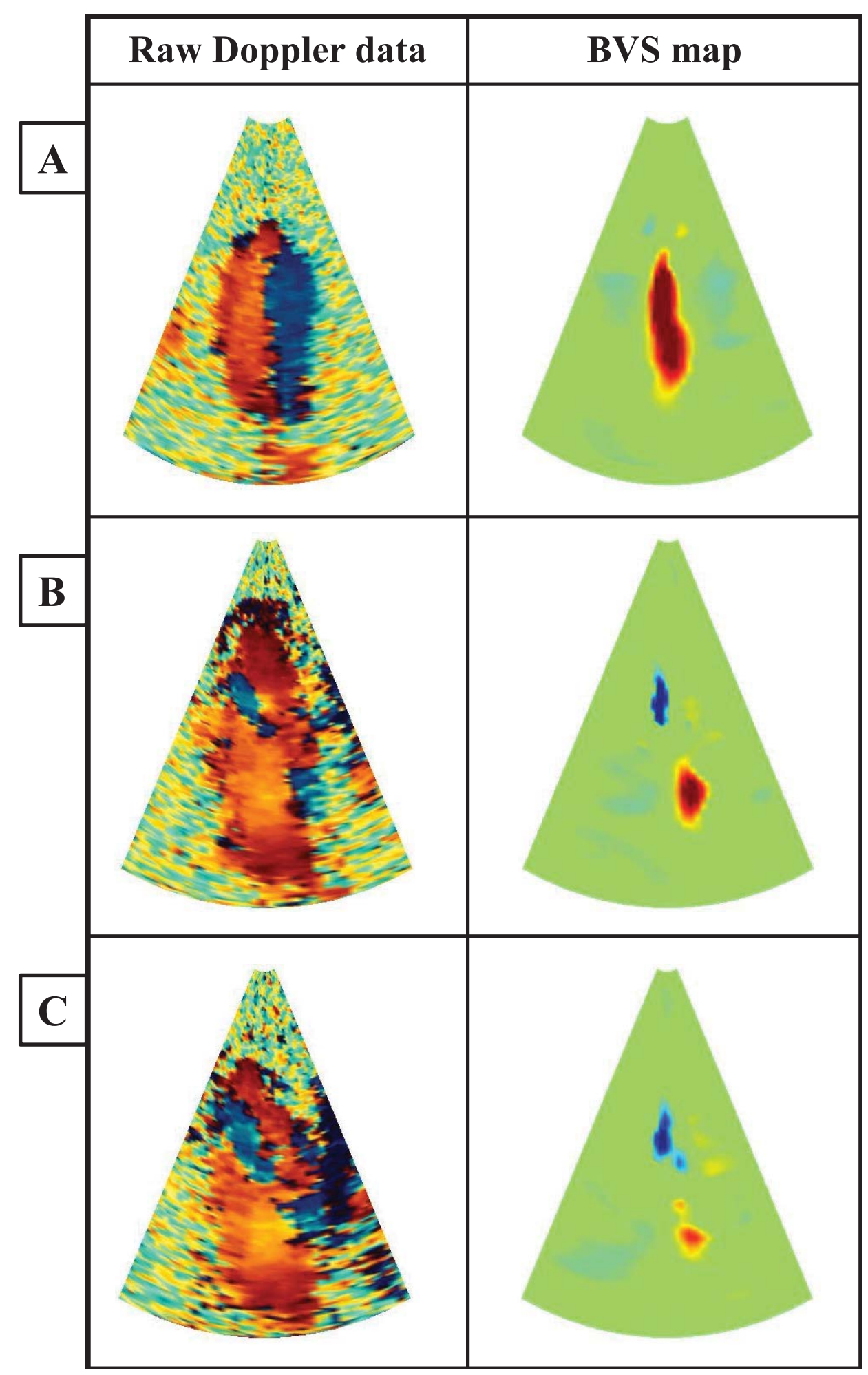

Figure 4-8: Raw Doppler data (left panel) and corresponding BVS maps (right panel) of three subjects (A. healthy, B and C. patients) during early filling. 
We detected an apical vortex in 7 hypertensive patients ( 7 true positives +2 false negatives) and one normal subject ( 8 true negatives +1 false positive) (see Figure 4-9). The accuracy of the apical vortex test is $83 \%$. The test sensitivity (the test ability to identify the presence of apical vortex in HTN patients) and specificity (the test ability to identify the absence of apical vortex in normal subjects) are $77 \%$ and $88 \%$ respectively. The effectiveness of the apical vortex test was evaluated using a two-way contingency analysis; a Chi square test returned a p-value of 0.004. In this small group we were able to detect an abnormal apical vortex in hypertensive patients.

\begin{tabular}{|c|c|c|c|}
\hline & \multicolumn{2}{|c|}{ Patients with HTN } \\
\hline & & Yes & No \\
\hline \multirow{2}{*}{ 胥 } & Yes & 7 & 1 \\
\hline & No & 2 & 8 \\
\hline
\end{tabular}

$$
\begin{aligned}
& \hline \mathbf{P}=\mathbf{0 . 0 0 4} \\
& \text { Sensitivity }=77 \% \\
& \text { Specificity }=88 \% \\
& \text { Accuracy }=83 \%
\end{aligned}
$$

Figure 4-9: Performance of the "apical vortex" test in normal and hypertensive (HTN) patients.

In the next step, the core vorticity of the mitral vortex (the good vortex) was also measured for each heart cycle by Doppler vortography and the median over 3 to 10 cycles were compared. We performed a two-tailed Student T-test and a nonparametric Mann-Whitney U-test to compare the medians of the normal and hypertensive groups. According to the presented results in Figure 4-10, there was a significant difference at the 5\% level between the two groups for both tests $(\mathrm{p}<$ 0.05). The mitral vortex (the good one) was weaker in hypertensive patients than normal subjects. These findings are very encouraging and of high interest for the routine echocardiographic assessment of diastolic function. These two promising observations tend to corroborate our hypotheses 1,2 and 3. 


\begin{tabular}{|c|c|}
\hline \multicolumn{2}{|c|}{ Mitral core vorticity $\left(\mathrm{S}^{-1}\right)$} \\
\hline $\begin{array}{c}\text { Normal } \\
\text { subjects }\end{array}$ & $\begin{array}{c}\text { Hypertensive } \\
\text { patients }\end{array}$ \\
\hline 161 & 107 \\
\hline 191 & 216 \\
\hline 96 & 140 \\
\hline 121 & 76 \\
\hline 159 & 108 \\
\hline 228 & 131 \\
\hline 286 & 80 \\
\hline 183 & 111 \\
\hline
\end{tabular}

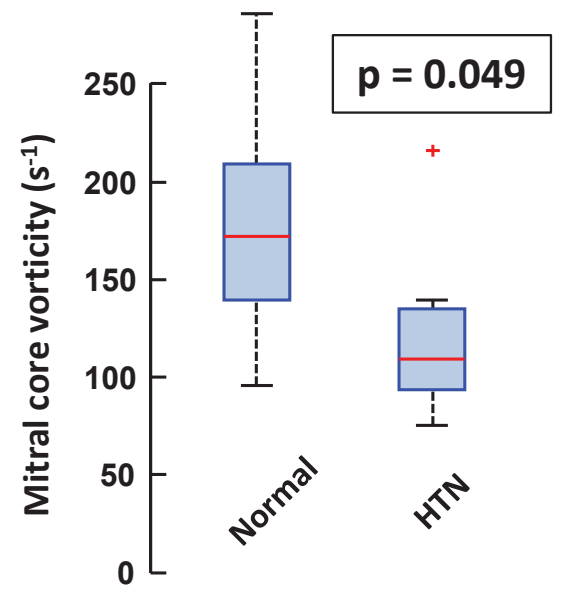

Figure 4-10: Left: Median of mitral core vorticities over 3 to 10 cycles for normal and hypertensive (HTN) groups. Right: Comparison of the mitral core vorticities between the normal and hypertensive groups. 


\section{CHAPTER 5 DISCUSSION}

\subsection{Presence of mitral and apical vortices}

It was the aim of this study to propose a new non-invasive index - Doppler vortography - based on vortical structures for assessment of the left ventricle function that can be easily applicable in a clinical context. Since this technique makes use of the conventional Doppler fields, Doppler vortography is a fully noninvasive, available and inexpensive modality to detect and quantify the intraventricular vortices that form during left ventricle filling. This method can be easily implemented for routine checks to recognize ventricular insufficiency and abnormal blood patterns at early stages of heart failure to decrease the morbidity of cardiac disease.

Doppler vortography modality for intra-ventricular vortex imaging was tested in 9 normal subjects and 9 patients, and the resulting blood vortex signature (BVS) revealed the presence of a clockwise rotating structure in contact with the anterior mitral valve leaflet (see Figure 4-8) both in healthy subjects and patients during diastolic filling. BVS mapping of three different subjects are also depicted in Figure 5-1. It confirms that only one diastolic vortex is present in normal subjects. This vortex is weaker in patients with severe cardiac dysfunction (see patient \#2 in Figure 5-1). This is probably due to the modification of the wall and mitral flow dynamics that significantly alters the intra-ventricular flow pattern. We observed that the diastolic mitral vortex can be easily detected at the end of early diastolic filling and before the atrial systole (at the onset of the E-wave deceleration). The smaller vortex adjacent to the posterior mitral leaflet was not always detectable depending on the cross-sectional view. Moreover, an additional abnormal counter rotating vortex was also present near the apex of the patients (see Figure 5-1 and Figure 5-2 and also Figure 4-8C). 


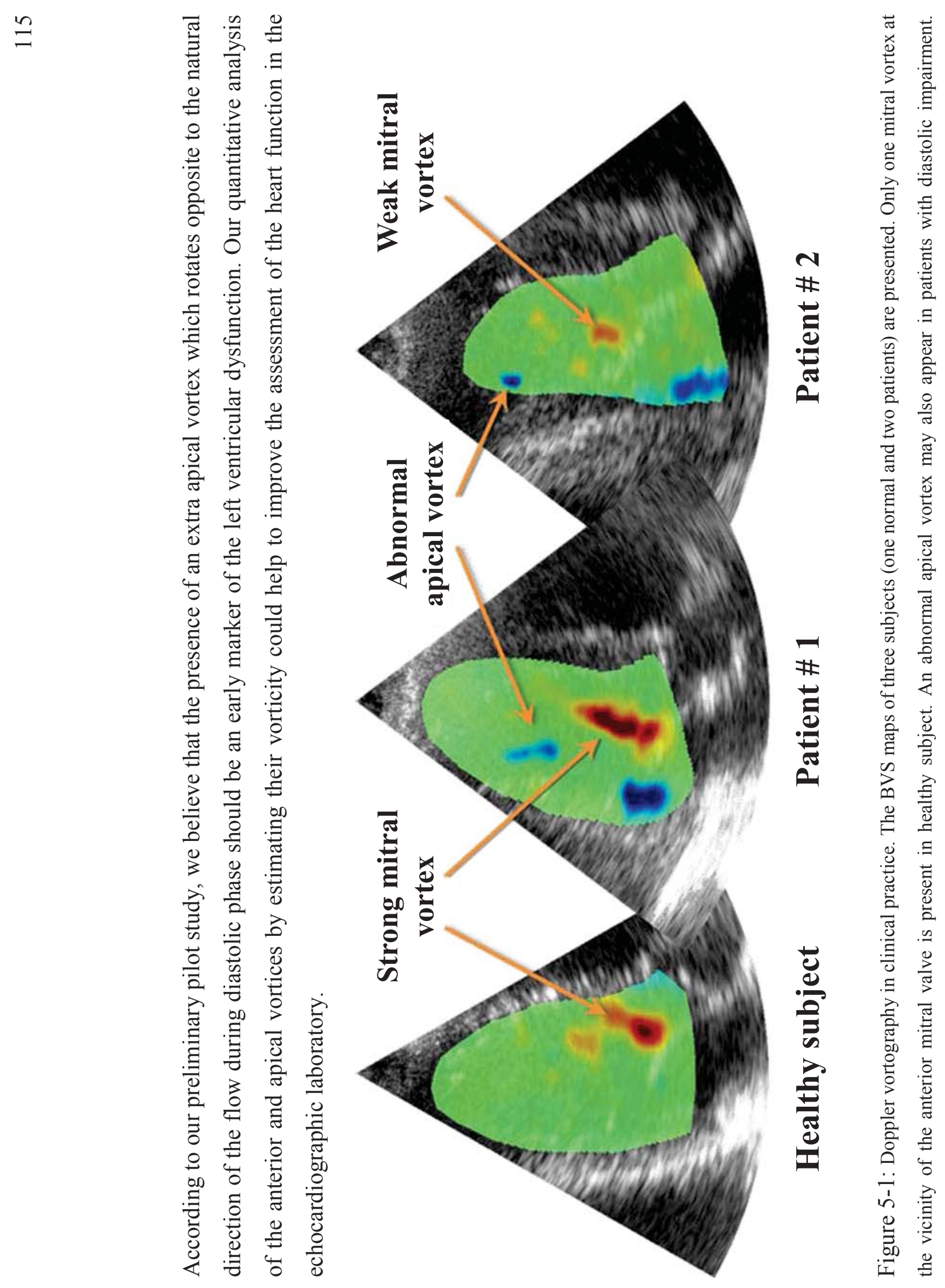


The recent research studies have also revealed an emerging clinical interest for characterization of intra-ventricular flow velocities and supported our findings in this research study $[1,2,45,46,62,63,85,86,94,132,133]$. Rodevand et al. described the flow velocity distribution within the left ventricle of normal subjects using color Doppler [46]. It has been shown that in early diastolic filling the blood flow velocities are directed toward the apex. At the end of the first diastolic pulse (at the peak of E-wave), the velocity gradient from the mitral valve to the apex tends to decrease leading to a partial closure of the anterior mitral valve leaflet. Right at the peak of E-wave, two vortices appear at the tip of the anterior mitral leaflet (major vortex) and behind the posterior one. Although the filling velocities are very low during diastasis, the major vortex (only) persists in the left ventricle. At the end of atrial contraction wave (A-wave), the major vortex pushes the mitral valve backward for closure. Moreover, in some patients, relatively apically located vortices were seen. The anterior vortex formation within the left ventricle of healthy subjects during mitral inflow has also been investigated by Kim et al. [94] using MRI. During E-wave deceleration when the anterior leaflet moves toward the partial closure, the valve is brought into a relevant place for formation of the shear layer between the transmitral flow and the leaflet. The shear layer creates a vortex at the leaflet tip which rolls-up into the low pressure region behind the anterior leaflet due to the inward motion of the valve. Then the anterior vortex expands and directs the flow towards the apex and the outflow tract with the continuous ventricular diastolic filling. A weak transient recirculation may also be seen beneath the posterior mitral valve leaflet. Kim et al. reported that the anterior vortex is formed after the beginning of the mid-diastolic partial mitral valve closure and arises again at the instance of the final mitral valve closure [94]. However, Sengupta et al. [107] showed the continued presence of anterior vortex on the duration of the ventricular filling phase by performing echo contrast particle image velocimetry (echo-PIV) in 10 beating pig hearts. Similar vortex flow pattern was detected immediately after the onset of early diastole in normal subjects using echo-PIV performed by Hong et al. [45]. This circulating flow pattern persists until the end of the diastolic filling and dissipates with the aortic valve opening 
during ventricular ejection. Furthermore, the position and the strength (vorticity) of this vortex are dynamically dependent on the phase of the heart cycle. In the case of ventricular dysfunction, the mitral vortex seems to be incoherent with lower vorticity than normal case. The swirling flow during ventricular diastole was also tested by Mouret et al. [134] using laser PIV in an atrioventricular model. Their results reveal the presence of two vortices at the tip of the mitral leaflets before the deceleration of the E-wave. The vortex formation time is slightly earlier than the in vivo findings by Kim et al. [94]. This might be due to the higher shear layer and vorticity at the leaflet surface in vitro. The well-developed circular mitral flow pattern was also observed at the onset of the filling phase in an in vitro PIV examination by Garcia et al. [63]. Preliminary clinical Doppler results using Garcia's method [63] were consistent with the previous studies using MRI techniques $[1,60,93]$ and confirmed the swirling nature of the intra-ventricular flow. At the beginning of the E-wave deceleration stage, the large diastolic vortex starts to appear at the edge of the anterior leaflet while its strength and size keeps rising during the whole diastole. In patients with ventricular abnormalities such as hypertrophic and dilated hearts, the major vortex appears to be disorganized (grossly disturbed especially in dilated ventricle) in compare to normal subjects along with the presence of the extra recirculating patterns near the apex of left ventricle. It is worth mentioning that the mitral vortex is stretched during systolic contraction and is ejected and disappears almost completely before the onset of diastolic filling. Therefore, the remaining vorticity from the previous cycle weakly affects the mitral inflow vortex and the diastolic flow can be considered vortexfree before the beginning of ventricular filling $[72,135]$.

Our in vivo results (see Figure 4-8) using Doppler vortography unveiled that in most of patients, a counter-rotating vortex with respect to the natural flow was visible near the apex. In others (such as in Figure 5-2), a large vortex was present adjacent to the posterior mitral valve leaflet (also refer to Figure 4-7). In patients with abnormal heart function like acute myocardial infarction, there is a wall insufficiency that disrupts the flow causing the geometry of the vortex to change 
and also the appearance of undesirable vortices. In the presence of an abnormal vortex near the apex, the left ventricle may enter into a vicious circle. Since this additional apical vortex rotates in a direction opposite to the natural circulation path, it induces excessive fluid energy dissipation and thus increases the cardiac workload and negatively affects the myocardial efficiency. In addition, this apical vortex may disturb the intra-ventricular flow pattern which in turn decreases the vorticity of the mitral vortex (see Figure 4-8). It could be expected from our in vivo results (refer to Figure 4-10) that the vorticity of the diastolic mitral vortex is, to some extent, negatively related to the degree of the filling impairment; weaker mitral vortex shows the more severe level of ventricular dysfunction.

Recent in vitro and in vivo observations also confirm that the mitral vortex might minimize the fluid energy dissipation and optimize the LV myocardial efficiency $[1,2,133,136]$. Charonko et al. [133] studied the blood flow in the left ventricle during filling in normal subjects and patients with abnormal diastolic function using a 2D PCMRI and measured the strength and location of the intra-ventricular vortices. The corresponding results revealed that the formation of the mitral vortex assists ventricular filling by reducing convective losses and optimizes the LV function as a suction pump. Any abnormality or malfunction in this mechanism contributes to diastolic dysfunction and consequently severe heart failure. The analysis of the flow patterns in a left heart numerical model performed by Pedrizzeti and Domenichini [2] verifies that the energy dissipation within the left ventricle is significantly affected by the vortex flow arrangement. The intraventricular rotating structure is considered to store some kinetic energy during diastole and facilitate systolic ejection by diminishing the energy that the myocardial muscle must produce to eject the flow through the outflow tract. The presence of an unnatural vortical flow pattern may result in an increase in energy dissipation and cardiac muscle workload and as a consequence a reduction in the pumping efficiency of the heart by over 10 percent [2]. 


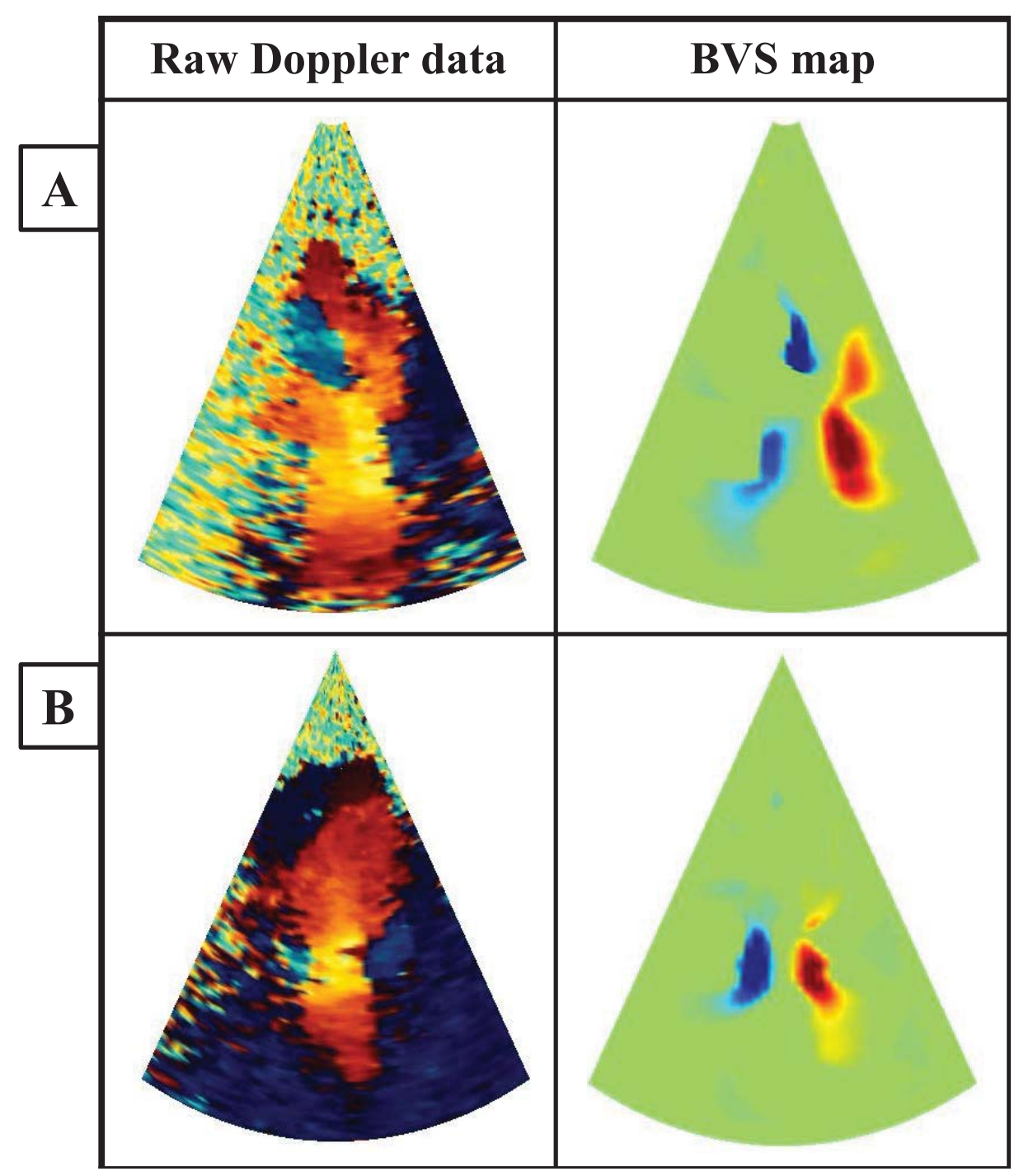

Figure 5-2: Raw Doppler data (left panel) and corresponding BVS patches (right panel) of two patients during early filling. An extra counter-rotating apical vortex was visible in most of the patients during diastole (A). In some patients a large vortex was present adjacent to the posterior mitral valve leaflet (A and B).

Besides in vivo results, our finding from numerical results and in vitro simulations clearly demonstrate that Doppler vortography can provide reliable measures of left ventricle vortices during diastolic filling. The presented in vivo study was a very preliminary analysis on 18 patients to test whether Doppler vortography is clinically compliant. At this step of the project, our assertions (weaker mitral vortex and presence of the apical vortex in patients with cardiac disease) remain hypothetical. It requires further confirmations by means of a prospective study in a 
sufficiently large group of subjects. This will assist us to better appreciate the potential clinical interest of Doppler vortography in detection and quantification of diastolic anterior and apical intra-ventricular vortices for the assessment of left ventricular function.

\subsection{Why using two-dimensional assumption?}

In this study we deliberately selected the apical long-axis 3-chamber view to visualize and quantify the large vortical structures occurring in this plane for normal subjects and patients. This plane passes through the LV apex and the centers of the mitral and aortic valves. According to the literature, the intra-cardiac vortices have been previously detected in two dimensional visualization using echocardiography and conventional 2D cine PCMRI techniques. Although intracardiac flow is known to be $3 \mathrm{D}$, flow measurements with phased contrast MRI revealed that it can be simplified to a two dimensional planar flow in an apical long-axis view while obtaining the important parameters that reflect the hemodynamics of intra-ventricular flow [47, 137]. Thompson and McVeigh [137] proposed a new method with PCMRI for fast non-invasive estimation of the intracardiac pressure differences using only the in-plane velocity components. They showed that accurate acquisition of pressure differences within a slice of interest is guaranteed because the momentum fluxes normal to the plane of interest is negligible. By neglecting the contributions from the through-plane velocities in $2 \mathrm{D}$ PCMRI imaging, fast acquisition and processing time with smaller data set along with a good spatial and temporal resolution is expectable. Phase-contrast MR studies have shown that the out-of-plane velocity components are small in the plane corresponding to the echo 3-chamber view [93, 138, 139]. Erikson et al. [138] developed a semi-automatic analysis for quantification of 4D ventricular flow pattern. Although, this 4D method can accurately measure the stroke volume and end diastolic volume, it is not a valid substitute technique for previous $2 \mathrm{D}$ cine through-plane phased-contrast MRI or ultrasound methods for the assessment of these parameters [138]. This method has been applied in patients with 
compensated HF [139]. The corresponding results indicate that the accuracy of 4D PCMRI is constrained in the presence of eddies and vortex patterns in the flow. In addition, vortex patterns can be missed in four- and two-chamber views in some subjects [92]. A detailed comparison of 3D and 2D cine PCMRI performed in vivo reveals that the 3D cine PCMRI provides slightly lower velocities [140]. This systematic underestimation is most likely due to lower spatial resolution in $3 \mathrm{D}$ cine PCMRI. In addition, Garcia et al. [63] developed a novel echocardiographic technique for imaging the blood velocities in the left ventricle based on a two dimensional assumption. Although intra-ventricular flows occur in three dimensions in space, they believe that the important flows are approximately 2D in the plane of interest (3-chamber view).

The consensus from a majority of these independent studies is that the utilization of $2 \mathrm{D}$ imaging in particular apical long axis 3-chamber view is quite acceptable for the analysis of the main diastolic vortices. In addition, clinical and functional parameters of the flow dynamics within the LV can be evaluated using this typical two dimensional flow simplification. Although LV dynamics is undoubtedly three dimensional, we neglected the through-plane displacements and thus assumed that the main flow arrangements remain measurable in the 3-chamber view without significant loss of information.

\subsection{Technical limitations of the study}

Because Doppler vortography is based on two-dimensional color Doppler, it is affected by common artifacts arising in Doppler ultrasound imaging such as aliasing, low SNR, low angular and temporal resolutions and clutter signals. As previously explained, the low frequency signals produced by low velocity structures are much stronger than the signals from the blood and must be removed and supressed by means of the clutter filtering. This high pass filter removes the low frequencies below a certain cut off frequency point. However, inappropriate setting in clutter filtering may remove the signals from low velocity blood flow. In this study the default clutter filter, provided by the clinical GE ultrasound scanner 
was used during the acquisitions in patients. Since we were interested in the frames with relatively high inflow velocities (E-wave), the cut off frequency point could not affect the results returned by Doppler vortography. Also, signal-to-noise ratio was high for most of cases during early filling. An unsupervised postprocessing technique developed by Muth et al. [127] was performed to dealiased and denoised the low SNR Doppler images with high accuracy. In addition, according to sampling theorem a signal can be reconstructed from its samples, if the sampling frequency is greater than twice the maximum frequency of the original signal. Hence, under-sampling could also hinder the vortex detection and quantification by Doppler vortography. The Doppler sector for each patient contained at least 50 scanlines and was set to cover the entire inner left ventricular cavity to ensure high-quality measures. It is known that the number of scanlines in a Doppler sector limits the frame rate and if a greater sector width is required in a few cases, it has to be compensated by lower frame rate. Therefore, high-quality images were sometimes at the expense of frame rate. However, low frame rates do not affect our technique by itself. It is worth mentioning that after some practice, the vortex region can be detected by an experienced eye which will definitely help to better adjust the Doppler sector.

\subsection{Future perspectives}

\subsubsection{Future validations}

\subsubsection{3D in vitro experiments}

A 3D PIV setup will be used to validate whether Doppler vortography can quantify the vortices accurately and provide precise measures of core vorticities. Optical PIV and Doppler measurements will be performed in a ventricular phantom (ViVitro Labs Inc, Canada) under variable hemodynamic conditions. In this in vitro model, the LV wall will be compliant and transparent. A liquid made up of glycerol and saline water will be used to reproduce blood viscosity and density. Several ventricular geometries (various shapes and thicknesses) will be 
designed to simulate normal and diseased ventricles. The intra-ventricular velocity field will be measured by means of the V3V system (TSI Inc., MN, USA) which is the only volumetric three components velocimetry system. The truly volumetric LV flow will be highlighted with double-pulse laser synchronized to a high speed camera for capturing two consecutive PIV frames. The image series will be processed using the TSI software package and 20 to 30 consecutive heart cycles will be considered to obtain ensemble average velocity fields. Color Doppler acquisitions will be performed immediately after PIV measurements. Echographic acquisitions of LV will be performed in the long-axis 3-chamber view from the apex using a portable Vivid i ultrasound scanner. The core vorticities measured by Doppler vortography will be compared with the ground-truth vorticities provided by optical PIV technique using the 8-points method (refer to section 3.1.2). The vortices provided by 3D PIV and Doppler vortography will also be compared in terms of the center position. Several physiological and pathophysiological conditions (stroke volume, peak LV pressure, heart rate, and ejection fraction) will be investigated. The ground-truth and vortography-derived vortex properties (position, vorticity) will be compared using standard statistical techniques. We anticipate that Doppler vortography will remain precise in $3 \mathrm{D}$ measurements.

\subsubsection{Phased contrast MRI in volunteers and patients: Doppler vortography vs. MRI}

MRI is the only modality that provides comprehensive and accurate threedimensional information on structural and flow dynamics within cardiac chambers. The potential error of the Doppler vortography technique related to the $2 \mathrm{D}$ flow assumption will be tested in a set of phase-contrast MRI studies in volunteers and patients with cardiac dysfunction. Three-dimensional velocity profile within the LV can be obtained by PCMRI. The vorticity obtained by Doppler vortography can be compared with vorticity obtained by the true velocity (3D) components provided by magnetic resonance imaging. The $2 \mathrm{D}$ assumption and neglecting the contributions from the through plane velocity components in Doppler vortography technique will also be tested. 


\subsubsection{Clinical interest}

\subsubsection{Validations in animal models}

To verify whether the vortex properties (core vorticity and presence of atypical votices) given by Doppler vortex flow imaging (Doppler vortography) can better predict the diastolic function, we need to validate and compare them against the gold-standard catheter-derived indices of left ventricular diastolic function in animal models. The pig model is one of the most widely used animal models in cardiac researches. In this study three groups of mini-pig (group 0: control, group 1: chronic LV pressure overload and LV hypertrophy, and group 2: acute LV pressure overload) will be created. In this invasive technique, LV pressure-volume (PV) loops (refer to section 1.2.1.1.1) will be acquired using a pressure conductance catheter inserted invasively from the right carotid artery. The most common gold-standard indices of LV diastolic function such as end diastolic pressure volume relationship (EDPVR) and chamber stiffness will be determined by Matlab home-made programs. Echocardiographic measurements will be performed using a portable Vivid i system and a 1.5-4 MHz phased array probe. To obtain optimal ultrasound signals and to avoid the strong attenuation due to pig skin, the abdominal cavity will be accessed by laparotomy and the probe will be placed against the diaphragm. The proposed vortex imaging parameters (core vorticity and position) will be assessed in all groups along with systolic and diastolic functions (i.e. $\mathrm{E} / \mathrm{A}, \mathrm{V}_{\mathrm{p}}, \mathrm{E} / \mathrm{V}_{\mathrm{p}}, \mathrm{E} / \mathrm{e}^{\prime} \ldots$...). The vorticities estimated by Doppler vortography will be compared with the catheter-based indices of diastolic function. To check whether vortography-derive mitral and apical vorticities can be used as non-invasive indices of diastolic function, these parameters will be compared with the gold-standard catheter-based indices of diastolic state such as EDPVR and chamber stiffness. We expect that the mitral vortices should decrease in groups 1 and 2 . In addition, we anticipate that an apical vortex will be also present in hypertrophic pigs. 


\subsubsection{Prospective clinical study in hypertensive patients}

The major limitation of this study was due to the small population size. Further clinical studies in larger populations are necessary to investigate the reliability of Doppler vortography in the assessment of diastolic dysfunction. A large spectrum of diastolic dysfunction including hypertensive patients must be considered to compare the potential clinical significance of Doppler vortography technique which is based on vortex imaging with common clinical parameters used for the assessment of ventricular diastolic function. The vortex formation time (VFT) could be also measured using the echo parameters described in section 2.1.4.1. In addition, the chamber quantification and diastolic and systolic functions such as LV dimensions, LV end diastolic volume, EF, mitral inflow pattern, E wave propagation velocity and velocity of mitral annular movement $\left(\mathrm{e}^{\prime}\right)$ during early diastole must be assessed. Moreover, mitral inflow propagation velocity $\left(\mathrm{V}_{\mathrm{p}}\right)$ must also be estimated using color M-mode Doppler (refer to section 1.2.1.1). In this prospective study two parameters must be evaluated using our vortex imaging tool: the presence or not of the apical vortex and the vorticity and size of the mitral and apical vortices. In addition to proposed vortex imaging parameters (core vorticity and position), chamber quantification as well as systolic and diastolic functions (i.e. $\mathrm{E} / \mathrm{A}, \mathrm{E} / \mathrm{V}_{\mathrm{p}}, \mathrm{E} / \mathrm{e}^{\prime} \ldots$ ) will be assessed to check if the proposed parameters can better predict occurrence of LV diastolic dysfunction.

\subsubsection{D Doppler vortography}

Three-dimensional echocardiography has emerged as an accurate, non-invasive and clinically compliant technique to quantify the left ventricular function and heart chamber volumes. However, it requires respiratory gated (breath hold) sequential imaging, or the use of a matrix phased array transducer [141]. It is also required that the cardiac rhythm and heart function stay stable during acquisition, therefore the spatial and/or temporal resolution of 3D ultrasound remain low which limits the image quality. Besides, 3D echocardiography is time consuming which constrains its routine clinical applications for diagnosis and assessment of 
the cardiac function. Recent advancements in ultrasound vastly ameliorated the temporal and spatial resolutions of ultrasound devices, and it is anticipated that the real-time high-resolution 3D echocardiography will become a widespread routine clinical practice in the near future $[142,143]$. It should be pointed out that, although Doppler vortography has focused on 2D echocardiographic vortex imaging, this technique may be upgraded to a three-dimensional ultrasound modality in future to potentially improve its diagnostic accuracy in clinical applications.

\subsubsection{Develop a user-friendly software}

Finally, it is required to improve Doppler vortography technique in order to prepare a complete and user-friendly software for Doppler vortex imaging (Doppler vortography) which will be vastly utilized in daily clinical practice. This software will be easily used by clinicians for a thorough quantification of intracardiac vortices and to improve the assessment of left ventricular diastolic function based on conventional Doppler images only. 


\section{CHAPTER 6 CONCLUSION}

In this research project, Doppler vortography as a new echocardiographic tool for fast-and-easy intra-ventricular vortex quantification in daily clinical practice has been validated. Numerical and experimental studies were performed to validate Doppler vortography technique and analyze the effects of the transducer position and the size of the kernel filter. In the next step, as a preliminary analysis Doppler vortography was tested in 18 subjects belonging to two different groups (9 normal and 9 hypertensive patients). The vorticity of the mitral anterior vortex was calculated and compared. Also Doppler vortography was used to test whether an extra apical vortex is present in case of cardiac dysfunction. The vortex measurements were performed by a blinded operator. It has been demonstrated that Doppler vortography can accurately estimate the core vorticities of mitral and apical vortices within the LV. Our preliminary results display the feasibility of the in silico, in vitro and clinical studies described in this study. According to our promising findings, it is very likely that Doppler vortex imaging by Doppler vortography could expand the diagnostic value of echocardiography. More importantly, Doppler vortex imaging has the potential to significantly improve the assessment of left ventricular diastolic function, which presently can remain very challenging. This interface is clinically compliant, user independent, and noninvasive. It can be easily implemented for routine checks to recognize ventricular insufficiency. Also Doppler vortography has the ability to identify abnormal blood patterns at early stages of heart failure to decrease the morbidity of cardiac disease. At this stage of the research study, our assertion regarding the presence of the additional vortex near the apex is hypothetical. It is essential to increase the population size for future work to enable stronger conclusions regarding the apical vortex. A prospective study on a larger population size will help us to better specify the potential clinical relevance of Doppler vortography in ventricular function especially in diastology. 


\section{BIBLIOGRAPHY}

[1] P. J. Kilner, G. Z. Yang, A. J. Wilkes, R. H. Mohiaddin, D. N. Firmin, and M. H. Yacoub, "Asymmetric redirection of flow through the heart," Nature, vol. 404, pp. 759-761, 2000.

[2] G. Pedrizzetti and F. Domenichini, "Nature optimizes the swirling flow in the human left ventricle," Physical review letters, vol. 95, p. 108101, 2005.

[3] A. J. Vander, J. H. Sherman, and D. S. Luciano, Human Physiology: The Mechanisms of Body Function, $9^{\text {th }}$ ed. Michigan: McGraw-Hill education, 2004.

[4] A. J. Vander, J. H. Sherman, and D. S. Luciano, Human Physiology: The Mechanisms of Body Function, $8^{\text {th }}$ ed. Michigan: McGraw-Hill education, 2001.

[5] R. E. Klabunde, Cardiovascular physiology concepts, $2^{\text {nd }}$ ed. New York: Lippincott Williams \& Wilkins, 2011.

[6] A. Pick. (2008). Heart Valve Surgery Resources for Patients \& Caregivers. Available: http://www.heart-valve-surgery.com/

[7] Z. G. Turi, "Mitral valve disease," Circulation, vol. 109, pp. e38-e41, 2004.

[8] "Statistics Canada. Morality, Summary List of Causes 2008. Released October 18, 2011."

[9] A. Alwan, T. Armstrong, M. Cowan, and L. Riley, "Noncommunicable Diseases: Country Profiles 2011," Geneva: World Health Organisation, 2011.

[10] P. Liu, M. Arnold, I. Belenkie, J. Howlett, V. Huckell, A. Ignazewski, M. H. LeBlanc, R. McKelvie, J. Niznick, and J. D. Parker, "The 2001 Canadian Cardiovascular Society Guideline Update for the Management and Prevention of Congestive Heart Failure."

[11] A. Wielgosz, M. Arango, L. Johansen, Y. Mao, C. Nair, G. Paradis, S. Phillips, S. Trevisanato, J. Tu, L. Turner, and E. Wilson, "The Growing Burden of Heart Disease and Stroke in Canada 2003," Heart and Stroke Foundation of Canada, Ottawa, Canada, 2003.2003.

[12] S. A. Hunt, W. T. Abraham, M. H. Chin, A. M. Feldman, G. S. Francis, T. G. Ganiats, M. Jessup, M. A. Konstam, D. M. Mancini, and K. Michl, "ACC/AHA 2005 guideline update for the diagnosis and management of chronic heart failure in the adult. A report of the American College of Cardiology/American Heart Association Task Force on Practice Guidelines (Writing Committee to Update the 2001 Guidelines for the Evaluation and Management of Heart Failure). Developed in collaboration with the American College of Chest Physicians and the International Society for 
Heart and Lung Transplantation. Endorsed by the Heart Rhythm Society," Circulation, p. CIRCULATIONAHA. 105.167586, 2005.

[13] "Conference Board of Canada. The Canadian Heart Health Strategy: Risk Factors and Future Cost Implications. Report February 2010."

[14] A. Mosterd and A. W. Hoes, "Clinical epidemiology of heart failure," Heart, vol. 93, pp. 1137-1146, 2007.

[15] S. F. Nagueh, C. P. Appleton, T. C. Gillebert, P. N. Marino, J. K. Oh, O. A. Smiseth, A. D. Waggoner, F. A. Flachskampf, P. A. Pellikka, and A. Evangelisa, "Recommendations for the evaluation of left ventricular diastolic function by echocardiography," European Journal of Echocardiography, vol. 10, pp. 165-193, 2009.

[16] K.-L. Chan and J. P. Veinot, "Assessment of Diastolic Function," in Anatomic Basis of Echocardiographic Diagnosis, $1^{\text {st }}$ ed London: SpringerVerlag, 2011, pp. 421-438.

[17] C. Satpathy, T. K. Mishra, R. Satpathy, H. K. Satpathy, and E. Barone, "Diagnosis and management of diastolic dysfunction and heart failure," American family physician, 2006.

[18] J. K. Oh, S.-J. Park, and S. F. Nagueh, "Established and novel clinical applications of diastolic function assessment by echocardiography," Circulation: Cardiovascular Imaging, vol. 4, pp. 444-455, 2011.

[19] L. Mandinov, F. R. Eberli, C. Seiler, and O. M. Hess, "Diastolic heart failure," Cardiovascular research, vol. 45, p. 813, 2000.

[20] S. F. Nagueh, K. J. Middleton, H. A. Kopelen, W. A. Zoghbi, and M. A. Quiñones, "Doppler tissue imaging: a noninvasive technique for evaluation of left ventricular relaxation and estimation of filling pressures," Journal of the American College of Cardiology, vol. 30, pp. 1527-1533, 1997.

[21] L. Rodriguez, M. Garcia, M. Ares, B. P. Griffin, S. Nakatani, and J. D. Thomas, "Assessment of mitral annular dynamics during diastole by Doppler tissue imaging: comparison with mitral Doppler inflow in subjects without heart disease and in patients with left ventricular hypertrophy," American heart journal, vol. 131, pp. 982-987, 1996.

[22] M. J. Garcia, M. A. Ares, C. Asher, L. Rodriguez, P. Vandervoort, and J. D. Thomas, "An index of early left ventricular filling that combined with pulsed Doppler peak E velocity may estimate capillary wedge pressure," Journal of the American College of Cardiology, vol. 29, pp. 448-454, 1997.

[23] E. A. Ashley and J. Niebauer, Cardiology explained. London: Remedica, 2004. 
[24] M. Markl, P. J. Kilner, and T. Ebbers, "Comprehensive 4D velocity mapping of the heart and great vessels by cardiovascular magnetic resonance," Journal of Cardiovascular Magnetic Resonance, vol. 13, pp. $1-22,2011$.

[25] T. Ebbers, "Flow imaging: cardiac applications of 3D cine phase-contrast MRI," Current Cardiovascular Imaging Reports, vol. 4, pp. 127-133, 2011.

[26] D. H. Evans, W. N. McDicken, R. Skidmore, and J. Woodcock, Doppler ultrasound: physics, Instrumentation and Clinical Applications., $1^{\text {st }}$ ed. New York: John Wiley \& Sons Inc, 1991.

[27] D. H. Evans and W. N. McDicken, Doppler Ultrasound: Physics, Instrumentation and Signal Processing, $2^{\text {nd }}$ ed. New York: John Wiley \& Sons Inc, 2000.

[28] G. York and Y. Kim, "Ultrasound Processing and Computing: Review and Future Directions," Annu. Rev. Biomed. Eng, vol. 1, pp. 559-588, 1999.

[29] J. T. Bushberg, J. A. Seibert, E. M. Leidholdt Jr, J. M. Boone, and E. J. Goldschmidt Jr, "The essential physics of medical imaging," Medical Physics, vol. 30, p. 1936, 2003.

[30] J. Jensen, "Medical ultrasound imaging," Progress in biophysics and molecular biology, vol. 93, p. 153, 2007.

[31] R. S. Cobbold, Foundations of biomedical ultrasound, $1^{\text {st }}$ ed. New York: Oxford University Press, Inc, 2007.

[32] F. L. Arbona, B. Khabiri, and J. A. Norton, "Introduction to ultrasound," in Ultrasound-guided regional anesthesia: a practical approach to peripheral nerve blocks and perineural catheters, $1^{\text {st }}$ ed New York: Cambridge University Press, 2011.

[33] K. K. Shung, Diagnostic ultrasound: Imaging and blood flow measurements, $1^{\text {st }}$ ed. Boca Raton: CRC press, 2005.

[34] P. N. Wells, "Ultrasonic imaging of the human body," Reports on Progress in Physics, vol. 62, p. 671, 1999.

[35] M. Halliwell, "A tutorial on ultrasonic physics and imaging techniques," Proceedings of the Institution of Mechanical Engineers, Part H: Journal of Engineering in Medicine, vol. 224, pp. 127-142, 2010.

[36] M. Á. G. Fernández and J. J. Gómez de Diego, "Transthoracic echocardiography," in The EAE textbook of echocardiography, ed: Oxford University Press, 2011.

[37] B. E. Bulwer and J. M. Rivero, "Blood flow hemodynamics, cardiac mechanics, and Doppler echocardiography," in The transthoracic 
examination: echocardiography pocket guide, $1^{\text {st }}$ ed Mississauga: Jones and Bartlett, 2011.

[38] J. A. Jensen, Estimation of blood velocities using ultrasound: a signal processing approach: Cambridge University Press, 1996.

[39] J. Kirkhorn, "Introduction to IQ-demodulation of RF-data," IFBT, NTNU, vol. $15,1999$.

[40] K. Ferrara and G. DeAngelis, "Color flow mapping," Ultrasound in medicine \& biology, vol. 23, pp. 321-345, 1997.

[41] S. Bjaerum, H. Torp, and K. Kristoffersen, "Clutter filter design for ultrasound color flow imaging," Ultrasonics, Ferroelectrics and Frequency Control, IEEE Transactions on, vol. 49, pp. 204-216, 2002.

[42] A. Kheradvar and G. Pedrizzetti, Vortex formation in the cardiovascular system. London: Springer, 2012.

[43] G. K. Batchelor, An introduction to fluid dynamics. Cambridge university press, 1967.

[44] R. Panton, Incompressible flow, 3rd ed.: Hoboken: Wiley, 1983.

[45] G.-R. Hong, G. Pedrizzetti, G. Tonti, P. Li, Z. Wei, J. K. Kim, A. Baweja, S. Liu, N. Chung, and H. Houle, "Characterization and Quantification of Vortex Flow in the Human Left Ventricle by Contrast Echocardiography Using Vector Particle Image Velocimetry," JACC: Cardiovascular Imaging, vol. 1, 2008.

[46] O. Rodevand, R. Bjornerheim, T. Edvardsen, O. A. Smiseth, and H. Ihlen, "Diastolic flow pattern in the normal left ventricle," Journal of the American Society of Echocardiography, vol. 12, pp. 500-507, 1999.

[47] J. C. del Álamo, A. L. Marsden, and J. C. Lasherasa, "Recent advances in the application of computational mechanics to the diagnosis and treatment of cardiovascular disease," Revista Española de Cardiología (English Edition), vol. 62, pp. 781-805, 2009.

[48] Y. Bazilevs, J. C. del Alamo, and J. D. Humphrey, "From imaging to prediction: emerging non-invasive methods in pediatric cardiology," Progress in Pediatric Cardiology, vol. 30, pp. 81-89, 2010.

[49] P. P. Sengupta, G. Pedrizzetti, P. J. Kilner, A. Kheradvar, T. Ebbers, G. Tonti, A. G. Fraser, and J. Narula, "Emerging Trends in CV Flow Visualization," JACC: Cardiovascular Imaging, vol. 5, pp. 305-316, 2012.

[50] A. Kheradvar, H. Houle, G. Pedrizzetti, G. Tonti, T. Belcik, M. Ashraf, J. R. Lindner, M. Gharib, and D. Sahn, "Echocardiographic particle image velocimetry: a novel technique for quantification of left ventricular blood vorticity pattern," Journal of the American Society of Echocardiography, vol. 23, pp. 86-94, 2010. 
[51] C. J. Carlhäll and A. Bolger, "Passing Strange: Flow in the Failing Ventricle," Circulation Heart Failure, vol. 3, pp. 326-331, 2010.

[52] A. Kheradvar, R. Assadi, A. Falahatpisheh, and P. P. Sengupta, "Assessment of Transmitral Vortex Formation in Patients with Diastolic Dysfunction," Journal of the American Society of Echocardiography, 2011.

[53] T. Ishizu, Y. Seo, T. Ishimitsu, K. Obara, N. Moriyama, S. Kawano, S. Watanabe, and I. Yamaguchi, "The wake of a large vortex is associated with intraventricular filling delay in impaired left ventricles with a pseudonormalized transmitral flow pattern," Echocardiography, vol. 23, pp. 369-375, 2006.

[54] L. Jacobs, M. Kotler, and W. Parry, "Flow patterns in dilated cardiomyopathy: a pulsed-wave and color flow Doppler study," Journal of the American Society of Echocardiography, vol. 3, pp. 294-302, 1990.

[55] W. C. Little, "Diastolic Dysfunction Beyond Distensibility Adverse Effects of Ventricular Dilatation," Circulation, vol. 112, pp. 2888-2890, 2005.

[56] A. Pasipoularides, M. Shu, A. Shah, A. Tucconi, and D. D. Glower, "RV instantaneous intraventricular diastolic pressure and velocity distributions in normal and volume overload awake dog disease models," American Journal of Physiology-Heart and Circulatory Physiology, vol. 285, pp. H1956-65, 2003.

[57] A. Pasipoularides, M. Shu, A. Shah, M. S. Womack, and D. D. Glower, "Diastolic right ventricular filling vortex in normal and volume overload states," American Journal of Physiology-Heart and Circulatory Physiology, vol. 284, pp. H1064-72, 2003.

[58] R. Yotti, J. Bermejo, J. C. Antoranz, M. M. Desco, C. Cortina, J. L. RojoÁlvarez, C. Allué, L. Martín, M. Moreno, and J. A. Serrano, "A noninvasive method for assessing impaired diastolic suction in patients with dilated cardiomyopathy," Circulation, vol. 112, pp. 2921-9, 2005.

[59] Y. Zhang, W. Wang, X. Liang, Y. Bazilevs, M.-C. Hsu, T. Kvamsdal, R. Brekken, and J. Isaksen, "High-fidelity tetrahedral mesh generation from medical imaging data for fluid-structure interaction analysis of cerebral aneurysms," Computer Modeling in Engineering and Sciences (CMES), vol. 42, pp. 131-50, 2009.

[60] A. F. Bolger, E. Heiberg, M. Karlsson, L. Wigström, J. Engvall, A. Sigfridsson, T. Ebbers, J.-P. E. Kvitting, C. J. Carlhäll, and B. Wranne, "Transit of blood flow through the human left ventricle mapped by cardiovascular magnetic resonance," Journal of Cardiovascular Magnetic Resonance, vol. 9, pp. 741-747, 2007. 
[61] J. Eriksson, C. J. Carlhall, P. Dyverfeldt, J. Engvall, A. F. Bolger, and T. Ebbers, "Semi-automatic quantification of 4D left ventricular blood flow," J Cardiovasc Magn Reson, vol. 12, 2010.

[62] S. Cimino, G. Pedrizzetti, G. Tonti, E. Canali, V. Petronilli, L. De Luca, C. Iacoboni, and L. Agati, "In vivo analysis of intraventricular fluid dynamics in healthy hearts," European Journal of Mechanics-B/Fluids, 2012.

[63] D. Garcia, J. C. del Álamo, D. Tanné, R. Yotti, C. Cortina, É. Bertrand, J. C. Antoranz, E. Pérez-David, R. Rieu, and F. Fernández-Avilés, "Twodimensional intraventricular flow mapping by digital processing conventional color-Doppler echocardiography images," Medical Imaging, IEEE Transactions on, vol. 29, pp. 1701-1713, 2010.

[64] D. Garcia, J. C. del Álamo, C. Cortina, R. Yotti, D. Tanné, É. Bertrand, M. A. García-Fernández, F. Fernández-Avilés, and J. Bermejo, "Full Intraventricular Flow Mapping by Cconventional Color-Doppler Echocardiography," Journal of Biomechanics, vol. 41, p. S151, 2008.

[65] C. J. Carlhäll and A. Bolger, "Passing Strange Flow in the Failing Ventricle," Circulation: Heart Failure, vol. 3, pp. 326-331, 2010.

[66] B. Baccani, F. Domenichini, G. Pedrizzetti, and G. Tonti, "Fluid dynamics of the left ventricular filling in dilated cardiomyopathy," Journal of biomechanics, vol. 35, pp. 665-671, 2002.

[67] P. P. Sengupta, R. Burke, B. K. Khandheria, and M. Belohlavek, "Following the flow in chambers," Heart Failure Clinics, vol. 4, pp. 325$332,2008$.

[68] X. Lefebvre, S. He, R. Levine, and A. Yoganathan, "Systolic anterior motion of the mitral valve in hypertrophic cardiomyopathy: an in vitro pulsatile flow study," The Journal of heart valve disease, vol. 4, p. 422, 1995.

[69] X. Lefebvre, A. Yoganathan, and R. Levine, "Insights from in-vitro flow visualization into the mechanism of systolic anterior motion of the mitral valve in hypertrophic cardiomyopathy under steady flow conditions," Journal of biomechanical engineering, vol. 114, pp. 406-13, 1992.

[70] P. Jiamsripong, A. M. Calleja, M. S. Alharthi, M. Dzsinich, E. M. McMahon, J. J. Heys, M. Milano, P. P. Sengupta, B. K. Khandheria, and M. Belohlavek, "Impact of acute moderate elevation in left ventricular afterload on diastolic transmitral flow efficiency: analysis by vortex formation time," Journal of the American Society of Echocardiography, vol. 22, pp. 427-431, 2009.

[71] F. Mehregan, S. Muth, L. Allard, G. Cloutier, and D. Garcia, "Doppler Vortography - Detection and Quantification of the Vortices in the Left 
Ventricle," presented at the IEEE International Ultrasonics Symposium Florida, USA, 2011.

[72] F. Domenichini, G. Pedrizzetti, and B. Baccani, "Three-dimensional filling flow into a model left ventricle," Journal of Fluid Mechanics, vol. 539, pp. 179-198, 2005.

[73] G. K. Batchelor, The theory of homogeneous turbulence. Cambridge (UK): Cambridge University Press, 1953.

[74] R. Faludi, M. Szulik, J. D'hooge, P. Herijgers, F. Rademakers, G. Pedrizzetti, and J. U. Voigt, "Left ventricular flow patterns in healthy subjects and patients with prosthetic mitral valves: An in vivo study using echocardiographic particle image velocimetry," The Journal of thoracic and cardiovascular surgery, vol. 139, pp. 1501-1510, 2010.

[75] J. T. Thomas, R. F. Kelly, S. J. Thomas, T. D. Stamos, K. Albasha, J. E. Parrillo, and J. E. Calvin, "Utility of history, physical examination, electrocardiogram, and chest radiograph for differentiating normal from decreased systolic function in patients with heart failure," American Journal of Medicine, vol. 112, pp. 437-445, 2002.

[76] M. Kasner, D. Westermann, P. Steendijk, R. Gaub, U. Wilkenshoff, K. Weitmann, W. Hoffmann, W. Poller, H. P. Schultheiss, and M. Pauschinger, "Utility of Doppler echocardiography and tissue Doppler imaging in the estimation of diastolic function in heart failure with normal ejection fraction," Circulation, vol. 116, pp. 637-647, 2007.

[77] M. S. Maurer, D. Spevack, D. Burkhoff, and I. Kronzon, "Diastolic dysfunction: Can it be diagnosed by Doppler echocardiography?," Journal of the American College of Cardiology, vol. 44, pp. 1543-1549, 2004.

[78] J. O. Dabiri and M. Gharib, "The role of optimal vortex formation in biological fluid transport," presented at the Proceedings of the Royal Society B, 2005.

[79] M. Gharib, E. Rambod, A. Kheradvar, D. J. Sahn, and J. O. Dabiri, "Optimal vortex formation as an index of cardiac health," National Academy of Sciences vol. 103, pp. 6305-6308, 2006.

[80] G. Nucifora, V. Delgado, M. Bertini, N. A. Marsan, N. R. Van de Veire, A. C. T. Ng, H. M. J. Siebelink, M. J. Schalij, E. R. Holman, and P. P. Sengupta, "Left ventricular muscle and fluid mechanics in acute myocardial infarction," The American journal of cardiology, vol. 106, pp. 1404-1409, 2010.

[81] V. Fuster, R. W. Alexander, R. A. O'Rourke, R. Roberts, S. B. King, and H. J. Wellens, Hurst's the Heart. New York: McGraw-Hill 2004.

[82] M. Gharib, E. Rambod, and K. Shariff, "A universal time scale for vortex ring formation," Journal of Fluid Mechanics, vol. 360, pp. 121-140, 1998. 
[83] J. O. DABIRI and M. GHARIB, "Starting flow through nozzles with temporally variable exit diameter," Journal of Fluid Mechanics, vol. 538, pp. 111-136, 2005.

[84] A. Kheradvar, M. Milano, and M. Gharib, "Correlation between vortex ring formation and mitral annulus dynamics during ventricular rapid filling," ASAIO Journal, vol. 53, pp. 8-16, 2007.

[85] K. K. Poh, L. C. Lee, L. Shen, E. Chong, Y. L. Tan, P. Chai, T. C. Yeo, and M. J. Wood, "Left ventricular fluid dynamics in heart failure: echocardiographic measurement and utilities of vortex formation time," European Heart Journal-Cardiovascular Imaging, vol. 13, pp. 385-393, 2012.

[86] M. Belohlavek, "Vortex formation time: an emerging echocardiographic index of left ventricular filling efficiency?," European Heart JournalCardiovascular Imaging, vol. 13, pp. 367-369, 2012.

[87] A. F. Leite-Moreira, J. Correia-Pinto, and T. C. Gillebert, "Afterload induced changes in myocardial relaxation A mechanism for diastolic dysfunction," Cardiovascular research, vol. 43, pp. 344-353, 1999.

[88] R. A. Nishimura and A. Jamil Tajik, "Evaluation of Diastolic Filling of Left Ventricle in Health and Disease: Doppler Echocardiography Is the Clinician's Rosetta Stone," Journal of the American College of Cardiology, vol. 30, pp. 8-18, 1997.

[89] P. R. Moran, "A flow velocity zeugmatographic interlace for NMR imaging in humans," Magnetic resonance imaging, vol. 1, pp. 197-203, 1982.

[90] N. J. Pelc, R. J. Herfkens, A. Shimakawa, and D. R. Enzmann, "Phase contrast cine magnetic resonance imaging," Magnetic Resonance Quarterly, vol. 7, p. 229, 1991.

[91] E. M. Haacke, R. W. Brown, M. Thompson, and R. Venkatesan, "Magnetic resonance imaging: physical principles and sequence design," Recherche, vol. 67, p. $02,1999$.

[92] J. Töger, M. Kanski, M. Carlsson, S. J. Kovács, G. Söderlind, H. Arheden, and E. Heiberg, "Vortex Ring Formation in the Left Ventricle of the Heart: Analysis by 4D Flow MRI and Lagrangian Coherent Structures," Annals of Biomedical Engineering, pp. 1-11, 2012.

[93] L. Wigström, T. Ebbers, A. Fyrenius, M. Karlsson, J. Engvall, B. Wranne, and A. F. Bolger, "Particle trace visualization of intracardiac flow using time-resolved 3D phase contrast MRI," Magnetic Resonance in Medicine, vol. 41, pp. 793-799, 1999.

[94] W. Y. Kim, P. G. Walker, E. M. Pedersen, J. K. Poulsen, S. Oyre, K. Houlind, and A. P. Yoganathan, "Left ventricular blood flow patterns in 
normal subjects: a quantitative analysis by three-dimensional magnetic resonance velocity mapping," Journal of the American College of Cardiology, vol. 26, pp. 224-238, 1995.

[95] T. Uejima, A. Koike, H. Sawada, T. Aizawa, S. Ohtsuki, M. Tanaka, T. Furukawa, and A. G. Fraser, "A new echocardiographic method for identifying vortex flow in the left ventricle: numerical validation," Ultrasound in medicine \& biology, vol. 36, pp. 772-788, 2010.

[96] K. Fox, R. Patel, G. Graham, J. Taylor, J. Stark, M. De Leval, and F. Macartney, "Multiple and single ventricular septal defect. A clinical and haemodynamic comparison," British heart journal, vol. 40, p. 141, 1978.

[97] L. Capineri, M. Scabia, and L. Masotti, "A Doppler system for dynamic vector velocity maps," Ultrasound in medicine \& biology, vol. 28, pp. 237248, 2002.

[98] B. Dunmire, K. Beach, M. Plett, and D. Strandness, "Cross-beam vector Doppler ultrasound for angle-independent velocity measurements," Ultrasound in medicine \& biology, vol. 26, pp. 1213-1235, 2000.

[99] O. D. Kripfgans, J. M. Rubin, A. L. Hall, and J. B. Fowlkes, "Vector Doppler imaging of a spinning disc ultrasound Doppler phantom," Ultrasound in medicine \& biology, vol. 32, pp. 1037-1046, 2006.

[100] M. Fox and W. Gardiner, "Three-dimensional Doppler velocimetry of flow jets," Biomedical Engineering, IEEE Transactions on, vol. 35, pp. 834841, 1988.

[101] M. Arigovindan, M. Suhling, C. Jansen, P. Hunziker, and M. Unser, "Full motion and flow field recovery from echo Doppler data," Medical Imaging, IEEE Transactions on Medical Imaging, vol. 26, pp. 31-45, 2007.

[102] J. Udesen, M. B. Nielsen, K. R. Nielsen, and J. A. Jensen, "Examples of in vivo blood vector velocity estimation," Ultrasound in medicine \& biology, vol. 33, pp. 541-548, 2007.

[103] L. Bohs, B. Geiman, M. Anderson, S. Gebhart, and G. Trahey, "Speckle tracking for multi-dimensional flow estimation," Ultrasonics, vol. 38, pp. 369-375, 2000.

[104] H. Kim, J. Hertzberg, and R. Shandas, "Echo PIV for flow field measurements in vivo," Biomed Sci Instrum, vol. 40, pp. 357-63, 2004.

[105] L.-C. Lin, Y.-L. Ho, S.-L. Kao, C.-C. Wu, C.-S. Liau, and Y.-T. Lee, "Power Doppler-derived speckle tracking image of intraventricular flow in patients with anterior myocardial infarction: correlation with left ventricular thrombosis," Ultrasound in medicine \& biology, vol. 26, pp. 341-346, 2000. 
[106] L. Liu, H. Zheng, L. Williams, F. Zhang, R. Wang, J. Hertzberg, and R. Shandas, "Development of a custom-designed echo particle image velocimetry system for multi-component hemodynamic measurements: system characterization and initial experimental results," Physics in medicine and biology, vol. 53, p. 1397, 2008.

[107] P. P. Sengupta, B. K. Khandheria, J. Korinek, A. Jahangir, S. Yoshifuku, I. Milosevic, and M. Belohlavek, "Left Ventricular Isovolumic Flow Sequence During Sinus and Paced Rhythms:: New Insights From Use of High-Resolution Doppler and Ultrasonic Digital Particle Imaging Velocimetry," Journal of the American College of Cardiology, vol. 49, pp. 899-908, 2007.

[108] H. Zheng, L. Liu, L. Williams, J. R. Hertzberg, C. Lanning, and R. Shandas, "Real time multicomponent echo particle image velocimetry technique for opaque flow imaging," Applied physics letters, vol. 88, pp. 261915-261915-3, 2006.

[109] C. Willert and M. Gharib, "Digital particle image velocimetry," Experiments in fluids, vol. 10, pp. 181-193, 1991.

[110] R. J. Adrian, "Particle-imaging techniques for experimental fluid mechanics," Annual review of fluid mechanics, vol. 23, pp. 261-304, 1991.

[111] M. Qian, L. Niu, Y. Wang, B. Jiang, Q. Jin, C. Jiang, and H. Zheng, "Measurement of flow velocity fields in small vessel-mimic phantoms and vessels of small animals using micro ultrasonic particle image velocimetry (micro-EPIV)," Physics in medicine and biology, vol. 55, p. 6069, 2010.

[112] T. Hölscher, J. Rodriguez-Rodriguez, W. G. Wilkening, and J. C. Lasheras, "Intraoperative brain ultrasound: A new approach to study flow dynamics in intracranial aneurysms," Ultrasound in medicine \& biology, vol. 32, pp. 1307-1313, 2006.

[113] H. Gao, P. Claus, M.-S. Amzulescu, I. Stankovic, J. D'hooge, and J.-U. Voigt, "How to optimize intracardiac blood flow tracking by echocardiographic particle image velocimetry? Exploring the influence of data acquisition using computer-generated data sets," European Heart Journal-Cardiovascular Imaging, vol. 13, pp. 490-499, 2012.

[114] P. Vennemann, R. Lindken, and J. Westerweel, "In vivo whole-field blood velocity measurement techniques," Experiments in fluids, vol. 42, pp. 495$511,2007$.

[115] S. Ohtsuki and M. Tanaka, "The flow velocity distribution from the Doppler information on a plane in three-dimensional flow," Journal of visualization, vol. 9, pp. 69-82, 2006.

[116] M. Alhama, J. Bermejo, R. Yotti, E. Perez-David, Y. Benito, A. GonzalezMansilla, C. P. del Villar, F. Fernandez-Aviles, and J. del Alamo, 
"Quantitative Assessment of Intraventricular Vorticity Using Conventional Color-Doppler Ultrasound. Head to Head Clinical Validation Against Phase-Contrast Magnetic Resonance Imaging.," Journal of the American College of Cardiology, vol. 59, p. E1128, 2012.

[117] R. Chen, B. W. Zhao, B. Wang, H. L. Tang, P. Li, M. Pan, and L. L. Xu, "Assessment of Left Ventricular Hemodynamics and Function of Patients with Uremia by Vortex Formation Using Vector Flow Mapping," Echocardiography, 2012.

[118] H. Zhang, J. Zhang, X. Zhu, L. Chen, L. Liu, Y. Duan, M. Yu, X. Zhou, T. Zhu, and M. Zhu, "The Left Ventricular Intracavitary Vortex during the Isovolumic Contraction Period as Detected by Vector Flow Mapping," Echocardiography, vol. 29, pp. 579-587, 2012.

[119] J. Lu, W. Li, Y. Zhong, A. Luo, S. Xie, and L. Yin, "Intuitive visualization and quantification of intraventricular convection in acute ischemic left ventricular failure during early diastole using color Doppler-based echocardiographic vector flow mapping," The International Journal of Cardiovascular Imaging (formerly Cardiac Imaging), vol. 28, pp. 10351047, 2012.

[120] M. Alhama, Y. Benito, J. Bermejo, R. Yotti, E. Perez-David, A. Barrio, C. Perez del Villar, A. Gonzalez Mansilla, F. Fernandez Aviles, and J. C. Del Alamo, "Intraventricular vorticity favors conservation of kinetic energy along the cardiac cycle: analysis in patients with dilated cardiomyopathy by post-processing color-doppler images," Bulletin of the American Physical Society, vol. 56, 2011.

[121] K. Biswas, Introduction To Fluid Mechanics and Fluid Machines. New Delhi: Tata McGraw-Hill education, 2008.

[122] M. Raffel, C. E. Willert, and J. Kompenhans, Particle image velocimetry: a practical guide. Berlin: Springer Verlag, 1998.

[123] J. A. Jensen, "Field: A program for simulating ultrasound systems," in $10^{\text {th }}$ Nordicbaltic conference on biomedical imaging 1996, pp. 351-353.

[124] J. A. Jensen and N. B. Svendsen, "Calculation of pressure fields from arbitrarily shaped, apodized, and excited ultrasound transducers," Ultrasonics, Ferroelectrics and Frequency Control, IEEE Transactions on, vol. 39, pp. 262-267, 1992.

[125] P. G. Saffman, Vortex dynamics. Cambridge, UK: Cambridge university press, 1995.

[126] C. Kasai, K. Namekawa, A. Koyano, and R. Omoto, "Real-time twodimensional blood flow imaging using an autocorrelation technique," IEEE Trans. Sonics Ultrason, vol. 32, pp. 458-464, 1985. 
[127] S. Muth, S. Dort, I. A. Sebag, M. J. Blais, and D. Garcia, "Unsupervised dealiasing and denoising of color-Doppler data," Medical Image Analysis, 2011.

[128] D. Garcia, "Robust smoothing of gridded data in one and higher dimensions with missing values," Computational Statistics \& Data Analysis, vol. 54, pp. 1167-1178, 2010.

[129] D. Tanné, E. Bertrand, L. Kadem, P. Pibarot, and R. Rieu, "Assessment of left heart and pulmonary circulation flow dynamics by a new pulsed mock circulatory system," Experiments in fluids, vol. 48, pp. 837-850, 2010.

[130] D. Tanné, "Déterminants hémodynamiques de l'hypertension pulmonaire et de la thromboembolie suite au remplacement valvulaire mitral," Laval University, 2008.

[131] WHO, "A global brief on Hypertension: silent killer, global public health crisis," World Health Day 2013.

[132] K. C. Stewart, J. C. Charonko, C. L. Niebel, W. C. Little, and P. P. Vlachos, "Left ventricular vortex formation is unaffected by diastolic impairment," American Journal of Physiology-Heart and Circulatory Physiology, vol. 303, pp. H1255-H1262, 2012.

[133] J. J. Charonko, R. Kumar, K. Stewart, W. C. Little, and P. P. Vlachos, "Vortices Formed on the Mitral Valve Tips Aid Normal Left Ventricular Filling," Annals of Biomedical Engineering, pp. 1-13, 2013.

[134] F. Mouret, L. Kadem, E. Bertrand, J. G. Dumesnil, P. Pibarot, and R. Rieu, "Mitral Prosthesis Opening and Flow Dynamics in a Model of Left Ventricle: An In Vitro Study on a Monoleaflet Mechanical Valve," Cardiovascular Engineering: An International Journal, vol. 5, 2005.

[135] M. Nakamura, S. Wada, T. Mikami, A. Kitabatake, T. Karino, and T. Yamaguchi, "Effect of flow disturbances remaining at the beginning of diastole on intraventricular diastolic flow and colour M-mode Doppler echocardiograms," Medical and Biological Engineering and Computing, vol. 42, pp. 509-515, 2004.

[136] F. Domenichini, G. Querzoli, A. Cenedese, and G. Pedrizzetti, "Combined experimental and numerical analysis of the flow structure into the left ventricle," Journal of biomechanics, vol. 40, pp. 1988-1994, 2007.

[137] R. B. Thompson and E. R. McVeigh, "Fast measurement of intracardiac pressure differences with 2D breath-hold phase-contrast MRI," Magnetic Resonance in Medicine, vol. 49, pp. 1056-1066, 2003.

[138] J. Eriksson, C. Carlhäll, P. Dyverfeldt, J. Engvall, A. F. Bolger, and T. Ebbers, "Semi-automatic quantification of 4D left ventricular blood flow," J Cardiovasc Magn Reson, vol. 12, 2010. 
[139] J. Eriksson, A. Bolger, T. Ebbers, and C. Carlhäll, "Four-dimensional blood flow-specific markers of LV dysfunction in dilated cardiomyopathy," European heart journal cardiovascular Imaging, 2012.

[140] A. Stalder, M. Russe, A. Frydrychowicz, J. Bock, J. Hennig, and M. Markl, "Quantitative 2D and 3D phase contrast MRI: optimized analysis of blood flow and vessel wall parameters," Magnetic Resonance in Medicine, vol. 60, pp. 1218-1231, 2008.

[141] B. J. Krenning, M. M. Voormolen, and J. R. Roelandt, "Assessment of left ventricular function by three-dimensional echocardiography," Cardiovasc Ultrasound, vol. 1, p. 12, 2003.

[142] J. Pemberton, X. Li, T. Karamlou, C. A. Sandquist, K. Thiele, I. Shen, R. M. Ungerleider, A. Kenny, and D. J. Sahn, "The use of live threedimensional Doppler echocardiography in the measurement of cardiac outputan in vivo animal study," Journal of the American College of Cardiology, vol. 45, pp. 433-438, 2005.

[143] R. De Simone, G. Glombitza, C. F. Vahl, H. Meinzer, and S. Hagl, "Threedimensional Doppler. Techniques and clinical applications," European heart journal, vol. 20, pp. 619-627, 1999.

[144] F. Mehregan, F. Tournoux, S. Muth, P. Pibarot, R. Rieu, G. Cloutier, and D. Garcia, "Doppler Vortography: A Color Doppler Approach to Quantification of Intraventricular Blood Flow Vortices," Ultrasound in medicine \& biology, 2013. 
Appendix 1 - Proceeding article [71] 


\title{
Doppler Vortography - Detection and Quantification of the Vortices in the Left Ventricle
}

\author{
Forough Mehregan, Stéphan Muth, Louise Allard, Guy Cloutier, and Damien Garcia
}

\begin{abstract}
It has been speculated that the natural swirling flow that occurs in the normal left ventricle (LV) during $L V$ filling is optimized in terms of fluid energy dissipation. In vivo findings also revealed the formation of additional counter-rotating vortices in the presence of cardiac disease. Such unnatural vortices may significantly impair the $L V$ function due to important kinetic dissipation. We propose a new method for quantification of intracardiac vorticity - Doppler vortography - based on conventional Doppler images only.

Doppler vortography relies on the centrosymmetric properties of the vortices. Such properties induce particular symmetries in the Doppler flow data which can be exploited to describe the vortices quantitatively. For this purpose, a kernel filter was developed to derive a parameter, the blood vortex signature (BVS), that allows detecting the main intracardiac vortices and estimating their core vorticities. The reliability of BVS measured by Doppler vortography was assessed in mock Doppler vortical fields and compared with the ground-truth vorticity mapping. Doppler vortography was also tested in healthy subjects and patients.

The simulation and in vitro results demonstrated that Doppler vortography is a highly reliable technique for the detection and quantification of the intraventricular vortices. The diastolic BVS measured in the echographic laboratory, at the end of the LV early filling, revealed the presence of retrograde vortices in patients with cardiac disease.

Doppler vortography is a promising echocardiographic tool for quantification of vortex flow in the left ventricle. Our findings suggest that Doppler vortography potentially has relevant clinical interest for the assessment of $\mathrm{LV}$ diastolic function.
\end{abstract}

Index terms - Blood vortex signature, Doppler vortography, Intracardiac vortices, Vorticity

\section{INTRODUCTION}

$\mathrm{W}$ ITHIN the left ventricle (LV) of a normal heart, diastolic filling is characterized by the formation of an asymmetric swirling motion [1]-[3]. A large diastolic vortex that forms adjacent to the anterior mitral valve leaflet swirls in the natural flow direction and then helps to redirect blood towards

This work was supported by the Canadian Institutes of Health Research (CIHR) under grant MOP-106465 and by the Heart and Stroke Foundation of Québec (HSFQ)

F. M., S. M. and D.G. (corresponding author) are with the RUBIC Research Unit of Biomechanics and Imaging in Cardiology, CRCHUM Research Centre, University of Montreal Hospital, Montreal, QC, Canada

G.C. and L. A. are with the LBUM - Laboratory of Biorheology and Medical Ultrasonics, CRCHUM - Research Centre, University of Montreal Hospital, Montreal, QC, Canada the outflow tract during systole. Accordingly, recent numerical, in vitro and in vivo observations suggest that these vortex patterns minimize the fluid energy dissipation and optimize the LV myocardial efficiency [1], [4]. Vortices that form during LV filling thus have specific geometries and locations which could be determinant factors of the heart function [5], [6]. In patients with abnormal heart function, like acute myocardial infarction, there is an impairment of the wall motion that may disturb the flow and thus affect the geometry of the diastolic vortices. In addition, retrograde (counter-rotating) apical vortices may appear in the apex in early stages of heart failure [7]. As a result of this abnormal vortex formation, an increase in fluid energy dissipation is expected to occur, which may result in a significant increased cardiac work and oxygen demand. Besides phase-contrast MRI, one technique has been used to quantify the vortices in the LV: optical flow carried out on B-mode frames (echo-PIV) [8], [9]. Echo-PIV requires continuous intravenous injection of contrast agent to acquire intensity images suited for the cross-correlation algorithm. Due to the unstable property of bubble aggregates in the LV, a complex fine-tuning of the contrast infusion before image acquisition is required, which seriously limits the application of echo-PIV in the clinical practice. Another recent method used to investigate vorticity is vector flow mapping (VFM) which estimates velocity vectors from color Doppler dataset [7], [10]. In this approach, two-dimensional assumptions are used to develop a 2-D vector field distribution by deducing the angular velocity components. The clinical potential of these promising methods are still under investigation.

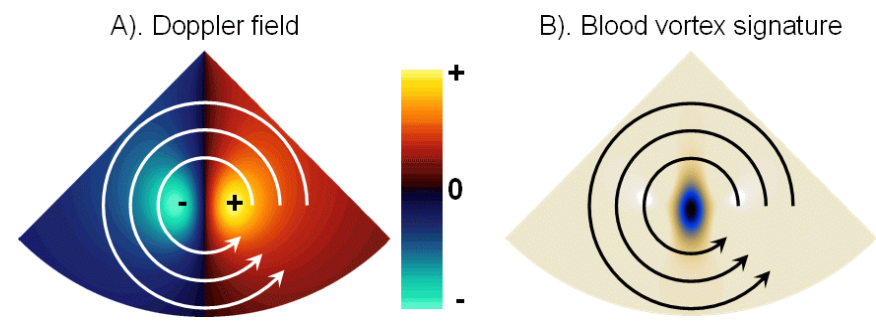

Fig. 1. A) Single large vortex (thick arrowed streamlines) and corresponding Doppler field; the phased-array transducer is at the top. B) Corresponding blood vortex signature (BVS); the BVS amplitude is maximal at the center of the vortex.

The objective of this study was to propose a new echocardiographic methodology, called Doppler vortography, for a thorough quantification of intracardiac vortices based on conventional Doppler images only. Based on symmetric properties observed in the Doppler field in the presence of vortices, we propose an index called "blood vortex signature" (BVS) obtained using a specific covariance-based kernel filter. This method can be easily implemented for routine checks to reco- 
gnize abnormal blood flow patterns at early stages of heart failure and to potentially provide an early marker of ventricular insufficiency. This manuscript mainly focuses on the validation of Doppler vortography and the estimation of the core vorticity by means of numerical and PIV (particle image velocimetry) data. Some preliminary results issued from healthy subjects and patients with heart disease are illustrated and discussed in the last section.

\section{METHOD}

In this section, we describe a new echocardiographic methodology - that we called Doppler vortography - for detecting and quantifying the vortices that form in the left ventricle during diastolic filling. Because this technique makes use of the conventional Doppler velocities only, Doppler vortography is fast, clinically-compliant and does not require any specific clinical adjustments. Assuming that the vortices are nearly axisymmetric, the vorticity - which describes the local rotational characteristics of the fluid - is estimated at the center of the detected vortices. In the following, since this manuscript focuses on ultrasound cardiac imaging only, the Doppler data are represented in a polar coordinate system $(r, \theta)$ whose origin is given by the central location of the phased-array transducer. In this configuration, the Doppler velocities thus represent the radial components of the velocity field.

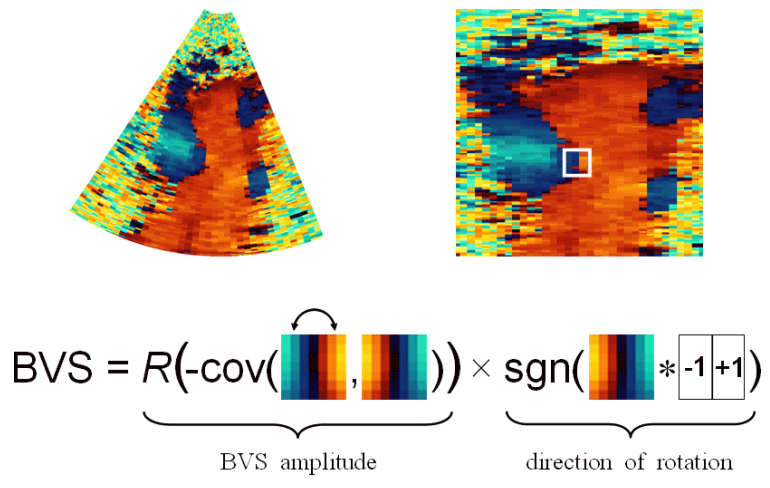

Fig. 2. Basic concept of the blood vortex signature (BVS). $R$ and sgn stand for the ramp and sign functions, respectively. Top panels: raw Doppler data in the Cartesian (left) and polar (right) coordinate systems.

\section{A. Blood vortex signature (BVS)}

By definition, a vortex is a fluid pattern that has a rapid swirling motion around its center. As a phased-array cardiac probe scans across the core of a vortex whose diameter is significantly larger than the ultrasound beam width, the Doppler velocities can provide a good representation of the velocity distribution across the vortex. Due to the centrosymmetric properties of a vortex, scanning such a flow pattern yields a scanline of zeroes surrounding by two maxima of opposite signs, as portrayed in Fig. 1A. Because of the nearly rotational symmetry of a vortex, it can also be noticed that flipping left to right a small kernel centered on the vortical core mostly modifies its sign only. Mathematically speaking, if $\left(r_{\mathrm{c}}, \theta_{\mathrm{c}}\right)$ denote the coordinates of the vortex center, the Doppler velocities $V_{D}$ at the vicinity of $\left(r_{\mathrm{c}}, \theta_{\mathrm{c}}\right)$ follow an odd function with respect to the angular component:

$$
V_{D}\left(r, \theta-\theta_{\mathrm{c}}\right)=-V_{D}\left(r, \theta_{\mathrm{c}}-\theta\right) \text {, if } r \approx r_{\mathrm{c}} \text { and } \theta \approx \theta_{\mathrm{c}} .
$$

Using this property, we can define the blood vortex signature (BVS). For this purpose, we now consider $V_{D}$ as a matrix array so that $V_{D \mathrm{ij}}=\left(r_{\mathrm{ij}}, \theta_{\mathrm{ij}}\right)$. Let $W_{\mathrm{ij}}$ represent a small window centered on $\left(r_{\mathrm{ij}}, \theta_{\mathrm{ij}}\right)$. The BVS parameter is given by:

$$
\mathrm{BVS}_{\mathrm{ij}}=\mathrm{R}\left(-\operatorname{cov}\left(W_{\mathrm{ij}}, \operatorname{fliplr}\left(W_{\mathrm{ij}}\right)\right)\right) \times S_{\mathrm{ij}},
$$

where $\mathrm{R}$ stands for the ramp function (i.e. $\mathrm{R}(\mathrm{x})=\mathrm{x}$, if $\mathrm{x} \geq 0 ;=0$ if $\mathrm{x}<0$ ) and "fliplr" represents a matrix operator that flips the columns in the left-right direction. The scalar $S_{\mathrm{ij}}(=1$ or -1$)$ reflects the direction (trigonometric vs. clockwise) of the vortex and can be easily calculated using a simple linear convolution. The BVS concept is depicted in Fig. 2. Due to the covariance measure in (2), the blood vortex signature is expected to have a high amplitude at the vicinity of a vortex and reaches a local extremum at its center. As an example, Fig. 1B illustrates the BVS related to the Doppler field represented in 1A. It can be noticed that the BVS amplitude is maximal at the core of the vortex.

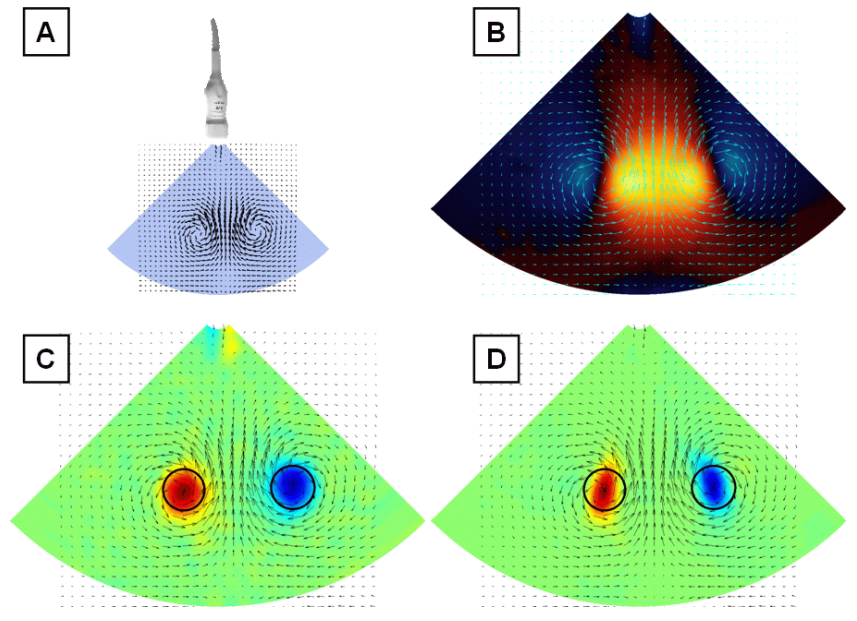

Fig. 3. A) Numerical model: a vortex pair is scanned with a cardiac probe. B) Corresponding Doppler velocities (without additive noise). C) Vorticity map (reference) calculated from the original vector flow field. 4) BVS map related to the Doppler field in 3.B.

\section{B. Estimation of the core vorticity}

As claimed in the previous paragraph, the BVS reaches a relative extremum at the center of a vortex. Measuring BVS by Doppler vortography may thus allow one to detect the vortical structures that form in the left ventricle during early filling. According to the recent literature devoted to the diastolic flow patterns, an accurate quantification of the intraventricular vortices could be of high clinical relevance, especially in acute myocardial infarction. A vortex can be mainly described by its core vorticity that is the vorticity at the center of the vortex, i.e. where the velocity vanishes. By definition, the vorticity in polar coordinates is given by:

$$
w=\frac{1}{r}\left(\frac{\partial}{\partial r}\left(r V_{\theta}\right)-\frac{\partial V_{r}}{\partial \theta}\right) .
$$

Because Doppler echocardiography provides the radial velocity component only (i.e. $V_{r}=-V_{D}$ ), the expression (3) is of no use in the clinical context. Assuming now that the vortex is nearly axisymmetric, one has, at the center of the vortex: 


$$
\frac{\partial}{\partial r}\left(r V_{\theta}\right)=-\frac{\partial V_{r}}{\partial \theta}, \text { for }(r, \theta)=\left(r_{\mathrm{c}}, \theta_{\mathrm{c}}\right) .
$$

Using (3) and (4), the core vorticity can be thus written as:

$$
w_{\mathrm{c}}=-\left.\frac{2}{r_{\mathrm{c}}} \frac{\partial V_{r}}{\partial \theta}\right|_{r_{\mathrm{c}}, \theta_{\mathrm{c}}}=\left.\frac{2}{r_{\mathrm{c}}} \frac{\partial V_{D}}{\partial \theta}\right|_{r_{\mathrm{c}}, \theta_{\mathrm{c}}} .
$$

\section{Numerical analyses using mock Doppler fields}

In order to quantify its accuracy, Doppler vortography was tested on $200 \times 64$ (i.e. 64 scanlines and 200 depth steps) mock Doppler fields with additive noise. The original Doppler velocity field $\left(V_{D}{ }^{0}\right)$ was obtained by zeroing the angular velocity components of a vortex pair (see Fig. 3A-B). $V_{D}{ }^{0}$ was corrupted by a zero-mean Gaussian white noise with a velocity-dependent local variance:

$$
V_{D}{ }^{\mathrm{n}}=V_{D}^{0}+\alpha \sqrt{\left|V_{D}{ }^{0}\right|} \mathrm{N}(0,1) \text {, }
$$

where $V_{D}{ }^{\mathrm{n}}$ represents the noisy Doppler velocity field, and $\alpha$ is a positive scalar that regulates the amount of noise. Thirty noisy configurations were tested, with $\alpha$ linearly spaced between $0(\mathrm{SNR}=+\infty)$ and $0.725(\mathrm{SNR}=3 \mathrm{~dB})$. Five hundred Doppler fields were simulated based on a Monte-Carlo method for each of these configurations. The BVS was calculated in all these corrupt fields using equation (2) with a kernel size of $15 \times 9$ (see Fig. 3D). The local extrema of the two main BVS patches (Fig. 3D) were detected and the core vorticities were estimated using equation (5) after a prior unsupervised smoothing of the noisy Doppler field [11]. The Doppler-derived core vorticities were compared with those estimated from the original vector flow field (Fig. 3C) using the normalized root mean squared error. The relative errors on the vortex positions were also calculated.

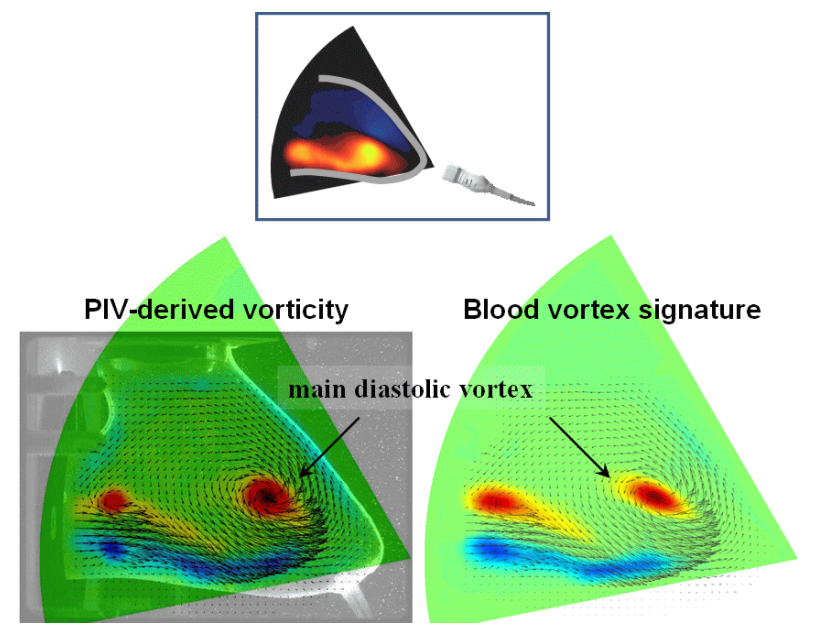

Fig. 4. PIV-derived vorticity vs. Blood vortex signature in a left heart model.

\section{In vitro analyses based on PIV experimentation in a left heart model}

In vitro experiments were performed using an atrioventricular dual activation pulse duplicator (ESIL, Marseilles, France). As previously described in [7], this in vitro model consists of ventricular and atrial activation boxes, systemic and pulmonary circulation models, and a computerized driving interface [12]. The left heart cavities are compliant and transparent. A liquid made of $40 \%$ glycerol and of $60 \%$ saline water was used as a blood substitute. The in vitro system was set to simulate 3 different hemodynamic conditions: heart rate (bpm) / stroke volume $(\mathrm{mL})=60 / 65,80 / 60$ and 100/75. The velocity field within the ventricular cavity was measured using particle image velocimetry (PIV). The laser plane cut the mitral and aortic valve planes as well as the apex to simulate an apical long-axis view. Neutrally-buoyant Nylon particles, with a mean diameter of 15-20 $\mu \mathrm{m}$ were used as markers. The image series were processed with a standard commercial software package (Insight3G, TSI Inc., Shoreview, MN) using interrogation windows of $64 \times 64$ pixels, providing a spatial resolution of $2 \times 2 \mathrm{~mm}^{2}$. Ensemble average velocity fields were obtained using 30 consecutive heart cycles. The PIV data were validated by means of an unsupervised regularizer [13] and the vorticity was estimated using the 8-point method [14] (see Fig. 4). Using these original PIV fields, $200 \times 64$ mock Doppler fields were simulated with the transducer located at the apical position (Fig. 4). As performed with the numerical simulations (see section I.C), the BVS was calculated using equation (2) with a kernel size of $15 \times 9$ (an example is given in Fig. 4). The local extremum of the BVS patch corresponding to the main diastolic vortex (see Fig. 4) was detected and the core vorticity was estimated using equation (5). The Doppler vortographyderived core vorticities (Eq. 5) were compared with those measured from the original PIV field (8-point method) using a standard linear regression. This analysis was performed on the frames related to diastolic filling only (total $=14$ frames).
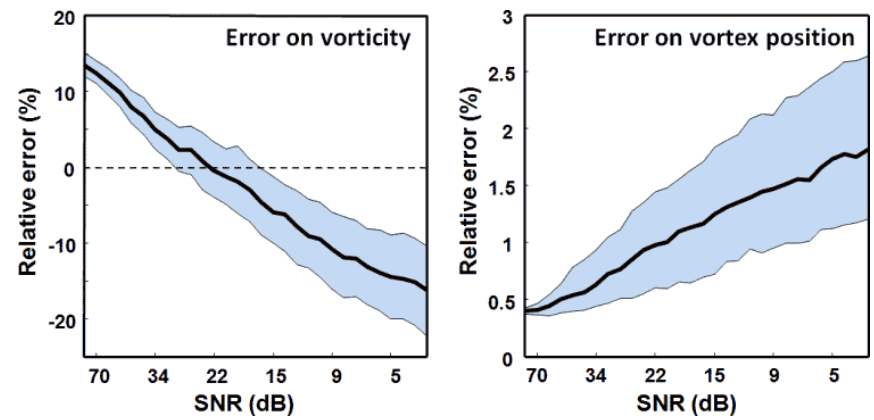

Fig. 5. Validation of Doppler vortography using mock Doppler fields in a vortex pair pattern. SNR stands for signal-to-noise ratio. The thick lines represent the median values. The gray areas delineate the 25th and 75 th percentiles. Left panel: errors on core vorticities estimated by Doppler vortography. Right panel: errors on vortex positions estimated from the BVS extrema.

\section{RESULTS}

The numerical analyses issued from the vortex pair (see section I.C) show that Doppler vortography (Eq. 5) slightly overestimated $(12 \%)$ the core vorticities without additive noise (Fig. 5, left). Due to some smoothing effect, this error decreased as the SNR decreased and significant underestimation appeared when the Doppler field became highly corrupted $(\mathrm{SNR}<10 \mathrm{~dB})$. As expected, the errors on vortex position as determined by the BVS extrema increased when the SNR decreased (Fig. 5, right). However, the vortex core detection by Doppler vortography remained highly accurate even with extremely noisy Doppler fields (errors $<2.5 \%$ ). The numerical 
analyses performed on an ideal vortex pair pattern thus show that Doppler vortography is robust to noise and may provide accurate detection (error $<5 \%$ ) and quantification (error $<$ $15 \%$ ) of the vortical structures. Regarding the mock Doppler fields issued from the PIV data, a high correlation was observed $\left(\rho=0.95, \mathrm{p}<10^{-6}\right)$ between the ground-truth PIV- and Doppler-derived core vorticities (i.e. 8-point method vs. Eq. 5, see Fig. 6). As with the numerical simulations (Fig. 5, left), a systematic overestimation, however, was observed (Fig 6). These results show that Doppler vortography can provide an accurate surrogate of core vorticity in the left ventricle.

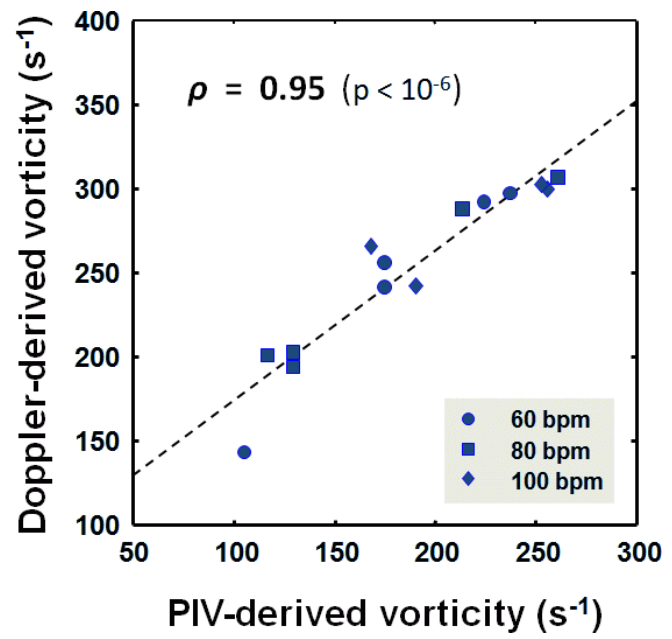

Fig. 6. Validation of Doppler vortography in an in vitro left heart model. The Doppler vortography-derived core vorticities were highly correlated with the PIV-derived vorticities ( $\rho$ is the Pearson's correlation coefficient, $n=14$ ).

\section{DISCUSSION AND PERSPECTIVES}

Detection and quantification of the left intraventricular vortices that emerge during early filling could be of high clinical interest in the study of LV function. Numerical results based on realistic mock Doppler fields clearly demonstrated that Doppler vortography can provide reliable measures of left ventricular vortices. As a very preliminary analysis, to test whether this technique is readily applicable in the clinical context, Doppler vortography was performed on five young healthy subjects using a GE Vivid E9 and on five patients with cardiac disease. BVS mapping revealed the presence of an anterograde vortex in contact with the anterior mitral valve leaflet ("A vortex", Fig. 7) at the end of the LV early filling both in healthy subjects and patients. This vortex was weaker in the patients with severe heart dysfunction (e.g. patient \#2, Fig. 7) probably due to an impaired left ventricular filling. The vortex adjacent to the posterior mitral valve leaflet was not always visible depending on the cross-sectional view. An atypical retrograde (counter-rotating) vortex was present in the apex of all of the patients (Fig. 7). Such an abnormal vortex very likely affects the left ventricular myocardial efficiency. We believe that the presence of an apical counter-rotating vortex, as revealed by Doppler vortography, should be an early marker of left ventricular dysfunction. A quantitative analy- sis of the anterior and apical vortices by estimating their vorticity and radius could help to better assess the heart function in the echocardiography laboratory.

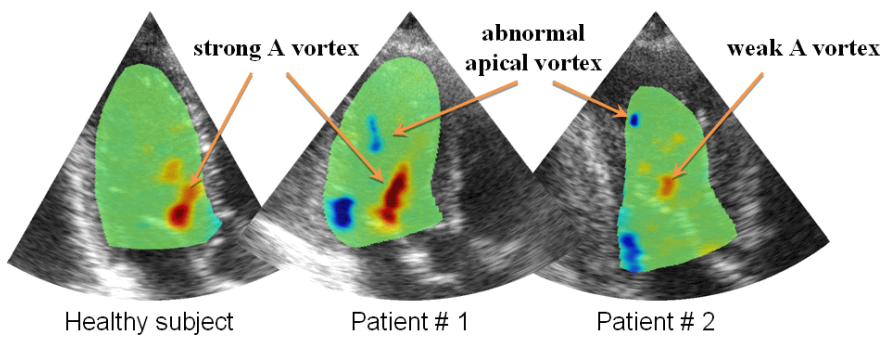

Fig. 7. Blood vortex signature measured by Doppler vortography in three subjects. "A vortex" means the vortex near the anterior mitral valve leaflet.

\section{REFERENCES}

[1] P. J. Kilner, G. Z. Yang, A. J. Wilkes, R. H. Mohiaddin, D. N. Firmin and M. H. Yacoub, "Asymmetric redirection of flow through the heart," Nature, vol. 404, pp. 759-761, Apr. 2000.

[2] O. Rodevand, R. Bjornerheim, T. Edvardsen, O. A. Smiseth and H. Ihlen, "Diastolic flow pattern in the normal left ventricle," J. Am. Soc. Echocardiogr., vol. 12, pp. 500-507, 1999.

[3] M. Gharib, E. Rambod, A. Kheradvar, D. J. Sahn and J. O. Dabiri, "Optimal vortex formation as an index of cardiac health," P. Natl. Acad. Sci. USA, vol. 103, no. 16, pp. 6305-6308, 2006.

[4] G. Pedrizzetti and F. Domenichini, "Nature optimizes the swirling flow in the human left ventricle," Phys. Rev. Lett., vol. 95, pp. 108101-1108101-4, 2005.

[5] G. Nucifora, V. Delgado, M. Bertini, N. A. Marsan, N. R. Van de Veire, A. C. T. Ng et al., "Left ventricular muscle and fluid mechanics in acute myocardial infarction," Am. J. Cardiol., vol. 106, pp. 1404-1409, 2010.

[6] G. R. Hong, G. Pedrizzetti, G. Tonti, P. Li, Z. Wei, J. K. Kim et al., "Characterization and quantification of vortex flow in the human left ventricle by contrast echocardiography using vector particle image velocimetry," J. Am. Coll. Cardiol. Img., vol. 1, pp. 705-717, 2008.

[7] D. Garcia, J. C. del Álamo, D. Tanné, R. Yotti, C. Cortina, E. Bertrand et al., "Two-dimensional intraventricular flow mapping by digital processing conventional color-Doppler echocardiography images," IEEE T. Med. Imaging, vol. 29, pp. 1701-1713, 2010.

[8] A. Kheradvar, H. Houle, G. Pedrizzetti, G. Tonti, T. Belcik, M. Ashraf et al., "Echocardiographic particle image velocimetry: a novel technique for quantification of left ventricular blood vorticity pattern," J. Am. Soc. Echocardiogr., vol. 23, pp. 86-94, 2010.

[9] P. Sengupta, B. K. Khandheria, J. Korinek, A. Jahangir, S. Yoshifuku, I. Milosevic et al., "Left ventricular isovolumic flow sequence during sinus and paced rhythms. New insights from use of high-resolution Doppler and ultrasonic digital particle image velocimetry," J. Am. Coll. Cardiol., vol. 49, no. 8, pp. 899-908, 2007.

[10] T. Uejima, A. Koike, H. Sawada, T. Aizawa, S. Ohtsuki, M. Tanaka et al., "A new echocardiographic method for identifying vortex flow in the left ventricle: numerical validation," Ultrasound Med. Biol., vol. 36, no. 5, pp. 772-788, 2010.

[11] S. Muth, S. Dort, I. A. Sebag, M. J. Blais, D. Garcia, "Unsupervised dealiasing and denoising of color-Doppler data," Med. Imag. Anal., vol. 15, pp. 577-588, 2011.

[12] D. Tanné, E. Bertrand, L. Kadem, P. Pibarot, R. Rieu, "Assessment of left heart and pulmonary circulation flow dynamics by a new pulsed mock circulatory system," Exp. Fluids, vol. 48, pp. 837-885, 2010.

[13] D. Garcia, "A fast all-in-one method for automated post-processing of PIV data," Exp. Fluids, vol. 50, pp. 1247-1259, 2011.

[14] M. Raffel, C. Willert, S. Wereley, J. Kompenhans, "Post-processing of PIV data," in Particle image velocimetry, 2nd ed., Springer, 2007, pp. 177-208. 
Appendix 2 - Journal article [144] 

Copyright (C) 2014 World Federation for Ultrasound in Medicine \& Biology Printed in the USA. All rights reserved $0301-5629 / \$$ - see front matter

\title{
DOPPLER VORTOGRAPHY: A COLOR DOPPLER APPROACH TO QUANTIFICATION OF INTRAVENTRICULAR BLOOD FLOW VORTICES
}

\author{
Forough Mehregan, ${ }^{* \dagger}$ François Tournoux, ${ }^{\ddagger}$ Stéphan Muth,${ }^{* \dagger}$ PhilipPe Pibarot, ${ }^{\S}$ Régis Rieu, \\ GuY Cloutier, ${ }^{\dagger \top \#}$ and Damien GarCia ${ }^{* \dagger \#}$ \\ * RUBIC, Research Unit of Biomechanics \& Imaging in Cardiology, University of Montreal Hospital, Montreal, QC, Canada; \\ ${ }^{\dagger}$ CRCHUM, Research Center, University of Montreal Hospital, Montreal, QC, Canada; ${ }^{\ddagger}$ Department of Echocardiography, \\ CHUM, University of Montreal Hospital, Montreal, QC, Canada; ${ }^{\S}$ Department of Medicine, Laval University and Québec Heart \\ \& Lung Institute, Laval University, Montreal, QC, Canada; "Aix-Marseille University, CNRS, UMR 7287, ISM, GIBOC, \\ Marseille, France; ${ }^{\uparrow}$ LBUM, Laboratory of Biorheology and Medical Ultrasonics, University of Montreal Hospital, Montreal, \\ QC, Canada; and \# Department of Radiology, Radio-Oncology and Nuclear Medicine and Institute of Biomedical Engineering, \\ University of Montreal, Montreal, QC, Canada
}

(Received 18 June 2013; revised 5 September 2013; in final form 9 September 2013)

\begin{abstract}
We propose a new approach to quantification of intracardiac vorticity based on conventional color Doppler images - Doppler vortography. Doppler vortography relies on the centrosymmetric properties of the vortices. Such properties induce particular symmetries in the Doppler flow data that can be exploited to describe the vortices quantitatively. For this purpose, a kernel filter was developed to derive a parameter, the blood vortex signature (BVS), that allows detection of the main intracardiac vortices and estimation of their core vorticities. The reliability of Doppler vortography was assessed in mock Doppler fields issued from simulations and in vitro data. Doppler vortography was also tested in patients and compared with vector flow mapping by echocardiography. Strong correlations were obtained between Doppler vortography-derived and ground-truth vorticities (in silico: $r^{2}=0.98$, in vitro: $r^{2}=0.86$, in vivo: $\left.r^{2}=0.89\right)$. Our results indicate that Doppler vortography is a potentially promising echocardiographic tool for quantification of vortex flow in the left ventricle. (C) 2014 World Federation for Ultrasound in Medicine \& Biology.
\end{abstract}

Key Words: Doppler echocardiography, Intraventricular blood flow, Vortex imaging, Vorticity, Doppler vortography, Vector flow mapping.

\section{INTRODUCTION}

Within the left ventricle of a normal heart, diastolic filling is characterized by the formation of a swirling motion during early filling (Gharib et al. 2006; Kilner et al. 2000; Toger et al. 2012): A large diastolic vortex forms adjacent to the anterior mitral valve leaflet and rotates in the natural flow direction. In the normal heart, a large part of the left ventricular (LV) blood volume is actually involved in the vortex formation. Because flowing blood keeps moving at the end of diastole, flow transition to ejection is ensured, which makes systole tightly coupled with diastolic filling (Carlhall and
Bolger 2010; Chan and Veinot 2011). Accordingly, recent in vitro and in vivo observations suggest that the normal vortex pattern might minimize the fluid energy dissipation and optimize LV myocardial efficiency (Charonko et al. 2013; Domenichini et al. 2007; Kilner et al. 2000; Pedrizzetti and Domenichini 2005). Vortices that form during LV filling thus have specific geometries and locations, which could be determinant factors of heart function (Hong et al. 2008; Nucifora et al. 2010). In patients with abnormal heart filling, there is an impairment of the wall/fluid dynamics that may disturb the flow patterns and, thus, affect the diastolic vortical structures (Nucifora et al. 2010). Reliable tools for imaging the intraventricular flow arrangements could be of major clinical interest for a better assessment of LV diastolic function. Comprehensive intracardiac velocity mapping can be measured by phase-contrast magnetic resonance imaging (MRI). 
Acquisition of 3-D cine phase contrast velocity data can indeed provide time-resolved characterization of blood flow in the left ventricle (Eriksson et al. 2010; Markl et al. 2011; Mohiaddin 1995; Toger et al. 2012; Wigstrom et al. 1999). MRI, however, is difficult to implement in routine practice because of limited accessibility and cost. Besides MRI, several echocardiographic techniques have been proposed in the last two decades to make vortex imaging more easily available for clinical daily practice. So far, four principal echocardiographic techniques have been described:

1. The earliest echographic studies regarding diastolic flow patterns are those based on observations from 2-D color Doppler and color M-mode echocardiography (Delemarre et al. 1990; Rodevand et al. 1999; Van Dantzig et al. 1995). Normal and abnormal flows were defined qualitatively according to the diastolic arrangements of the Doppler spectrum waveforms or of the red/blue-encoded Doppler velocities in the left ventricle. No quantitative measures of the vortices, however, were proposed.

2. Vortex formation time (VFT) has recently been proposed as an echocardiographic parameter to quantify the formation of LV vortices. VFT is a nondimensional index that characterizes the optimal conditions leading to vortex formation (Dabiri and Gharib 2005). It has also been claimed to be an index of cardiac function (Gharib et al. 2006). Recent clinical studies in acute cardiomyopathy have reported that VFT is reduced with impaired relaxation (Jiamsripong et al. 2009; Kheradvar et al. 2012; Nucifora et al. 2010; Poh et al. 2012). The VFT index, however, is simply a surrogate parameter that is calculated from standard echographic measures (stroke volume, mitral valve diameter, $\mathrm{E}$ and $\mathrm{A}$ waves). As a consequence, similarly to these standard parameters, it is expected that the VFT index may lack consistency in some situations. Whether this index is of clinical interest still remains questionable (Stewart et al. 2012).

3. Echo-particle image velocimetry (echo-PIV) is an efficient echographic tool for intraventricular velocity mapping (Cimino et al. 2012; Hong et al. 2008; Prinz et al. 2012). This technique, applied to contrast-enhanced echocardiographic images, is able to track ultrasound speckle displacements to estimate blood motion within the image plane (Gao et al. 2012; Kim et al. 2004). Recent studies have focused on LV vortex quantification by echo-PIV (Cimino et al. 2012; Faludi et al. 2010; Hong et al. 2008; Sengupta et al. 2012). This technique requires a continuous intravenous injection of contrast agent to reach an image quality suitable for motion tracking (Gao et al. 2012), seriously limiting the application of echo-PIV in daily clinical practice.

4. Cardiac Doppler vector flow mapping (VFM) is a technique based on 2-D color Doppler images and, thus, can be easily used for clinical applications. In the VFM approach, 2-D assumptions are used to develop an intracardiac vector distribution by deducing the velocity components perpendicular to the ultrasound beam within the entire Doppler field. Several techniques for intraventricular VFM have been recently been proposed (Arigovindan et al. 2007; Garcia et al. 2010; Uejima et al. 2010). Preliminary studies indicated promising results regarding the feasibility of LV vortex quantification in patients by VFM (Chen et al. 2012; Hendabadi et al. 2013; Lu et al. 2012; Zhang et al. 2012). Additional studies are still required to determine the accuracy and clinical reproducibility of Doppler VFM.

The new technique we propose-Doppler vortography-has been developed specifically to detect and quantify the intraventricular vortices that form during LV filling. Doppler vortography targets mainly particular local flow patterns present in the Doppler field using a fast detection algorithm. To detect and quantify the vortices, we propose an index called the "blood vortex signature" (BVS), obtained using a specific covariancebased kernel filter. This approach is thoroughly described in the next section. It is shown that Doppler vortography can estimate core vorticities accurately and that the results are concordant with those obtained by the vector flow mapping method.

\section{METHODS}

We here derive a new echocardiographic methodology, Doppler vortography, for detecting and quantifying the large-scale vortices that form in the left ventricle during diastolic filling. Figure 1 is a schematic example of vortex detection and quantification by Doppler vortography. As seen in this figure, for the particular case of a single large vortex, color Doppler exhibits an obvious antisymmetric imprint: negative mirror symmetry occurs with respect to the scan line crossing the vortex center. This anti-symmetry can also be used to detect intraventricular vortices. The Doppler vortography modality for intraventricular vortex imaging and the resulting BVS are described further below. In silico and in vitro studies were performed to validate the proposed technique and analyze the effects of transducer position and BVS filter kernel size. Doppler vortography was finally compared with VFM in patients using the VFM technique described by Garcia et al. (2010). 

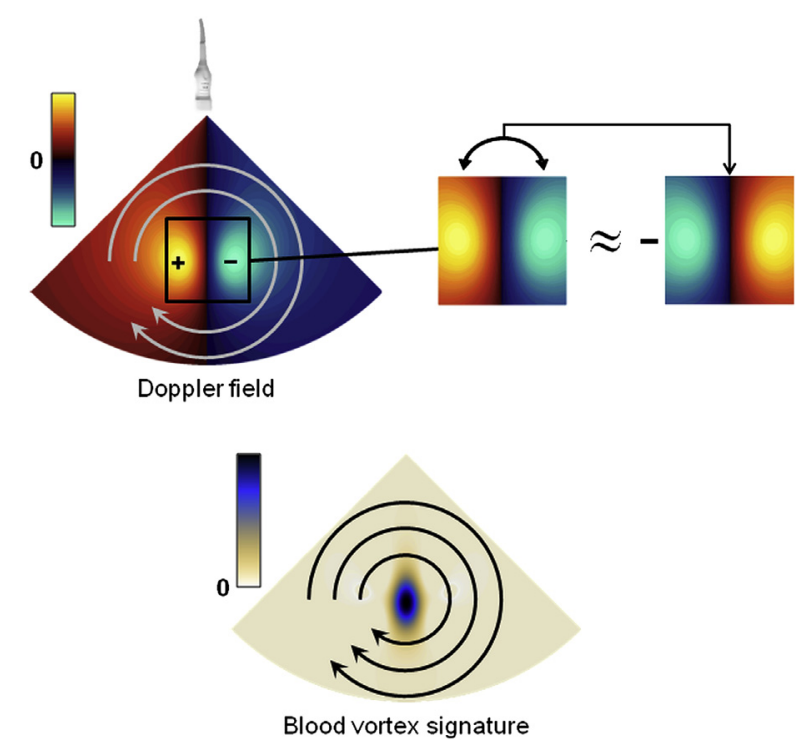

Fig. 1. Basic concept of Doppler vortography. For a single large vortex, color Doppler exhibits negative mirror symmetry with respect to the scan line crossing the vortex center (top). The proposed parameter, "blood vortex signature," allows detection of the vortex center from this antisymmetric property (bottom) (see also Fig. 2).

Assuming that the vortices are nearly axisymmetric around their center, the vorticity, which describes the local rotational characteristics of the fluid, is estimated at the core of the detected vortices. In the following, because we focus on ultrasound cardiac imaging, the Doppler data are represented in a polar coordinate system $(r, \theta)$ whose origin is related to the location of the phasedarray transducer. In this configuration, the Doppler velocities are thus given by the radial components of the velocity field.

\section{Theoretical concept of Doppler vortography}

A vortex is a particular flow arrangement that has a rapid swirling motion around its center. It is characterized mainly by its core vorticity, which somewhat reflects the "strength" of the spinning flow. As an ultrasound probe scans across a vortical flow whose scale is significantly larger than the ultrasound beam width, the Doppler field is characterized by a specific configuration that can be exploited to describe the vortex quantitatively: because of the centrosymmetric nature of a vortex, scanning such a flow pattern yields mainly a scan line of zeroes surrounded by two maxima of opposite signs (Fig. 1). Doppler vortography simply relies on this antisymmetric property. Because of the nearly rotational symmetry of a vortex, it is also noted that flipping left to right a small kernel centered on the vortical core mostly modifies its sign only (Fig. 1). Mathematically speaking, if $\left(r_{\mathrm{c}}, \theta_{\mathrm{c}}\right)$ denotes the polar coordinates of the vortex center, the Doppler velocities $V_{\mathrm{D}}$ about $\left(r_{\mathrm{c}}, \theta_{\mathrm{c}}\right)$ nearly follow an odd function with respect to the angular component:

$$
V_{\mathrm{D}}\left(r, \theta-\theta_{\mathrm{c}}\right) \approx-V_{D}\left(r,-\left(\theta-\theta_{\mathrm{c}}\right)\right) \quad \text { if } \quad r \approx r_{\mathrm{c}} \text { and } \theta \approx \theta_{\mathrm{c}} .
$$

To detect the locations $\left(r_{\mathrm{c}}, \theta_{\mathrm{c}}\right)$ of the vortex cores, one can seek the regions where the Doppler velocity follows eqn (1). For this purpose, we developed a simple kernel filter that returns a parameter (BVS) that reaches an extremum at a vortex core. We recall that we work on a regular polar grid $\left(r_{i}, \theta_{j}\right)_{i}=1 \ldots M, j=1 \ldots N$, where $M \times N$ is the size of the raw Doppler field (Fig. 2). Let $\mathbf{w}_{D}^{i j}$ represent a block of size $(2 m+1) \times(2 n+1)$ centered on $\left(r_{i}, \theta_{j}\right)$ and given by

$$
\mathbf{w}_{D}^{i j}=\left[V_{D}\left(r_{k}, \theta_{l}\right)\right]_{k=i-m \ldots i+m, l=j-n \ldots j+n} .
$$

The BVS parameter is now defined as (Fig. 2)

$$
\mathrm{BVS}_{i j}=\operatorname{BVS}\left(r_{i}, \theta_{j}\right)=R\left(\operatorname{cov}\left(\boldsymbol{w}_{D}^{i j},-\operatorname{fliplr}\left(\boldsymbol{w}_{D}^{i j}\right)\right)\right) \times S_{i j}
$$

where $R$ and cov stand for the ramp and covariance functions, respectively. The operator "fliplr" flips the matrix $\mathbf{w}_{D}^{i j}$ left to right, that is, in the angular direction. According to eqn (1), $\mathbf{w}_{D}^{i j} \approx-\operatorname{fliplr}\left(\mathbf{w}_{D}^{i j}\right)$ if the window $\mathbf{w}_{D}^{i j}$ is centered on a vortex core; the covariance operator allows one to detect where this equality occurs. The ramp function $R$ is used in eqn (3) because the negative covariance values are of no interest in this study. The rightmost term $S_{i j}$ in eqn (3) is a scalar ( $=1$ or -1$)$, which reflects the direction of the vortex (clockwise: 1, counterclockwise: -1 ). It is determined by using

$$
\left.S_{i j}=\operatorname{sgn} \sum_{k, l}\left(\mathbf{w}_{D}^{i j}(k, l-1)-\mathbf{w}_{D}^{i j}(k, l)\right)\right)
$$

where sgn is the signum function. Because of the covariance measure in eqn (3), the BVS yielded by Doppler vortography has high amplitude at the vicinity of a vortex and reaches a local extremum at its center (see an example in Fig. 3 based on a vortex pair). As such, the BVS parameter can help to detect the vortical structures that form in the left ventricle during early filling. It could also be of clinical interest to quantify them. A vortex is described mainly by its core vorticity. The vorticity $\omega$ in polar coordinates $(r, \theta)$ is given by the curl of the vector field

$$
\omega=\frac{1}{r}\left(\frac{\partial}{\partial r}\left(r V_{\theta}\right)-\frac{\partial V_{\mathrm{r}}}{\partial \theta}\right),
$$

where $V_{r}$ and $V_{\theta}$ are the radial and angular velocity components, respectively. The Doppler velocities $\left(V_{\mathrm{D}}\right)$ are related to the radial velocities as follows: $V_{\mathrm{r}}=-V_{\mathrm{D}}$. 


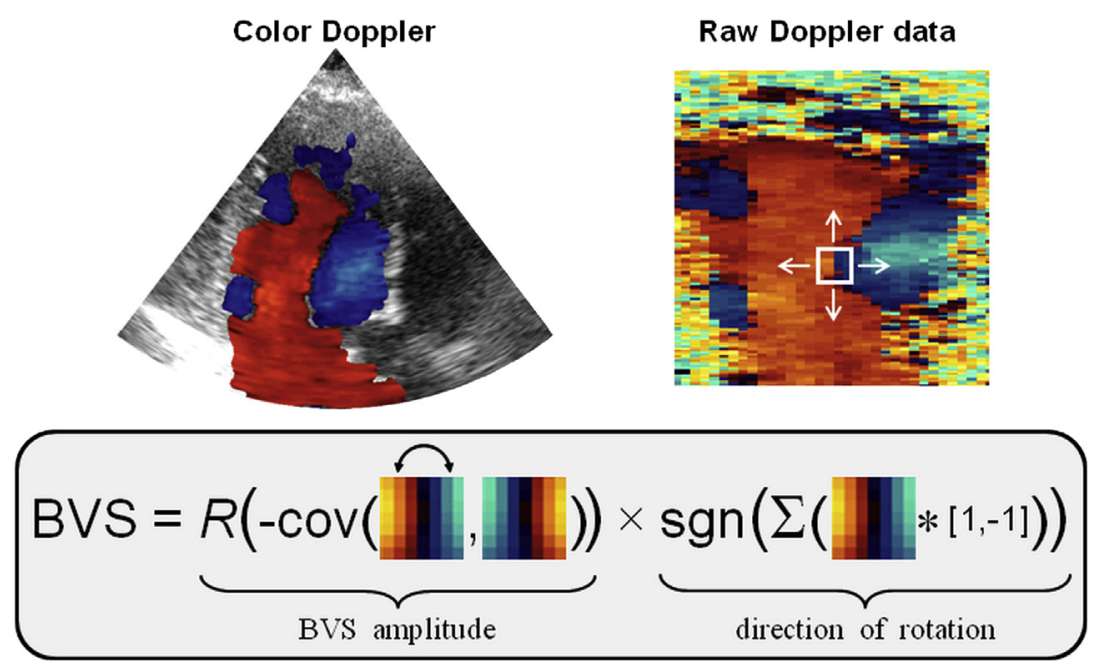

Fig. 2. Blood vortex signature. The blood vortex signature (BVS) is calculated using a small block sliding over the raw color Doppler array. The local BVS is based on the covariance (cov) between this block and its antisymmetric counterpart. The rotation direction is determined using a simple convolution. $R$ and sgn stand for the ramp and sign functions, respectively.

Assuming now that the vortex is axisymmetric at the vicinity of its core, one has, at the center of the vortex located at $\left(r_{\mathrm{c}}, \theta_{\mathrm{c}}\right)$,

$$
\frac{\partial}{\partial r}\left(r \quad V_{\theta}\right)=\frac{-\partial V_{\mathrm{r}}}{\partial \theta}, \quad \text { if } \quad(r, \theta)=\left(r_{\mathrm{c}}, \theta_{\mathrm{c}}\right)
$$

By use of eqns (5) and (6), the core vorticity $\omega_{\mathrm{C}}$ can finally be written as a function of the Doppler velocities:

$$
\omega_{\mathrm{c}}=\left.\left(\frac{-2}{r} \frac{\partial V_{r}}{\partial \theta}\right)\right|_{r_{\mathrm{c}}, \theta_{\mathrm{c}}}=\left.\frac{2}{r_{c}} \frac{\partial V_{\mathrm{D}}}{\partial \theta}\right|_{r_{\mathrm{c}}, \theta_{\mathrm{c}}}
$$

To sum up, Doppler vortography works as follows: (i) The BVS is measured during diastole from the color Doppler data using eqn (3). (ii) The main vortices are detected by seeking the extrema of BVS. (iii) Their core vorticities are estimated using eqn (7).
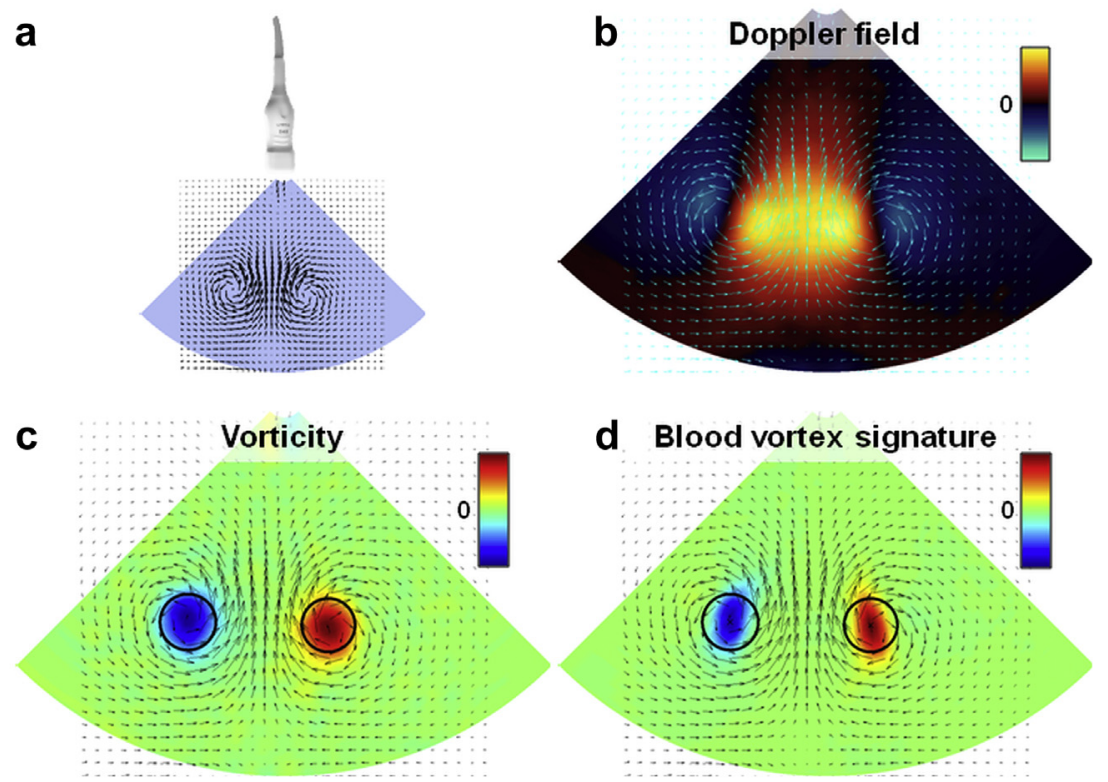

Fig. 3. Doppler vortography for a vortex pair. Insonification of a vortex pair (a) and corresponding mock color Doppler field (b). The "blood vortex signature" (BVS) parameter reaches an extremum at the vortex core (d). As a comparison, the ground-truth vorticity field is also depicted (c). 


\section{Numerical simulations}

To test the accuracy of Doppler vortography under ideal conditions, numerical ultrasound simulations were first performed with a Lamb-Oseen vortex. The LambOseen vortex is a commonly used vortex model in fluid dynamics. The circumferential velocity component $V_{\Phi}$ of this vortex is given by

$$
V_{\Phi}(\rho)=V_{\Phi_{\text {peak }}}\left(1+\frac{0.5}{\alpha}\right) \frac{\rho_{\mathrm{c}}}{\rho}\left(1-e^{-\alpha \frac{\rho^{2}}{\rho_{c}^{2}}}\right)
$$

where $\rho$ is the distance from the vortex center, $\alpha \approx 1.2564$, $V_{\Phi_{\text {peak }}}$ is the peak circumferential velocity and $\rho_{\mathrm{c}}$ is the core radius. From eqn (5), the core vorticity (at $\rho=0$ ) for a Lamb-Oseen vortex is deduced as

$$
\omega_{c}=\frac{2 \alpha+1}{\rho_{c}} V_{\Phi_{\text {peak }}}
$$

The ultrasound simulations were performed using the freeware Field II developed by Jensen and Svendsen (Jensen 1996; Jensen and Svendsen 1992). A 64element phased-array probe with a $0.3-\mathrm{mm}$ pitch was simulated. A total of 40,000 randomly positioned coplanar scatterers-ensuring fully developed speckles-were insonified at $2.5 \mathrm{MHz}$ with pulses containing eight cycles. The region of interest was $4 \mathrm{~cm}$ wide and ranged from 4 to $8 \mathrm{~cm}$ in depth respective to the probe. The Lamb-Oseen vortices were all centered within this region of interest. For a given vortex configuration, radiofrequency (RF) images containing 64 scan lines and covering a $40^{\circ}$ wide sector were created at a pulse repetition frequency of $7 \mathrm{kHz}$. The consecutive RF images were generated after the scatterers had been displaced according to the Lamb-Oseen velocity field. The Doppler signals were calculated from the demodulated RF images using a standard auto-correlator (Kasai et al. 1985) with a packet size of 5. A total of 31 vortex configurations were simulated. To generate these scenarios, the vortex core radius $\left(\rho_{\mathrm{c}}\right)$ was fixed at 1 ,
1.25 and $1.5 \mathrm{~cm}$, and the peak velocity $\left(V_{\Phi_{\text {peak }}}\right)$ ranged from 0.35 to $1.5 \mathrm{~m} / \mathrm{s}$. These values were chosen to be consistent with clinical observations (Garcia et al. 2010). The Doppler fields were de-aliased and smoothed using an unsupervised denoising method (Garcia 2010; Muth et al. 2011). For each Doppler field, the BVS was calculated from the simulated polar Doppler data using eqn (3), and the vortex core was located from the BVS extremum (Fig. 4). Several kernel sizes, that is, $(2 n+1) \times(2 n+1)$, where $n=1 \ldots 5$, were tested to analyze the robustness of the method. The effect of kernel size was analyzed using a repeated-measures analysis of variance (MedCalc Software, Version 12.5, Ostend, Belgium). The core vorticity was finally estimated using eqn (7) and compared with the ground-truth vorticity (eqn [9]) using a linear regression and a Bland-Altman plot.

\section{In vitro data}

The Doppler fields simulated with Field II were all based on perfectly axisymmetric vortices. Additional simulations were also performed with more realistic vortical patterns issued from in vitro data. These in vitro experiments were performed using an atrioventricular dual-activation pulse duplicator (courtesy of ESIL, Marseilles, France). As previously described in Garcia et al. (2010), this in vitro model consists of ventricular and atrial activation boxes, systemic and pulmonary circulation models and a computerized driving interface (Tanné et al. 2010). The left heart cavities are compliant and transparent. A liquid made of $40 \%$ glycerol and $60 \%$ saline water was used as a blood substitute. The in vitro system was set to simulate three different hemodynamic conditions: heart rate (bpm)/ stroke volume $(\mathrm{mL})=60 / 65,80 / 60$ and 100/75. The velocity field within the ventricular cavity was measured using optical laser-based PIV. The laser plane cut the mitral and aortic valve planes, as well as the apex, to simulate an apical three-chamber view. The image series was processed with a standard commercial software

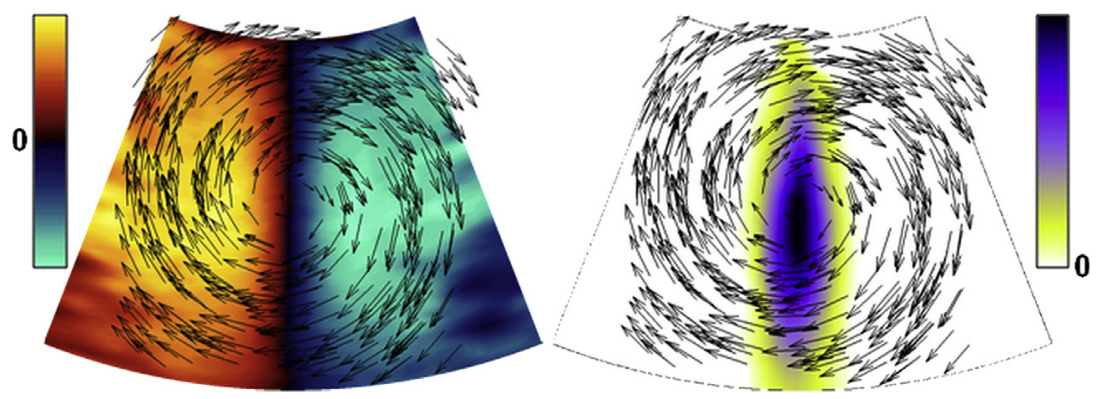

Fig. 4. In silico studies. Color Doppler fields were simulated in Lamb-Oseen vortices using Field II (left). The blood vortex signature was deduced from the color Doppler data using eqn (4) and allowed detection of the vortex core (right). The core vorticity yielded by Doppler vortography (eqn [7]) was compared with the theoretical core vorticity (eqn [9]) (see also Fig. 6). 
package (Insight3 G, TSI, Shoreview, MN, USA). Ensemble average velocity fields were obtained using 30 consecutive heart cycles. The PIV data were postprocessed by means of an unsupervised regularizer (Garcia 2011), and the vorticity was estimated using the eight-point method (Raffel et al. 2007). Through use of these PIV fields, $200 \times 64$ mock Doppler fields were simulated using Field II with the transducer located at the apical position. The parameters governing the simulations were similar to those reported in the previous subsection. The BVS was calculated using eqn (3) with different kernel sizes (an example is given in Fig. 5). The local extremum of the BVS patch corresponding to the main diastolic vortex (see Fig. 5) was detected, and the core vorticity was estimated using eqn (7). Doppler vortography-derived core vorticities (Eq. 7) were compared with those measured from the original PIV field (eight-point method) using standard linear regression and a Bland-Altman plot. They were both measured at the same location, that is, where the BVS amplitude was found to be maximal. This analysis was performed on the frames related to diastolic filling only (total $=14$ frames). To determine if transducer position affects the results returned by Doppler vortography, several probe angles relative to the left ventricle centroid were tested $\left(0^{\circ}, \pm 5^{\circ}, \pm 10^{\circ}\right)$ (see Fig. 5). The effects of kernel size and probe angle were analyzed independently using repeated-measures analyses of variance (MedCalc Software, Version 12.5, Ostend, Belgium).

\section{Clinical data}

The core vorticities obtained with the new Doppler vortography method were compared with those obtained by the vector flow mapping technique developed by Garcia et al. (2010). In the latter method, the crossbeam velocity components are deduced from the 2-D continuity equation with adequate boundary conditions (see Garcia et al. [2010] for details). Doppler echocardiographic images were acquired using a Vivid 7 ultrasound system (GE Healthcare, USA) in 19 patients (5 men, 14 women, mean age $=57 \pm 16 \mathrm{y}$ ). The use of these echographic images was approved by our local ethics committee. Informed consent was received from each participant. Among these patients, 12 were normotensive and 7 were hypertensive. They all had transthoracic echo considered as normal using common parameters. Apical three-chamber views with color Doppler over the entire left ventricular cavity were acquired during one to three cardiac cycles. The raw color Doppler data were exported from commercially available software (EchoPAC System, General Electric). A total of 55 frames were selected (average of 2.9 frames/patient). Only the frames obtained during early filling were considered in this study. Color Doppler data were analyzed using both Doppler vortography (eqns [3], [4] and [7]) and the vector flow mapping method proposed by Garcia et al. (2010). The core vorticity of the main mitral vortex (i.e., the vortex at the tip of the anterior mitral valve leaflet) was estimated by Doppler
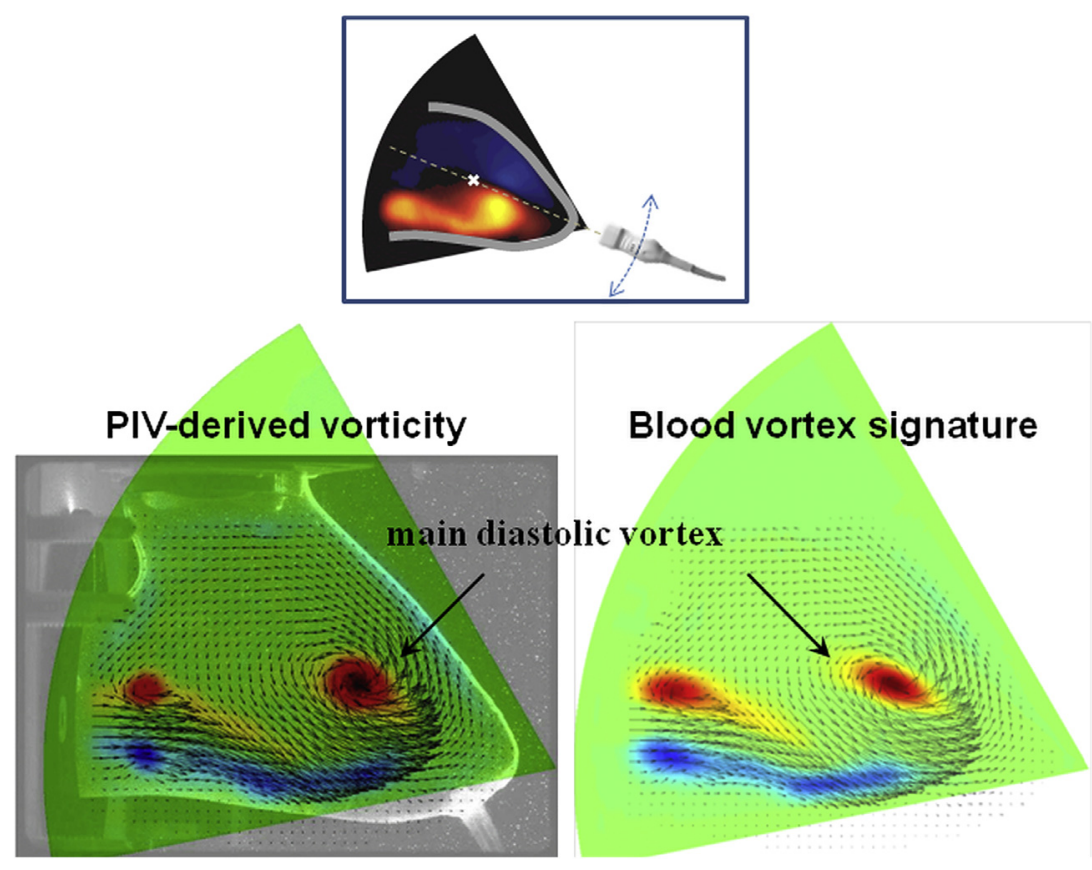

Fig. 5. In vitro data. Color Doppler fields were created from in vitro intraventricular particle image velocimetry (PIV) velocity fields. The blood vortex signature was measured by Doppler vortography, and the vorticity of the main diastolic vortex was compared with the PIV-derived vorticity. Several insonification angles were tested (see also Fig. 7). 
vortography as described under Theoretical Concept of Doppler Vortography. As a reference, the core vorticity of the same vortex was also calculated using the vector flow technique as previously developed by Garcia et al. (2010): the Okubo-Weiss criterion for vortex detection was derived from the vector velocity fields, and the corresponding core vorticities were determined. The OkuboWeiss criterion, also known as the $\mathrm{Q}$ criterion, is related to the second invariant of the velocity gradient tensor. Positive values point out the regions where vorticity prevails over strain rate (Hunt et al. 1988). Doppler vortography-derived core vorticities (eqn [7]) were compared with those measured by VFM using a standard linear regression and a Bland-Altman plot.

\section{RESULTS}

In silico and in vitro data: vortography-derived versus ground-truth vorticity

We observed good concordance between the vorticities estimated by Doppler vortography and those derived by control methods (in silico: analytical solution, in vitro: eight-point method). A strong correlation was obtained both in silico and in vitro $\left(r^{2}=0.98\right.$ and $\left.r^{2}=0.86\right)$ (see Figs. 6 and 7), with reasonably small relative errors in silico (Fig. 6, right). As revealed by the BlandAltman plot (Fig. 7, right), however, overestimations and higher relative errors were observed in vitro $(21 \pm 15 \%)$. This was due to the geometry of the vortices, whose cores were somewhat elliptic in the in vitro setup.

\section{In silico and in vitro data: effects of kernel size and} angle insonification

The repeated-measures analyses of variance revealed a significant effect of kernel size $(p<0.001)$, both in silico and in vitro. There was a negative linear trend $(p<0.0001)$ between kernel size and vorticity: that is, vorticity decreased with increasing kernel size. Pairwise comparisons, however, indicated that the mean vorticity differences between the $3 \times 3$ and $11 \times 11$ kernel configurations were $9.2 \pm 0.8 \mathrm{~s}^{-1}$ (in silico) and $12.3 \pm 2.1 \mathrm{~s}^{-1}$ (in vitro); these differences remained relatively small compared with the actual vorticity values (mean $>190 \mathrm{~s}^{-1}$ ). No significant effect of insonification angle $(p>0.05)$ was reported for the in vitro data. It can be concluded that kernel size and insonification angle had a negligible or no effect on the vorticity estimation.

\section{In vivo study: Doppler vortography versus VFM- derived vorticity}

Very good concordance between the vorticities estimated by Doppler vortography and VFM $(y=2.2+$ $\left.0.95 x, r^{2}=0.89, N=55, p<0.001\right)$ was observed in 19 patients (Fig. 8, left). The relative error between the two methods was $2.7 \pm 14 \%$ (Bland-Altman plot in Fig. 8). These findings indicate that good estimates of core vorticities can be obtained without the full vector components. To visually compare Doppler vortography and VFM, three examples are provided in Figure 9. The BVS maps returned by Doppler vortography (Fig. 9, second column) correlated well with the Okubo-Weiss criterion (Fig. 9, third column). Although these parameters are not aimed at being similar, they both allow vortex detection.

\section{DISCUSSION}

We have found that the echocardiographic tool that we derived, designated as Doppler vortography, can accurately detect and quantify the main intraventricular vortices that form during diastole. Intracardiac flow organization can be a robust marker of left ventricular filling. Blood flow is indeed very sensitive to its environment: as a fluid, and in contrast to solids, blood (on the macro scale) does not have a preferred shape because it does not possess any elastic behavior. In other words, a solid resists distortion, but fluid does not: contrary to solids, when a shear stress is applied to a fluid, the fluid continuously deforms. As a consequence, small dynamic and/or geometric perturbations may induce significant changes

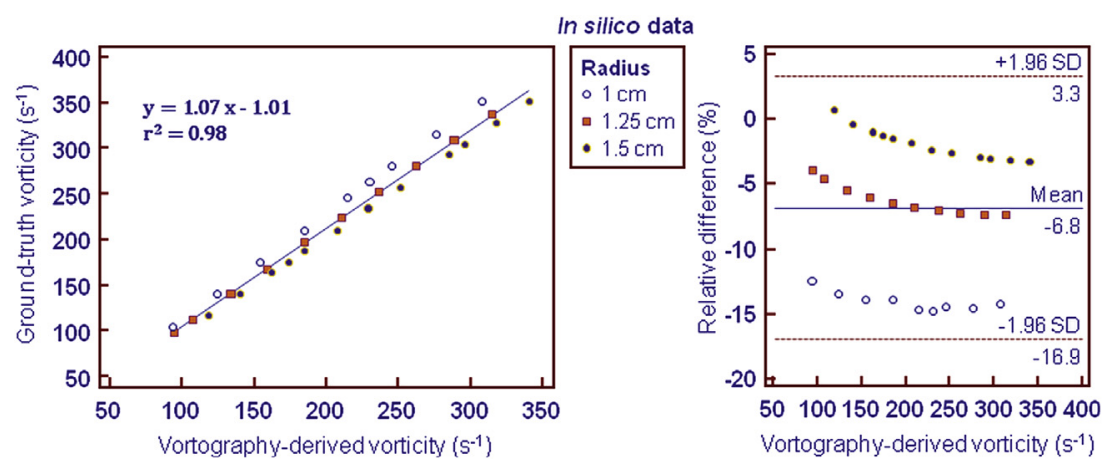

Fig. 6. In silico results. Comparison between vortography-derived (eqn [7]) and ground-truth (eqn [9]) vorticities. Different vortex core radii were simulated (see eqn [8]). SD = standard deviation. 


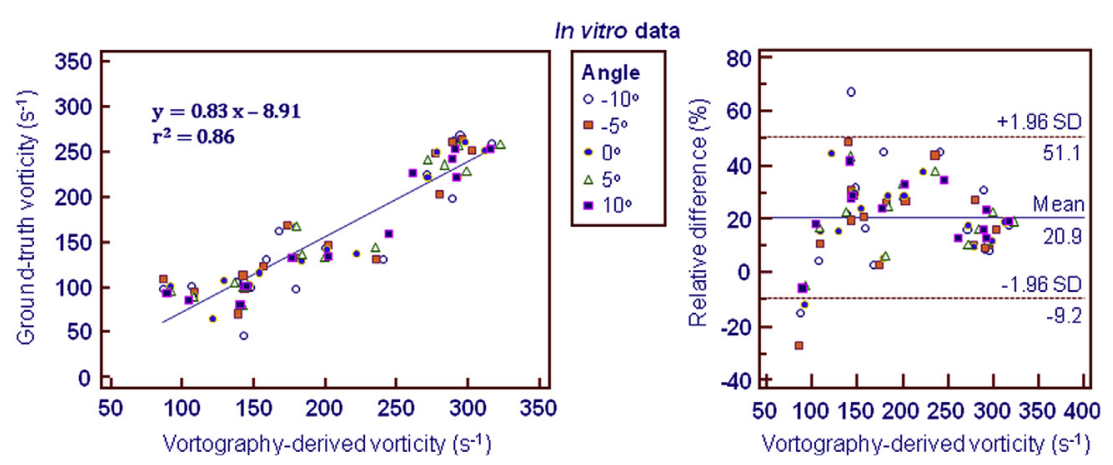

Fig. 7. In vitro results. Comparison between vortography-derived (eqn [7]) and ground-truth (eqn [9]) vorticities. Several angle insonifications were simulated (see Fig. 5). SD = standard deviation.

in flow patterns. For instance, in vascular flow, a slight modification in vessel geometry or flow dynamics, such as a bend, sharpness or acceleration, may modify blood flow patterns drastically. This phenomenon may be also true in the left ventricle and could be visible in the vortical structures (Kheradvar and Pedrizzetti 2012). It is thus anticipated that vortex analysis may provide additional information on left ventricular function. Concordantly, recent scientific literature reveals an emerging clinical interest in the characterization of intraventricular flow vortices (Belohlavek 2012; Cimino et al. 2012; Kheradvar et al. 2012; Poh et al. 2012; Toger et al. 2012). In this context, it was the aim of this study to propose a new color Doppler technique, Doppler vortography, for intraventricular vortex flow imaging. Because this technique makes use of conventional Doppler fields, Doppler vortography is fast, is clinically compliant and does not modify the clinical echo-lab routine.

\section{Why use the three-chamber view?}

In this study, we focused on the large vortical structures occurring in the apical long-axis three-chamber view. This plane passes through the apex and the centers of the mitral and aortic valves. Although intracardiac flow is known to be 3-D, phase-contrast MR studies have indicated that the out-of-plane velocity components are small in the plane corresponding to the echo three-chamber view (Eriksson et al. 2010, 2012; Wigstrom et al. 1999). Indeed, velocity data obtained by Wigstrom et al. (1999) and Eriksson et al. (2010, 2012) from 4-D phase contrast MR distinctly depict an intraventricular flow that is mainly parallel to the long-axis plane. In addition, clinically useful parameters of intraventricular flow dynamics can be obtained from a planar flow simplification using this view. In particular, Thompson and McVeigh (2003) reported that accurate pressure differences can be calculated using 2-D acquisitions because the momentum fluxes normal to the plane of interest are negligible. From these independent studies, it can be claimed that the utilization of 2-D statements in the particular three-chamber view is quite acceptable for analysis of the main flow vortices in most situations. We thus assumed that the main flow arrangements remain measurable in the three-chamber view, without significant loss of information. This assertion, however, may be invalid in severely diseased patients, in whom the 3-D nature of the flow could adversely influence Doppler vortography. Indeed, in such cases, the "anti-symmetry" hypothesis (see Fig. 1) could become ineffective. Further

\section{In vivo data}
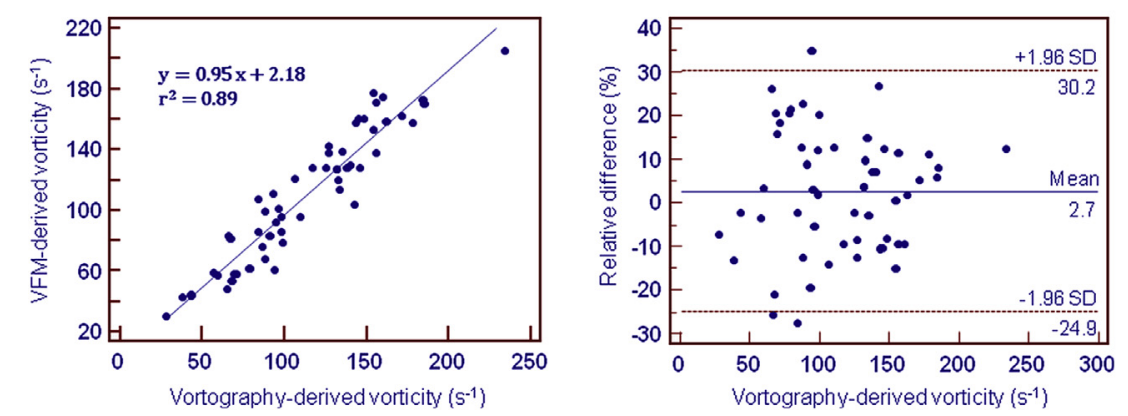

Fig. 8. In vivo results. Comparison between vortography-derived (eqn [7]) and vector flow mapping (VFM)-derived vorticities. Sample size $=55$ color Doppler frames in 19 patients. See Figure 9 for three examples. 

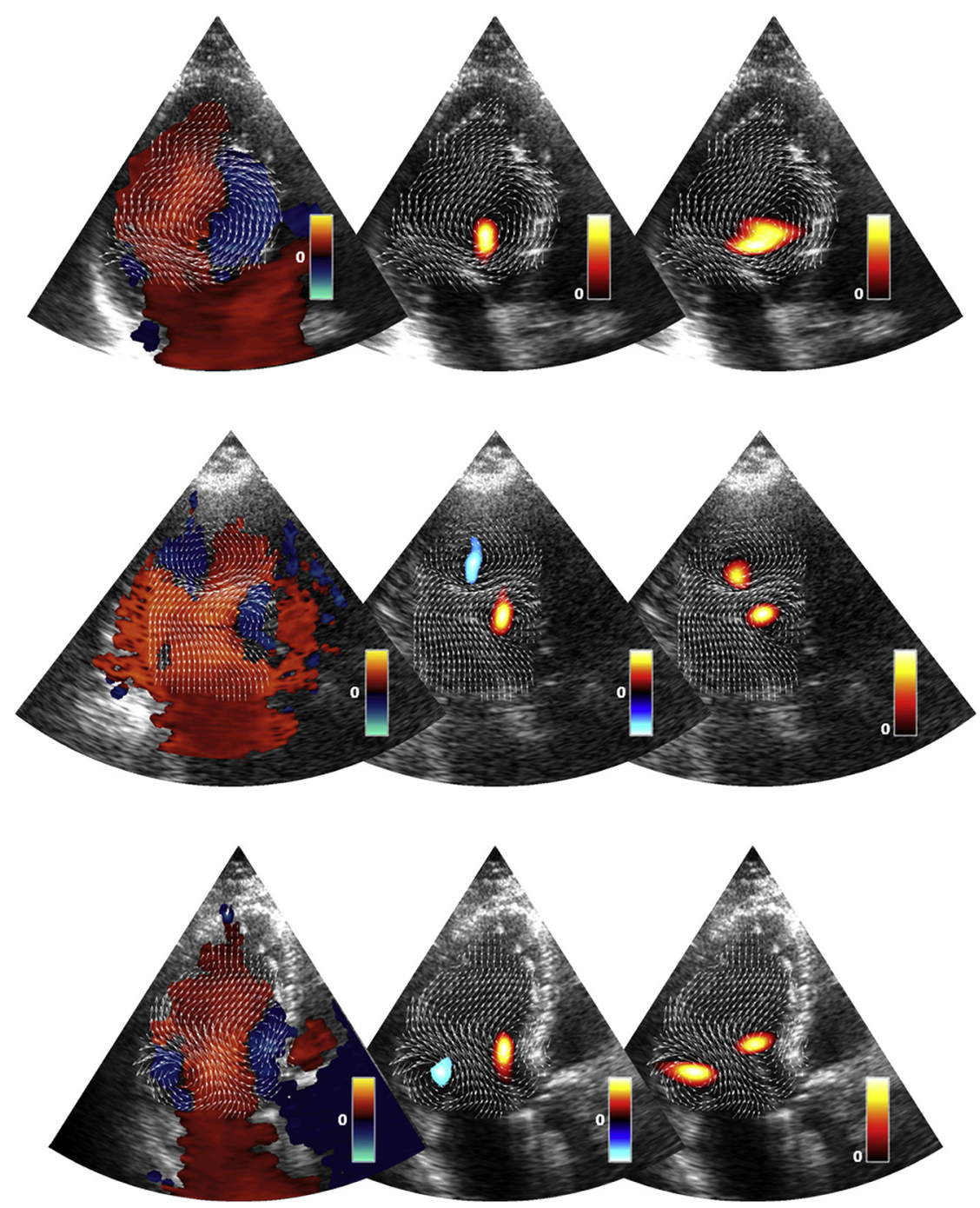

Fig. 9. Vector flow mapping (VFM) and Doppler vortography in three patients. The first column represents vector flows, as measured by the VFM technique described by Garcia et al. (2010), superimposed on conventional color Doppler images (the color bars represent Doppler velocities). In the second column are blood vortex signature (BVS) maps. High BVS amplitudes denote the presence of a large-scale vortex. The BVS sign represents the direction of rotation (see eqn [3]). The vector flows measured by VFM are also displayed for comparison purposes. The third column represents the Okubo-Weiss criterion derived from the VFM vector velocity fields. The Okubo-Weiss criterion is positive in zones of high vorticity. Note how well zones of high-amplitude BVS compare with this criterion. The images in the first row are from a normotensive subject with normal echo.

validation in mildly to severely diseased patients using velocimetry by cardiac magnetic resonance will help to better identify the physiologic conditions under which the 2-D hypothesis remains valid.

\section{Doppler vortography versus vector flow mapping}

Velocimetry by echo-PIV or phase-contrast MRI (see Introduction) would have been better gold standards than VFM, as these two techniques do not assume a planar flow. In contrast to the MRI and contrast echo approaches, however, Doppler vortography has the great advantage of being readily suitable for the clinical practice. Several techniques have been proposed for intracardiac vector flow mapping from conventional 2-D echocardiography, three of the most important ones being those described by Arigovindan et al. (2007), Garcia et al. (2010) and Uejima et al. (2010). The method proposed by Arigovindan et al. is not well adapted to cardiac flow imaging because two measurements differing by a significant angle are required. Although their theoretical concepts are different, the VFM approaches proposed by Uejima et al. and Garcia et al. are both based on single-color Doppler data set. The latter tactic minimizes the 2-D divergence to deduce the cross-beam velocity components 
and has been found to be an accurate quantitative method (Garcia et al. 2010); this is the VFM technique that was used in patients in our study. VFM, as Doppler vortography, is based on color Doppler data only. However, it uses a different numerical approach; this VFM method thus does not necessarily yield axisymmetric vortices. As depicted in Figure 8, we obtained very good concordance between Doppler vortography and VFM when determining the core vorticities in patients. When superimposing the velocity vectors yielded by VFM on the BVS map, it appeared that Doppler vortography was able to detect the main vortices adequately (see Fig. 9). From our simulations and in vivo data, it can be concluded that Doppler vortography is an efficient non-invasive method for detection and quantification of intraventricular vortices. Doppler vortography has the advantage of being numerically simpler and faster than VFM. More importantly, it directly targets the vortical flow patterns present in the color Doppler field using an original detection algorithm.

\section{Technical limitations of Doppler vortography}

Doppler vortography is based on 2-D color Doppler imaging and, thus, can be affected by the inherent Doppler artifacts, including aliasing, low signal-tonoise ratio, low angular and temporal resolutions and clutter signals (Mitchell 1990). The default clutter filter, as provided by the clinical GE ultrasound scanner, was used during the acquisitions in patients. It is likely that the cutoff frequency, within reasonable limits, little affected the results returned by Doppler vortography, because we were interested in the frames with relatively high velocities (E wave). A better alternative to suppress clutter signals, however, would be the use of efficient adaptive filters such as eigen-based clutter filters (Yu and Cobbold 2008; Yu and Lovstakken 2010). Furthermore, the Doppler signal-to-noise ratio was generally high in most cases during early filling. Low signal-to-noise ratio Doppler images were successfully de-aliased and denoised using the robust discrete cosine transform-based smoother (Muth et al. 2011). It should be noted that denoising does not influence vortex detection significantly because of the presence of a covariance-based filter, but it is required to obtain a consistent estimate of the core vorticity, as an angular derivative is necessary (see eqn [7]). Poor image sampling could also impede vortex detection/quantification. In all patients studied, however, the Doppler sectors contained at least 50 scan lines and enclosed the inner left ventricular cavity entirely to ensure high-quality measures. This was sometimes done at the expense of frame rate, but low frame rates do not affect the technique by itself. Of note, after some practice, the vortical regions can be spotted by an experienced eye from the color Doppler cine loops only. This actually helps to fine-tune the Doppler acquisition and to better adjust the Doppler sector. Finally, it should be pointed out that the pilot clinical study reported in this article was performed in only 19 patients. Because the echographic conditions encountered in these patients were all nearly optimal (no obesity, no cardiac complication, etc.), one must be aware that we likely missed clinical situations in which Doppler vortography may fail. A more exhaustive analysis performed in a large group of patients, with different echographic settings (pulse repetition frequency, signalto-noise ratio, wall filter, Nyquist velocity, etc.), would help to better identify the potential artifacts of Doppler vortography.

\section{Doppler vortography as a potential clinical tool for better assessment of diastolic function}

In some hypertensive patients (see Fig. 9, second row), a retrograde (i.e., counter-rotating with respect to the natural flow circulation) vortex was visible in the apex, by both Doppler vortography and VFM. In other patients (such as in Fig 9, first row, normotensive subject), a large vortex was present adjacent to the posterior mitral valve leaflet. Because a slight modification of the wall and/or inflow dynamics may significantly alter intraventricular flow, we believe that vortex flow imaging by Doppler vortography could be of help in the evaluation of diastolic function. In the presence of an apical vortex, the left ventricle may enter into a vicious cycle. Because it rotates in a direction opposite that of natural flow, the apical vortex induces excessive fluid energy dissipation and, thus, increases the workload. This may further disturb the blood flow filling patterns, which, in turn, decreases the vorticity of the main vortex. It could be expected that the vorticity of the major vortex is, to some extent, negatively related to the degree of filling impairment. At this stage of the study, this assertion remains hypothetical. A prospective study in a large group of patients is necessary to determine the potential clinical interest of Doppler vortography for the assessment of diastolic function and the prediction of adverse events.

\section{CONCLUSIONS}

Doppler vortography is able to decipher the vortical structures that form in the left ventricle during diastole. This color Doppler approach may offer new echographic insights into left ventricular function, especially for diastology.

Acknowledgments-This work was supported by operating grants from the Canadian Institutes of Health Research (CIHR, MOP-106465) and the Heart and Stroke Foundation of Québec (HSFQ). Damien Garcia holds a research scholarship award from the Fonds de Recherche en Santé du Québec (FRSQ). 


\section{REFERENCES}

Arigovindan M, Suhling M, Jansen C, Hunziker P, Unser M. Full motion and flow field recovery from echo Doppler data. IEEE Trans Med Imaging 2007;26:31-45.

Belohlavek M. Vortex formation time: An emerging echocardiographic index of left ventricular filling efficiency? Eur Heart J Cardiovasc Imaging 2012;13:367-369.

Carlhall CJ, Bolger A. Passing strange: Flow in the failing ventricle. Circ Heart Failure 2010;3:326-331.

Chan KL, Veinot JP. Assessment of diastolic function. In: Chan KL, (ed). Anatomic basis of echocardiographic diagnosis. London: Springer-Verlag; 2011. p. 421-438.

Charonko JJ, Kumar R, Stewart K, Little WC, Vlachos PP. Vortices formed on the mitral valve tips aid normal left ventricular filling. Ann Biomed Eng 2013;41:1049-1061.

Chen R, Zhao BW, Wang B, Tang HL, Li P, Pan M, Xu LL. Assessment of left ventricular hemodynamics and function of patients with uremia by vortex formation using vector flow mapping. Echocardiography 2012;29:1081-1090.

Cimino S, Pedrizzetti G, Tonti G, Canali E, Petronilli V, De Luca L, Iacoboni C, Agati L. In vivo analysis of intraventricular fluid dynamics in healthy hearts. Eur J Mech B/Fluids 2012;35:40-46.

Dabiri JO, Gharib M. The role of optimal vortex formation in biological fluid transport. Proc R Soc B 2005;272:1557-1560.

Delemarre BJ, Visser CA, Bot H, Dunning AJ. Prediction of apical thrombus formation in acute myocardial infarction based on left ventricular spatial flow pattern. J Am Coll Cardiol 1990;15: 355-360.

Domenichini F, Querzoli G, Cenedese A, Pedrizzetti G. Combined experimental and numerical analysis of the flow structure into the left ventricle. J Biomech 2007;40:1988-1994.

Eriksson J, Bolger AF, Ebbers T, Carlhall CJ. Four-dimensional blood flow-specific markers of LV dysfunction in dilated cardiomyopathy. Eur Heart J Cardiovasc Imaging 2012;14:417-424.

Eriksson J, Carlhall CJ, Dyverfeldt P, Engvall J, Bolger AF, Ebbers T. Semi-automatic quantification of 4-D left ventricular blood flow. J Cardiovasc Magn Reson 2010;12:9.

Faludi R, Szulik M, D'hooge J, Herijgers P, Rademakers F, Pedrizzetti G, Voigt JU. Left ventricular flow patterns in healthy subjects and patients with prosthetic mitral valves: An in vivo study using echocardiographic particle image velocimetry. J Thorac Cardiovasc Surg 2010;139:1501-1510.

Gao H, Claus P, Amzulescu MS, Stankovic I, D'hooge J, Voigt JU. How to optimize intracardiac blood flow tracking by echocardiographic particle image velocimetry? Exploring the influence of data acquisition using computer-generated data sets. Eur Heart J Cardiovasc Imaging 2012;13:490-499.

Garcia D. Robust smoothing of gridded data in one and higher dimensions with missing values. Comput Statist Data Anal 2010;54: $1167-1178$.

Garcia D. Fast all-in-one method for automated post-processing of PIV data. Experiments Fluids 2011;50:1247-1259.

Garcia D, del Álamo JC, Tanné D, Yotti R, Cortina C, Bertrand E, Antoranz JC, Rieu R, Garcia-Fernandez MA, Fernandez-Aviles F, Bermejo J. Two-dimensional intraventricular flow mapping by digital processing conventional color-Doppler echocardiography images. IEEE Trans Med Imaging 2010;29:1701-1713.

Gharib M, Rambod E, Kheradvar A, Sahn DJ, Dabiri JO. Optimal vortex formation as an index of cardiac health. Proc Natl Acad Sci USA 2006;103:6305-6308.

Hendabadi S, Bermejo J, Benito Y, Yotti R, Fernandez-Aviles F, del Álamo JC, Shadden SC. Topology of blood transport in the human left ventricle by novel processing of Doppler echocardiography. Ann Biomed Eng 2013 July 2 [EPub ahead of print].

Hong GR, Pedrizzetti G, Tonti G, Li P, Wei Z, Kim JK, Baweja A, Liu S, Chung N, Houle H, Narula J, Vannan MA. Characterization and quantification of vortex flow in the human left ventricle by contrast echocardiography using vector particle image velocimetry. JACC Cardiovasc Imaging 2008;1:705-717.
Hunt JCR, Wray AA, Moin P. Eddies, streams, and convergence zones in turbulent flows. In: Proceedings, 1988 Summer Program, Stanford NASA Centre for Turbulence Research. CTR-S88 1988;193-208.

Jensen JA. Field: A program for simulating ultrasound systems. Med Biol Eng Comput 1996;34:351-353.

Jensen JA, Svendsen NB. Calculation of pressure fields from arbitrarily shaped, apodized, and excited ultrasound transducers. IEEE Trans Ultrason Ferroelectr Freq Control 1992;39:262-267.

Jiamsripong P, Calleja AM, Alharthi MS, Dzsinich M, McMahon EM, Heys JJ, Milano M, Sengupta PP, Khandheria BK, Belohlavek M. Impact of acute moderate elevation in left ventricular afterload on diastolic transmitral flow efficiency: analysis by vortex formation time. J Am Soc Echocardiogr 2009;22:427-431.

Kasai C, Namekawa K, Koyano A, Omoto R. Real-time twodimensional blood flow imaging using an autocorrelation technique. IEEE Trans Son Ultrason 1985;32:458-464.

Kheradvar A, Assadi R, Falahatpisheh A, Sengupta PP. Assessment of transmitral vortex formation in patients with diastolic dysfunction. J Am Soc Echocardiogr 2012;25:220-227.

Kheradvar A, Pedrizzetti G. Vortex formation in the cardiovascular system. London: Springer-Verlag; 2012.

Kilner PJ, Yang GZ, Wilkes AJ, Mohiaddin RH, Firmin DN, Yacoub MH. Asymmetric redirection of flow through the heart. Nature 2000;404:759-761.

Kim HB, Hertzberg JR, Shandas R. Development and validation of echo PIV. Experiments Fluids 2004;36:455-462.

Lu J, Li W, Zhong Y, Luo A, Xie S, Yin L. Intuitive visualization and quantification of intraventricular convection in acute ischemic left ventricular failure during early diastole using color Doppler-based echocardiographic vector flow mapping. Int J Cardiovasc Imaging 2012;28:1035-1047.

Markl M, Kilner PJ, Ebbers T. Comprehensive 4-D velocity mapping of the heart and great vessels by cardiovascular magnetic resonance. J Cardiovasc Magn Reson 2011;13:7.

Mitchell DG. Color Doppler imaging: Principles, limitations, and artifacts. Radiology 1990;177:1-10.

Mohiaddin RH. Flow patterns in the dilated ischemic left ventricle studied by MR imaging with velocity vector mapping. J Magn Reson Imaging 1995;5:493-498.

Muth S, Dort S, Sebag IA, Blais MJ, Garcia D. Unsupervised dealiasing and denoising of color-Doppler data. Med Image Anal 2011;15: 577-588.

Nucifora G, Delgado V, Bertini M, Marsan NA, Van de Veire NR, Ng AC, Siebelink HM, Schalij MJ, Holman ER, Sengupta PP, Bax JJ. Left ventricular muscle and fluid mechanics in acute myocardial infarction. Am J Cardiol 2010;106:1404-1409.

Pedrizzetti G, Domenichini F. Nature optimizes the swirling flow in the human left ventricle. Phys Rev Lett 2005;95:108101.

Poh KK, Lee LC, Shen L, Chong E, Tan YL, Chai P, Yeo TC, Wood MJ. Left ventricular fluid dynamics in heart failure: Echocardiographic measurement and utilities of vortex formation time. Eur Heart J Cardiovasc Imaging 2012;13:385-393.

Prinz C, Faludi R, Walker A, Amzulescu M, Gao H, Uejima T, Fraser AG, Voigt JU. Can echocardiographic particle image velocimetry correctly detect motion patterns as they occur in blood inside heart chambers? A validation study using moving phantoms. Cardiovasc Ultrasound 2012;10:24.

Raffel M, Willert C, Wereley S, Kompenhans J. Post-processing of PIV data. In: Particle image velocimetry, (ed). A practical guide. Berlin/ New York: Springer-Verlag; 2007. p. 177-208.

Rodevand O, Bjornerheim R, Edvardsen T, Smiseth OA, Ihlen H. Diastolic flow pattern in the normal left ventricle. J Am Soc Echocardiogr 1999;12:500-507.

Sengupta PP, Pedrizzetti G, Kilner PJ, Kheradvar A, Ebbers T, Tonti G, Fraser AG, Narula J. Emerging trends in CV flow visualization. JACC Cardiovasc Imaging 2012;5:305-316.

Stewart KC, Charonko JC, Niebel CL, Little WC, Vlachos PP. Left ventricle filling vortex formation is unaffected by diastolic impairment. Am J Physiol Heart Circ Physiol 2012;303:H1255-H1262.

Tanné D, Bertrand E, Kadem L, Pibarot P, Rieu R. Assessment of left heart and pulmonary circulation flow dynamics by a new pulsed mock circulatory system. Experiments Fluids 2010;48:837-850. 
Thompson RB, McVeigh ER. Fast measurement of intracardiac pressure differences with 2-D breath-hold phase-contrast MRI. Magn Reson Med 2003;49:1056-1066.

Töger J, Kanski M, Carlsson M, Kovács SJ, Söderlind G, Arheden H, Heiberg E. Vortex ring formation in the left ventricle of the heart: Analysis by 4-D flow MRI and Lagrangian coherent structures. Ann Biomed Eng 2012;40:2652-2662.

Uejima T, Koike A, Sawada H, Aizawa T, Ohtsuki S, Tanaka M, Furukawa T, Fraser AG. A new echocardiographic method for identifying vortex flow in the left ventricle: numerical validation. Ultrasound Med Biol 2010;36:772-788.

Van Dantzig JM, Delemarre BJ, Bot H, Koster RW, Visser CA. Doppler left ventricular flow pattern versus conventional predictors of left ventricular thrombus after acute myocardial infarction. J Am Coll Cardiol 1995;25:1341-1346.
Wigstrom L, Ebbers T, Fyrenius A, Karlsson M, Engvall J, Wranne B, Bolger AF. Particle trace visualization of intracardiac flow using time-resolved 3-D phase contrast MRI. Magn Reson Med 1999; 41:793-799.

Yu A, Lovstakken L. Eigen-based clutter filter design for ultrasound color flow imaging: A review. IEEE Trans Ultrason Ferroelectr Freq Control 2010;57:1096-1111.

Yu AC, Cobbold RS. Single-ensemble-based eigen-processing methods for color flow imaging: Part I. The Hankel-SVD filter. IEEE Trans Ultrason Ferroelectr Freq Control 2008;55: 559-572.

Zhang H, Zhang J, Zhu X, Chen L, Liu L, Duan Y, Yu M, Zhou X, Zhu T, Zhu M, Li H. The left ventricular intracavitary vortex during the isovolumic contraction period as detected by vector flow mapping. Echocardiography 2012;29:579-587. 